\title{
Extending the Search Space for Novel Physical Forms of Pharmaceuticals and Biomolecules using High-Pressure Techniques
}

\author{
Dissertation \\ zur Erlangung des mathematisch-naturwissenschaftlichen Doktorgrades \\ "Doctor rerum naturalium" \\ der Georg-August-Universität Göttingen \\ im Promotionsprogramm Geowissenschaften / Geographie \\ der Georg-August University School of Science (GAUSS)
}

vorgelegt von

Sofiane Saouane

aus Annaba (Algerien)

Göttingen 2015 
Betreuungsausschuss:

Dr. Francesca P. A. Fabbiani,

Abteilung Kristallographie, Geowissenschaftliches Zentrum

Prof. Dr. Werner F. Kuhs,

Abteilung Kristallographie, Geowissenschaftliches Zentrum

Mitglieder der Prüfungskommission

Referent/in: $\quad$ Dr. Francesca P. A. Fabbiani

Korreferent/in: Prof. Dr. Werner F. Kuhs

weitere Mitglieder der Prüfungskommission:

Prof. Dr. Ralf Ficner

apl. Prof. Dr. Heidrun Sowa

Prof. Dr. Dietmar Stalke

Prof. Dr. Kai Tittmann

Tag der mündlichen Prüfung: 30.09.2015 


\section{Abstract}

This thesis, which is divided into two parts, describes the use of non-ambient crystallisation techniques, in particular high-pressure, to explore on one hand inclusion complex formation of cyclodextrins with drug molecules (part 1), and on the other hand the solid-state behaviour of imidazolium-based ionic liquids (part 2). Much of this thesis is devoted to establishing detailed crystallisation protocols for the compounds studied and to reporting full structural analysis of the resulting crystals, which have been investigated by single-crystal X-ray diffraction using both laboratory and synchrotron sources.

This work shows that the application of pressure has different effects on cyclodextrinbased inclusion complexes. When water is used as pressure transmitting medium, cyclodextrins undergo dissolution as function of increasing pressure, regardless of the inclusion state. Upon further pressurisation three events have been observed to take place: no crystallisation, crystallisation of host and guest molecules as separate entities or crystallisation of inclusion complexes. It has not been possible to rationalise the observed behaviour or predict which of the three events is most likely to occur for a given system. During the course of this work, novel inclusion complexes for $\alpha$ - and $\beta$-cyclodextrin have been obtained at both ambient- and highpressure conditions; full structural characterisation has enabled to identify two novel packing motifs.

As demonstrated for imidazolium-based ionic liquids, high-pressure is a powerful external factor for triggering crystal formation and phase transitions in this class of compounds. The solidstate behaviour of ionic liquids at high pressure has been correlated with the one at low temperature, proving that a thorough understanding and exploration of the crystallisation diagrams necessitates the use of both non-ambient techniques. The studies performed in this thesis demonstrate the importance of characterising crystalline phases by single-crystal X-ray diffraction methods. Thanks to the availability of accurate structural data it has been possible to unravel the elusive polymorphism of the most widely studied imidazolium-based ionic liquid, 1-butyl-3methylimidazolium hexafluorophosphate, which had been the subject of much debate and speculation in the literature. For 1-decyl-3-methylimidazolium chloride, structural data have enabled to distinguish between three distinct packing types of this ionic liquid in its hydrated forms, and to pin point subtle but important structural differences between the different crystalline phases. 


\section{Table of Contents}

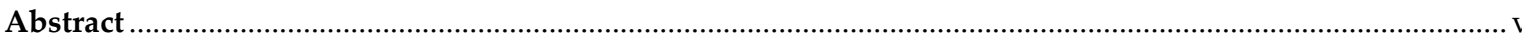

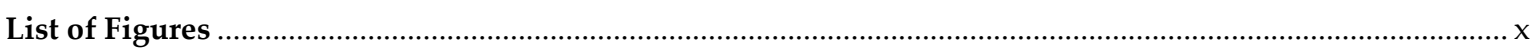

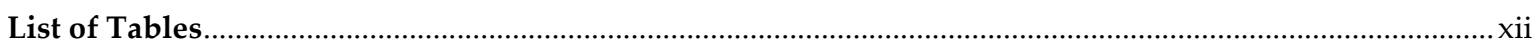

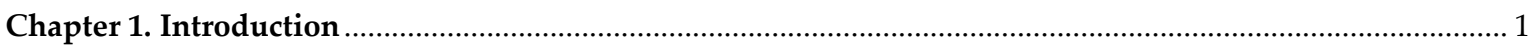

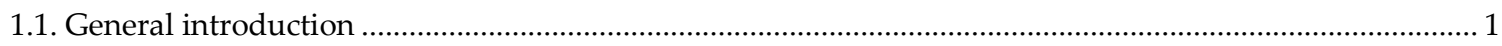

1.1.1. Polymorphism in the pharmaceutical industry ................................................................................... 2

1.1.2. Exploring drug polymorphism in pharmaceutical industry vs. academic research ............................ 3

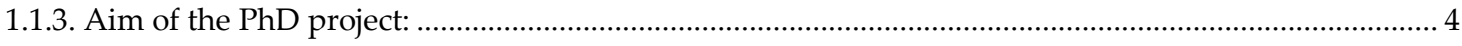

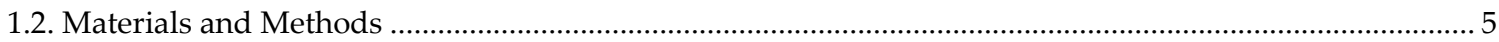

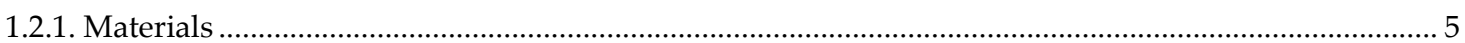

1.2.2. Methods

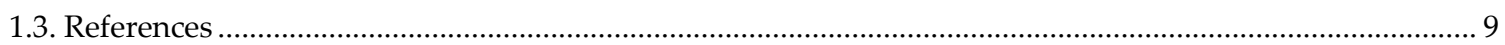

PART I. Exploring Inclusion Complex Formation of Cyclodextrins with Drug Molecules ........................... 13

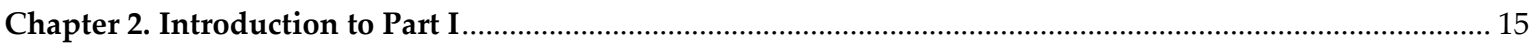

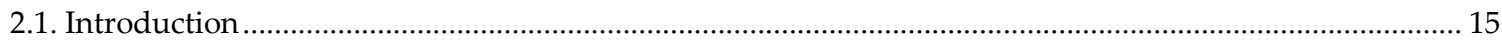

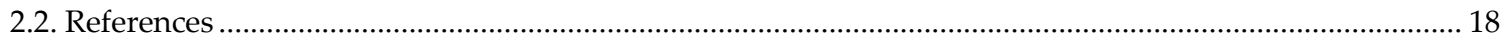

Chapter 3. $\alpha$-Cyclodextrin·succinic acid inclusion complex: a novel packing type of $\alpha$-cyclodextrin ........... 19

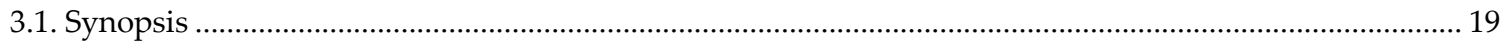

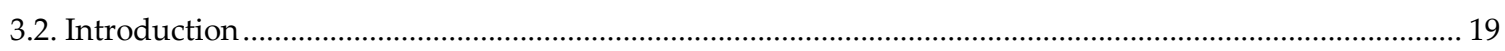

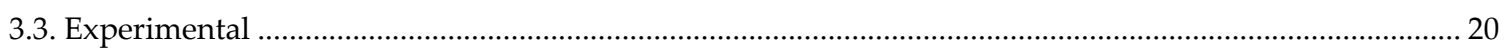

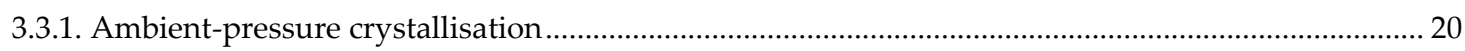

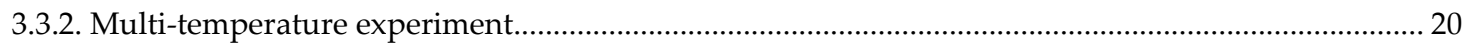

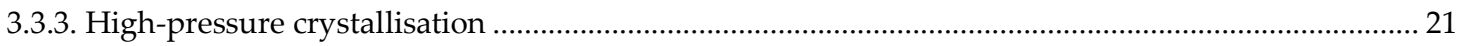

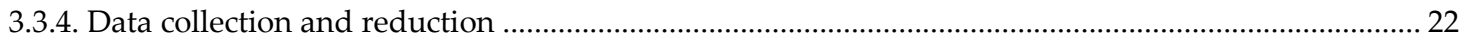

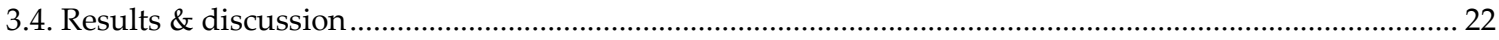

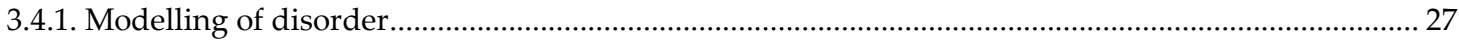

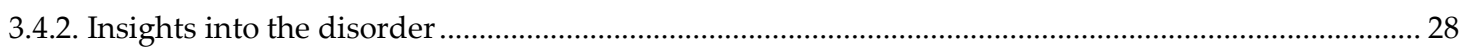

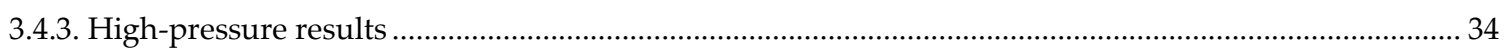

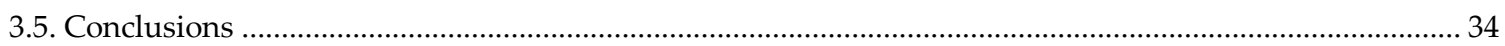

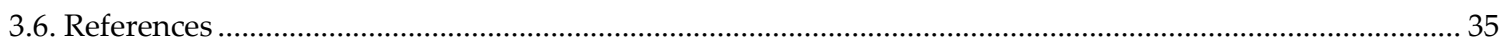

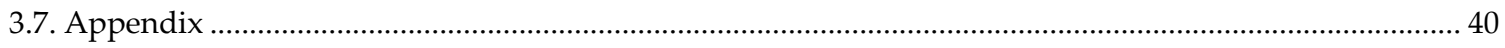

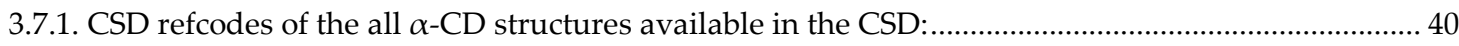

3.7.2. The evolution of the normalised unit cell parameters as function of temperature ........................... 40

3.7.3. Analysis of the anisotropic displacement parameters …................................................................. 42

Chapter 4. A new high-pressure form of $\beta$-cyclodextrin·paracetamol inclusion complex ............................. 45

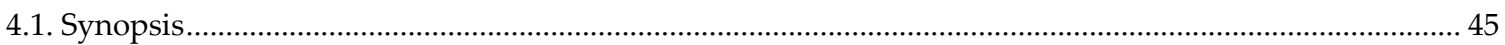




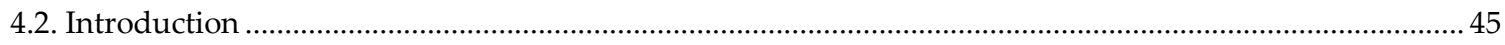

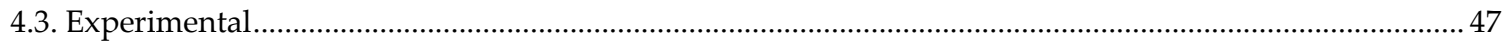

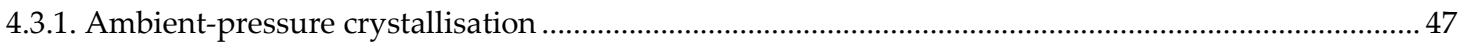

4.3.2. High-pressure in situ crystallisation of $\beta$-CD·paracetamol form $\mathrm{V}$.................................................... 47

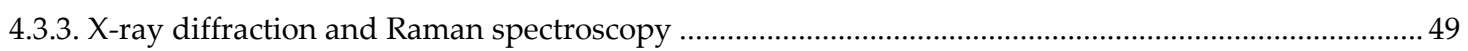

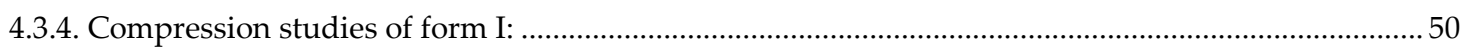

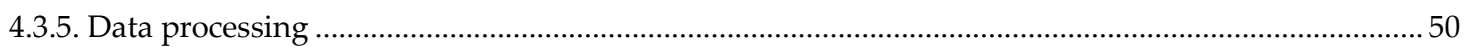

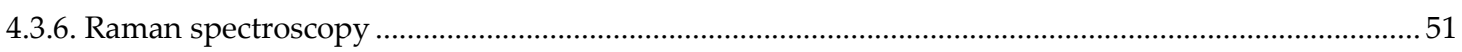

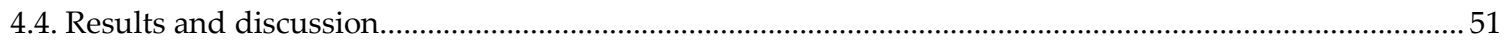

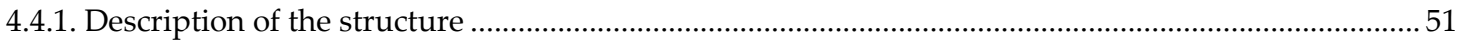

4.4.2. Crystal packing similarities between $C 2221$ and $P 21$ structures .......................................................54

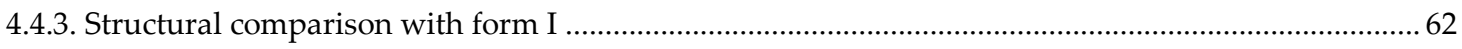

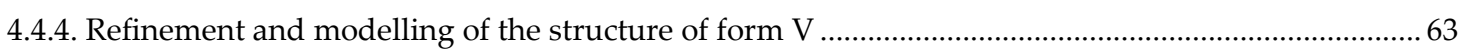

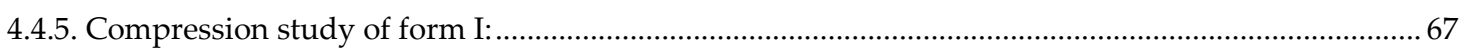

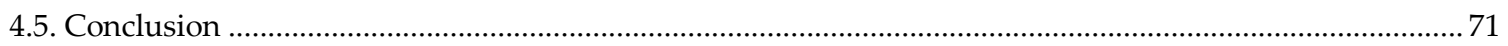

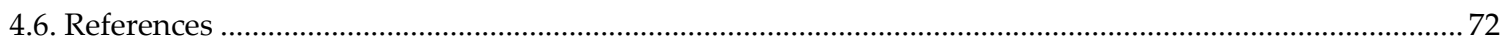

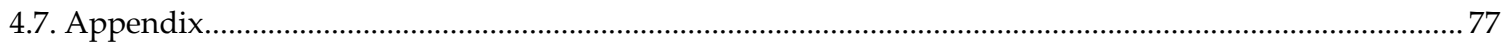

Chapter 5. Screening of inclusion complex formation at ambient- and high-pressure conditions ................. 81

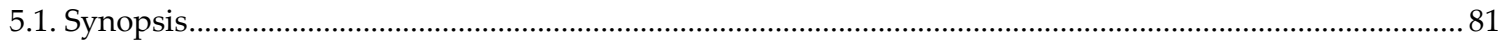

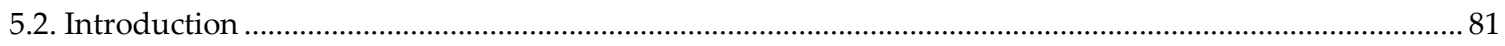

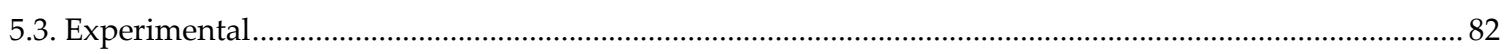

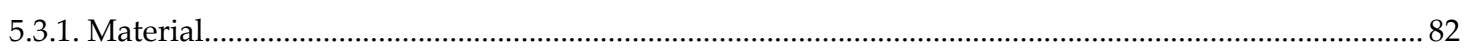

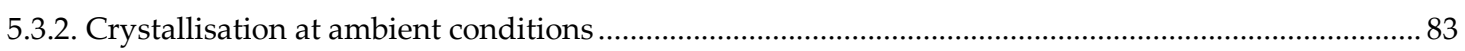

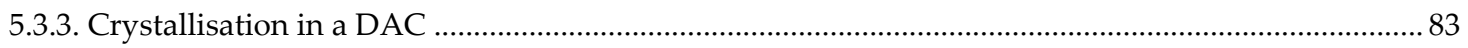

5.3.4. High-pressure experiment using a stationary gas compressor ........................................................... 87

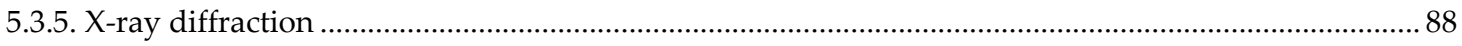

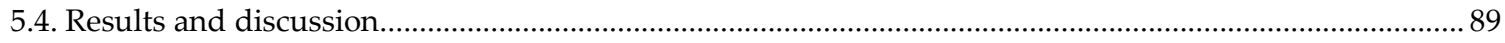

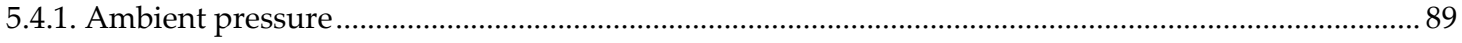

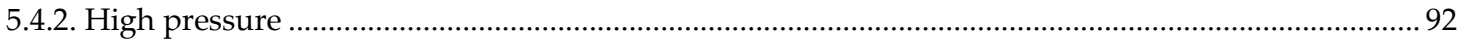

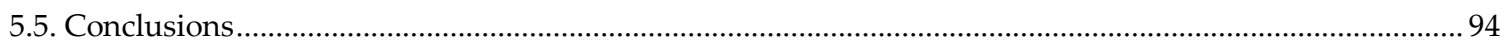

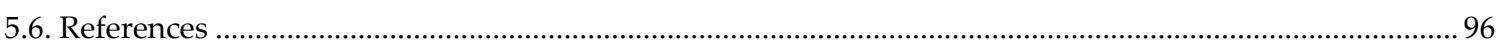

PART II. Exploring the Solid-State Behaviour of Imidazolium-Based Ionic Liquids ...................................99

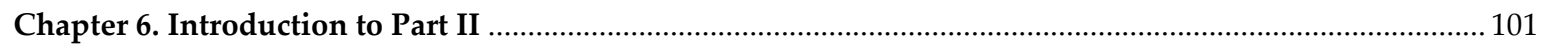

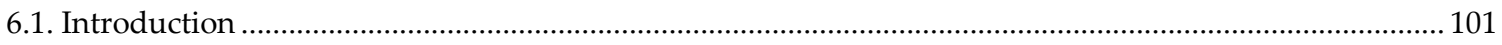

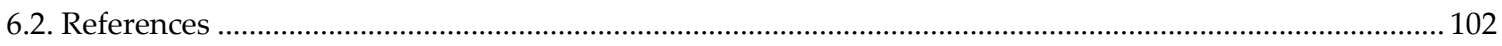

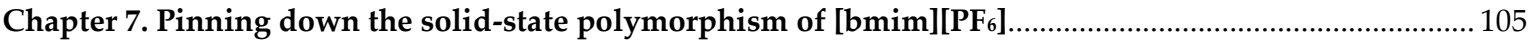

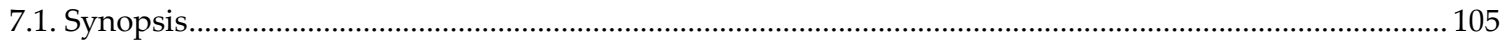

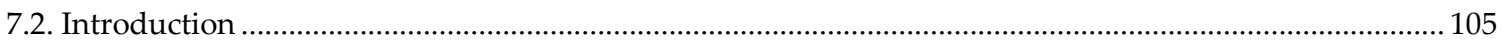




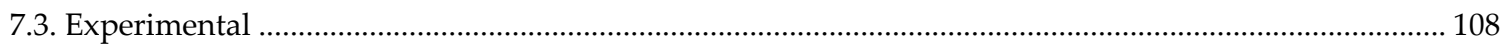

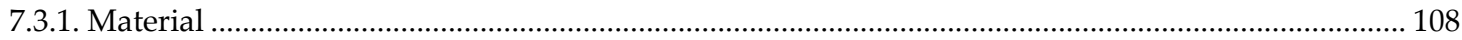

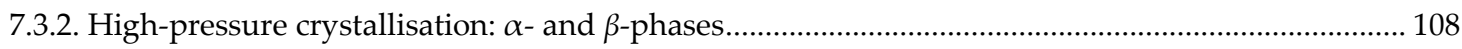

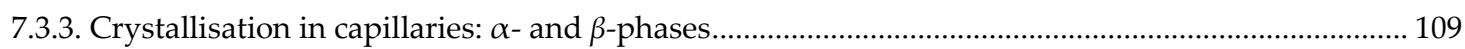

7.3.4. Crystallisation using a Linkam stage: $\alpha$ - $\beta$ - and $\gamma$-phases ............................................................ 109

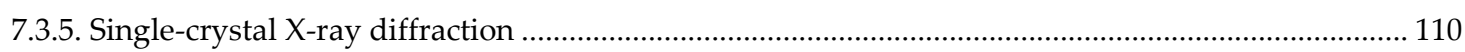

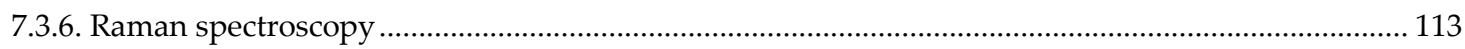

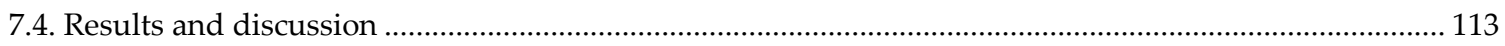

7.4.1. Conformation of the $[\mathrm{bmim}]^{+}$cation in the solid state ................................................................. 113

7.4.2. Disorder of the $[\mathrm{PF} 6]^{-}$anion in the solid state ............................................................................ 116

7.4.3. Crystal structures of the three polymorphs ...................................................................................... 117

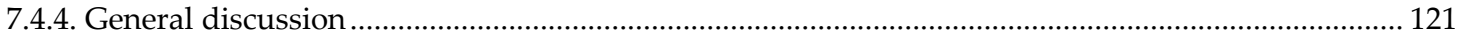

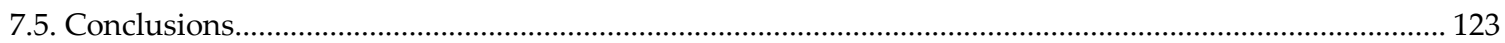

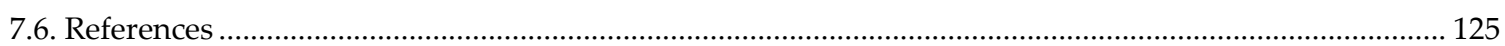

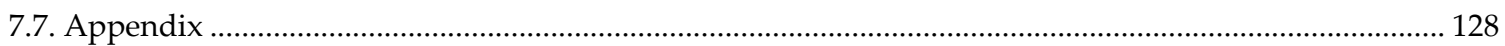

Chapter 8. Structural behaviour of long-chain imidazolium-based ionic liquid [C10mim]Cl-water

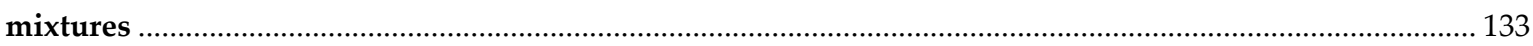

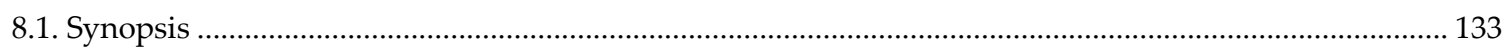

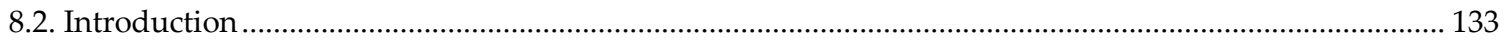

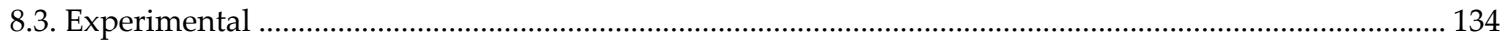

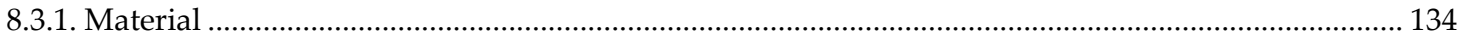

8.3.2. Low-temperature crystallisation technique ................................................................................... 134

8.3.3. High-pressure crystallisation techniques..................................................................................... 136

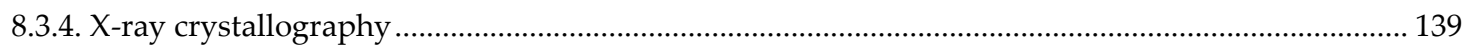

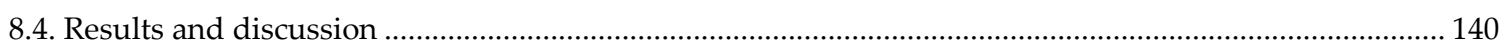

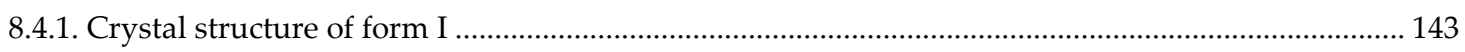

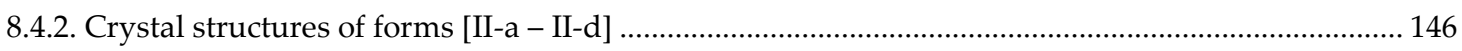

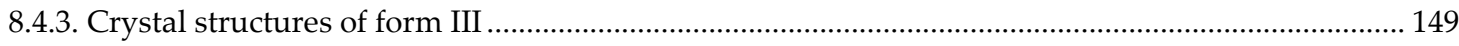

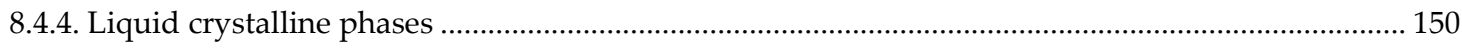

8.4.5. [ $\left.\mathrm{C}_{10 \mathrm{mim}}\right] \mathrm{Cl}$ as a crystallisation solvent for active pharmaceutical ingredients ............................... 151

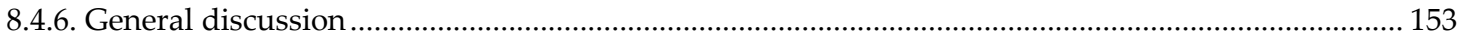

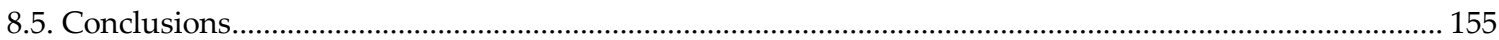

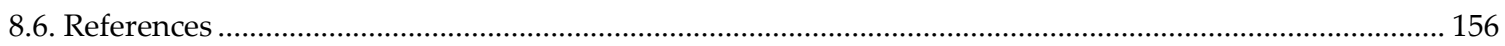

8.7. Appendix: Qualitative analysis of $[\mathrm{C} 10 \mathrm{mim}] \mathrm{Cl}$ liquid crystal formation ............................................... 160

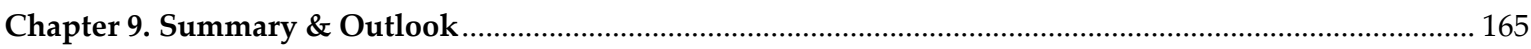

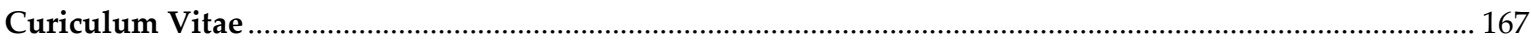

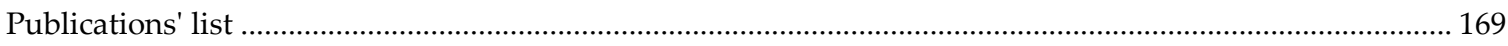




\section{List of Figures}

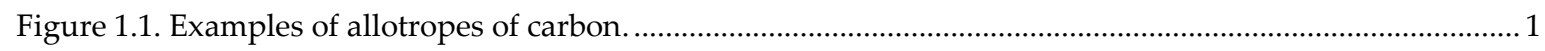

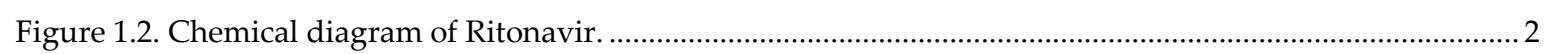

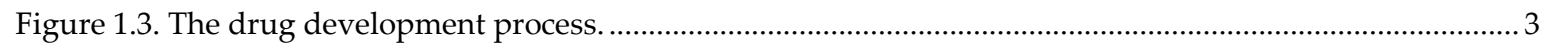

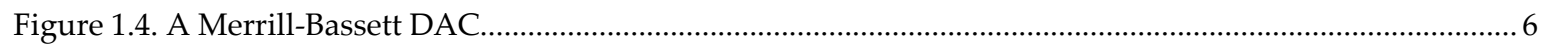

Figure 1.5. Cartoon summarising the Raman spectroscopy technique. ............................................................... 8

Figure 2.1. Chemical diagram of $\alpha-\mathrm{D}$-glucopyranose unit and the general shape of a CD molecule ................. 15

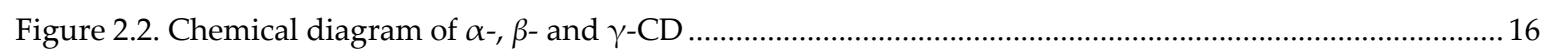

Figure 3.1. Diagrams and numbering schemes of succinic acid, $\alpha$-CD and $\alpha$-D-glucopyranose........................20

Figure 3.2. High-pressure crystallisation attempts of $\alpha$-CD·SA inclusion complex ...........................................2 21

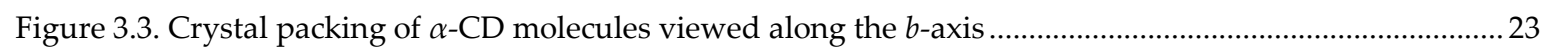

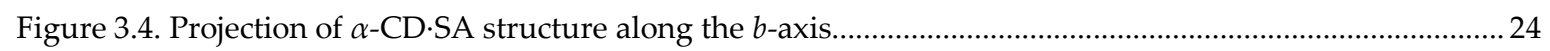

Figure 3.5. The honeycomb packing mode of $\alpha$-CD·SA structure along the $c$-axis............................................2 25

Figure 3.6. H-bonded motif formed by SA molecules in the asymmetric unit viewed along the $a$ - and b-axes. 26

Figure 3.7. Fobs-Fcalc maps in green showing the electron density before and after modelling SA inside $\alpha$-CD

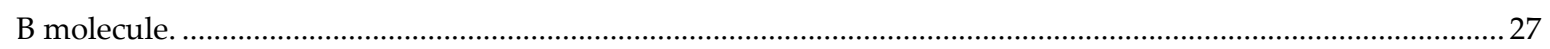

Figure 3.8. The evolution of the normalised unit cell parameters as function of temperature ...........................29

Figure 3.9. Evolution of the normalised distances between dimers in $\alpha$-CD·SA............................................... 30

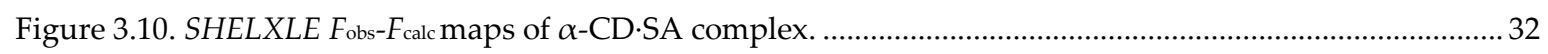

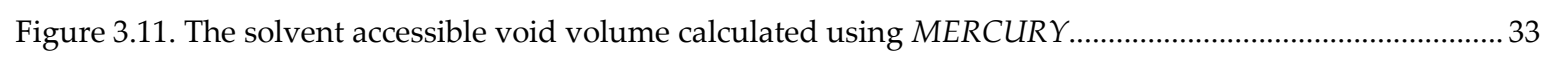

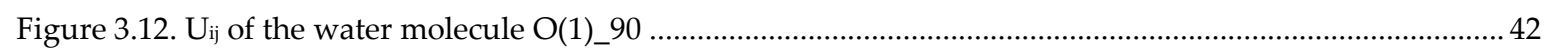

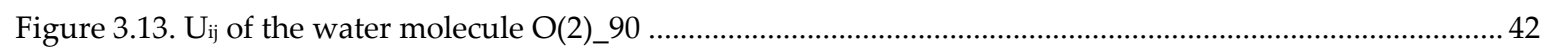

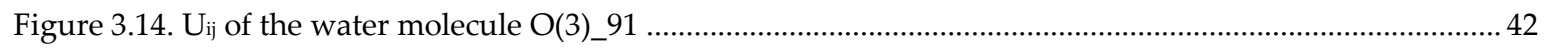

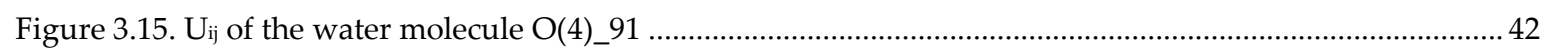

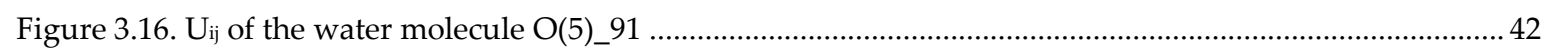

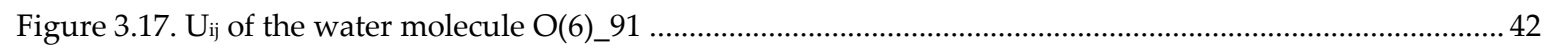

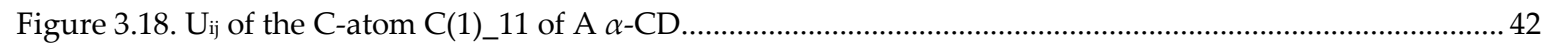

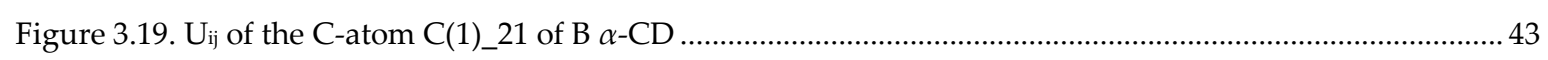

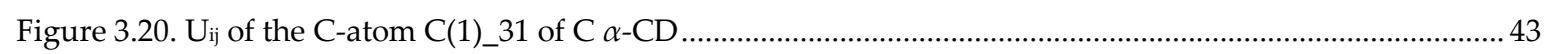

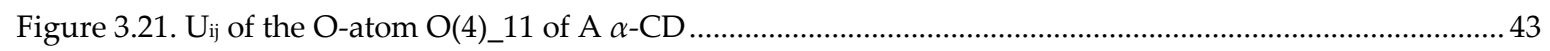

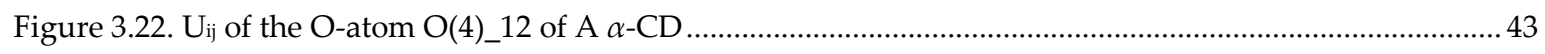

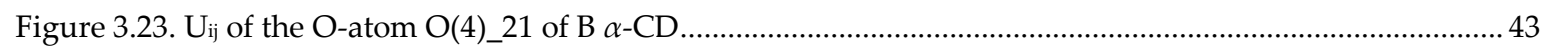

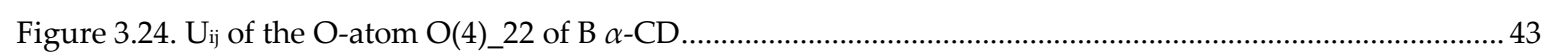

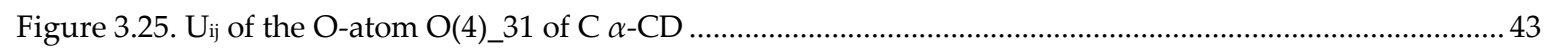

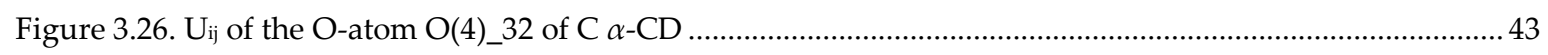

Figure 3.27. Overlay of $\alpha$-CD·SA structures at $180 \mathrm{~K}$ and $270 \mathrm{~K}$ viewed along the $b$-axis................................ 44

Figure 4.1. Diagrams and numbering schemes of $\beta$-CD, $\alpha$-D-glucopyranose and paracetamol...........................46

Figure 4.2. Crystallisations procedures of $\beta$-CD·paracetamol inclusion complexes ............................................4 47

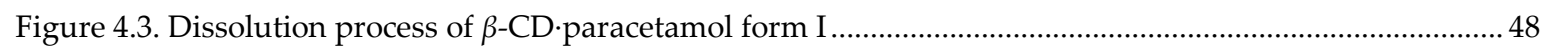


Figure 4.4. Crystallisation of $\beta$-CD paracetamol form $\mathrm{V}$ from saturated solution .............................................. 48

Figure 4.5. Crystals of $\beta$-CD·paracetamol form V crystallised at $0.8 \mathrm{GPa}$....................................................... 48

Figure 4.6. Compression study of $\beta$-CD·paracetamol form I using FC43 and THF as PTM.. ............................50

Figure 4.7. Summary and examples of $\beta$-CD packing types in the literature.....................................................53

Figure 4.8. Packing feature of $\beta$-CD·paracetamol form $\mathrm{V}$ inclusion complex. .................................................. 54

Figure 4.9. Relationship between monoclinic and orthorhombic unit cells ...................................................... 55

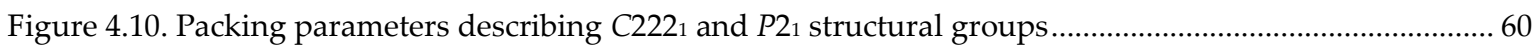

Figure 4.11. Comparison between $C 2221, P 21$ and $\beta$-CD.paracetamol form $\mathrm{V}$ packing types .............................. 61

Figure 4.12. H bonds formed by paracetamol and water molecules inside $\beta$-CD cavity .................................... 63

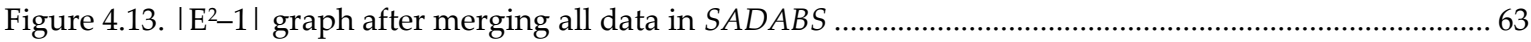

Figure 4.14. Raman spectra of $\beta$-CD hydrate, paracetamol form II, $\beta$-CD-paracetamol form I and the three crystals obtained at high-pressure in a DAC.

Figure 4.15. Structural model and electron density maps of paracetamol and water molecules generated with shelXle....... 66

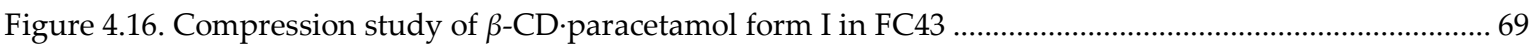

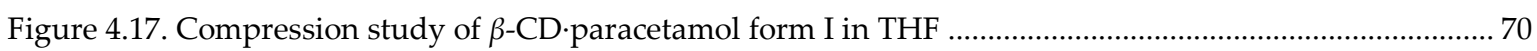

Figure 4.18. Raman spectrum of the solution surrounding the crystal of $\beta$-CD.paracetamol form V ……......... 79

Figure 5.1. Chemical diagrams of the investigated compounds ....................................................................... 82

Figure 5.2. Crystallisation attempt of a hot aqueous solution of $\alpha-\mathrm{CD} \cdot 5 \mathrm{FU}$ in a DAC .................................... 85

Figure 5.3. Crystallisation attempt of a hot aqueous solution of $\beta$-CD·5FU in a DAC........................................ 85

Figure 5.4. High-pressure crystallisation attempt of an equimolar solution of $\beta$-CD and L-ascorbic acid ......... 86

Figure 5.5. Recrystallisation attempts of $\beta$-CD.SA complex at high pressure .................................................... 86

Figure 5.6. Exploring the behaviour of single crystals of amoxicillin and $\beta$-CD at high-pressure ..................... 87

Figure 5.7. Investigating the dissolution at high pressure of amoxicillin in water............................................. 87

Figure 5.8. $\beta$-CD and amoxicillin solution $(\mathrm{a})$ before and $(\mathrm{b})$ after applying $0.5 \mathrm{GPa}$ pressure ..........................8 88

Figure 5.9. Slow evaporation of the filtrate of $\beta$-CD·amoxicillin solution ........................................................... 88

Figure 7.1. Chemical diagram of $[\mathrm{bmim}]\left[\mathrm{PF}_{6}\right]$ with atom numbering scheme................................................ 106

Figure 7.2. Stages of isothermal in situ crystal growth of the $\beta$-phase of [bmim][PF6] in the DAC at $293 \mathrm{~K} . . . .109$

Figure 7.3. Optical images of crystals of [bmim] [PF6] grown on a Linkam stage ........................................... 110

Figure 7.4. Dihedral angles for $\mathrm{N} 1-\mathrm{C} 7-\mathrm{C} 8-\mathrm{C} 9$ and $\mathrm{C} 7-\mathrm{C} 8-\mathrm{C} 9-\mathrm{C} 10$ for defining the [bmim]+ cation conformation

Figure 7.5. Raman spectra of $[\mathrm{bmim}]\left[\mathrm{PF}_{6}\right]$ polymorphs collected at different conditions of temperature (top) and pressure (bottom)

Figure 7.6. Distribution of the conformers of [bmim $]^{+}$-containing structures found in the CSD 116

Figure 7.7. Dimeric arrangements in the three polymorphs of $[\mathrm{bmim}]\left[\mathrm{PF}_{6}\right]$ viewed along two different stacking directions

Figure 7.8. Crystal packing of $\left[\mathrm{bmim}^{-}\left[\mathrm{PF}_{6}\right], \alpha\right.$-form and of 1-n-dodecyl-3-methylimidazolium hexafluorophosphate, CSD Ref. code HIWNOQ. 118

Figure 7.9. Crystal packing in the three polymorphs of $[\mathrm{bmim}]\left[\mathrm{PF}_{6}\right]$. 119 
Figure 7.10. Powder patterns simulated using the experimentally-determined single-crystal structures as input... 132

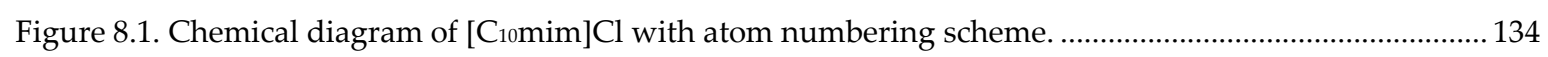

Figure 8.2. Optical microscopy images of the low-temperature crystal growth of forms I and II-a .................. 135

Figure 8.3. Optical microscopy images of the high-pressure growth of form I in a DAC ................................ 136

Figure 8.4. Optical microscopy images of the high-pressure growth of form II-c in a DAC .............................. 137

Figure 8.5. Optical microscopy images of the high-pressure growth of form II-d in a DAC............................. 137

Figure 8.6. Optical microscopy images of the high-pressure growth of form III at $1.14 \mathrm{GPa}$........................... 138

Figure 8.7. Optical microscopy images of the high-pressure growth of form III at $0.60 \mathrm{GPa} \ldots \ldots \ldots \ldots \ldots \ldots \ldots \ldots \ldots . . . . . . . . . . . .138$

Figure 8.8. Crystal packing of $\left[\mathrm{C}_{10 \mathrm{mim}}\right] \mathrm{Cl}$ form I seen along the $b$-axis........................................................... 143

Figure 8.9. [C $\left.\mathrm{C}_{10 \mathrm{mim}}\right] \mathrm{Cl}$ cluster and its corresponding Hirshfeld surfaces mapped with the $d_{\text {norm }}$ property ...... 145

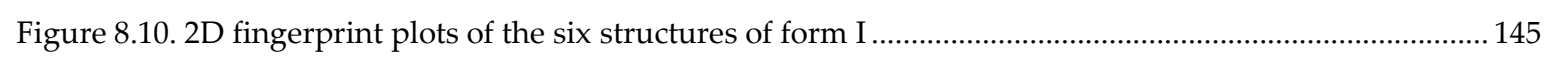

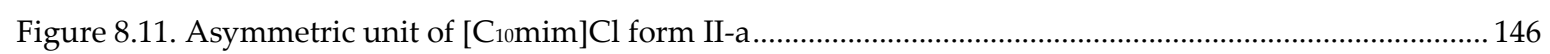

Figure 8.12. Crystal packing of $\left[\mathrm{C}_{10} \mathrm{mim}\right] \mathrm{Cl}$ form II-a seen along the $b$-axis ................................................... 146

Figure 8.13. Comparison between the curving of the alkyl chains of Res2 in all form II structures reported in this work.

Figure 8.14. Comparison between the curving of the alkyl chains of Res2 in: form II-a, [C14mim]Cl and

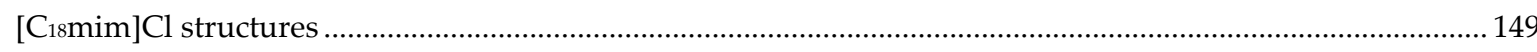

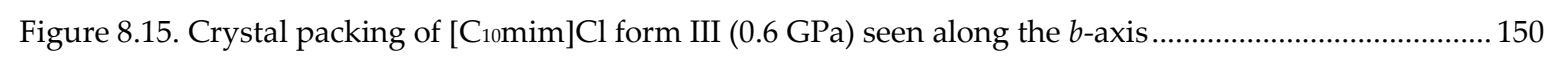

Figure 8.16. High-pressure crystallisation attempt of paracetamol in [C10mim] Cl solution in a DAC .............. 152

Figure 8.17. High-pressure crystallisation attempt of paracetamol in [C10mim] Cl solution in a DAC .............. 152

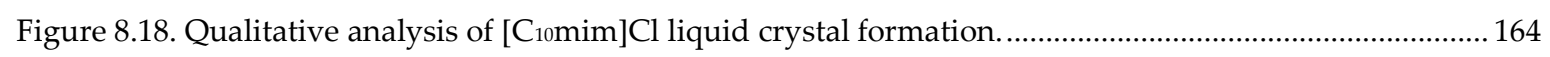

\section{List of Tables}

Table 3.1: Crystallographic data of the multi-temperature structures of $\alpha$-CD·SA inclusion complex ...............31

Table 3.2: evolution of the normalised unit cell parameters as function of temperature....................................... 41

Table 4.1. Crystallographic data of the five forms of $\beta$-CD·paracetamol inclusion complexes .............................52

Table 4.2. $\beta$-CD inclusion complex structures reported in the CSD and similar to $\beta$-CD-paracetamol form V. . 58

Table 4.3. RMSD values $(\AA)$ and maximum number of fitted molecules up to a maximum number of 30 molecules in a cluster, calculated using Crystal Packing Similarities in MERCURY...........................................59

Table 4.4. Crystallographic data of the compression study of $\beta$-CD·paracetamol form I inclusion complex ..... 68

Table 4.5. RMSD values $(\AA)$ per $\beta$-CD dimer calculated using Crystal Packing Similarities in MERCURY ..........77

Table 4.6. PXRD similarities of all $C 2221$ and $P 21$ structures studied in this chapter calculated using Crystal

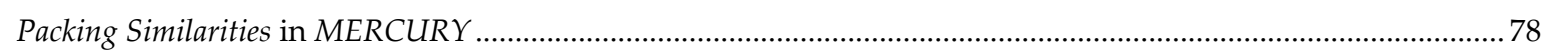

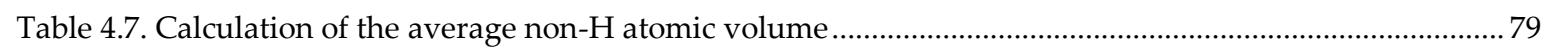

Table 4.8. Effect of Pressure and temperature on the average atomic volume..................................................... 80

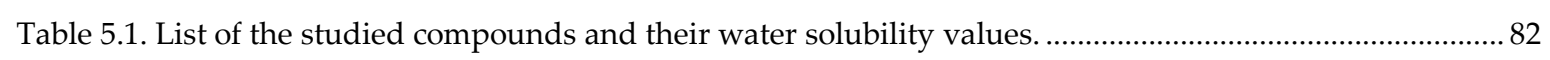

Table 5.2. Summary of the experimental inclusion complex screening at ambient-pressure condition............... 83

Table 5.3. Summary of the experimental inclusion complex screening at high-pressure condition. ....................84 
Table 5.4. Summary of the single-crystal X-ray diffraction results on the inclusion complex screening investigated at ambient-pressure conditions.

Table 5.5. Crystal data of all measured single crystals following the inclusion complex screening at ambient conditions.

Table 5.6. Summary of the single-crystal X-ray diffraction results on the inclusion complex screening investigated at high- pressure conditions.

Table 7.1. Summary of crystallisation conditions and observed polymorphs of $[\mathrm{bmim}]\left[\mathrm{PF}_{6}\right]$ reported in the literature.

Table 7.2. Crystallographic data for the three polymorphs of [bmim] $\left[\mathrm{PF}_{6}\right]$ discussed in this chapter. 112

Table 7.3. Torsion angles and cation conformation for the polymorphs of $\left[\mathrm{bmim}^{2}\left[\mathrm{PF}_{6}\right]\right.$ at different experimental conditions.

Table 7.4. Geometric parameters describing the planar or nearly planar centrosymmetric and pseudocentrosymmetric dimeric arrangements of the cations in the three polymorphs of [bmim] $\left.\mathrm{PF}_{6}\right]$.

Table 7.5. Coordination numbers for the cation and anion, as well as short $\mathrm{H} \cdots \mathrm{F}$ contacts in the three polymorphs of $[\mathrm{bmim}]\left[\mathrm{PF}_{6}\right]$

Table 7.6. Short contacts with $\mathrm{H}$...F distances $<$ sum of $\mathrm{vdW}$ radii. $\mathrm{vdW}$ radius for $\mathrm{H}=1.20 \AA$, $\mathrm{vdW}$ radius for $\mathrm{F}=1.47 \AA$ A. The programs MERCURY ${ }^{28}$ and PLATON ${ }^{45}$ were used to check these values. 130

Table 8.1. Crystallographic data for [C10mim] $\mathrm{Cl}$ structures discussed in this chapter 141

Table 8.2. Summary of the crystallisation conditions of the [Cnmim]X structures ( $\mathrm{C} \geq 8, \mathrm{X} \equiv$ halide anion) reported in the literature and in this work

Table 8.3. Summary of the dissimilarity index and supramolecular construct types obtained using XPac...... 144

Table 8.4. Summary of the differences in the site occupancies of all form II structures presented in this work

Table 8.5. Summary of dihedral angles of the decyl side chain of $\left[\mathrm{C}_{10 \mathrm{mim}}\right]^{+}$cation and the reference angle $\mathrm{C}(2)-\mathrm{N}(1)-\mathrm{C}(7)-\mathrm{C}(8)$ seen in the low-temperature structures of form I and II-a...... 148 


\section{Acknowledgements}

Various people and institutions shaped my academic and personal development, and supported the successful completion of this work. I appreciated the opportunity to work in a lively and challenging environment in the department of crystallography at the University of Göttingen.

Firstly, I am indebted to my supervisor Dr. Francesca Fabbiani, without whom this work would have never happened. I am grateful for her teaching, support, caring, trust and patience. I have developed professionally and personally benefiting from her knowledge and experience.

I am grateful to Prof. Werner Kuhs for his constant support, his critical feedback and mentoring. It has been a pleasure to share lunch-time discussions beyond science. I am especially thankful to him for encouraging, trusting and supporting me to chair our department's seminars.

My acknowledgements go to the Deutsche Forschungsgemeinschaft (DFG), Deutsches Elektronen-Synchrotron (DESY) and several other institutions for sponsoring my PhD project. I also thank our collaborators for their help and trust. I would also like to acknowledge my thesis committee for taking time to read and evaluate my work.

Rubén Granero-García endured my endless theoretical questions; I was very lucky to have him as an officemate throughout the years of my PhD. Special thanks to Ulf Kahmann for all his technical support, his friendliness and the daily Franzbrötchen on my office desk. I thank Dr. Andrzej Falenty for his camaraderie. Many thanks to all my other colleagues who supported me along the way. Special thanks to Katharina Kamphausen for supporting me and proof-reading my thesis, and not to forget little Ada Dittrich-Fabbiani, who taught me how to take breaks from work and enhanced my babysitting skills.

Finally, I thank my family for their endless love and support throughout the course of my studies even from far away. 


\section{Chapter 1. Introduction}

\subsection{General introduction}

In this thesis, the term "physical form" defines any form in which an organic molecule can exist in the solid state. This definition encompasses crystalline as well as amorphous materials, although only crystalline materials are the subject focus of this thesis. If upon crystallisation the resulting crystal structure contains only the compound of interest, i.e. a single component crystal is formed, the physical form is commonly termed a crystalline polymorph of that particular compound. If the solid form is composed of distinct chemical species, the solid phase is referred to as a multicomponent crystal. Hydrates, solvates, salts, co-crystals and inclusion complexes are all examples of multicomponent crystals.

In text books, polymorphism is often described through the allotropy of carbon; the term allotropy is more pertinent here since carbon is an element. Carbon exists in several different natural and synthetic solid forms, namely diamond, lonsdaleite, graphite, graphene, carbynes, fullerenes and nanotubes (Figure 1.1). ${ }^{1}$
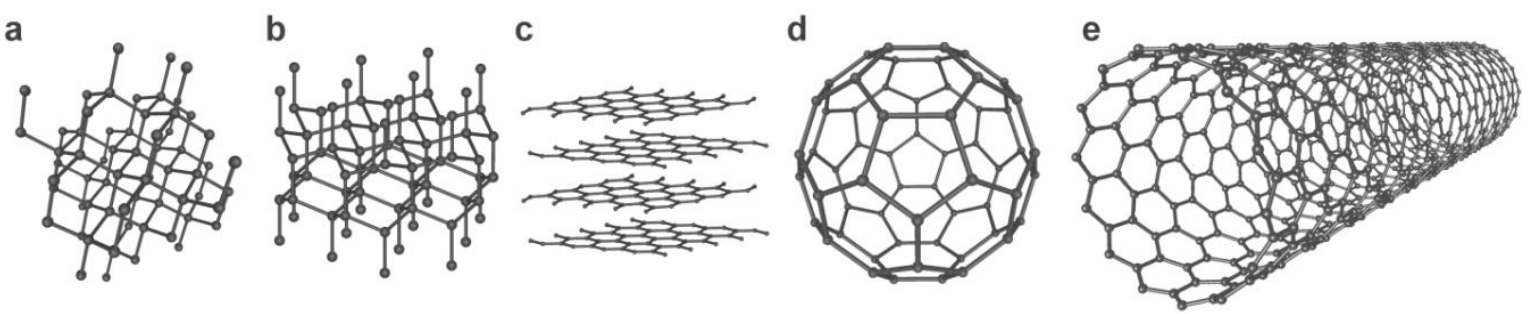

Figure 1.1. Examples of allotropes of carbon. a) diamond (cubic); b) lonsdaleite (hexagonal); c) graphite; d) C60 fullerene; e) single walled carbon nanotube. ${ }^{2}$

One of the earliest und undisputed definitions of polymorphism introduced by Haleblian and McCrone in a seminal paper about the subject was: "a polymorph is a solid crystalline phase of a given compound resulting from the possibility of at least two different arrangements of the molecules of that compound in the solid state". ${ }^{3}$ In simpler words: "Polymorphism is the phenomenon in which the same chemical compound exhibits different crystal structures". ${ }^{4}$ According to an extension of these definitions, in principle multicomponent crystals can also exist in different polymorphic forms.

Because in theory any compound can show polymorphism and different polymorphs have different physical and chemical properties, polymorphism is an area of intense research not only at the academic level but also within an industrial context. In industry, it plays a particularly prominent role in materials science (e.g. for dyes, agrochemicals, pigments, energetic materials, 
ceramic and metal engineering), food science (e.g. for chocolate or margarine) and pharmaceutical sciences. ${ }^{5}$

\subsubsection{Polymorphism in the pharmaceutical industry}

The considerable attention and importance of drug polymorphism in the pharmaceutical industry was triggered in the 1990s by the accounts of Abbot Laboratories in the now well-known "Ritonavir story". $6-8$ Abbots Laboratories had reported and patented only one crystal form of Ritonavir by the end of 1993. In mid-1998 and after only 18 months of marketing, several batches of Ritonavir (Figure 1.2), a protease inhibitor used to treat human immunodeficiency virus (HIV) infection and commercialised by the same company under the trade name Norvir ${ }^{\circledR}, 6$ failed the dissolution testing. After investigating the dissolution failure, the pharmaceutical company discovered that the active ingredient underwent a phase transition within the gel capsule to a thermodynamically more stable form, termed form II. The poor solubility of this form II (less than $50 \%$ than form I) caused a low bioavailability of Ritonavir. The pharmaceutical company was imposed to temporarily withdraw the drug from the market to investigate a reformulation procedure. Eventually, a new formulation was developed, approved and marketed in 1999, though this process proved very costly (ca. 250 million USD). ${ }^{8}$

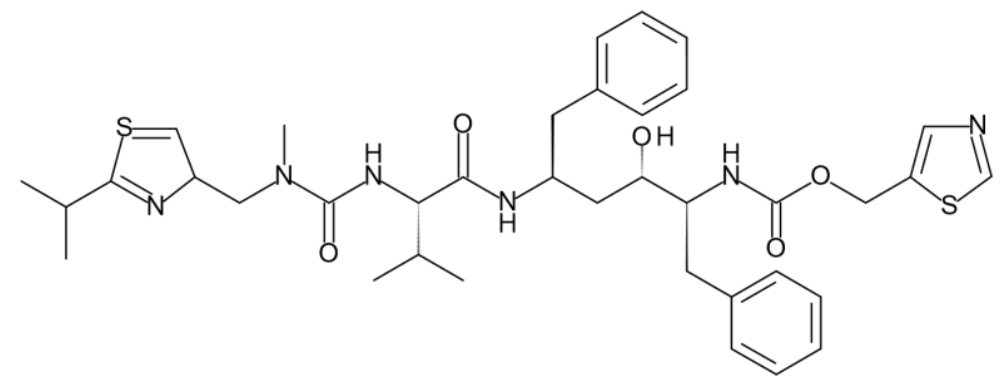

Figure 1.2. Chemical diagram of Ritonavir.

By the end of the 1990s, the Ritonavir story generated an extremely heated discussion and was the trigger of significant reconsiderations by pharmaceutical industries concerning the polymorphic behaviour of active pharmaceutical ingredients in and even before all development stages. This example had repercussions on the perception of polymorphism among pharmaceutical companies and led to the implementation of stricter industrial polices for its detection and control, which are also enforced by health agencies, such as the Food and Drug Administration (FDA) in the United States of America, the European Medicines Agency within the European Union (EMA), and at the international level by the International Conference on Harmonisation (ICH).

Conversely, beneficial opportunities for both the company and the patient can be created by a thorough understanding of solid-state properties of drug. The differences in the properties 
between two polymorphs are due to structural differences, which are governed by intermolecular interactions and possibly by different molecular conformations. The properties affected by drug polymorphism are numerous; they include lattice energies, density, melting points, solubility, dissolution rate, bioavailability, stability and processability. Hence, understanding the solid-state behaviour of drugs is of great interest to pharmaceutical companies in order to achieve the best efficiency in drug manufacturing. In addition, polymorphism of drugs enables great and lucrative opportunities for securing and controlling intellectual property.., 9

\subsubsection{Exploring drug polymorphism in pharmaceutical industry vs. academic research}

The prevalence of drug polymorphism has been estimated from 32 to $51 \%$ within the family of small drug molecules, ${ }^{10}$ and to $85 \%$ if solvates and hydrates are included. ${ }^{11}$ Nowadays, thorough exploration of the polymorphic behaviour of drug substances is an indispensable step in drug development, as shown schematically in Figure 1.3.

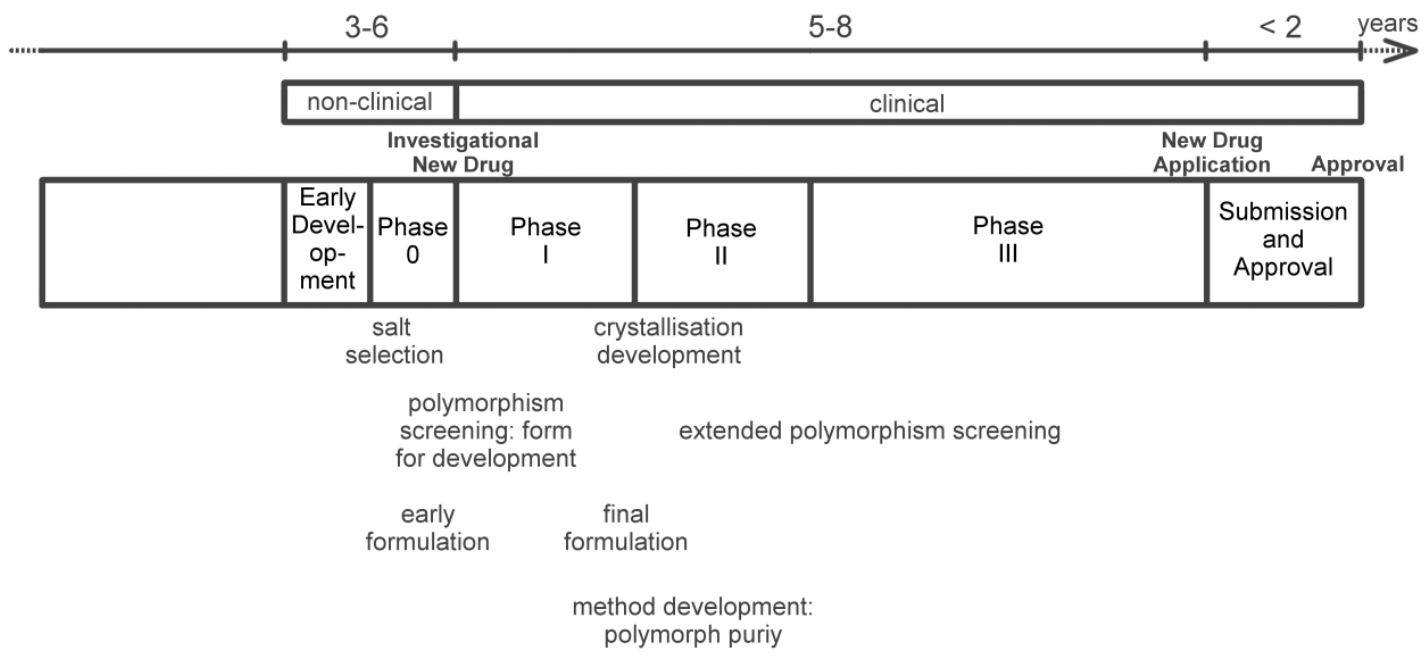

Figure 1.3. The drug development process. Figure modified from page 9 of reference 9.

The conventional methods used by pharmaceutical companies for screening the polymorphic behaviour of drug candidates are solvent- and temperature based. In a very recent paper, Lee summarised and referenced more than 15 different methods of polymorphic screening, ${ }^{12}$ such as: solvent selection (single or binary mixtures), thermal methods, mechanical activation, exposure to humidity and organic vapours, and other exotic techniques such as laser-induced crystallisation and addition of tailor-made additives. Within this article, references are made to mechanical activation processes ${ }^{13,14}$ involving applying mechanical stress in the form of grinding and compression, ${ }^{13}$ or applying pressure and shear forces. ${ }^{14}$ These processes can induce solid-tosolid phase transitions. Braga et al. ${ }^{14}$ further introduced "less conventional techniques" to investigate drug polymorphism, among which high-pressure crystallisation is reported. 
The pressure variable in polymorphism investigations is still rarely used in the pharmaceutical industry. High-pressure research on organic molecules is a relatively young research field, which has proven to be particularly fruitful with respect to producing new polymorphs. ${ }^{15-17}$ High-pressure research using diamond-anvil cells (DAC) is a well-established method in Physics and Geoscience; it has only recently been branched and introduced to organic molecules, as described by Boldyreva. ${ }^{18}$ In the past two decades, the use of high-pressure techniques has been enormously simplified both in terms of sample preparation and of data collection/processing strategies, thanks to progress in the design of new DACs, ${ }^{19-22}$ the high brilliance of synchrotron light sources, as well as progress in laboratory $\mathrm{X}$-ray sources, detectors and easy-to-use software. The wide range of compounds now routinely amenable to such studies is exemplified in three recent special issues published in Acta Crystallographica, ${ }^{23}$ Zeitschrift für Kristallographie ${ }^{24}$ and High-Pressure Research and a dedicated article in IUCrJ. ${ }^{25}$ These technological achievements have extended the range of compounds amenable for high-pressure studies. Indeed, X-ray diffraction on a single-crystal in a DAC can now be performed routinely in the laboratory.

\subsubsection{Aim of the PhD project:}

In his book on "Polymorphism in Molecular Crystals", Bernstein quotes a speech delivered by Eugene Sun (Abbott laboratories) during a press conference in 1998 about Norvir®, saying: "There are many mysteries of nature that we have not yet solved. Hurricanes, for example continue to occur and often cause massive devastation. Meteorologists cannot predict months in advance when and with what velocity a hurricane will strike a specific community. Polymorphism is a parallel phenomenon. We know that it will probably happen, but not why or when. Unfortunately, there is nothing we can do today to prevent a hurricane from striking any community or polymorphism from striking any drug." 5

While there is some level of truth in this statement, the polymorphic behaviour of a drug substance can be handled best if different methods are used to explore its solid-state behaviour.

This work focuses on the use of non-ambient techniques, especially high pressure, to investigate physical forms of drug molecules, cyclodextrins (CDs) and ionic liquids (ILs), comparing and contrasting the results with the behaviour observed at ambient conditions. CDs are regarded as a new class of pharmaceutical excipients, and have been proven efficient in the formulation of inclusion complexes of drug compounds. ${ }^{26}$ Ionic liquids are typically regarded as green solvents, by virtue of their negligible vapour pressure, and are slowly gaining importance in the pharmaceutical field as solvents. ${ }^{27,28}$ This thesis covers the use of non-ambient techniques, especially high pressure, to investigate 1) CD inclusion complex formation with drug molecules, 
and 2) the solid-state behaviour of commonly used ILs using non-ambient crystallisation conditions, and subsequently using them as potential crystallisation solvents for drug molecules.

\subsection{Materials and Methods}

\subsubsection{Materials}

Each material used in this work is described in detail in the appropriate section of each chapter.

\subsubsection{Methods}

Different analytical methods are used in pharmaceutical science to explore the polymorphic behaviour of drug molecules and the formation of multicomponent crystals. $5,10,12,29$ Methods can be broadly classified as follows: a) thermal methods such as thermogravimetry, differential scanning calorimetry or differential thermal analysis; b) vibrational spectroscopic methods such as Raman, infrared and UV-Vis spectroscopy; c) nuclear magnetic resonance spectroscopy in both solution and solid states; d) solubility or dissolution studies; e) microscopy methods such as polarised optical microscopy, often coupled with a hot/cooling stage, scanning electron microscopy; f) X-ray diffraction methods for the analysis of powder and single-crystal samples.

Single-crystal X-ray diffraction is the method of choice when it comes to unveiling the structure at the atomic level of novel physical forms. Accurate and precise knowledge of atomic coordinates enables in depth studies of a structure beyond atomic connectivity and the derivation of structure-property relationships. For instance, it enables detailed studies of hydrogen-bonding patterns and other intermolecular interactions; it enables the calculation of lattice energies and intermolecular interaction energies by means of more sophisticated computational studies, e.g. by DFT of semi-empirical methods. ${ }^{30-32}$

In this work, experimental investigations of novel physical forms of drug molecules has been carried out primarily by means of X-ray diffraction on single crystals at high pressure in a DAC, or at low temperature at ambient pressure. The studies made extensive use of polarised optical microscopy (POM) at variable temperature conditions and in some cases of Raman spectroscopy as a fingerprinting technique. Single-crystal X-ray diffraction is a very wellestablished technique and numerous books are dedicated to this subject. Each book provides ample details of the theory of diffraction as well as principles and practice of data collection data reduction, structure solution and structure refinement. References 33-38 provide excellent starting 
points for further reading. In this thesis, details of data collection strategies, structure solution and refinement are given in full details in each relevant chapter.

\subsubsection{High-pressure crystallography}

Since the pioneering work of Bridgman, ${ }^{39,40}$ a series of multiple innovations have had a tremendous impact on the scope of high-pressure research and the feasibility and ease of performing high-pressure experiments. As described by several authors, ${ }^{41-44}$ some of the principal milestones include a) the design and production of DACs in the 1950s, 45,46 which rapidly became an easy-to-use multidisciplinary tool for discovering new states of matter; b) the introduction of the pressure calibration by the ruby fluorescence method and the advent of Merrill-Bassett DACs in the 1970s; ${ }^{47}$ c) advances in instrumentation (e.g. the Paris-Edinburgh cell for neutron diffraction) and data collection strategies at large scale facilities and in the laboratory starting from the 1990s. High-pressure crystallography on organic materials became facile and spread rapidly after the introduction of area detectors in the late 1990s..$^{48}$ Applying pressure on organic liquids or materials in solutions was quickly revealed to be a very effective approach for studying crystal polymorphism in a wide range of molecular compounds, including drugs and biomolecules. ${ }^{15-17}$
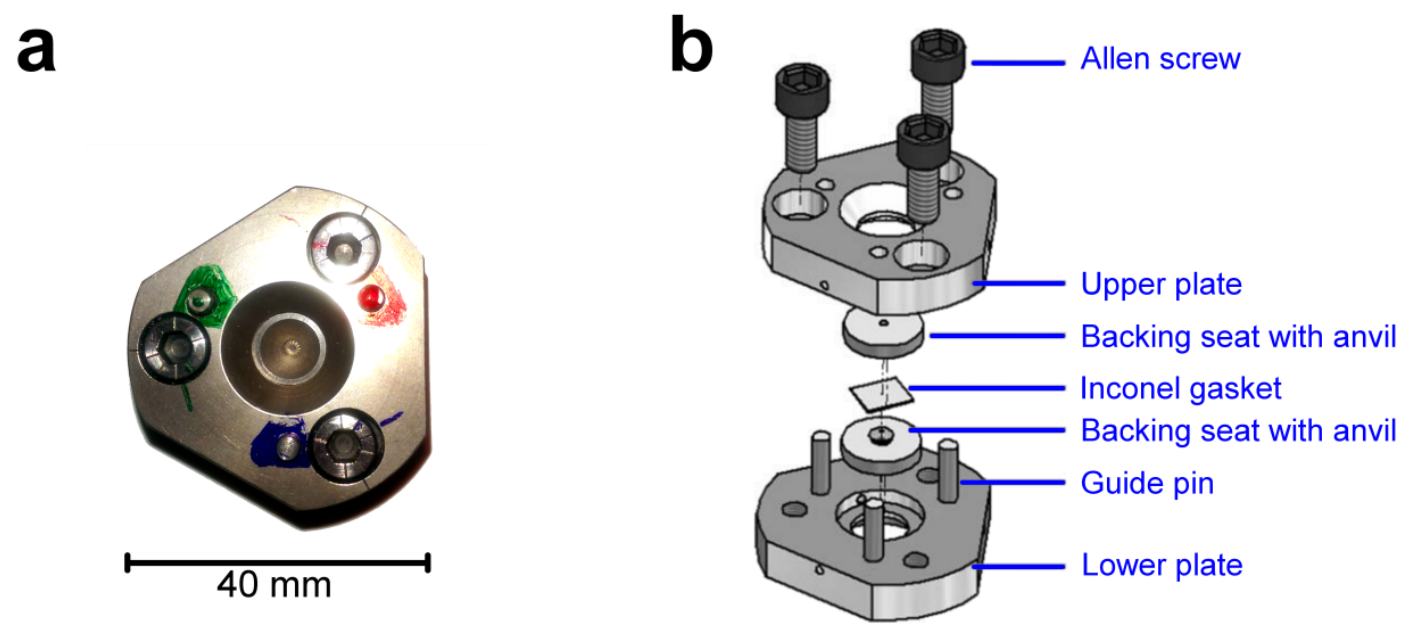

Figure 1.4. A Merrill-Bassett DAC. a) photographic image with colour coded guide pins and Allen screws; b) Exploded view of (a) modified from reference ${ }^{22}$.

In a typical DAC two diamonds, acting as anvils, press on a metallic gasket; the gasket contains a cylindrical hole, which is the sample chamber. The diamonds are supported by two metallic plates, called backing seats usually made of steel or tungsten carbide in modern cell designs. Each seat is supported by a plate made of steel or another strong alloy. The plates are designed so that one can be secured to the other one through the use of screws. Tightening the screws has the effect of bringing the two diamonds close together, thereby generating high pressure inside the sample chamber. Figure 1.4 represents one of most common DACs, namely a 
Merrill-Bassett DAC. ${ }^{19,22}$ For more details about DACs the reader should consult Miletich et al. ${ }^{49}$ and Katrusiak ${ }^{16}$, which offer comprehensive reviews of the different types of DACs available, their uses and physics of X-ray diffraction experiments using DACs. ${ }^{49}$ The different types of DACs that have been used throughout this work and the related technical details, as well as those of the X-ray diffraction studies, are described as appropriate in each chapter.

\subsubsection{Raman spectroscopy}

It is beyond the scope of this introduction to give a detailed account of Raman spectroscopy; references 50-52 provide a good starting point for further reading. Raman spectroscopy enables a rapid qualitative appreciation of the composition and purity of matter. The technique is based on exciting matter with monochromatic radiation, usually a laser in the visible region, and collecting the scattered light using a CCD detector. When interacting with the sample, most of the light is scattered without modification of the wavelength; this radiation is called Rayleigh scattering and is filtered out using a notch filter. A small portion of the light is scattered inelastically, giving rise to the Raman effect, and collected on the CCD detector after being split by a diffraction grating (Figure 1.5).

In the Raman effect, molecules respond to the collision with the radiation by vibrating. The vibration or stretching is exclusive to the chemical nature of the atoms and bonds within the matter. ${ }^{51}$ Hence, Raman spectroscopy is a very sensitive technique; the complexity of a Raman spectrum is proportional to the complexity of the chemical species within the sample. In contrast to IR spectroscopy, collection of a Raman spectrum requires no sample preparation; modern Raman spectrometers are usually coupled with high-resolution microscopes and can easily accommodate a large variety of sample environments, such as DACs and heating/cooling stages.

In this thesis, Raman spectroscopy has been used as a fingerprinting method in the investigation of the polymorphic behaviour of ionic liquids, and contributed to a qualitative interpretation of the stoichiometry of inclusion complexes at high pressure. Experimental details are described in more details in the relevant chapters (Chapter 4 and Chapter 7). 


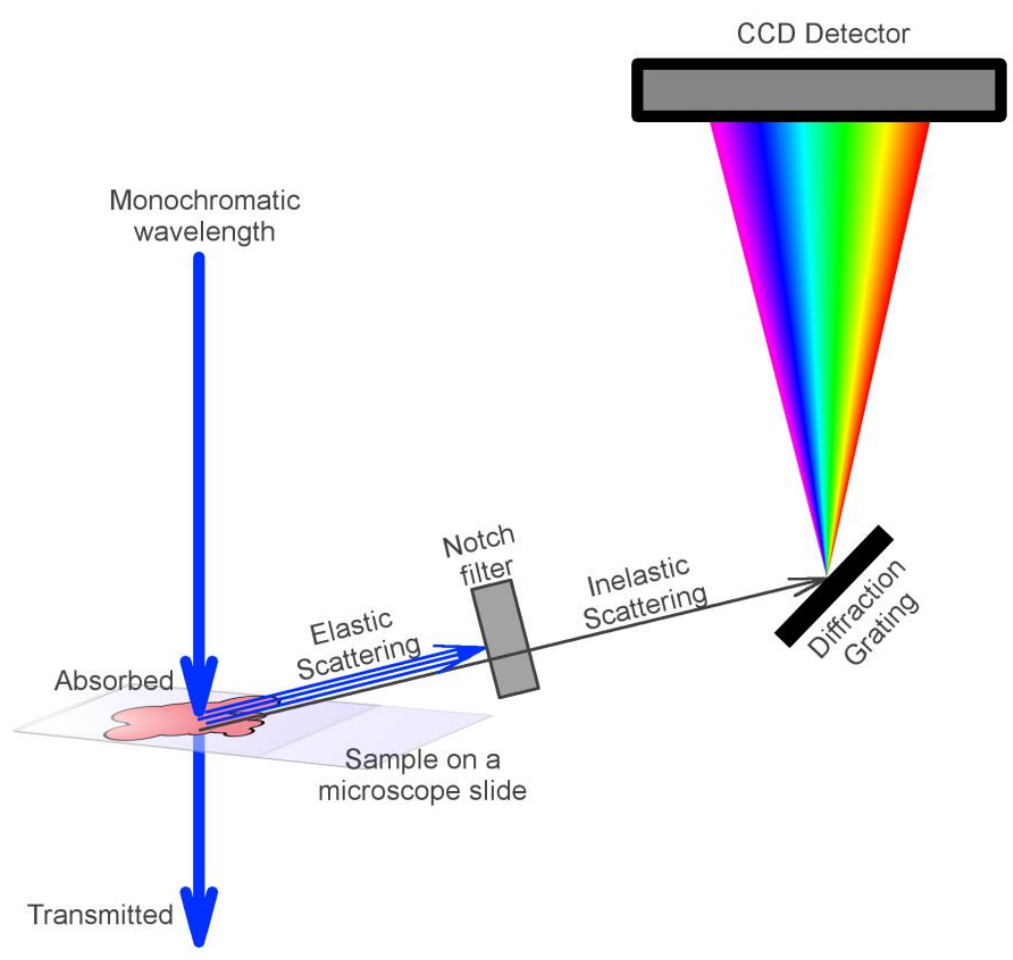

Figure 1.5. Cartoon summarising the Raman spectroscopy technique.

\subsubsection{Heating and cooling stage microscopy}

Heating and cooling stage microscopy is a microanalytical method combining optical microscopy with temperature control. ${ }^{53}$ Hot stage microscopy can be qualitatively compared to a micrographic DSC device. ${ }^{54,55}$ The sample is introduced into the stage and placed on a small heating block equipped with a glass window. Because of the electrical resistance of the block, the latter can be easily heated by applying electrical current. The temperature of the block is measured via a thermocouple. Cooling can be achieved by applying a stream of cold nitrogen going through a tubular gallery within the block; an alternative gas port uses the same nitrogen stream to replace the atmospheric air within the stage. An automatic and quick alternation between heating and cooling enables accurate temperature control.

The history of heating and cooling stages as well as the advances of the techniques have been well documented in the literature. ${ }^{53,54,56}$ Details on the experimental setup are described in the relevant chapters (Chapters 7 and Chapter 8).

\subsubsection{Other experimental methods}

Sample handling differs from one compound to another; for each of the compounds studied detailed and tailor-made crystallisation protocols have been established. These are described in the experimental section of each chapter. 


\subsection{References}

(1) Burchell, T. D. Carbon Materials for Advanced Technologies, 1st ed.; Elsevier Science, 1999.

(2) Ströck, M. Eight Allotropes of Carbon (from the Wikipedia Commons) https://en.wikipedia.org/wiki/File:Eight_Allotropes_of_Carbon.png (accessed Aug 16, 2015).

(3) Haleblian, J.; McCrone, W. Pharmaceutical Applications of Polymorphism. J. Pharm. Sci. 1969, $58(8), 911-929$.

(4) IUCr. Online Dictionary of Crystallography - IUCr (accessed 13/08/2015) http://reference.iucr.org/dictionary/Polymorphism.

(5) Bernstein, J. Polymorphism in Molecular Crystals, 1st ed.; Oxford University Press: Oxford, United Kingdom, 2002.

(6) Bauer, J.; Spanton, S.; Henry, R.; Quick, J.; Dziki, W.; Porter, W.; Morris, J. Ritonavir: An Extraordinary Example of Conformational Polymorphism. Pharm. Res. 2001, 18 (6), 859-866.

(7) Morissette, S. L.; Soukasene, S.; Levinson, D.; Cima, M. J.; Almarsson, O. Elucidation of Crystal Form Diversity of the HIV Protease Inhibitor Ritonavir by High-Throughput Crystallization. Proc. Natl. Acad. Sci. U. S. A. 2003, 100 (5), 2180-2184.

(8) Bučar, D.-K.; Lancaster, R. W.; Bernstein, J. Disappearing Polymorphs Revisited. Angew. Chemie Int. Ed. 2015, 54 (24), 6972-6993.

(9) Hilfiker, R. Polymorphism in the Pharmaceutical Industry, 1st ed.; WILEY-VCH Verlag GmbH \& Co.: Weinheim, 2006.

(10) Hilfiker, R.; Blatter, F.; von Raumer, M. Relevance of Solid-Stete Properties for Pharmaceutical Products. In Polymorphism: in the Pharmaceutical Industry; Hilfiker, R., Ed.; WILEY-VCH Verlag GmbH \& Co. KGaA: Weinheim - Germany, 2006; pp 1-17.

(11) Karpinski, P. H. Polymorphism of Active Pharmaceutical Ingredients. Chem. Eng. Technol. 2006, 29 (2), 233-237.

(12) Lee, E. H. A Practical Guide to Pharmaceutical Polymorph Screening \& Selection. Asian J. Pharm. Sci. 2014, 9 (4), 1-13.

(13) Lin, S. Y. An Overview of Famotidine Polymorphs: Solid-State Characteristics, Thermodynamics, Polymorphic Transformation and Quality Control. Pharm. Res. 2014, 31 (7), 1619-1631.

(14) Braga, D.; Grepioni, F.; Maini, L. The Growing World of Crystal Forms. Chem. Commun. (Camb). 2010, 46 (34), 6232-6242.

(15) Fabbiani, F. P. A.; Pulham, C. R. High-Pressure Studies of Pharmaceutical Compounds and Energetic Materials. Chem. Soc. Rev. 2006, 35 (10), 932-942.

(16) Katrusiak, A. High-Pressure Crystallography. Acta Crystallogr. A. 2008, 64, 135-148.

(17) Boldyreva, E. V. Multicomponent Organic Crystals at High Pressure. Zeitschrift für Krist. - 
Cryst. Mater. 2014, 229, 236-245.

(18) Boldyreva, E. High-Pressure Polymorphs of Molecular Solids: When Are They Formed, and When Are They Not? Some Examples of the Role of Kinetic Control. Cryst. Growth Des. 2007, 7 (9), 1662-1668.

(19) Merrill, L.; Bassett, W. A. Miniature Diamond Anvil Pressure Cell for Single Crystal X-Ray Diffraction Studies. Rev. Sci. Instrum. 1974, 45, 290-294.

(20) Allan, D. R.; Miletich, R.; Angel, R. J. A Diamond-Anvil Cell for Single-Crystal X-Ray Diffraction Studies to Pressures in Excess of 10 GPa. Rev. Sci. Instrum. 1996, 67 (3), 840-842.

(21) Ahsbahs, H. New Pressure Cell for Single-Crystal X-Ray Investigations on Diffractometers with Area Detectors. Zeitschrift für Krist. 2004, 219 (6-2004), 305-308.

(22) Moggach, S. A.; Allan, D. R.; Parsons, S.; Warren, J. E. Incorporation of a New Design of Backing Seat and Anvil in a Merrill-Bassett Diamond Anvil Cell. J. Appl. Crystallogr. 2008, 41 (2), 249-251.

(23) Billing, D. G.; Katrusiak, A. Non-Ambient Crystallography - Is Extreme Becoming Common? Acta Crystallogr. Sect. B Struct. Sci. Cryst. Eng. Mater. 2014, 70 (3), 399-400.

(24) Angel, R.; Bouvier, P.; Fabbiani, F. P. A. Preface - Special Issue on High Pressure. Zeitschrift für Krist. - Cryst. Mater. 2014, 229 (2).

(25) Hejny, C.; Minkov, V. S. High-Pressure Crystallography of Periodic and Aperiodic Crystals. IUCrJ 2015, 2 (2), 218-229.

(26) Loftsson, T.; Brewster, M. E.; Rewster, M. A. E. B. Pharmaceutical Applications of Cyclodextrins. 1. Drug Solubilization and Stabilization. J. Pharm. Sci. 1996, 85 (10), 10171025.

(27) Mizuuchi, H.; Jaitely, V.; Murdan, S.; Florence, a. T. Room Temperature Ionic Liquids and Their Mixtures: Potential Pharmaceutical Solvents. Eur. J. Pharm. Sci. 2008, 33 (4-5), 326-331.

(28) Smith, K. B.; Bridson, R. H.; Leeke, G. A. Solubilities of Pharmaceutical Compounds in Ionic Liquids. J. Chem. Eng. Data 2011, 56, 2039-2043.

(29) Giron, D. Investigations of Polymorphism and Pseudo-Polymorphism in Pharmaceuticals by Combined Thermoanalytical Techniques. J. Therm. Anal. Calorim. 2001, 64, 37-60.

(30) Arunan, E.; Desiraju, G. R.; Klein, R. a.; Sadlej, J.; Scheiner, S.; Alkorta, I.; Clary, D. C.; Crabtree, R. H.; Dannenberg, J. J.; Hobza, P.; et al. Defining the Hydrogen Bond: An Account (IUPAC Technical Report). Pure Appl. Chem. 2011, 83 (8), 1619-1636.

(31) Stone, A. The Theory of Intermolecular Forces, 2nd ed.; Oxford University Press: Oxford, United Kingdom, 2013.

(32) Rissanen, K. Advanced X-Ray Crystallography; Springer-Verlag Berlin Heidelberg: Berlin Heidelberg, Germany, 2012.

(33) Giacovazzo, C.; Monaco, H. L.; Viterbo, D.; Scordari, F.; Gilli, G.; Zanotti, G.; Catti, M. Fundamentals of Crystallography; Giacovazzo, C., Ed.; Oxford University Press: New York, 1997; Vol. 198. 
(34) Massa, W. Crystal Structure Determination, 2nd ed.; Springer-Verlag Berlin Heidelberg: Berlin - Heidelberg, Germany, 2004.

(35) Müller, P.; Herbst-Irmer, R.; Spek, A.; Schneider, T.; Sawaya, M. Crystal Structure Refinement: A Crystallographer's Guide to SHELXL; Oxford University Press: Oxford, United Kingdom, 2006.

(36) Clegg, B.; Cooper, R.; Copley, R. C. B.; Howard, J. A. K.; Palatinus, L.; Parsons, S.; Sivia, D. X-Ray Structure Analysis - 14th BCA/CCG Intensive Teaching School on X-Ray Structure Analysis; Trevelyan College, Durham, UK: Durham, IK, 2012.

(37) Glusker, J. P.; Trueblood, K. N. Crystal Structure Analysis: A Primer, 3rd ed.; Oxford University Press: Oxford, United Kingdom, 2010.

(38) Borchard-Ott, W. Crystallography: An Introduction, 3rd ed.; Springer-Verlag Berlin Heidelberg: Berlin - Heidelberg, Germany, 2012.

(39) Bridgman, P. W. The Physics of High Pressure; Dover Publications: London, United Kingdom, 1931.

(40) Bridgman, P. W. Recent Work in the Field of High Pressures. Rev. Mod. Phys. 1946, 18 (1), 193.

(41) Jayaraman, A. Diamond Anvil Cell and High-Pressure Physical Investigations. Rev. Mod. Phys. 1983, 55 (1), 65-108.

(42) Hazen, R. M.; Downs, R. T. High-Temperature \& High-Pressure Crystal Chemistry (Reviews in Mineralogy and Geochemistry Volume 41); Mineralogical Society of America: Chantilly, United States, 2001.

(43) Hazen, R. M.; Finger, L. W. Comparative Crystal Chemistry: Temperature, Pressure, Composition, and the Variation of Crystal Structure, 1st ed.; Finger, L. W., Ed.; John Wiley \& Sons Ltd: New Jersey, 1982.

(44) McMahon, M. I. High Pressure Diffraction from Good Powders, Poor Powders and Poor Single Crystals. In High-pressure Crystallography; Katrusiak, A., McMillan, P., Eds.; Springer Netherlands: Dordrecht, Netherlands, 2004; pp 1-20.

(45) Jamieson, J. C.; Lawson, A. W.; Nachtrieb, N. D. New Device for Obtaining X-Ray Diffraction Patterns from Substances Exposed to High Pressure. Rev. Sci. Instrum. 1959, 30 (11), 1016-1019.

(46) Weir, C. E.; E. R. Lippincott; Valkenburg, A. Van; Bunting, E. N. Infrared Studies in the 1- to 15-Micron Region to 30,000 Atmospheres. J. Reasearch Natl. Bur. Stand. - A. Phys. Chem. 1959, $63 A(1), 55-62$.

(47) Piermarini, G. J.; Block, S.; Barnett, J. D.; Forman, R. A. Calibration of the Pressure Dependence of the R1 Ruby Fluorescence Line to 195 Kbar. J. Appl. Phys. 1975, 46 (6), 27742780 .

(48) Boldyreva, E. V.; Dera, P. High-Pressure Crystallography: From Fundamental Phenomena to Technological Applications, 1st ed.; Springer Netherlands: Dordrecht, Netherlands, 2010.

(49) Miletich, R.; Allan, D. R.; Kuhs, W. F. High-Pressure Single-Crystal Techniques. In High- 
Temperature \& High-Pressure Crystal Chemistry (Reviews in Mineralogy and Geochemistry Volume 41); Hazen, R. M., Downs, R. T., Eds.; Mineralogical Society of America: Chantilly, United States, 2001; pp 445-519.

(50) Schrader, B. Infrared and Raman Spectroscopy: Methods and Applications, 1st ed.; VCH: Weinheim, Germany, 1995.

(51) Colthup, N. B.; Daly, L. H.; Wiberley, S. E. Introduction to Infrared and Raman Spectroscopy, 3rd ed.; Academic Press, Inc.: San Diego, 1990.

(52) Ferraro, J. R.; Nakamoto, K.; Brown, C. W. Introductory Raman Spectroscopy, 2nd ed.; Academic Press, Inc.: Waltham, 2003.

(53) McCrone, W. C. Fusion Methods in Chemical Microscopy; Interscience Publishers Inc.: New York, United States of America, 1957.

(54) Vitez, I. M.; Newman, A. W.; Davidovich, M.; Kiesnowski, C. The Evolution of Hot-Stage Microscopy to Aid Solid-State Characterizations of Pharmaceutical Solids. Thermochim. Acta 1998, 324 (1-2), 187-196.

(55) Bakar, M. R. A.; Nagy, Z. K.; Rielly, C. D. A Combined Approach of Differential Scanning Calorimetry and Hot-Stage Microscopy with Image Analysis in the Investigation of Sulfathiazole Polymorphism. J. Therm. Anal. Calorim. 2010, 99 (2), 609-619.

(56) McCall, J. L.; French, P. M. Interpretive Techniques for Microstructural Analysis, 1st Ed.; Springer US: New York, United States of America, 1977. 


\section{PART I.}

\section{Exploring Inclusion Complex Formation of Cyclodextrins with Drug Molecules}




\section{Chapter 2. Introduction to Part I}

\subsection{Introduction}

Native cyclodextrins (CDs) are cyclic oligosaccharides composed of six or more units of $\alpha-$ D-glucopyranose linked by $\alpha-(1 \rightarrow 4)$ glycosidic bonds (Figure 2.1). Depending on the number of glucopyranose units, native CDs have been classified as $\alpha-, \beta$ - and $\gamma$-CD having 6, 7 and 8 pyranose units, respectively. A CD molecule has a hollow truncated cone-like shape where the primary hydroxy groups sit on the narrow side of the truncated cone and the secondary ones on the wide side (Figure 2.1). The architecture of CDs molecules favours the partition of the structure into an outer surface and an inner cavity, which are hydrophilic and hydrophobic in character, respectively (Figure 2.2). ${ }^{1} \alpha$ - and $\gamma$-CDs have considerably higher water solubility compared to $\beta$ $\mathrm{CD}$, with values of 145,232 , and $18.5 \mathrm{~g} / \mathrm{L}$, respectively. ${ }^{2}$ The very low water solubility of $\beta$-CD has been ascribed to the formation of intramolecular hydrogen bonds between glucose molecules in solution. ${ }^{3}$ Chemical modifications, mainly by methylation or hydroxypropylation, have been applied on CDs to improve their water solubility creating so-called CDs derivatives.
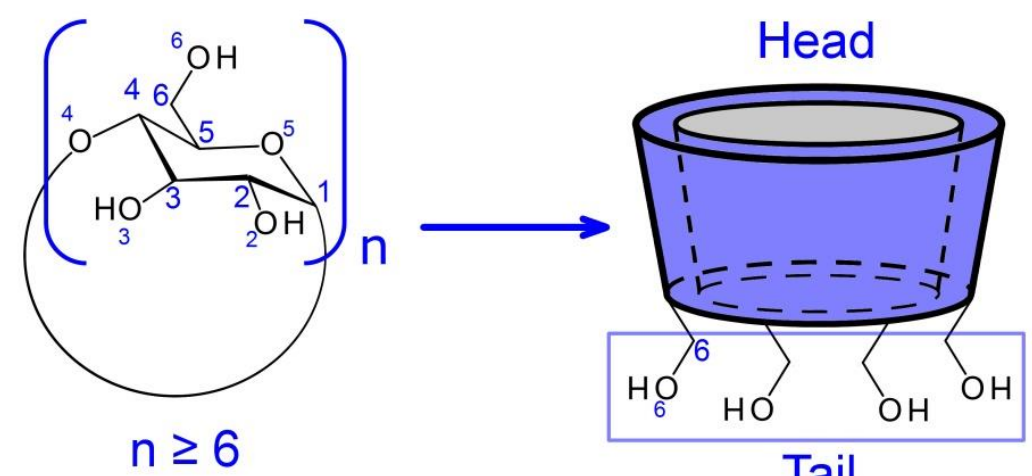

Tail

Figure 2.1. Chemical diagram of $\alpha$-D-glucopyranose unit (left) and the general shape of a CD molecule (right)

CDs were extracted for the first time in 1891 by Villiers while studying the bacterial transformation of starch into dextrin by Bacillus amylobacter: ${ }^{\mathrm{C}} \mathrm{CD}$ s were thought to be the product of the fermentation of starch, and were named "dextrin precipitate" by Villiers. Twelve years later, Schardinger, while studying thermophilic bacteria producing the same "cellulosine" as a product of degradation of starch, reported that Villiers' bacterial cultures were not pure, and "cellulosine" or "crystalline dextrin" was produced by Bacillus macerans. ${ }^{5}$ This marked the beginning of a pioneering work on "Schardinger sugars" initiated by Schardinger and undertaken later on by several research groups. The first report on inclusion complex formation was a suggestion made by Cramer in the 1940s, which was equally accepted and disapproved by the chemical community. ${ }^{6}$ Cramer followed his idea and proved by experimental means not only that "Schardinger sugars" 
form inclusion complexes, but also that they are enantiomer selective and have catalytic properties. These findings started a worldwide interest in "cyclodextrins" and especially in inclusion complex formation. Cramer's work has been well summarised and referenced by Stoddart in 1989.7

In 1957, French reported that a Schardinger-sugar-based diet on rats caused their death within a week. ${ }^{8}$ This led to a temporary distrust of the scientific community towards cyclodextrins for using them as prominent drug carriers. Fortunately, Anderson et al. counter-published a thorough toxicological study on rats, based on a marked ${ }^{14} \mathrm{C}$-cyclodextrins diet, showing that native cyclodextrins are safer than initially thought, and that the initial report about lethality was due to insufficient food intake. ${ }^{9}$ This triggered a particular interest in CD application as drug carrier and pioneering work in this area was undertaken by different groups, notably by Saenger's in Germany. ${ }^{10}$

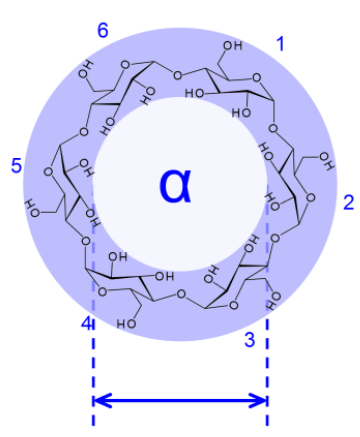

$4.7-5.3 \AA$

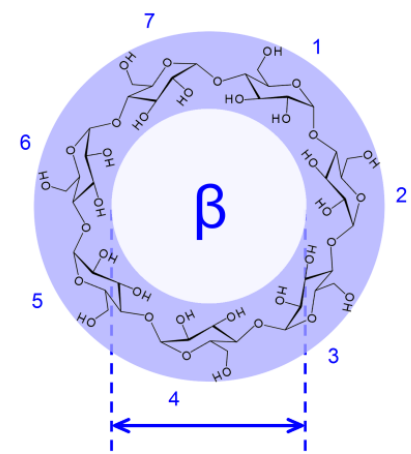

$6.0-6.5 \AA$

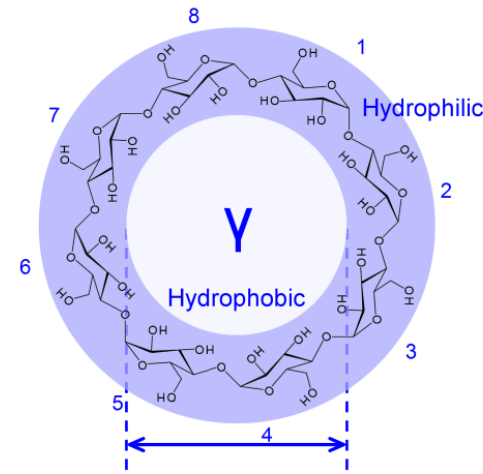

$7.5-8.3 \AA$

Figure 2.2. Chemical diagram of $\alpha-, \beta-$ and $\gamma-C D$

The advances in biotechnology and genetic engineering of the 1970s facilitated and boosted CD production. Specifically, CD were found to form due to an enzymatic degradation of starch by the family of cyclodextrin glycosyltransferase found in several bacterial species. ${ }^{11}$ The type of CD formation can be oriented depending on the origin of the enzyme, ${ }^{11}$ but more commonly through the purification process by either 1) precipitation, 2) chromatography/adsorption techniques, or 3) filtration. ${ }^{11}$ Purification by precipitation is the easiest process and particularly elective for the production of $\beta$-CD taking advantage of its low solubility in water. ${ }^{2}$ These advances caused a drop in CDs' prices; as the compounds became more accessible, their properties were explored and exploited by an increasing number of research groups. This translated into a tremendous inflation in the number of CD-based-literature since the 1980s. For further reading, Szejtli summarised nicely the history of cyclodextrins in two of his reviews.,12

Nowadays, CDs find a broad application and are mostly attractive thanks to their inclusion complex formation property. They are for instance used in a) the pharmaceutical industry to enclose drug molecules and indirectly improve their solubility and bioavailability; b) the food 
industry as masks of taste and smell, and as flavour protectors; c) the chemical industry as catalysts and retention agents in chromatography; d) cosmetics as stabiliser. Martin del Valle reported an extensive list of CDs properties and applications..$^{13}$

In 1976, $\alpha$ - and $\beta$-CD were approved as food additives in Japan and within the same year the first CD-based pharmaceutical products, encapsulated prostaglandins ${ }^{14}$, were commercially available. ${ }^{15}$ Nowadays, CDs are used worldwide and a non-exhaustive list of CD-based products can be found in the references by Szejtli16 as well as Loftsson and Duchêne. ${ }^{15}$

The literature reveals, to the best of our knowledge, that structural studies of CDs and CD inclusion complexes have always been carried out using ambient-pressure techniques. Highpressure techniques have been shown to be a suitable method for obtaining novel crystal forms of pharmaceutical compounds, in particular for exploring the phenomenon of polymorphism and solvate formation more thoroughly. ${ }^{17,18}$ Investigating inclusion complex formation of cyclodextrins would be an interesting extension of the technique: can cyclodextrin complex formation be achieved at high pressure or would individual crystal components crystallise separately, and if so, which ones? In this section, examples of successful in-situ complex formation as well as unexpected results are presented for $\alpha$ - and $\beta$-cyclodextrin with active pharmaceutical ingredients using water as crystallisation medium. 


\subsection{References}

(1) Loftsson, T.; Brewster, M. E.; Rewster, M. A. E. B. Pharmaceutical Applications of Cyclodextrins. 1. Drug Solubilization and Stabilization. J. Pharm. Sci. 1996, 85 (10), 10171025.

(2) French, D.; Levine, M. L.; Pazur, J. H.; Norberg, E. Studies on the Schardinger Dextrins; the Preparation and Solubility Characteristics of Alpha, Beta and Gamma Dextrins. J. Am. Chem. Soc. 1949, 71 (1), 353-356.

(3) Szejtli, J. Introduction and General Overview of Cyclodextrin Chemistry. Chem. Rev. 1998, 98, 1743-1753.

(4) Villiers, M. A. Sur La Transformation de La Fécule En Dextrin Par Le Ferment Butyrique. Comptes rendus des séances l'académie des Sci. 1891, 435-437.

(5) Schardinger, F. Über Thermophile Bakterien Aus Verschiedenen Speisen Und Milch, Sowie Über Einige Umsetzungsprodukte Derselben in Kohlenhydrathaltigen Nährlösungen, Darunter Krystallisierte Polysaccharide (Dextrine) Aus Stärke. Zeitschrift für Untersuchung der Nahrungs- und Genußm. 1903, 19, 865-880.

(6) Cramer, F. Cyclodextrin - A Paradigmatic Model. In Proceedings of the First International Symposium on Cyclodextrins; Szejtli, J., Ed.; Springer Science+Business Media: Budapest, Hungary, 1981; pp 3-14.

(7) Stoddart, J. F. A Century of Cyclodextrins. Carbohydr. Res. 1989, 192, xii - xv.

(8) French, D. The Schardinger Dextrins. Adv. Carbohydr. Chem. 1957, 12, 189-260.

(9) Andersen, G. H.; Robbins, F. M.; Domingues, F. J.; Moores, R. G.; Long, C. L. The Utilization of Schardinger Dextrins by the Rat. Toxicol. Appl. Pharmacol. 1963, 5, 257-266.

(10) Saenger, W. Cyclodextrin Inclusion Compounds in Research and Industry. Angew. Chemie Int. Ed. English 1980, 19 (5), 344-362.

(11) Biwer, A.; Antranikian, G.; Heinzle, E. Enzymatic Production of Cyclodextrins. Appl. Microbiol. Biotechnol. 2002, 59, 609-617.

(12) Szejtli, J. Past, Present and Futute of Cyclodextrin Research. Pure Appl. Chem. 2004, 76 (10), 1825-1845.

(13) Martin Del Valle, E. M. Cyclodextrins and Their Uses: A Review. Process Biochem. 2003, 39 (9), 1033-1046.

(14) Inaba, K.; Wakuda, T.; Uekama, K. Prostaglandins and Their Cyclodextrin Complexes. J. Incl. Phenom. 1984, 2 (3-4), 467-474.

(15) Loftsson, T.; Duchêne, D. Cyclodextrins and Their Pharmaceutical Applications. Int. J. Pharm. 2007, 329 (1-2), 1-11.

(16) Szejtli, J. Cyclodextrins: Applications. In Encyclopedia of Supramolecular Chemistry, volume 1; Atwood, J. L., Steed, J. W., Eds.; CRC Press: New York, 2004; pp 405-413.

(17) Fabbiani, F. P. A.; Pulham, C. R. High-Pressure Studies of Pharmaceutical Compounds and Energetic Materials. Chem. Soc. Rev. 2006, 35 (10), 932-942.

(18) Boldyreva, E. V. High-Pressure Diffraction Studies of Molecular Organic Solids. A Personal View. Acta Crystallogr. A. 2008, 64 (Pt 1), 218-231. 


\section{Chapter 3. $\alpha$-Cyclodextrin -succinic acid inclusion complex: a novel packing type of $\alpha$-cyclodextrin}

\subsection{Synopsis}

This chapter describes a new packing type of $\alpha$-cyclodextrin inclusion complexes, obtained here with succinic acid at low-temperature crystallisation conditions. The new structure is characterised by heavy disorder of the guest, the solvent, and part of the host molecules. The complex could not be obtained by high-pressure crystallisation, an observation which may be closely related to the disordered nature of the structure.

\subsection{Introduction}

The work presented here is part of an ongoing investigation of cyclodextrin inclusion complex and hydrate formation at both ambient- and high-pressure crystallisation conditions using water as crystallisation medium. ${ }^{1,2} \alpha-\mathrm{CD}$, the smallest natural cyclic oligosaccharide with six sugar units, was adopted for studying and comparing inclusion complex formation with small molecules (molecular weight $<500$ Daltons), ${ }^{3,4}$ regardless of their water solubility, in order to explore the effect of pressure on the complexation and decomplexation processes.

Succinic acid (SA), an aliphatic dicarboxylic acid, is essential in aerobic cellular metabolism by intervening in the citric acid cycle, a metabolic pathway for the regeneration of adenosine triphosphate (ATP), which is the main energy source of most cellular functions. ${ }^{5}$

The structure of a $\beta$-CD.SA inclusion complex (CSD refcode KUJSEC) has been previously obtained while investigating the enhancement of succinic anhydride's reactivity using $\beta-C D$ as molecular cages in aqueous solutions. ${ }^{6}$ We found that this complex can easily be obtained with SA instead of succinic anhydride (See Chapter 5); the large cavity size of $\beta-C D$, which is $6.0-6.5 \AA$ in diameter, ${ }^{7}$ can easily accommodate SA and the crystal structure of the complex shows several intermolecular interactions between host, guest and solvent molecules. ${ }^{6}$ We hypothesised that with a width [5.265(13) $\AA$, see fig. 1] commensurable with the cavity diameter of $\alpha$-CD (4.7-5.3 $\AA$ ), 7 SA could in principle form a crystalline complex with $\alpha$-CD. The literature shows that similar linear compounds form inclusion complexes with $\alpha$-CD (see for example CSD refcodes BUPDEV, 8 $\mathrm{CDKABA}^{9}$ and $\mathrm{XIGBOE}^{10}$ ). Exploration of the $\mathrm{SA} / \alpha-\mathrm{CD} /$ water system is interesting for investigating complex formation at both ambient- and high-pressure conditions and, if successful, for comparing the structures of $\alpha$-CD· and $\beta$-CD·SA inclusion complexes.

Three packing types have been described for both CD hydrates and inclusion complexes: two of these belong to the cage type and are known as herringbone- and brick-type cages, and the 
third one is the channel type. ${ }^{11}$ The packing preference of CD molecules for one of these types is closely related to the size and shape of the guest molecule.11 A Cambridge Structural Database ${ }^{12}$ (CSD) search (the CSD, V. 5.36 including updates to Nov. 2014 was searched for structures with 3D coordinates) shows that $\alpha$-CD crystallises in the channel packing type in $52 \%$ of the total 96 hits, followed by the brick-type in $26 \%$ and the herringbone-type in $22 \%$ of the structures. The channelpacking type of the structures is favoured by $\alpha-\mathrm{CD}$ small and narrow size of the cavity: most guest molecules are inserted lengthwise into CDs cavities and form infinite columns. ${ }^{13}$

(a)

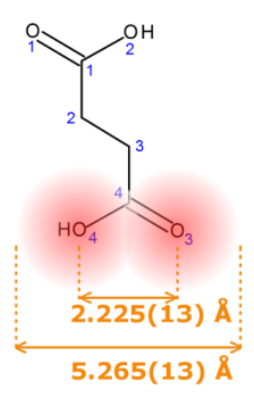

(b)

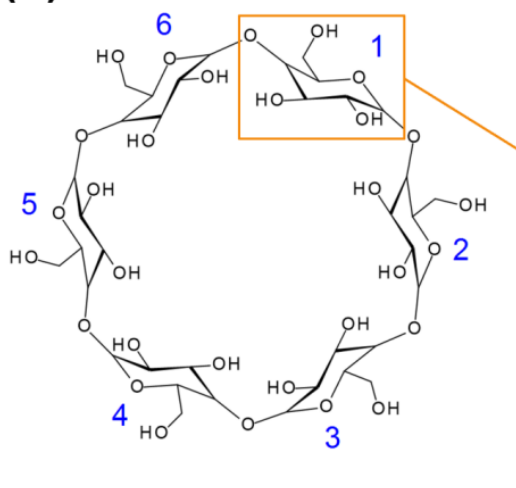

(c)

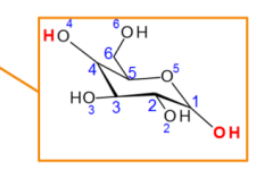

Figure 3.1. Diagrams and numbering schemes of: (a) succinic acid: the meanO3-04 distance was computed from 72 structures of SA in the CSD, the width of SA is the [meano3-04 $\left.+2 \times\left(r_{\mathrm{vdw}}\right) \mathrm{o}\right]^{14} ;$ (b) $\alpha$-CD and (c) $\alpha$-Dglucopyranose

\subsection{Experimental}

\subsubsection{Ambient-pressure crystallisation}

Both $\alpha$-CD and SA were bought from Fluka and SIGMA, respectively, and used without further treatment. An undersaturated solution with a 1:1 molar ratio mixture of $\alpha$-CD (194.57 mg) and succinic acid $(23.6 \mathrm{mg})$ was prepared in approximately $2 \mathrm{ml}$ of demineralised water. Fast evaporation of the solution yielded a glass at ambient condition. A combination of cold temperatures ( $c a .277 \mathrm{~K}$ ) and very slow evaporation over the course of six months led to the crystallisation of hexagonal prism-shaped crystals (Figure 3.2.a). When the experiment was repeated using a more concentrated solution, crystallisation occurred within a week at the same low-temperature conditions. X-ray data were collected on a single crystal specimen at $90 \mathrm{~K}$.

\subsubsection{Multi-temperature experiment}

A subsequent experiment was performed at low-temperature conditions using the same procedure described above on a second single-crystal specimen. Data sets were collected on the same single crystal using the same data collection strategy at 100, 120, 150, 180, 210, 240 and 270. 
The purpose of this experiment was to monitor structural changes, i.e. phase transitions, and possible crystal decay as function of temperature.

\subsubsection{High-pressure crystallisation}

The high-pressure experiment was carried out using a Merrill-Bassett diamond anvil cell (DAC), ${ }^{15}$ with a half opening angle of $45^{\circ}$. The DAC was equipped with $800 \mu \mathrm{m}$ culet diamonds of low fluorescence grade and Inconel gaskets with a starting diameter hole of $350 \mu \mathrm{m}$. Pressure was monitored using the ruby fluorescence method described by Piermarini et al. ${ }^{16}$ Several experiments were performed as detailed below. In all experiments it was observed that the initial crystal undergoes dissolution as function of increasing pressure, temperature or both. Hence, all experiments aimed at compressing crystals grown at ambient pressure past their dissolution pressure in the hope of triggering a crystallisation event. The first experiment consisted of loading a crystal of the complex grown at ambient pressure conditions together with its mother liquor into the DAC (Figure 3.2.a-c). Subsequent experiments focused on loading crystals of the complex and using demineralised water as crystallisation medium (Figure 3.2.d-f).

The in situ dissolution process is also dependent on crystal size: although temperature was cycled using a heatgun up to $373 \mathrm{~K}$, incomplete dissolution was achieved if the DAC contained too much crystalline material. Although considerable time was spent in order to vary and optimise the crystallisation conditions, cycling the pressure in the range of $0.1 \mathrm{MPa}-0.72 \mathrm{GPa}$ did not initiate the recrystallisation process. Reducing the temperature of the DAC to $277 \mathrm{~K}$ was also not helpful. Overall, high-pressure techniques did not lead to crystallisation of any crystalline form of $\alpha-\mathrm{CD}$ or SA.
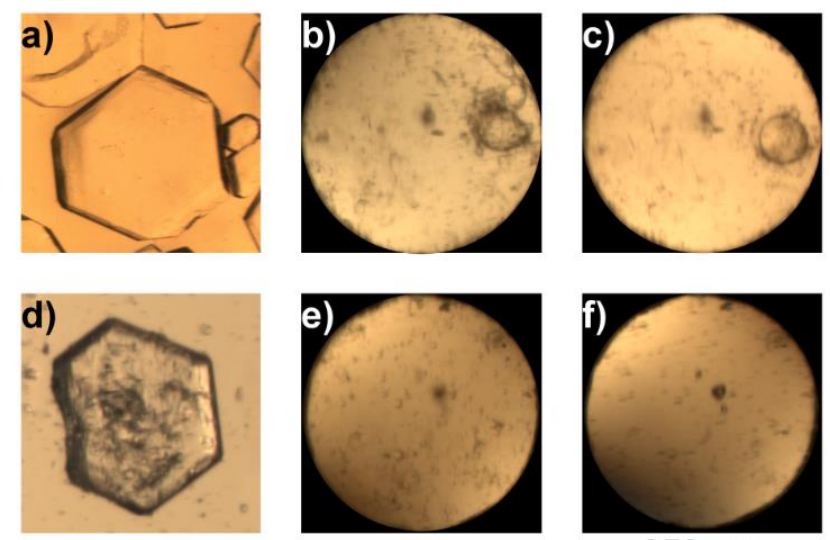

$350 \mu \mathrm{m}$

Figure 3.2. High-pressure crystallisation attempts of $\alpha$-CD·SA inclusion complex. (a) Single crystal of $\alpha$ CD.SA inclusion complex in its mother liquor; (b) and (c) Mother liquor used as crystallisation medium: (b) Incomplete dissolution of the single crystal at $0.17 \mathrm{GPa}$, (c) Dissolution remains incomplete at $0.49 \mathrm{GPa}$ and 393 K; (d) Single crystal out of the mother liquor; (e) and (f) Demineralised water used as crystallisation medium: (e) Complete dissolution at $0.17 \mathrm{GPa}$; (f) No crystallisation on compression to $0.72 \mathrm{GPa}$. 


\subsubsection{Data collection and reduction}

Several crystals were tested on the diffractometer. Although the crystals did not show cracks under the microscope when left out of the mother liquor (Figure 3.2.d), they were found to be unstable at room-temperature conditions, yielding poor diffraction quality even at low temperature. Maintaining low temperature from the moment the crystal was extracted from the mother liquor until final mounting on a glass fibre on the goniometer's head was essential to have good diffraction quality: in order to achieve this, a drop containing mother liquor and crystals was pipetted onto a microscope slide that had previously been cooled using an ice bath beneath the slide. Subsequently, the crystal was moved from the mother liquor to a cold drop of mounting oil and then to the goniometer's head under a cold and dry nitrogen stream. ${ }^{17}$

Data collection was undertaken using a Bruker-AXS APEX II diffractometer equipped with graphite-monochromatic Mo-K $\alpha$ and an Oxford Cryosystems low-temperature device. Data integration and global-cell refinement were performed with the program SAINT.18 Absorption correction was performed with $S A D A B S .{ }^{19}$ Structure solution of this medium-sized structure was based on Patterson-seeded dual-space recycling in the SHELXD program..$^{20}$ Structures were refined by full-matrix least squares against $F^{2}$ using SHELXL-2014/721 through the SHELXLE GUI..$^{22}$ Stereochemical restraints for the host and guest structures were applied to increase the robustness of the refinement: restraints were generated by the GRADE program using the GRADE Web server. ${ }^{23}$ A GRADE dictionary for SHELXL contains target values and standard deviations for 1,2distances (DFIX) and 1,3-distances (DANG), as well as restraints for planar groups (FLAT). Anisotropic displacement parameters were refined using the new rigid bond restraint (RIGU) implemented in the SHELXL2014 program. ${ }^{24}$ All H-atoms of $\alpha$-CD and SA were placed geometrically and allowed to ride on the parent atoms. H-atoms belonging to ordered water molecules were clearly visible in difference Fourier maps and their positions were refined subject to distance restraints. $U_{\text {iso }}(\mathrm{H})$ values were assigned in the range 1.2-1.5 times $U_{\text {eq }}$ of the parent atom. Details on the treatment of disorder are detailed in the discussion below and crystallographic details can be found in Table 3.1.

\subsection{Results \& discussion}

Mixing an equimolar ratio of $\alpha$-CD with $\mathrm{SA}$ in water, and using low temperature conditions, led to the crystallisation of hexagonal prism shaped crystals. A first microscopic analysis of the crystals through the hexagonal face (Figure 3.2.a Figure 3.2.d) by using polarised light showed an absence of light extinction: this is an indication of two crystallographically equivalent directions being perpendicular to the polarised light $(a=b)$, a characteristic of uniaxial 
crystals; in this case the $a b$ plane corresponds to the hexagonal face of the crystal. The crystal thus belongs to trigonal, tetragonal, or hexagonal crystal system.

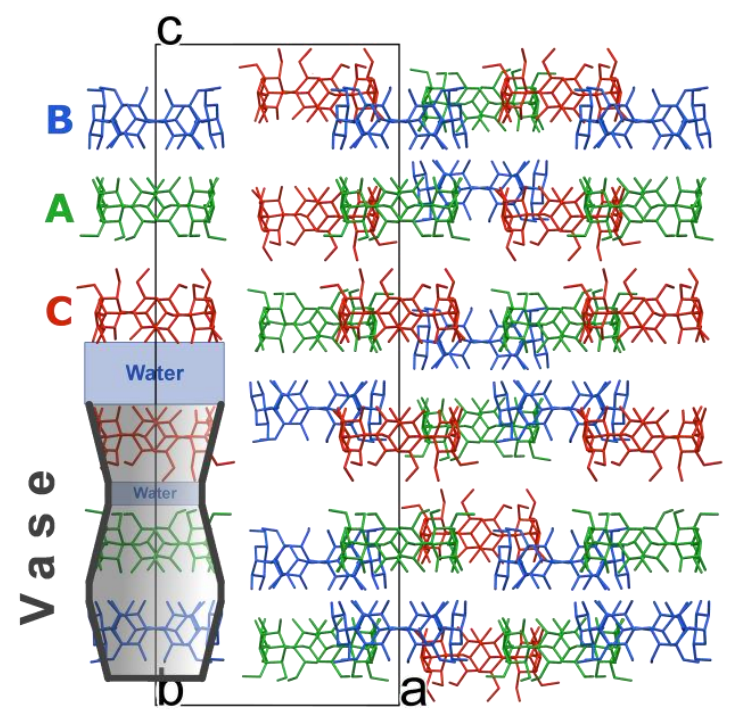

Figure 3.3. Crystal packing of $\alpha$-CD molecules viewed along the $b$-axis. H atoms, disorder of $\alpha$-CD C, SA and water molecules have been omitted for clarity. Symmetry-equivalent molecules are colour-coded.

This microscopic analysis endorsed the choice of the unit cell from X-ray diffraction, with successful indexing of the reflections using a rhombohedral unit cell. The reflections could also be indexed using a lower symmetry unit cell, through the transformation matrix:

$$
\left(\begin{array}{ccc}
-1 / 3 & -2 / 3 & -2 / 3 \\
1 & 0 & 0 \\
-1 / 3 & -2 / 3 & 1 / 3
\end{array}\right)
$$

The choice of a rhombohedral crystal system dictates that both host and guest molecules sit on a 3-fold rotation axis going through $\alpha$-CD cavities. Heavy disorder of the guest, evident from the electron density maps, could be modelled in the higher symmetry space group and this was finally chosen for refinement.

The inclusion complex crystallises in rhombohedral space group $R 32$ with $3 \times 1 / 3 \alpha$-CD molecules in the asymmetric unit, here named A, B and C (Figure 3.3). Each $\alpha$-CD encloses one SA molecule disordered over six positions, in other words there are two SA molecules, with $1 / 6$ site occupancy each, per $\alpha$-CD cavity disordered over the 3-fold rotation axis, overall giving an $\alpha$ CD.SA 1:1 complex. $\alpha$-CD C is further disordered over two positions with $2 / 3(\mathrm{C})$ and $1 / 3\left(\mathrm{C}^{\prime}\right)$ site occupancies. In addition, the unit cell contains ca. 12 water molecules making the complex a pseudo dodecahydrate. 


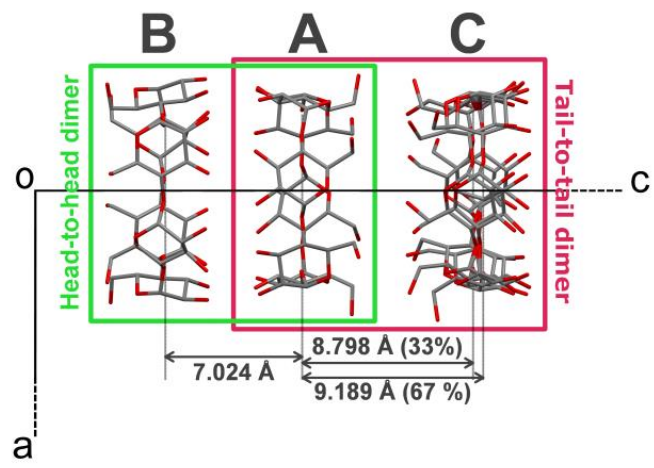

Figure 3.4. Projection of $\alpha$-CD·SA structure along the $b$-axis. H atoms, SA and water molecules have been omitted for clarity. The distances refer to gap in Å between the least-square planes formed by all O4 atoms involved in the glycosidic bonds.

$\alpha$ - and $\beta$-CD molecules in the solid state have always been known, to the best of our knowledge, to pack as distinct entities or dimers. ${ }^{13,25}$ In this work, a new building block is observed: $\alpha$-CD molecules form trimers packed along the $c$-axis. Each trimer is made of crystallographically independent molecules assembled in a stacked vase-like cluster (Figure 3.3). The stacked trimer is not unknown for $\gamma$-CD molecules (see for example CSD refcodes FEJFIJ, FEJFOP, NUNRIX, SIBJAO, SIBJES);26-28 however the concept of vase-like packing has not been previously reported. It has also been reported that $\beta$-CD molecules crystallise as trimers (CSD refcodes RIPKIL, OCIGAK)29,30 or tetramers. ${ }^{31,32}$ The structure of the title compound appears to be very similar to that of $\alpha$-CD.hexaethylene glycol reported by Harada et al. (CSD refcode LOJTUZ, no 3D coordinates deposited in the $(\mathrm{CSD})^{33}$; however, this thesis neither describe the distinctive trimer arrangement in detail, nor identifies a new packing type. In contrast, in a conference abstract, Caira et al. have recently reported that the $\alpha$-CD-lipoic acid system "crystallizes in the trigonal system, space group R32, with three independent $C D$ molecules in the asymmetric unit and is not isostructural with any known $C D$ complex" ${ }^{\prime \prime}$. These observations make the reported packing type rare but not unknown.

The vase-like cluster is formed by two sub-dimers: 1) a head-to-head dimer, which is stabilised by H-bonds between secondary hydroxy groups of $\alpha$-CDs A and $\mathrm{B}$, and 2) a tail-to-tail dimer between $\alpha$-CDs A and $\mathrm{C}$ interconnected through a cluster of water molecules. Analysis of the glycosidic planes of CD molecules shows that the distance between these planes in the head-tohead dimer is $7.024 \AA$, compared to a mean distance of $9.05997 \AA$ for the tail-to-tail dimer (

Figure 3.4). Successive vase-like clusters, stacked via 2 -fold rotation symmetry, are separated by a layer of water molecules forming a complex H-bonded network which holds two vase-like structures together.

The three $\alpha$-CD molecules forming the vase-like cluster are parallel and exhibit an almost ideal cylindrical shape. They form infinite linear channels extending along the c-axis of the unit 
cell. These channels are forming a honeycomb packing type where each $\alpha$-CD molecule is surrounded by a hexagonal layer of six neighbouring $\alpha$-CD molecules (Figure 3.5). A honeycomb or quasi-hexagonal pattern is typical for effective close packing, ${ }^{28,34}$ unlike the square or rectangular patterns, and it was reported for the first time by Saenger in 1980, while describing the general arrangement of CDs in crystals, as "hexagonal packing of stacks". ${ }^{35}$ Although a careful analysis of the reported channel type structures of $\alpha-\mathrm{CD}$ inclusion complexes, including hydrates, in the CSD shows that $\alpha$-CD molecules exhibit the honeycomb packing type in $27^{10,36-56}$ out of 50 structures (CSD refcodes of these structures are reported in the Appendix 3.7), the hexagonal packing type has been rarely reported. ${ }^{37,40,48}$ Instead, authors usually report the type of $\alpha$-CD dimer arrangement, whether it is head-to-head or head-to-tail, in channel-type structures. ${ }^{10,36-52}$ Harada et al. are the only authors naming the hexagonal arrangement as honeycomb for $\alpha$-CD.hexa-ethylene glycol, a highly similar structure to the structure of the title compound (CSD refcode LOJTUZ, no 3D coordinates deposited in the CSD). ${ }^{33}$

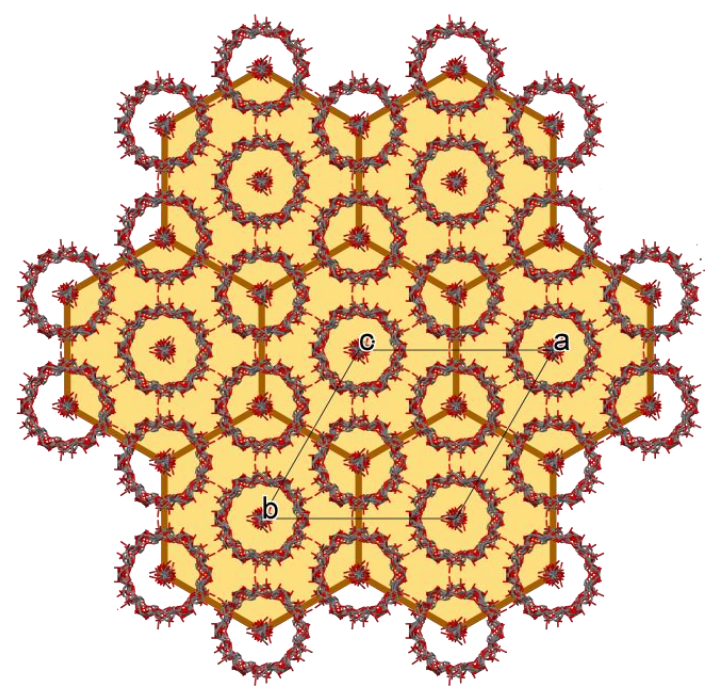

Figure 3.5. The honeycomb packing mode of $\alpha$-CD.SA structure along the $c$-axis. $\mathrm{H}$ atoms and water molecules have been omitted for clarity.

One of the features of $\mathrm{CDs}$ is the flexibility of the primary-hydroxy groups. The conformation of the hydroxy group is defined by the value of the $\mathrm{O}(5)-\mathrm{C}(5)-\mathrm{C}(6)-\mathrm{O}(6)$ torsion angle: a preferred (-)-gauche conformation in which the hydroxy group is facing the exterior of the cavity, and a less preferred (+)-gauche conformation where the hydroxy group is facing the inner cavity. ${ }^{57}$ All but one primary hydroxy groups in $\alpha$-CD C exhibit the (-)-gauche conformation and are directed away from the cavity. The rotation of the primary hydroxy group to the less favoured (+)-gauche conformation can be explained by the formation of the short H-bonds [2.53(4) and 2.79(1) ̊] with a disordered water molecule. A CSD (V 5.36) search shows that (-)-gauche conformers are not unknown for $\alpha$-CD inclusion complexes and indicate the absence of direct $\mathrm{H}$ - 
bonds between the guest and the host molecules, which could explain the high degree of rotational disorder of the guest observed here. In contrast, in the structure of $\beta$-CD.SA which contains one full guest and host molecules in the asymmetric unit, SA is H-bonded to two primary CD hydroxy groups, which are facing the inner cavity, through one water molecule each. Interestingly, the SA molecule in the $\beta$-CD.SA inclusion complex does not exhibit disorder.

A search in the CSD (V 5.36) based on the $C(1)-C(2)-C(3)-C(4)$ torsion angle of the guest (Figure 3.1.a) shows that SA molecules exhibit two conformations in the solid state, trans and gauche, with an incidence of 90 and $10 \%$, respectively, out of a total of 165 structures. Lisnyak et al. reported that the trans conformer is energetically more favourable than the gauche one with an energy difference of $31.4 \mathrm{~kJ} \cdot \mathrm{mol}^{-1}{ }^{6}$ Whilst SA molecules exhibit the gauche conformer inside $\beta$-CD cavity (CSD refcode: KIJSEC), ${ }^{6}$ the electron density maps of $\alpha$-CD.SA point to the trans conformation with SA molecules extended along the $c$-axis. Thanks to the extended conformation, SA and water molecules form infinite $\mathrm{H}$-bonded chains embedded inside the vase-like clusters (Figure 3.6). In the B-A head-to-head dimer, both SA molecules are directly H-bonded to each other through the carboxylic groups while in the A-C tail-to-tail dimer SA molecules are linked via $\mathrm{H}$ bonding to water. The planes formed by the glycosidic O-atoms of the ordered CD molecules A and B in the head-to-head dimer are almost orthogonal to the planes formed by the SA molecules inside the respective cavities [SA planes were calculated using $\mathrm{C}(1), \mathrm{C}(2), \mathrm{C}(3), \mathrm{C}(4)$ ]. For $\alpha$-CD $\mathrm{C}$, similar calculations show that the same planes are 13 to $18^{\circ}$ off from being orthogonal.

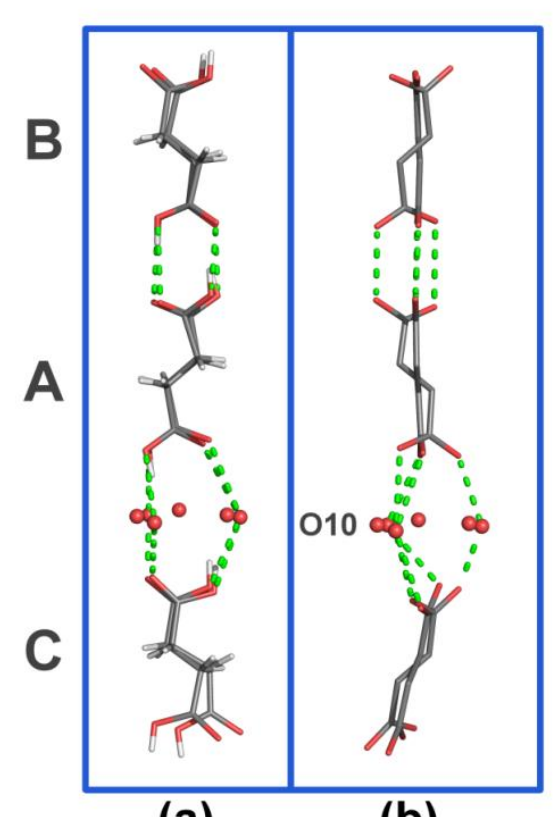

(a)

(b)

Figure 3.6. H-bonded motif formed by SA molecules in the asymmetric unit viewed a) along the $a$-axis and b) along the $b$-axis. . O $\cdots \mathrm{O}$ contacts are represented by dashed green lines. $\mathrm{H}$-atoms (in $\mathrm{b}$ ), $\alpha$-CD and water molecules have been omitted for clarity. 


\subsubsection{Modelling of disorder}

The structural refinement of $\alpha$-CD.SA was particularly challenging due to the heavy disorder of the host, guest and solvent molecules. The disordered $\alpha$-CD C molecule was modelled using a free variable fraction which refines to a final value of $0.673(5)$ for the major component. The effect of disorder is to elongate the vase-like cluster (Figure 3.3). At $90 \mathrm{~K}$, a careful inspection of the difference Fourier maps permits the refinement of 33 positions of water molecules which are distributed heterogeneously over the asymmetric unit and of which only two are fully occupied. For these two water molecules, H-atoms could be located in difference Fourier maps.

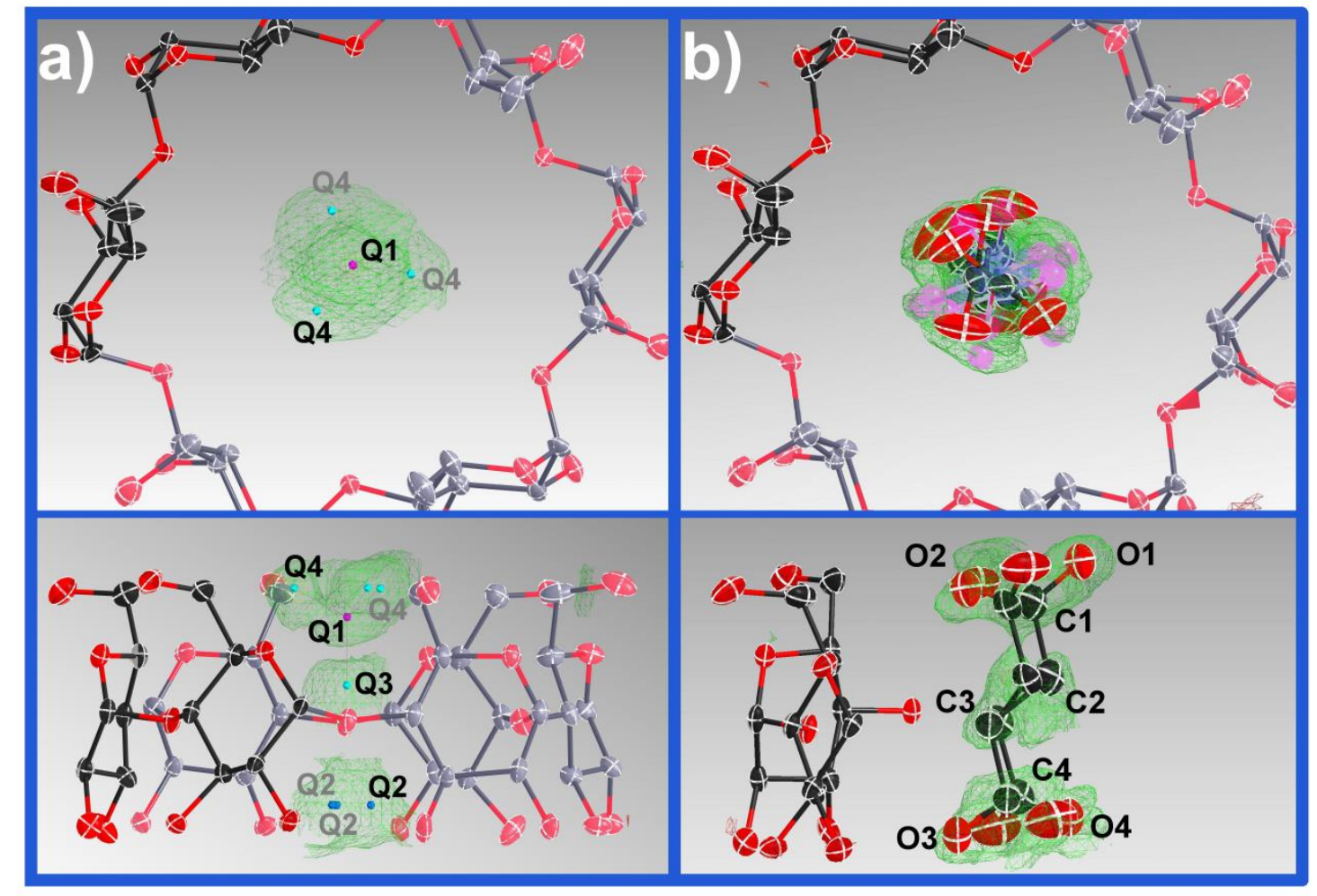

Figure 3.7. Fobs-Fcalc maps in green showing the electron density before (a) and after (b) modelling SA inside $\alpha$-CD B molecule. The peaks Q1 and $3 \times(\mathrm{Q} 4)$ are forming a tetrahedron. $\mathrm{H}$-atoms and the rest of the structure have been omitted for clarity. Displacement ellipsoids are drawn at the $50 \%$ probability level.

The channel-type structure of $\alpha$-CD·SA favours the guest to be in the trans, planar conformation. The 3 -fold rotation axis, going through the centre of the host, is incompatible with the guest molecular symmetry and imposes the presence of disorder, which was modelled as exemplified by $\alpha$-CD molecules B (Figure 3.7) as follows: electron density maps inside the $\alpha$-CD cavity show tetrahedral features, incompatible with a single SA molecule. The difference electron density peak $\mathrm{Q}(1)$, sitting on the 3-fold rotation axis, was assigned to the carbon atom $\mathrm{C}(1)$ of the carboxylic group. The coordinates of $\mathrm{C}(1)$ were constrained to make model building more straightforward. By using stereochemical restrains generated with the GRADE webserver ${ }^{23}$ all other $\mathrm{Q}$ peaks were correctly assigned, i.e. $\mathrm{Q}(4)$ to $\mathrm{O}(1)$ and $\mathrm{O}(2), \mathrm{Q} 3$ to $\mathrm{C}(2), \mathrm{Q}(2)$ to $\mathrm{C}(4)$..etc (Figure 3.7). Finally, all atomic coordinates were freely refined. Two SA molecules fit inside the 
disordered electron density; the negative PART instruction was used in the program SHELXL to exclude the generation of special position constraints and dissociate bonding to symmetry generated atoms within the same PART group.

After several trials, the best refinement strategy consisted of gradually modelling the electron density of the host molecules and the reliably localised solvent peaks, followed by the guests, and finally the rest of the disordered solvent molecules. In order to avoid overparametrisation, site-occupancy factors of the disordered water molecules were fixed to the initially refined values. Those of the guest molecules were constrained to $1 / 6$ each. H-atoms belonging to disordered water molecules were not placed during refinement but were taken into account for the calculation of $F_{000}$ and derived properties.

Due to the limited resolution and structural complexity of the model, soft restraints on all bond lengths and angles were applied using the GRADE webserver. ${ }^{23}$ Following a CSD search on structures containing SA-SA H-bonded dimer, SA molecules were restrained to be planar.58-62 Restraints for both SA and $\alpha$-CD were also applied on the atomic displacement parameters by using SIMU, DELU and RIGU SHELXL instructions. ${ }^{24}$ ISOR restrains were used on a handful of atoms to cure ill-defined ADPs.

\subsubsection{Insights into the disorder:}

In an attempt to gain a better insight into the disorder of $\alpha$-CD.SA inclusion complex and to investigate whether a temperature-dependent phase transition takes place, we extended our initial work to a multi-temperature investigation covering the $100-270 \mathrm{~K}$ temperature range, in an ascending temperature ramp.

The crystallographic data of all structures summarised in Table 3.1 show small differences in the lattice constants across the temperature range. The lattice parameters were normalised to the unit cell values of the $100 \mathrm{~K}$ crystal structure and are visually represented in Figure 3.8. They describe thermal expansion up to $180 \mathrm{~K}$ for both the $c$ - and $a$-axes. For the unit cell volume, the thermal expansion is approximately linear and isotropic up to this temperature. From $180 \mathrm{~K}$ to 240 $\mathrm{K}$, the complex overall expands but the $c$-axis shows negative thermal expansion. The negative thermal expansion for this axis is very marked between 240 and $270 \mathrm{~K}$ so that a slight volume contraction is concomitantly observed.

These variations in the unit-cell constants can be related to the following structural features: 1) the isotropic and linear expansion of the $a$-axis $(a=b)$ is associated with the expansion of the intermolecular contacts between adjacent $\alpha$-CD molecules along the same axes; 2 ) the negative thermal expansion along the c-axis is associated with a shortening of the vase-like cluster (Figure 
3.9): the small water layer stabilising the $\alpha$-CD A-C tail-to-tail dimer (Figure 3.3) compresses as function of increasing temperature causing a shortening of the $\alpha$-CD A-C dimer.

The small water layer is formed by one disordered water molecule [named O(10)_91 in the structure] $\mathrm{H}$-bonded to the (+)-gauche, above mentioned, primary hydroxy group of $\alpha$-CD C molecule. The distance between the $\mathrm{O}(6)$-atom of this hydroxy group and the water molecule increases from 2.80(2) to 3.01(5) $\AA$, at 100 and $270 \mathrm{~K}$, respectively. Ordering of $\alpha$-CD C is observed at $270 \mathrm{~K}$ although the structure has large temperature factors. This is in fact an indication of dynamic disorder where low temperatures freeze the movement of $\alpha$ - $\mathrm{CD} C$ into two distinct positions, and at higher temperatures $\alpha$-CD C vibrates along the $c$-axis fitting only one position in the electron density map. Ordering is associated with a rearrangement of hydroxy groups: $\alpha$-CD C has two (+)-gauche primary hydroxy conformers, instead of one observed at low temperature. The new (+)-gauche conformer, pointing towards the inner cavity, is H-bonded to the disordered water molecule O(10)_91 with a distance of 2.64(9) $\AA$. This indicates that there is interplay between movement of $\alpha-\mathrm{CD}$ and water molecules towards stabilisation of the structure. These small movements generate overall changes in the structure seen along the $c$-axis (See appendix 3.7, Figure 3.27).

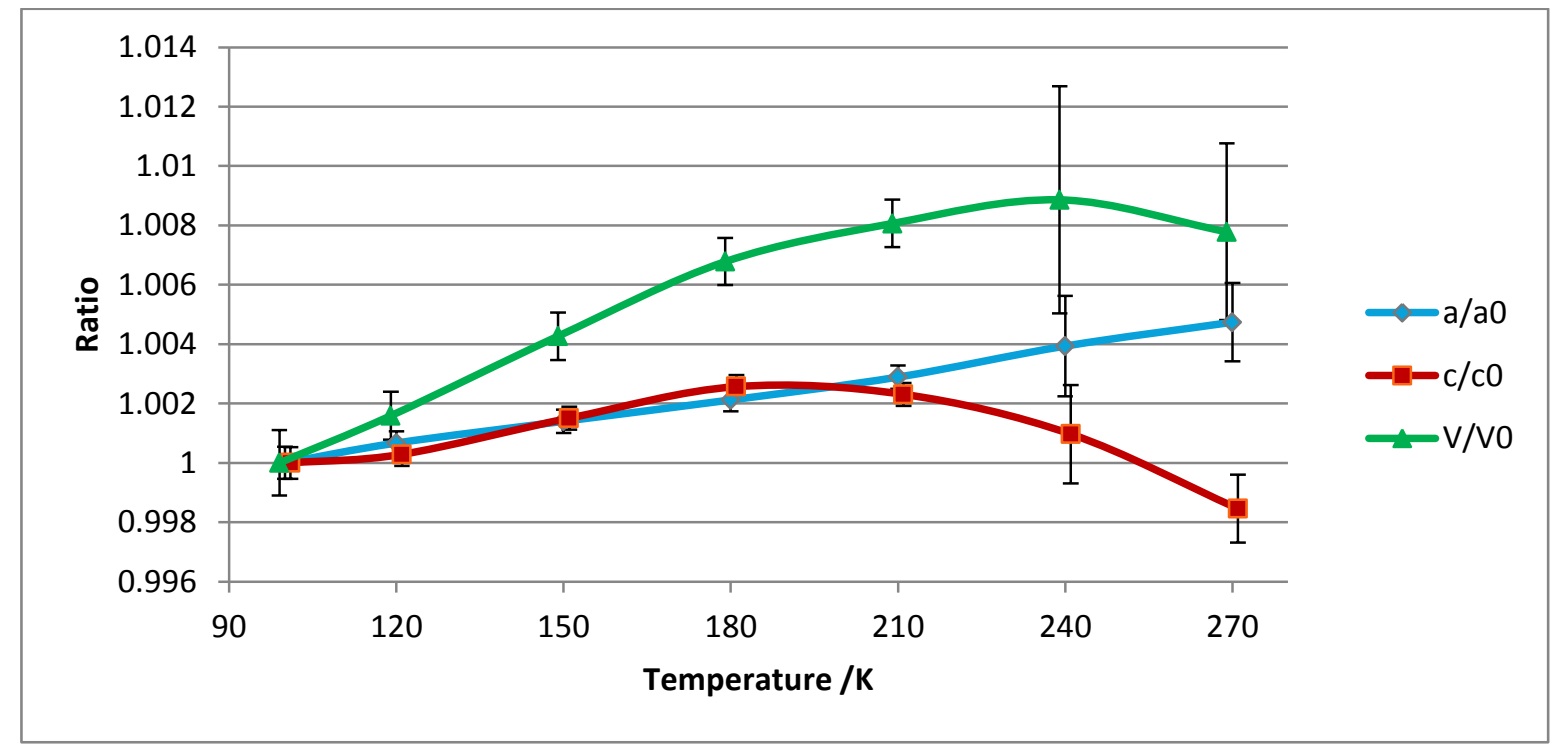

Figure 3.8. The evolution of the normalised unit cell parameters as function of temperature.

Errors are at $3 \sigma$ level. Details are available in the appendix 3.7. 


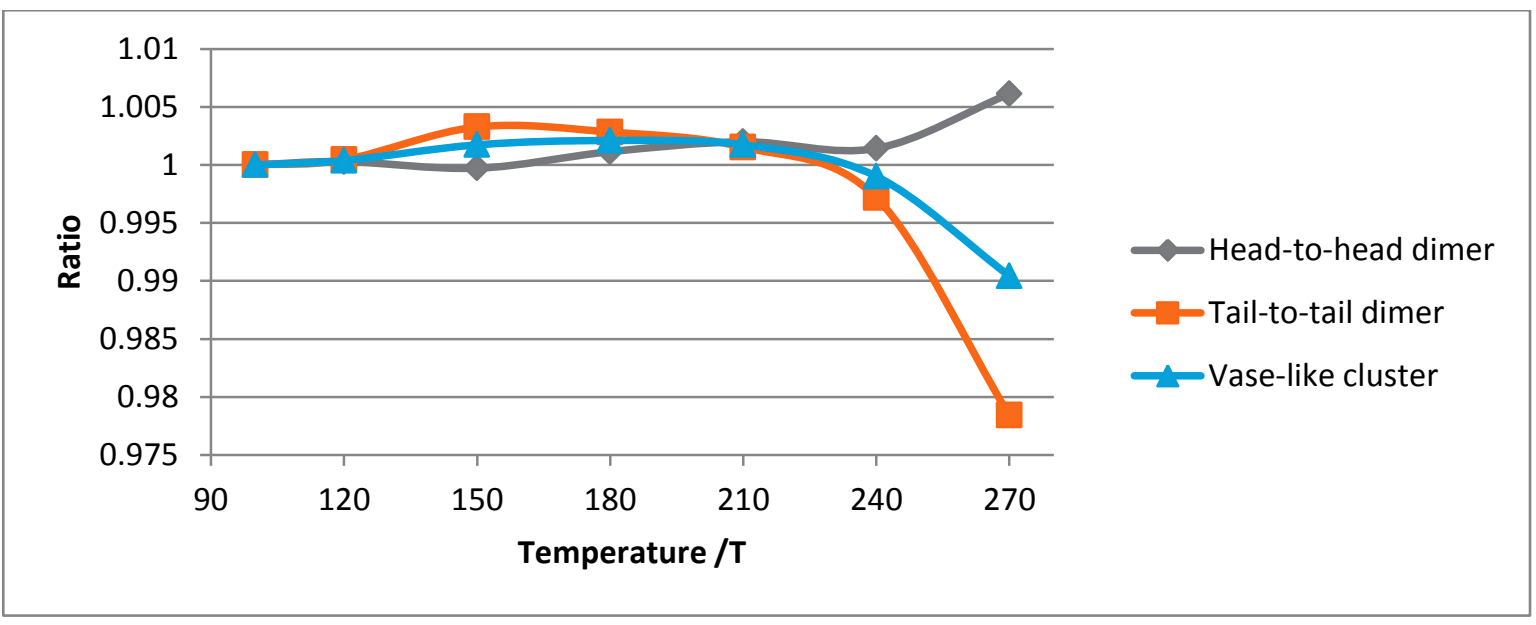

Figure 3.9. Evolution of the normalised distances between dimers in $\alpha$-CD.SA.

Distances were calculated based on the $\mathrm{O}(4)$ glycosidic planes using MERCURY. 63

The $100 \mathrm{~K}$ structural model was refined based on the atomic coordinates of the $90 \mathrm{~K}$ model, and was then used as a starting model for all other temperature points. Modelling of disordered water molecules in a highly hydrated structure is subject to user bias. Temperature factors and site occupancy parameters are correlated and in the absence of independent information, e.g. on water content determined by thermal analysis, the user must make judicious use of refinement tools in order to obtain a reasonable model, which does not necessarily lead to the lowest $R$-factor. Comparing Fourier and difference Fourier maps can give an indication of the mean position and site occupancy of the atoms provided a consistent data reduction and refinement strategy for all data points are applied. To minimise model bias and double check the stability of the model, the SHELXL WIGL command was used as a cross-validation method during the refinement. ${ }^{64}$ Two sets of results are reported. In the first set, all water molecules were placed and refined according to the difference Fourier electron density maps (results in Table 3.1). In the second set, all water molecules were omitted during refinement in order to compare the evolution of the maps as a function of increasing temperature. The latter set of structures can be used to emphasise the change in the positions of water molecules and site occupancies by two methods described as follows: 
Table 3.1: Crystallographic data of the multi-temperature structures of $\alpha$-CD·SA inclusion complex

\begin{tabular}{|c|c|c|c|c|c|c|c|c|}
\hline & Crystal 1 & & & & Crystal 2 & & & \\
\hline$T / K^{\mathrm{a}}$ & 90(2) & $100(2)$ & $120(2)$ & $150(2)$ & $180(2)$ & $210(2)$ & $240(2)$ & $270(2)$ \\
\hline Formula & $\begin{array}{c}\mathrm{C}_{36} \mathrm{H}_{60} \mathrm{O}_{30} \\
\mathrm{C}_{4} \mathrm{H}_{6} \mathrm{O}_{4} \\
11.83\left(\mathrm{H}_{2} \mathrm{O}\right)\end{array}$ & $\begin{array}{c}\mathrm{C}_{36} \mathrm{H}_{60} \mathrm{O}_{30}, \mathrm{C}_{4} \mathrm{H}_{6} \mathrm{O}_{4} \\
12.14\left(\mathrm{H}_{2} \mathrm{O}\right)\end{array}$ & $\begin{array}{c}\mathrm{C}_{36} \mathrm{H}_{60} \mathrm{O}_{30}, \mathrm{C}_{4} \mathrm{H}_{6} \mathrm{O}_{4} \\
12.23\left(\mathrm{H}_{2} \mathrm{O}\right)\end{array}$ & $\begin{array}{c}\mathrm{C}_{36} \mathrm{H}_{60} \mathrm{O}_{30}, \mathrm{C}_{4} \mathrm{H}_{6} \mathrm{O}_{4} \\
11.54\left(\mathrm{H}_{2} \mathrm{O}\right)\end{array}$ & $\begin{array}{c}\mathrm{C}_{36} \mathrm{H}_{60} \mathrm{O}_{30}, \mathrm{C}_{4} \mathrm{H}_{6} \mathrm{O}_{4} \\
10.86\left(\mathrm{H}_{2} \mathrm{O}\right)\end{array}$ & $\begin{array}{c}\mathrm{C}_{36} \mathrm{H}_{60} \mathrm{O}_{30}, \mathrm{C}_{4} \mathrm{H}_{6} \mathrm{O}_{4} \\
10.27\left(\mathrm{H}_{2} \mathrm{O}\right)\end{array}$ & $\begin{array}{c}\mathrm{C}_{36} \mathrm{H}_{60} \mathrm{O}_{30}, \mathrm{C}_{4} \mathrm{H}_{6} \mathrm{O}_{4} \\
9.29\left(\mathrm{H}_{2} \mathrm{O}\right)\end{array}$ & $\begin{array}{c}\mathrm{C}_{36} \mathrm{H}_{60} \mathrm{O}_{30}, \mathrm{C}_{4} \mathrm{H}_{6} \mathrm{O}_{4} \\
7.97\left(\mathrm{H}_{2} \mathrm{O}\right)\end{array}$ \\
\hline Crystal system & Trigonal & & & & Trigonal & & & \\
\hline Space group & R $32: H$ & & & & R $32: H$ & & & \\
\hline$a=b / \AA$ & $23.6625(6)$ & $23.645(3)$ & $23.6608(7)$ & $23.6781(6)$ & $23.6951(5)$ & 23.7133(6) & 23.738(13) & 23.757(10) \\
\hline$c / \AA ̊ \AA$ & $55.7235(15)$ & $55.706(7)$ & $55.722(2)$ & $55.7898(18)$ & $55.8490(15)$ & $55.8345(17)$ & $55.76(3)$ & $55.62(2)$ \\
\hline$\alpha=\beta / /^{\circ}$ & 90 & & & & 90 & & & \\
\hline$\gamma /{ }^{\circ}$ & 120 & & & & 120 & & & \\
\hline$V / \AA^{3}$ & 27020.3(16) ^̊3 & 26973(7) $\mathrm{A} 3$ & 27015.8(19) ^̊3 & 27088.1(16) ^̊3 & 27155.8(14) ̊̊3 & 27190.5(16) А̊3 & 27212(34) ̊3 & 27183(26) $\AA 3$ \\
\hline $\mathrm{Z}$ & 18 & & & & 18 & & & \\
\hline$Z^{\prime}$ & 1 & & & & 1 & & & \\
\hline$\left.D_{\text {calc }} / \mathrm{g} \mathrm{cm}^{-3}\right)$ & 1.448 & 1.451 & 1.451 & 1.433 & 1.416 & 1.403 & 1.383 & 1.357 \\
\hline $\begin{array}{l}\text { Meas. / uniq. / obs. } \\
\text { Reflections }^{\mathrm{b}}\end{array}$ & $\begin{array}{c}118804 / 11028 \\
/ 9148\end{array}$ & 45399 / 6390 / 5109 & 46751 / 6302 / 4512 & 47260 / 6315 / 4645 & 47564 / 6328 / 5099 & 47801 / 6347 / 5061 & $21178^{*} / 6034$ / 3799 & $29882^{*} / 6298$ / 3592 \\
\hline$d_{\max } / \AA ̊$ & 0.83 & 1.00 & 1.00 & 1.00 & 1.00 & 1.00 & 1.00 & 1.00 \\
\hline Completeness to $d_{\max }$ & 0.999 & 0.991 & 0.977 & 0.974 & 0.974 & 0.976 & 0.969 & 0.974 \\
\hline$R_{\text {int }}$ & 0.0641 & 0.0752 & 0.1074 & 0.0988 & 0.0885 & 0.0877 & 0.0908 & 0.1253 \\
\hline $\begin{array}{c}\text { Data/restraints/ } \\
\text { parameters }\end{array}$ & $\begin{array}{c}11028 / 4303 / 13 \\
19\end{array}$ & $6390 / 4279 / 1247$ & $6302 / 4284 / 1211$ & $6315 / 4257 / 1201$ & $6328 / 4209 / 1225$ & $6347 / 3267 / 1147$ & $6034 / 3263 / 1110$ & $6298 / 2079 / 903$ \\
\hline$R_{1}{ }^{c}$ & 0.0470 & 0.0487 & 0.0573 & 0.0561 & 0.0691 & 0.0717 & 0.0724 & 0.0826 \\
\hline$w R_{2}^{d}$ & 0.1142 & 0.1046 & 0.1180 & 0.1191 & 0.1523 & 0.1649 & 0.1718 & 0.2041 \\
\hline
\end{tabular}

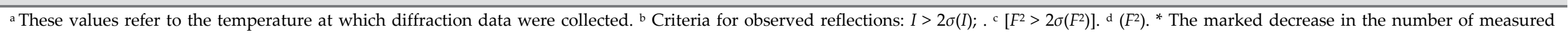
reflections at $240 \mathrm{~K}$ and $270 \mathrm{~K}$ is explained by a slight movement of the single crystal on the goniometer head at $240 \mathrm{~K}$. 
a) An analysis with SHELXLE GUI22 allows a qualitative analysis of the changes in the difference density as a function of temperature. Figure 3.10 clearly illustrates the loss of this signal from the solvent region as temperature is increased.

b) Void analysis performed with Mercury ${ }^{63}$ using a probe radius of $1.2 \AA$ and a grid spacing of $0.2 \AA$, calculated using the contact surface algorithm, reveals that the volume occupied by the solvent does not change significantly as temperature is increased: water molecules occupy a mean volume of 19.4(2)\% of the total unit cell volume (Figure 12). $\alpha$-CD C' was not taken into account for void analysis.

A third method would consist of using The SQUEEZE ${ }^{65}$ algorithm implemented in the PLATON program which also gives an indication of the solvent accessible volume. ${ }^{66,67}$ The SQUEEZE routine is typically used to obtain the electron count in a disordered region and hence aid in the assignment of type and number of disordered solvent molecules. However, to obtain meaningful electron count numbers, the data should be of sufficient resolution and, more importantly, all low order reflections should be measured. Since this was not the case in this study, details of electron count as well as the solvent accessible volume have been omitted here.

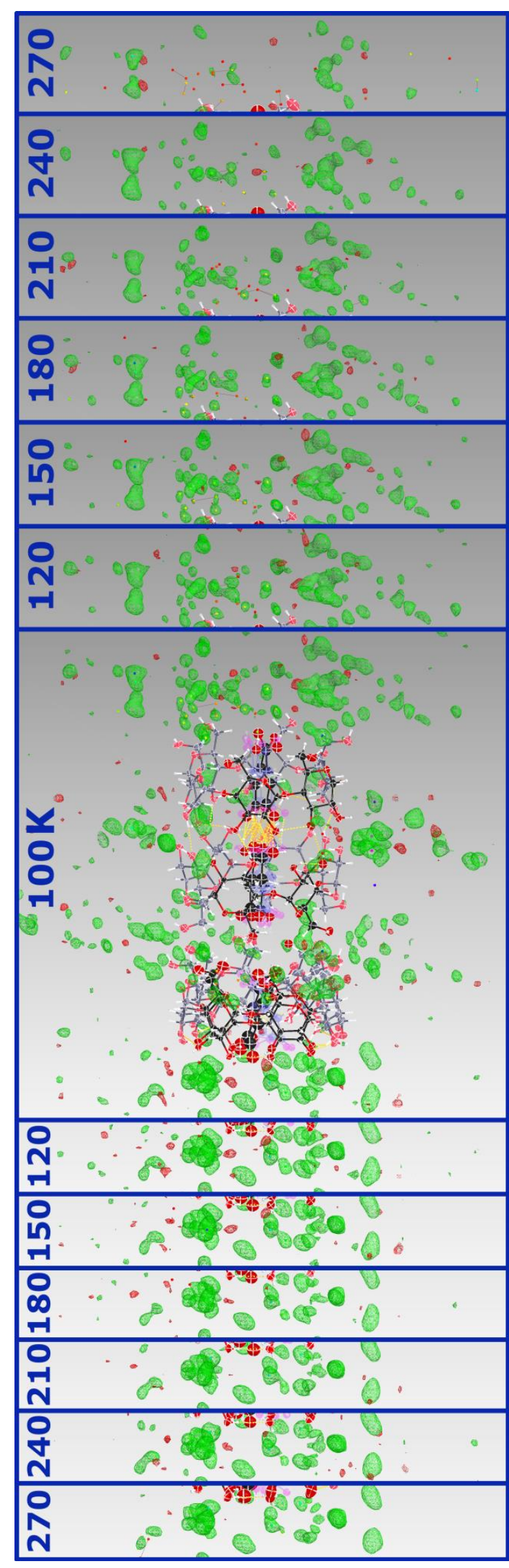

Figure 3.10. SHELXLE Fobs-Falc maps of $\alpha$-CD.SA complex. SHELXLE Fobs- $F_{\text {calc }}$ maps (at $0.40 \mathrm{e}^{-} / \AA^{3}$ ) of $\alpha$ CD.SA inclusion complex at $100 \mathrm{~K}$ and the respective solvent regions on both ends of the vase-like cluster at the different temperatures showing the decrease of the signal as a function of increasing temperature. 


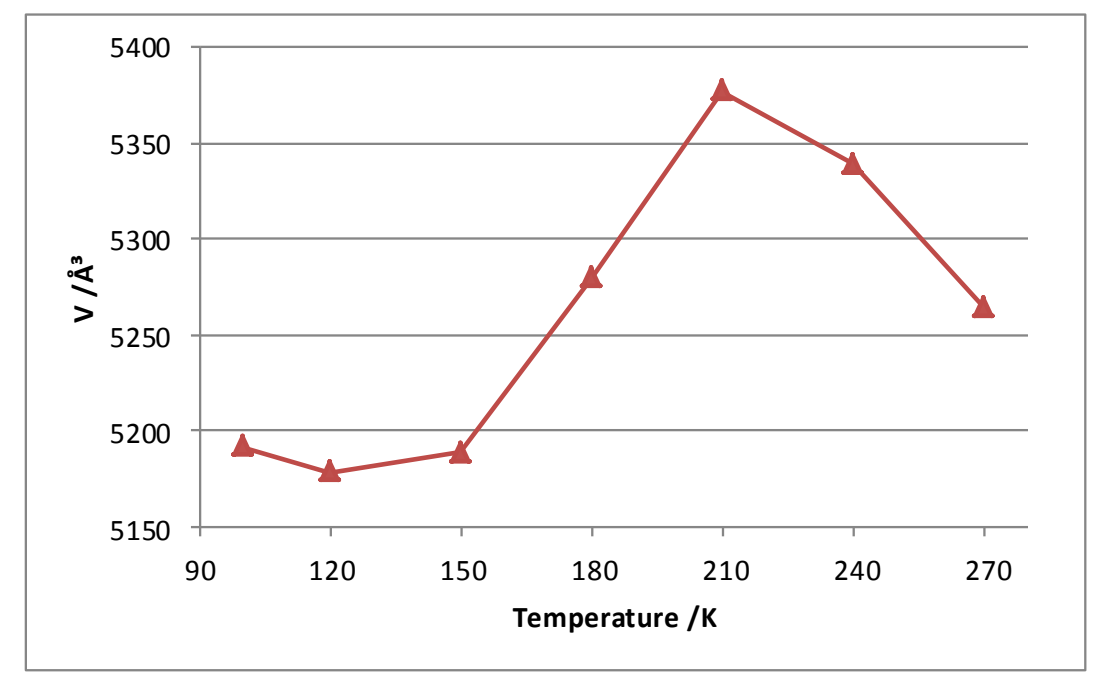

Figure 3.11. The solvent accessible void volume calculated using MERCURY ${ }^{63}$ (grid spacing $=0.2 \AA$; probe radius $=1.2 \AA$ ).

An analysis, based on the fully refined structures, of the anisotropic displacement parameters (ADP) as function of temperature indicates larger thermal motion at higher temperatures, as expected. (See Appendix Figure 3.12-26). Analysis of the refined $\mathrm{U}_{\mathrm{ij}}$ values of $\mathrm{O}(1) \rightarrow \mathrm{O}(6)$ water molecules, which are located in the space between adjacent $\alpha$-CD columns (Figure 3.3), show comparable expansion of the three diagonal elements $U_{11}, U_{22}, U_{33}$ of the ADP tensor. These diagonal elements are parallel to the reciprocal unit-cell axes $a^{*}, b^{*}$ and $c^{*}$, respectively, and in the case of a rhombohedral crystal system, $\mathrm{U}_{33}$ is parallel to the $c$-axis. For $\alpha$-CD atoms, the expansion of $\mathrm{U}_{33}$ is much more pronounced compared to the two other directions. Hence, while water molecules increase their vibrations in all directions as temperature increases, $\alpha$-CD molecules have more freedom along the $c$-direction.

To summarise, the loss of diffraction quality at higher temperatures can be directly ascribed to structural disorder, primarily of the solvent region. It is not unlikely that at even higher temperatures, water molecules vibrate excessively leading to disruption of the crystal packing and structural instability as testified by complete loss of diffraction at 298 K. Given the channel-type packing adopted by the structure, it is conceivable that water molecules find their way out of the structure. Moreover, the multi-temperature study also shows that the guest molecules inside $\alpha$-CD cavities are freely rotating with no apparent ordering induced by lowering the temperature. This is due to the lack of H-bond interactions between the guest and the host molecules, which, in theory, should help stabilising the position of the guest molecules. A CSD search reveals that the lack of direct intermolecular contacts between host and guest molecules is not unusual for CD inclusion complexes, and that the guest is indirectly H-bonded to the host via water molecules. 


\subsubsection{High-pressure results:}

Our high-pressure re-crystallisation attempts of $\alpha$-CD.SA inclusion complex were unsuccessful for the selected pressure and temperature ranges. It has been reported previously that " $\alpha$-CD (hydrate) shows an unusual trend in solubility as a function of pressure, that is, when increasing pressure crystals tend to dissolve rather than grow".${ }^{1} \mathrm{~A}$ similar behaviour was observed for $\alpha$-CD.SA inclusion complex with a partially dissolved crystal; however, unlike $\alpha$-CD hydrate, the crystal does not grow back with pressure release. The reason of the observed solubility trend is still unknown but currently under investigation and is believed to be associated with water mobility into $\alpha$-CD cavities through flip-flop H-bonding. ${ }^{1}$ On pressure release, there was also no microscopic evidence for crystallisation of either SA or $\alpha-\mathrm{CD}$ alone. Given the smaller size of the crystal at the end of the experiment, the solution in the DAC must become more saturated with respect to both components but a too high level of supersaturation could hinder crystallisation. It is also possible that kinetic effects are affecting crystallisation or that a particularly stable complex in solution is stabilised at high pressure. Finally, it is also conceivable that structural disorder is essential for stability of the $\alpha$-CD.SA complex and that high pressure, favouring denser, more ordered states, hinders the in situ formation of the complex.

\subsection{Conclusions}

The crystal structure of $\alpha$-CD.SA inclusion complex has been elucidated using singlecrystal X-ray diffraction. $\alpha$-CD molecules adopt a rounded shape allowing the complex to crystallise in a high symmetry space group, unreported previously for $\alpha$-CD inclusion complexes. The structure exhibits a honeycomb arrangement of channels. The building block of the channels has been described as a vase-like cluster formed by a trimer of $\alpha$-CD molecules.

The disorder of the sample has been assessed by means of a multi-temperature X-ray diffraction experiment. Careful analysis of Fourier maps proved that the disorder is of a dynamic nature. Structural comparison of $\alpha$-CD.SA complex at the different temperature shows that in the low-temperature regime disorder is attributed to thermal vibrations, however above a certain temperature vibrations of water molecules accentuate the disorder and lead eventually to the decomposition of the crystal. 


\subsection{References}

(1) Granero-García, R.; Lahoz, F. J.; Paulmann, C.; Saouane, S.; Fabbiani, F. P. a. A Novel Hydrate of $\alpha$-Cyclodextrin Crystallised under High-Pressure Conditions. CrystEngComm 2012, 8664-8670.

(2) Hejny, C.; Minkov, V. S. High-Pressure Crystallography of Periodic and Aperiodic Crystals. IUCrJ 2015, 2 (2), 218-229.

(3) Leeson, P. D.; Springthorpe, B. The Influence of Drug-like Concepts on Decision-Making in Medicinal Chemistry. Nat. Rev. Drug Discov. 2007, 6 (11), 881-890.

(4) Lipinski, C. a. Lead- and Drug-like Compounds: The Rule-of-Five Revolution. Drug Discov. Today Technol. 2004, 1 (4), 337-341.

(5) Zeikus, J. G.; Jain, M. K.; Elankovan, P. Biotechnology of Succinic Acid Production and Markets for Derived Industrial Products. Appl. Microbiol. Biotechnol. 1999, 51 (5), 545-552.

(6) Lisnyak, Y. V.; Martynov, A. V.; Baumer, V. N.; Shishkin, O. V.; Gubskaya, A. V. Crystal and Molecular Structure of $\beta$-Cyclodextrin Inclusion Complex with Succinic Acid. J. Incl. Phenom. Macrocycl. Chem. 2007, 58, 367-375.

(7) Martin Del Valle, E. M. Cyclodextrins and Their Uses: A Review. Process Biochem. 2003, 39 (9), 1033-1046.

(8) Harata, K.; Uekama, K.; Otagiri, M.; Hirayama, F. Crystal Structures Pentahydrate of aCyclodextrin-3-Lodopropionic Acid (1:1) Complex and Hexakis(2, 3, 6-Tri-O-Methyl)-aCyclodextrin- Lodoacetic Acid (1:1) Complex Monohydrate. Japan Chem. Soc. J. 1983, 2, 173.

(9) Tokuoka, R.; Abe, M.; Matsumoto, K.; Shirakawa, K.; Fujiwara, T.; Tomita, K. Structure of the $\alpha$-Cyclodextrin $(\alpha-\mathrm{CD})$ Inclusion Complex with the Potassium Salt of $\gamma$-Aminobutyric Acid (GABA). Acta Crystallogr. Sect. B Struct. Crystallogr. Cryst. Chem. 1981, 37 (2), 445-447.

(10) Sicard-Roselli, C.; Perly, B.; Le Bas, G. The Respective Benefits of X-Ray Crystallography and NMR for the Structural Determination of the Inclusion Complex Between ButylIsothiocyanate and. J. Incl. Phenom. Macrocycl. Chem. 2001, 39 (3-4), 333-337.

(11) Saenger, W.; Steiner, T. Cyclodextrin Inclusion Complexes: Host-Guest Interactions and Hydrogen-Bonding Networks. Acta Crystallogr. Sect. A Found. Crystallogr. 1998, 54 (6), 798805.

(12) Allen, F. H. The Cambridge Structural Database: A Quarter of a Million Crystal Structures and Rising. Acta Crystallogr. Sect. B Struct. Sci. 2002, 58, 380-388.

(13) Harata, K. Structural Aspects of Stereodifferentiation in the Solid State. Chem. Rev. 1998, 98 (5), 1803-1827.

(14) Alvarez, S. A Cartography of the van Der Waals Territories. Dalton Trans. 2013, 42 (24), 8617-8636. 
(15) Merrill, L.; Bassett, W. A. Miniature Diamond Anvil Pressure Cell for Single Crystal X-Ray Diffraction Studies. Rev. Sci. Instrum. 1974, 45, 290-294.

(16) Piermarini, G. J.; Block, S.; Barnett, J. D.; Forman, R. A. Calibration of the Pressure Dependence of the R1 Ruby Fluorescence Line to 195 Kbar. J. Appl. Phys. 1975, 46 (6), 27742780.

(17) Kottke, T.; Stalke, D. Crystal Handling at Low Temperatures. J. Appl. Crystallogr. 1993, 26 (pt 4), 615-619.

(18) Bruker-AXS. SAINT Version 8.34A, Madison, Wisconsin, USA. SAINT version 8.34A: Madison, Wisconsin, USA 2013.

(19) Sheldrick, G. M. SADABS Version 2008-1, Bruker-AXS, Madison, Wisconsin, USA. SADABS Version 2008-1, 2008, Bruker-AXS: Madison, Wisconsin, USA 2008.

(20) Sheldrick, G. M. A Short History of SHELX. Acta Crystallogr. Sect. A Found. Crystallogr. 2008, $64,112-122$.

(21) Sheldrick, G. M. Crystal Structure Refinement with SHELXL. Acta Crystallogr. Sect. C Struct. Chem. 2015, $71(\mathrm{Md}), 3-8$.

(22) Hübschle, C. B.; Sheldrick, G. M.; Dittrich, B. ShelXle: A Qt Graphical User Interface for SHELXL. J. Appl. Crystallogr. 2011, 44, 1281-1284.

(23) Grade Web Server, Global Phasing Ltd. http://grade.globalphasing.org/ (GRADE version 1.2.7).

(24) Thorn, A.; Dittrich, B.; Sheldrick, G. M. Enhanced Rigid-Bond Restraints. Acta Crystallogr. Sect. A Found. Crystallogr. 2012, 68, 448-451.

(25) Saenger, W.; Jacob, J.; Gessler, K.; Steiner, T.; Hoffmann, D.; Sanbe, H.; Koizumi, K.; Smith, S. M.; Takaha, T. Structures of the Common Cyclodextrins and Their Larger AnaloguesBeyond the Doughnut. Chem. Rev. 1998, 98 (5), 1787-1802.

(26) Ding, J.; Steiner, T.; Saenger, W. Structure of the $\gamma$-cyclodextrin-1-propanol-17H2O Inclusion Complex. Acta Crystallogr. Sect. B Struct. Sci. 1991, 47 (5), 731-738.

(27) Kamitori, S.; Hirotsu, K.; Higuchi, T. Crystal and Molecular Structures of Double Macrocyclic Inclusion Complexes Composed of Cyclodextrins, Crown Ethers, and Cations. J. Am. Chem. Soc. 1987, 109 (8), 2409-2414.

(28) Steiner, T.; Saenger, W. Channel-Type Crystal Packing in the Very Rare Space Group P4212 with $Z^{\prime}=3 / 4$ : Crystal Structure of the Complex $\gamma$-Cyclodextrin-Methanol-n-Hydrate. Acta Crystallogr. Sect. B Struct. Sci. 1998, 54 (4), 450-455.

(29) Chatziefthimiou, S. D.; Yannakopoulou, K.; Mavridis, I. M. $\beta$-Cyclodextrin Trimers Enclosing an Unusual Organization of Guest: The Inclusion Complex $\beta$-cyclodextrin/4Pyridinealdazine. CrystEngComm 2007, 9 (11), 976-979. 
(30) Cheng, X.; Lu, Z.; Li, Y.; Wang, Q.; Lu, C.; Meng, Q. An Interesting Molecular-Assembly of $\beta$-Cyclodextrin Pipelines with Embedded Hydrophilic Nickel Maleonitriledithiolate. Dalt. Trans. 2011, 40 (44), 11788-11794.

(31) Caira, M.; Bourne, S.; Mzondo, B. Cyclodextrin Inclusion Complexes of the Antioxidant $\alpha$ Lipoic Acid. Acta Crystallogr. Sect. A 2014, 70, C992.

(32) Liu, Y.; Zhong, R.-Q.; Zhang, H.-Y.; Song, H.-B. A Unique Tetramer of 4:5 $\beta$-Cyclodextrinferrocene in the Solid State. Chem. Commun. (Camb). 2005, No. 17, 2211-2213.

(33) Harada, A.; Li, J.; Kamachi, M.; Kitagawa, Y.; Katsube, Y. Structures of Polyrotaxane Models. Carbonhydrate Res. 1998, 305, 127-129.

(34) Saenger, W. Nature and Size of Included Guest Molecule Determines Architecture of Crystalline Cyclodextrin Host Matrixt. Isr. J. Chem. 1985, 25 (1), 43-50.

(35) Saenger, W. Cyclodextrin Inclusion Compounds in Research and Industry. Angew. Chemie Int. Ed. English 1980, 19 (5), 344-362.

(36) Harata, K. The Structure of the Cyclodextrin Complex. IX. The Crystal Structure of $\alpha$ Cyclodextrin-m-Nitroaniline (1:1) Hexahydrate Complex. Bull. Chem. Soc. Jpn. 1980, 53 (10), 2782-2786.

(37) Noltmeyer, N.; Saenger, W. Structural Chemistry of Linear a-Cyclodextrin-Polyiodide Complexes. (Li and Cd). J. Am. Chem. Soc. 1980, 102 (8), 2710-2722.

(38) Harata, K.; Uekama, K.; Otagir, M.; Hirayama, F.; Ogino, H. The Structure of the Cyclodextrin Complex. X. Crystal Structure of $\alpha$-Cyclodextrin-Benzaldehyde (1: 1) Complex Hexahyderate. Bull. Chem. Soc. Jpn. 1981, 54 (7), 1954-1959.

(39) Harata, K. The Structure of the Cyclodextrin Complex. XII. Crystal Structure of $\alpha$ Cyclodextrin-1-Phenylethanol (1:1) Tetrahydrate. Bull. Chem. Soc. Jpn. 1982, 55 (5), 13671371.

(40) Klingert, B.; Rihs, G. Molecular Encapsulation of Transition-Metal Complexes in Cyclodextrins. 1. Synthesis and X-Ray Crystal Structure of [(.eta.5-C5H5)Fe(.eta.6C6H6)]PF6.cntdot.2.alpha.-CD.cntdot.8H2O. Organometallics 1990, 9 (4), 1135-1141.

(41) Odagaki, Y.; Hirotsu, K.; Higuchi, T.; Harada, A.; Takahashi, S. X-Ray Structure of the $\alpha$ Cyclodextrin-ferrocene (2: 1) Inclusion Compound. J. Chem. Soc. Perkin 1 1990, No. 4, 1230 1231.

(42) Klingert, B.; Rihs, G. Molecular Encapsulation of Transition Metal Complexes in Cyclodextrins. Part 2. Synthesis and Crystal Structures of 2:1 Adducts between $\alpha$ Cyclodextrin and Metallocenium Hexafluorophosphates [ $75-\mathrm{C} 5 \mathrm{H} 5) 2 \mathrm{M}] \mathrm{PF} 6(\mathrm{M}=\mathrm{Fe}, \mathrm{Co}, \mathrm{Rh}$ ). J. Incl. Phenom. Mol. Recognit. Chem. 1991, 10 (2), 255-265.

(43) Chetcuti, P. A.; Moser, P.; Rihs, G. Metallacarborane Complexes as Guests for Cyclodextrins. Molecular Structure of the Inclusion Complex Cs[closo-3,3,3-(CO)3-3,1,2ReC2B9H11.cntdot..alpha.-CD].cntdot.8H2O. Organometallics 1991, 10 (8), 2895-2897. 
(44) Klingert, B.; Rihs, G. Molecular Encapsulation of Transition Metal Complexes in Cyclodextrins. Part 3. Structural Consequences of Varying the Guest Geometry in ChannelType Inclusion Compounds. J. Chem. Soc. Dalt. Trans. 1991, No. 10, 2749-2760.

(45) Shibakami, M.; Sekiya, A. X-Ray Crystallographic Study of Fluorine Atom Effect on Guest Orientation inside the $\alpha$-Cyclodextrin Cavity. Chem. Commun. 1992, 6 (1742), 1742-1743.

(46) Meister, G.; Stoeckli-Evans, H.; Süss-Fink, G. Einschlußverbindungen von Aromatruthenium-Komplexen Mit Cyclodextrinen. J. Organomet. Chem. 1993, 453 (2), 249253.

(47) Le Bas, G.; Mason, S. A. Neutron Diffraction Structure of $\alpha$-Cyclodextrin Cyclopentanone Hydrate at 20 K: Host-guest Interactive Disorder. Acta Crystallogr. Sect. B Struct. Sci. 1994, 50 (6), 717-724.

(48) Nicolis, I.; Villain, F.; Coleman, a. W.; De Rango, C. X-Ray Crystallographic Structure of the Two-to-One $\alpha$-Cyclodextrin-Acetone. 9H2O Compound. Supramol. Chem. 1994, 3 (4), 251259.

(49) Kamitori, S.; Muraoka, S.; Kondo, S.; Okuyama, K. Crystal Structures of Two Forms of a 2:1 Cyclomaltohexaose (Alpha-cyclodextrin)/4,4'-biphenyldicarboxylic Acid Inclusion Complex. Carbohydr. Res. 1998, 312 (4), 177-181.

(50) Muraoka, S.; Matsuzaka, O.; Kamitori, S.; Okuyama, K. Crystal Structures of Cyclomaltohexaose (??-Cyclodextrin) Complexes with P-Bromophenol and MBromophenol. Carbohydr. Res. 1999, 320 (3-4), 261-266.

(51) Harata, K.; Kawano, K. Crystal Structure of the Cyclomaltohexaose ( a -Cyclodextrin ) Complex with Isosorbide Dinitrate . Guest-Modulated Channel-Type Structure. Carbohydr. Res. 2002, 337, 537-547.

(52) Liu, Z.; Frasconi, M.; Lei, J.; Brown, Z. J.; Zhu, Z.; Cao, D.; Iehl, J.; Liu, G.; Fahrenbach, A. C.; Botros, Y. Y.; et al. Selective Isolation of Gold Facilitated by Second-Sphere Coordination with $\alpha$-Cyclodextrin. Nat. Commun. 2013, 4 (May), 1855.

(53) Steiner, T.; Saenger, W. No Title. Carbohydr. Lett. 1994, 1, 143.

(54) Choia, S.; Frankb, W.; Rittera, H. Novel Polymerization of Diethyl Fumarate and Maleate in Aqueous Media via Cyclodextrin-Complexes. React. Funct. Polym. 2006, 66 (1), 149-156.

(55) Gu, Z.-Y.; Guo, D.-S.; Liu, Y. Guest Releasing from Solution to Solid-State Triggered by Cyclomaltohexaose ( $\alpha$-Cyclodextrin) Aggregation. Carbohydr. Res. 2010, 345 (18), 2670-2675.

(56) Ceborskaa, M. Molecular Binding of $\alpha$-Cyclodextrin with 1,4-Dioxane in the Solid State. J. Carbohydr. Chem. 2014, 33 (1), 48-53.

(57) Chacko, K. K.; Saenger, W. Topography of Cyclodextrin Inclusion Complexes . 15 .' Crystal and Molecular Structure of the Cyclohexaamylose-7.57 Water Complex, Form 111. Fourand Six-Membered Circular Hydrogen Bonds. J Am Chem Soc 1981, 103 (3), 1708-1715. 
(58) Broadley, J. S.; Cruickshank, D. W. J.; Morrison, J. D.; Robertson, J. M.; Shearer, F. R. S.; Shearer, H. M. M. Three-Dimensional Refinement of the Structure of Beta-Succinic Acid. Proc. R. Soc. London. Ser. A 1959, 251 (1267), 441.

(59) Leviel, J. L.; Auvert, G.; Savariault, J. M. Hydrogen Bond Studies. A Neutron Diffraction Study of the Structures of Succinic Acid at 300 and 77 K. Acta Crystallogr. Sect. B Struct. Crystallogr. Cryst. Chem. 1981, 37 (3), 2185-2189.

(60) Srinivasa Gopalan, R.; Kumaradhas, P.; Kulkarni, G. U.; Rao, C. N. R. An Experimental Charge Density Study of Aliphatic Dicarboxylic Acids. J. Mol. Struct. 2000, 521, 97-106.

(61) Thalladi, V. R.; Nüsse, M.; Boese, R. The Melting Point Alternation in A, $\omega$ Alkanedicarboxylic Acids. J. Am. Chem. Soc. 2000, 122 (4), 9227-9236.

(62) Bhattacharya, S.; Saraswatula, V. G.; Saha, B. K. Thermal Expansion in Alkane Diacids Another Property Showing Alternation in an Odd-Even Series. Cryst. Growth Des. 2013, 13, 3651-3656.

(63) Macrae, C. F.; Bruno, I. J.; Chisholm, J. a.; Edgington, P. R.; McCabe, P.; Pidcock, E.; Rodriguez-Monge, L.; Taylor, R.; Van De Streek, J.; Wood, P. a. Mercury CSD 2.0 - New Features for the Visualization and Investigation of Crystal Structures. J. Appl. Crystallogr. 2008, 41 (2), 466-470.

(64) Gruene, T.; Hahn, H. W.; Luebben, A. V.; Meilleur, F.; Sheldrick, G. M. Refinement of Macromolecular Structures against Neutron Data with SHELXL2013. J. Appl. Crystallogr. 2014, 47, 462-466.

(65) Spek, A. L. PLATON SQUEEZE: A Tool for the Calculation of the Disordered Solvent Contribution to the Calculated Structure Factors. Acta Crystallogr. Sect. C Struct. Chem. 2015, 71, 9-18.

(66) Spek, A. L. Single-Crystal Structure Validation with the Program PLATON. J. Appl. Crystallogr. 2003, 36, 7-13.

(67) Spek, A. L. Structure Validation in Chemical Crystallography. Acta Crystallogr. Sect. D Biol. Crystallogr. 2009, 65, 148-155. 


\subsection{Appendix}

\subsubsection{CSD refcodes of the all $\alpha$-CD structures available in the CSD:}

The following is a list of all the recodes resulting of searching the CSD, V. 5.36 including updates to November 2014, for structure of $\alpha$-CD with 3-D coordinates. The refcodes were categorised according to the packing type described by Saenger and Steiner. ${ }^{11}$

a) Herringbone-type cage: ARACEB, BANXUJ, BOBPAK, BUPDEV, CDEXKR10, CDEXME10, CDEXTI10, CHXAMH, CHXAMH02 (redetermination, excluded), CHXAMH03, CHXAMH04 (redetermination, excluded), CYDXKR10, DAXTEB, DEGTEO, GEVTOQ, GULTUC, HOGCIP, LOVVAT, QERVUE, QOHMEF, RAXPOV, REGPAW, YENQAK.

b) Brick-type cage: ACDHBA, ACDMFM, ACDMSM, ACDPNP, ACDPRO, BOBNUC, BOLVUT, BORYOX, CDEXIA01 (redetermination, excluded), CDEXIA10, CHAIPL, EJOVOO, GOQZUH, IFOBUB, INUPAI, INUPEM, MESYEO, PAZKOS, QENTIN, QENTOT, QIZMIW, RIRZUN ( $\alpha$-cycloaltrin, excluded), TUSHEV, TUSHIZ, VEHQAA, WEXKOZ, ZEJDEX.

c) Channel type (pseudohexagonal and hexagonal/honeycomb): BAJJAX, BIJHOR, CAQPEP, CDNOAN, CIGLAG, CYDXLI10, JEHYIE, JEMGUD, JUMYOF, JUMYUL, KIRJOK10, KIWYUK, KIWZEV, KOBLOC, KOGKEW, KOGKIA, LIZQAO, MOVFUY, PEPBUH, PUPTEZ, QACCEE, QACCII, WEXLEQ, XIGBOE, YIHJOQ, YIHJUW, ZASYOH. Channel type (other): ACDMNP, ACDPRS, CAQPAL, CDKABA, CDXBZS, CDXKOM, CDXSOM, CHAMPA, EJOVUU, KEZGAZ, MESYAK, NIMROR, QOHMIJ, RAGZOO, SAQMII, TEXTEX, TEXTIB, WILJAC, WIZQEB, WUQKEZ, WUQKID, WUQKOJ, ZZZANG10.

\subsubsection{The evolution of the normalised unit cell parameters as function of temperature}

The normalised unit cell parameters are plotted against the temperature. Data at $100 \mathrm{~K}$ were taken as a reference $\left(a_{100}=a_{0}, c_{100}=c_{0}\right.$ and $\left.V_{100}=V_{0}\right)$. To avoid the overlap of the error bars, $T_{c}$ and Tv were shifted by +1 and $-1 \mathrm{~K}$, respectively. The uncertainty of the ratio $\sigma\left(a / a_{0}\right)$ was calculated using the equation of the propagation of uncertainty where:

$$
\sigma\left(\frac{a}{a_{0}}\right)=\sqrt[2]{\left(\frac{\sigma a}{a}\right)^{2}+\left(\frac{\sigma a_{0}}{a_{0}}\right)^{2}}
$$

The same equation was used for generating $\sigma\left(c / c_{0}\right)$ and $\sigma\left(V / V_{0}\right)$. 
Table 3.2: evolution of the normalised unit cell parameters as function of temperature.

\begin{tabular}{ccccc}
$\mathbf{T} / \mathbf{K}$ & $\mathrm{a} / \AA$ & $\sigma(\mathrm{a}) / \AA$ & $\mathrm{a} / \mathrm{a} 0$ & $3 \cdot \sigma(\mathrm{a} / \mathrm{a} 0)$ \\
\hline $\mathbf{1 0 0}$ & 23.645 & 0.003 & 1 & 0.000538 \\
$\mathbf{1 2 0}$ & 23.6608 & 0.0007 & 1.000668 & 0.000391 \\
\hline $\mathbf{1 5 0}$ & 23.6781 & 0.0006 & 1.0014 & 0.000388 \\
$\mathbf{1 8 0}$ & 23.6951 & 0.0005 & 1.002119 & 0.000386 \\
\hline $\mathbf{2 1 0}$ & 23.7133 & 0.0006 & 1.002889 & 0.000388 \\
\hline $\mathbf{2 4 0}$ & 23.738 & 0.013 & 1.003933 & 0.001686 \\
\hline $\mathbf{2 7 0}$ & 23.757 & 0.01 & 1.004737 & 0.001319 \\
\hline $\mathbf{T} / \mathrm{K}$ & $\mathrm{c} / \AA$ & $\sigma(\mathrm{c}) / \AA$ & $\mathrm{c} / \mathrm{c} 0$ & $3 \cdot \sigma(\mathrm{c} / \mathrm{c} 0)$ \\
\hline $\mathbf{1 0 1}$ & 55.706 & 0.00700 & 1 & 0.000533 \\
\hline $\mathbf{1 2 1}$ & 55.722 & 0.00200 & 1.000287 & 0.000392 \\
\hline $\mathbf{1 5 1}$ & 55.7898 & 0.00180 & 1.001504 & 0.000389 \\
\hline $\mathbf{1 8 1}$ & 55.849 & 0.00150 & 1.002567 & 0.000385 \\
\hline $\mathbf{2 1 1}$ & 55.8345 & 0.00170 & 1.002307 & 0.000388 \\
\hline $\mathbf{2 4 1}$ & 55.76 & 0.03000 & 1.000969 & 0.001657 \\
\hline $\mathbf{2 7 1}$ & 55.62 & 0.02000 & 0.998456 & 0.001143 \\
\hline $\mathbf{T} / \mathrm{K}$ & $\mathrm{V} / \AA$ & $\sigma \mathrm{V} / \AA$ & $\mathrm{V} / \mathrm{V} 0$ & $3 \cdot \sigma(\mathrm{V} / \mathrm{V} 0)$ \\
\hline $\mathbf{9 9}$ & 26973 & 7 & 1 & 0.001101 \\
\hline $\mathbf{1 1 9}$ & 27015.8 & 1.9 & 1.001587 & 0.000807 \\
\hline $\mathbf{1 4 9}$ & 27088.1 & 1.6 & 1.004267 & 0.000798 \\
\hline $\mathbf{1 7 9}$ & 27155.8 & 1.4 & 1.006777 & 0.000794 \\
\hline $\mathbf{2 0 9}$ & 27190.5 & 1.6 & 1.008064 & 0.000798 \\
\hline $\mathbf{2 3 9}$ & 27212 & 34 & 1.008861 & 0.003828 \\
\hline $\mathbf{2 6 9}$ & 27183 & 26 & 1.007786 & 0.002973 \\
\hline & & & & \\
\hline
\end{tabular}




\subsubsection{Analysis of the anisotropic displacement parameters}

The value of the ADPs were taken from the result file of the refinement of SHELX (.res). $\mathrm{O}(1)$ to $\mathrm{O}(6)$ are water molecules located in the interstices. The numbering suffix refers to the residue number in the structure.

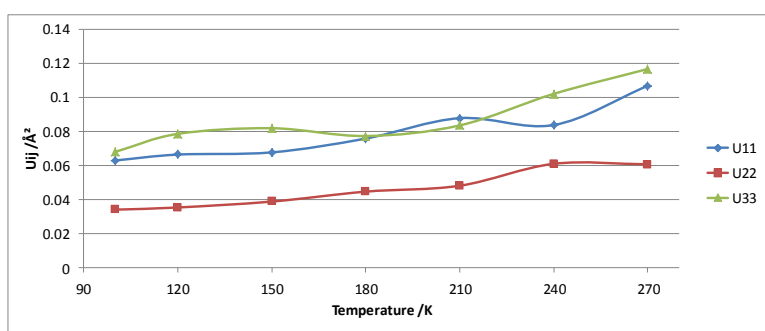

Figure 3.12. $\mathrm{U}_{\mathrm{ij}}$ of the water molecule $\mathbf{O ( 1 )}$ _90

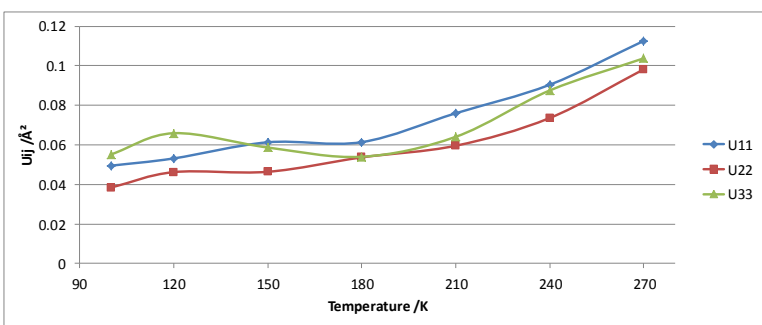

Figure 3.13. $\mathrm{U}_{\mathrm{ij}}$ of the water molecule $\mathrm{O}(2) \_90$

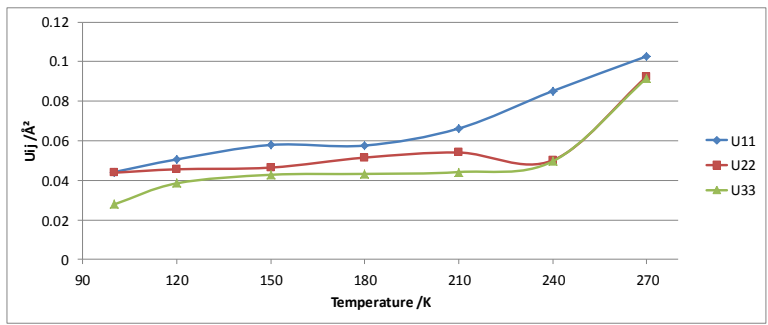

Figure 3.14. $\mathrm{U}_{\mathrm{ij}}$ of the water molecule $\mathrm{O}(3) \_91$

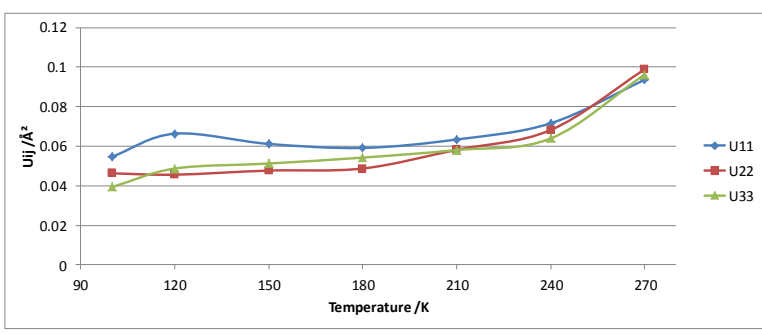

Figure 3.15. $\mathrm{U}_{\mathrm{ij}}$ of the water molecule $\mathrm{O}(4) \_91$

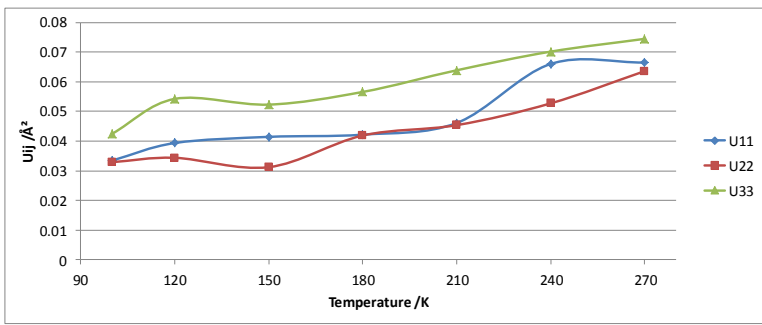

Figure 3.16. $\mathrm{U}_{\mathrm{ij}}$ of the water molecule $\mathrm{O}(5) \_91$

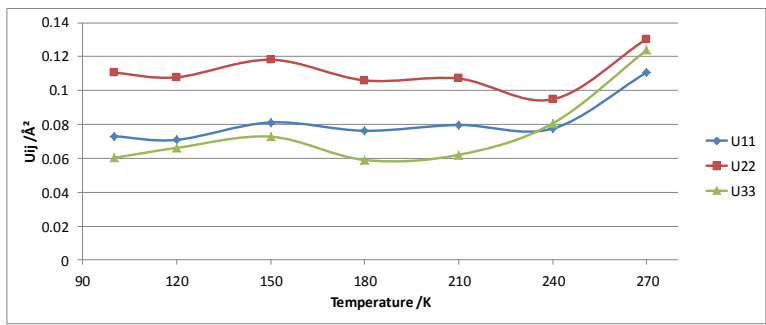

Figure 3.17. $\mathrm{U}_{\mathrm{ij}}$ of the water molecule $\mathrm{O(6) \_ 91}$

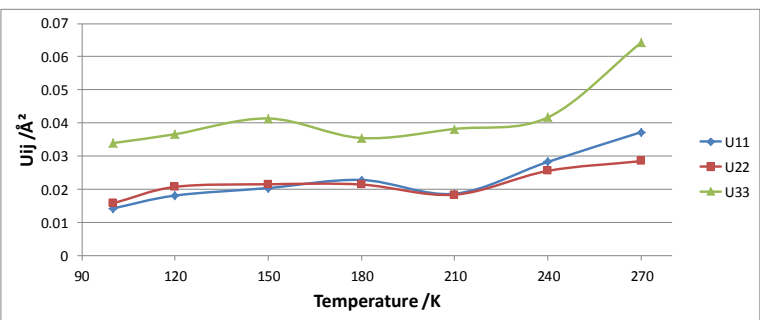

Figure 3.18. $\mathrm{U}_{\mathrm{ij}}$ of the C-atom $\mathbf{C}(\mathbf{1}) \_11$ of $\mathbf{A} \alpha-\mathrm{CD}$ 


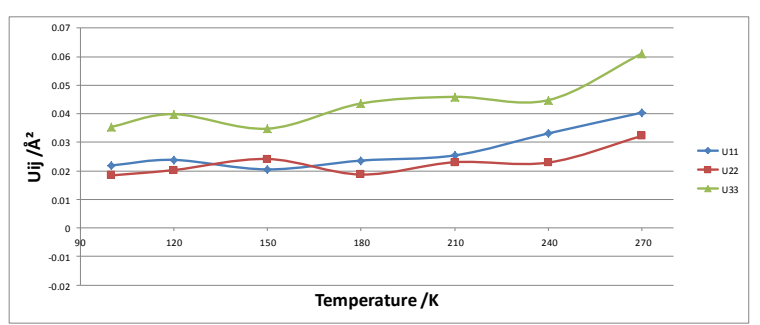

Figure 3.19. $\mathrm{U}_{\mathrm{ij}}$ of the C-atom C(1)_21 of $\mathbf{B} \alpha$-CD

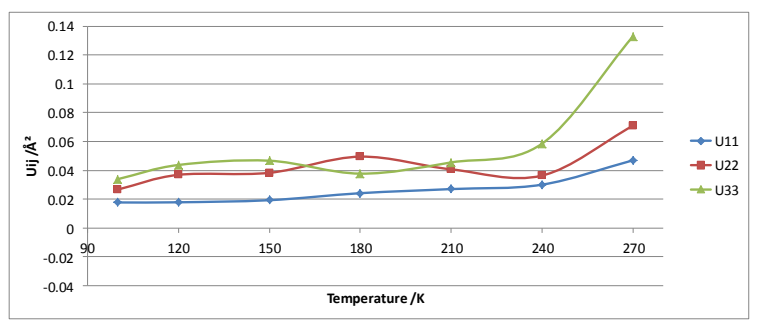

Figure 3.20. $\mathrm{U}_{\mathrm{ij}}$ of the $\mathrm{C}$-atom $\mathbf{C}(\mathbf{1}) \_31$ of $\mathbf{C} \alpha$-CD

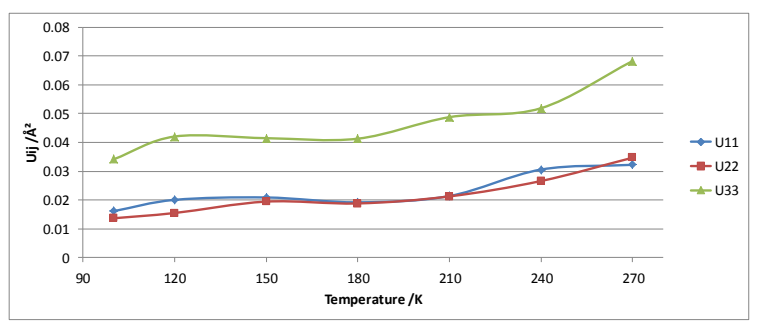

Figure 3.21. $\mathrm{U}_{\mathrm{ij}}$ of the O-atom $\mathbf{O}(4) \_11$ of $\mathbf{A} \alpha-\mathrm{CD}$

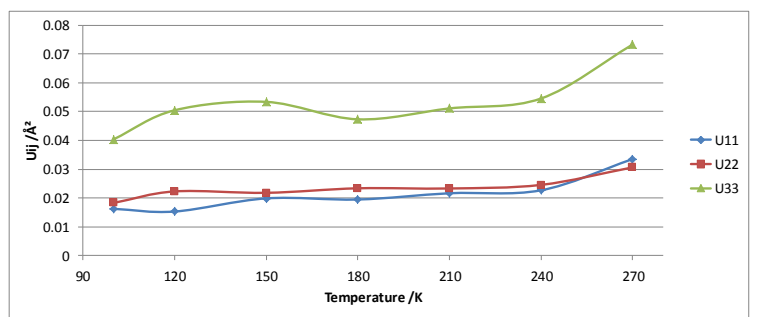

Figure 3.22. $\mathrm{U}_{\mathrm{ij}}$ of the $\mathrm{O}$-atom $\mathbf{O}(4) \_12$ of $\mathbf{A} \alpha$-CD

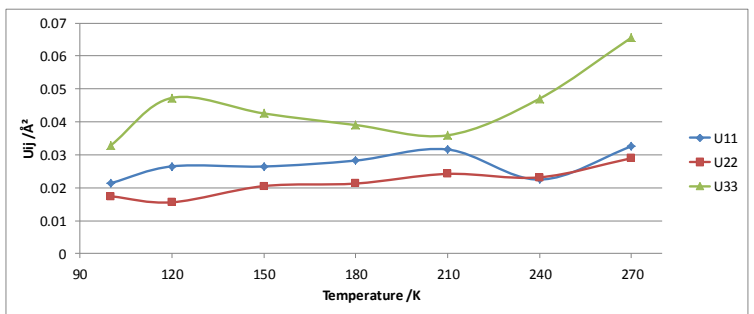

Figure 3.23. $\mathrm{U}_{\mathrm{ij}}$ of the O-atom $\mathbf{O}(4) \_21$ of $\mathbf{B} \alpha$-CD

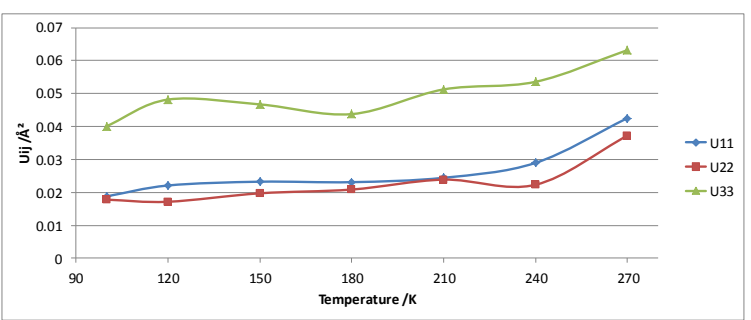

Figure 3.24. $\mathrm{U}_{\mathrm{ij}}$ of the O-atom $\mathbf{O}(4) \_22$ of $\mathbf{B} \alpha-\mathrm{CD}$

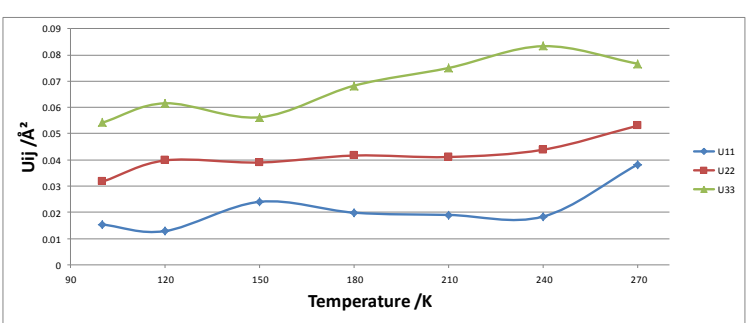

Figure 3.25. $\mathrm{U}_{\mathrm{ij}}$ of the $\mathrm{O}$-atom $\mathbf{O}(4) \_31$ of $\mathrm{C} \alpha$-CD

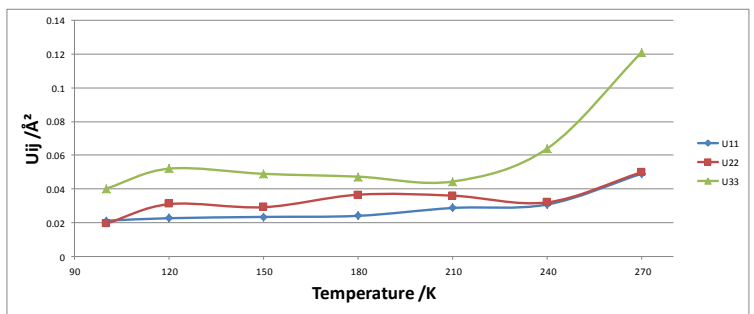

Figure 3.26. $\mathrm{U}_{\mathrm{ij}}$ of the $\mathrm{O}$-atom $\mathbf{O}(4) \_32$ of $\mathbf{C} \alpha-\mathrm{CD}$ 


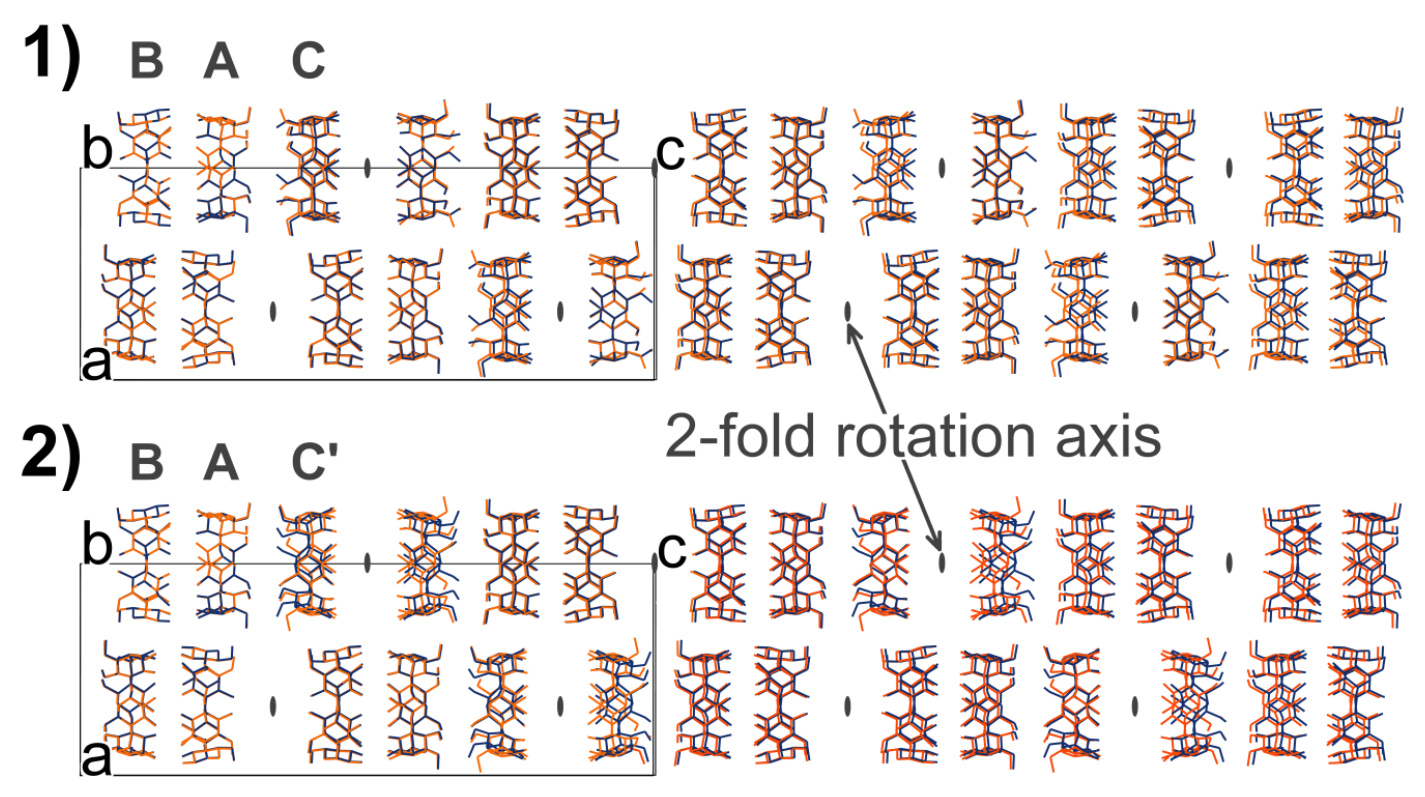

Figure 3.27. Overlay of $\alpha$-CD.SA structures at $180 \mathrm{~K}$ and $270 \mathrm{~K}$ viewed along the $b$-axis. (1) overlay using $\alpha$ CD C with a site occupancy of $0.689(10)$; (2) overlay using $\alpha$-CD $C^{\prime}$ with a site occupancy of $0.311(10)$ 


\section{Chapter 4. A new high-pressure form of $\beta$-cyclodextrin'paracetamol inclusion complex}

\subsection{Synopsis}

In this chapter, a new form of a $\beta$-CD·paracetamol inclusion complex obtained exclusively at high pressure is reported. This form, here termed form $\mathrm{V}$, was obtained by first dissolving in situ in a DAC the ambient-pressure form of the complex, form I, and then further increasing pressure to $0.8 \mathrm{GPa}$ to induce crystallisation. High-pressure single-crystal diffraction data were collected using both laboratory and synchrotron $\mathrm{X}$-ray radiation. The presence of the guest in the cyclodextrin cavity was also confirmed by Raman spectroscopy. One of the most striking structural features of form $\mathrm{V}$ is the exceptionally high number of water molecules, over 24 per inclusion complex unit, incorporated in the crystal. Comparative crystal structure analysis indicates that the mode of packing in form $\mathrm{V}$ is intermediate between the known screw channel and chessboard packing types. The results of compression studies of form I using different pressure-transmitting media are also reported in this chapter; these studies revealed that no phase transition occurs up to $0.95 \mathrm{GPa}$ but that structure of form I responds differently according to the nature of the pressuretransmitting medium used.

\subsection{Introduction}

Among the natural occurring $\alpha$-, $\beta$ - and $\gamma$-cyclodextrins (CD), $\beta$-CD (Figure 4.1.b) is the most frequently investigated with respect to inclusion complex formation. $\beta$-CD is composed of seven D-glucopyranose units (Figure 4.1.c) linked together by $\alpha(1 \rightarrow 4)$ glycosidic bonds (Figure 4.1.b). Within the family of native $\mathrm{CDs}, \beta-\mathrm{CD}$ is the least soluble in water with a reported solubility value of $18.5 \mathrm{~g} / \mathrm{l}$, compared to $\alpha$ - and $\gamma$-CDs with values of 145 and $232 \mathrm{~g} / \mathrm{l}$, respectively. ${ }^{1}$ It seems paradoxical to report that on one hand $\beta-\mathrm{CD}$ is the most used $\mathrm{CD}$ to improve drug solubility in water, ${ }^{2-9}$ and on the other hand it is a poorly soluble natural CD. Chen et al. reported, by calculating the host-guest binding energy and comparing it to experimental data, ${ }^{10}$ that $\beta-\mathrm{CD}$, when compared to $\alpha$ - and $\gamma$-CD, binds in a more efficient manner to guest molecules. ${ }^{11}$ Moreover, $\alpha$-CD and $\gamma-\mathrm{CD}$ cavities are respectively too small and too large to incorporate and stabilise as many drug compounds as $\beta$-CD. Another important reason why $\beta$-CD is favoured over $\alpha$ - and $\gamma$ $\mathrm{CDs}$ is the production cost: the low water solubility of $\beta$-CD makes the purification process much simpler, and hence more cost effective. ${ }^{12,13}$

Although cyclodextrins have been extensively studied as potential drug carriers, as described in several reviews, 2,4-8,14 the use of high-pressure techniques to extend the investigations 
on host-guest inclusion complex formation was, to the best of our knowledge, prior to our work largely unexplored. Inclusion complex formation and phase transitions investigations have been reported at ambient and low-temperature conditions only. Here we demonstrate that highpressure techniques can be used to add a further dimension in the experimental search of inclusion complexes.
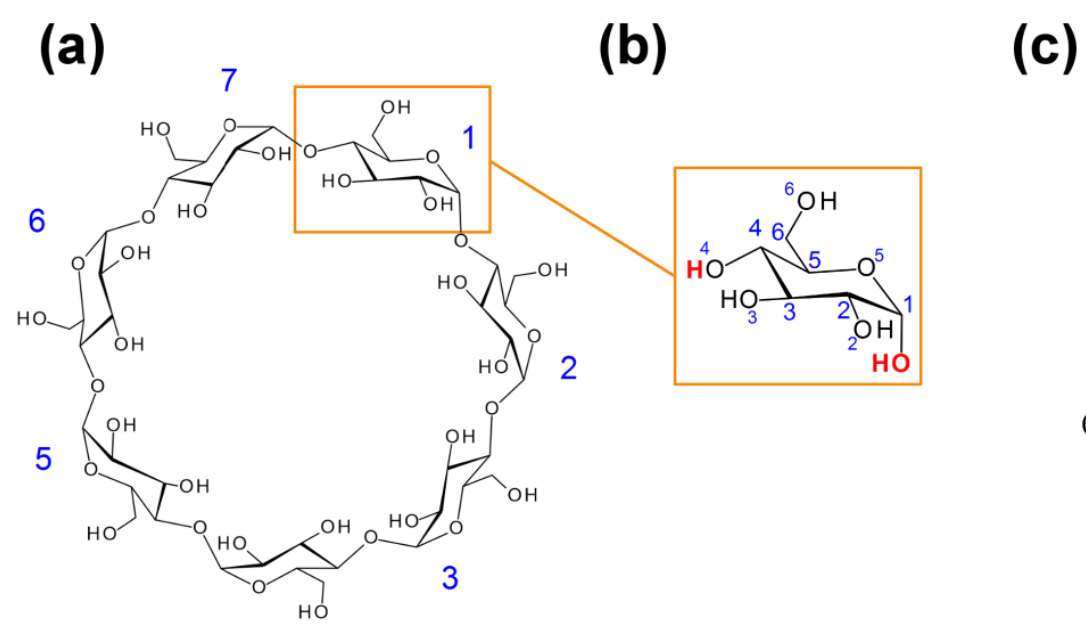<smiles>CC(=O)Nc1ccc(O)cc1</smiles>

Figure 4.1. Diagrams and numbering schemes of: (a) $\beta$-CD; (b) $\alpha$-D-glucopyranose and (c) paracetamol.

The work presented in this chapter focuses exclusively on the $\beta$-CD.paracetamol system. Although other drug molecules have been investigated, see Chapter 5, a large portion of time was dedicated to paracetamol (Figure 4.1.a). The motivation for this work is multifold: first of all, paracetamol has been known for decades as an effective analgesic and antipyretic substance, and is such an emblematic drug molecule that is often used as a model molecule for teaching purposes. ${ }^{15}$ Second, paracetamol is known to form a complex with $\beta$-CD at conditions of ambient pressure and temperature, here termed form I.16 Finally, the work carried out in our group has shown that highpressure and low-temperature crystallisation techniques lead to the formation of three further forms: form II and III obtained at low temperature and form IV obtained at high pressure, ${ }^{17}$ as summarised in Figure 4.2.

The aim of this study can be summarised as follows:

a) Confirm and tune the high-pressure crystallisation protocol of form IV; ${ }^{17}$

b) Check whether direct compression can induce the form I $\rightarrow$ II transition. The literature shows that polymorphs of molecular crystals obtained at low-temperature conditions can sometimes be obtained at high pressure as well. ${ }^{18}$

c) Investigate whether new forms can be obtained by varying parameters such as pressure, temperature and concentration. 


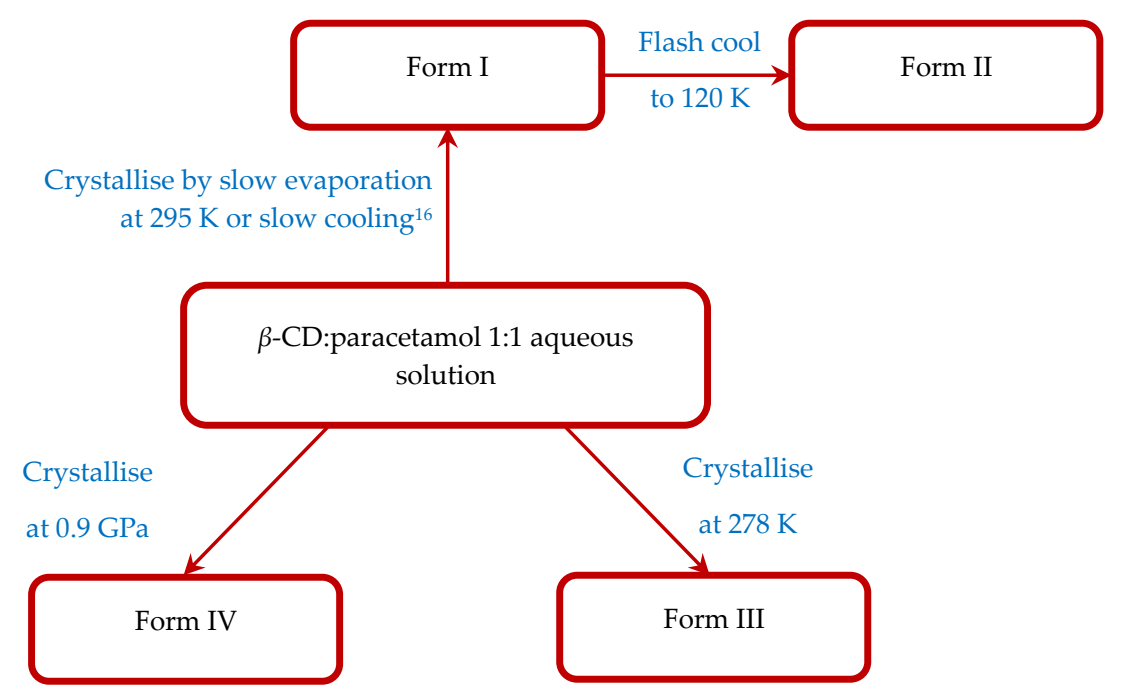

Figure 4.2. Crystallisations procedures of previously known $\beta$-CD·paracetamol inclusion complexes

\subsection{Experimental}

\subsubsection{Ambient-pressure crystallisation}

The crystallisation of the known $\beta$-CD.paracetamol complex, form $\mathrm{I}$, is very facile. It was described one and a half decades ago by Caira and Dodds and involves slow cooling of a $\beta$-CD and paracetamol equimolar aqueous solution, which yields large prismatic crystals. ${ }^{16}$ We found that slow evaporation instead of slow cooling also yields crystals suitable for diffraction. Single-crystal X-ray data were collected in house to redetermine the structure of form I at ambient-temperature and ambient-pressure conditions.

\subsubsection{High-pressure in situ crystallisation of $\beta$-CD·paracetamol form $\mathrm{V}$}

Crystallisation of the $\beta$-CD.paracetamol-water system at high pressure is complex. Numerous crystallisation trials were carried out in the DAC; three starting conditions were tested and used for the trials: (1) loading small single crystals of $\beta$-CD.paracetamol form I together with their mother liquor; (2) loading a large single crystal of form I, which fills more than $60 \sim 70 \%$ of the gasket hole, in demineralised water; (3) loading a hot supersaturated equimolar aqueous solution of $\beta$-CD.paracetamol (ca. $353 \mathrm{~K}$ ). The concentration of $\beta$-CD and paracetamol in the DAC is highest in trials (1) and lowest in trials (3).

In (1) and (2), the crystals of form I undergo an unexpected dissolution process at pressures higher than 0.2 GPa. Dissolution is generally complete above $0.5 \mathrm{GPa}$. In-situ crystallisation of a new $\beta$-CD.paracetamol-water phase, form $\mathrm{V}$, is observed at pressures above $0.6 \mathrm{GPa}$. When pressure is released, the reverse process is observed: form $\mathrm{V}$ dissolves at pressures below $0.6 \mathrm{GPa}$ and form I recrystallises back from solution. Another crystallisation path for obtaining form $\mathrm{V}$ consists of loading crystals as described in (1) (Figure 4.3.a), heating the sample in the DAC (Figure 
4.3.b-d) at pressures below 0.2 GPa using a heatgun until complete dissolution (Figure 4.3.e), and then increasing pressure above 0.6 GPa (Figure 4.3.f) to induce crystallisation.

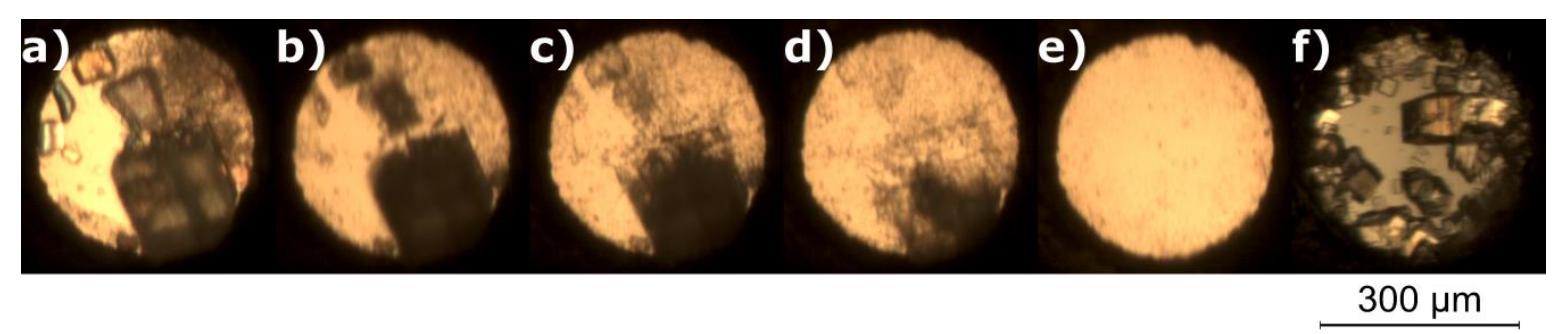

Figure 4.3. Dissolution process of $\beta$-CD.paracetamol form I. (a) Single crystals of $\beta$-CD.paracetamol form I loaded in their mother liquor into the DAC, P adjusted to $0.2 \mathrm{GPa}$; (b-e) progressive dissolution of the crystals in (a) by heating at $373 \mathrm{~K}$ at $0.2 \mathrm{GPa}$; (f) a new high pressure phase crystallises after increasing the pressure to $0.98 \mathrm{GPa}$.

The experiment in (3) was designed to reproduce the crystallisation of form IV and explore the formation of other forms. ${ }^{17}$ This procedure failed to reproduce the crystallisation of form IV, with only small crystals of form $\mathrm{V}$ obtained instead (Figure 4.4), as obtained by synchrotron singlecrystal X-ray diffraction. Crystallisation of needle-like crystals was observed when pressure was increased rapidly in one step to 1.2 GPa. These crystals correspond to a monohydrate form of paracetamol (see Chapter 7), which can be dissolved by releasing pressure to $0.6 \mathrm{GPa}$; on further slow pressurisation, crystallisation of form $\mathrm{V}$ can be induced.

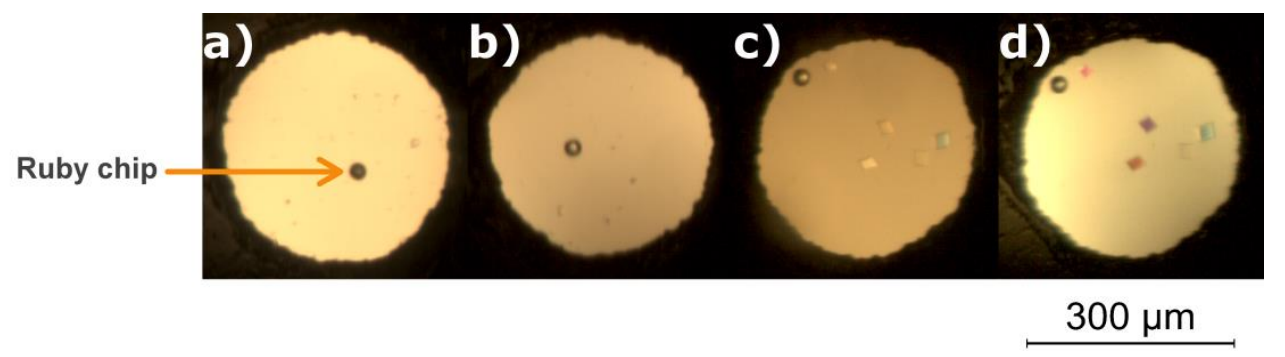

Figure 4.4. Crystallisation of $\beta$-CD.paracetamol form V from saturated solution. (a) hot saturated solution of $\beta$ $\mathrm{CD}$ :paracetamol 1:1 loaded into a DAC and pressure increased to $0.14 \mathrm{GPa}$; (b) no crystallisation after increasing pressure to $0.86 \mathrm{GPa}$; (c) crystallisation after increasing pressure to $0.94 \mathrm{GPa}$ and cooling to $285 \mathrm{~K}$ for $16 \mathrm{~h}$; $(\mathrm{d})$ maximum growth of crystals after 2 days at room temperature.

After several experiments, the chosen single crystal of form $\mathrm{V}$ for diffraction measurement (Figure 4.5. Crystal 1) was obtained by loading a large $(250 \times 180 \times 100 \mu \mathrm{m})$ single crystal of form I into the DAC and using demineralised water as pressure transmitting medium. The crystal filled more than $70 \%$ of the gasket hole. Pressure was gradually increased to dissolve the crystal and after 12 hours crystallisation of form $\mathrm{V}$ was complete at a pressure of $0.8 \mathrm{GPa}$. Subsequent experiments showed that cycling temperature on form $\mathrm{V}$ crystals to grow one single crystal always led to the

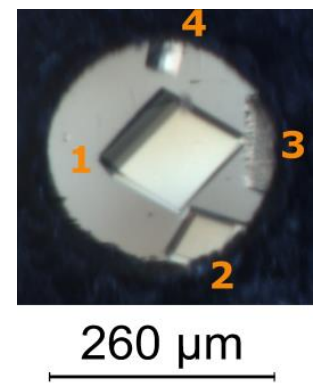

Figure 4.5. Crystals of $\beta$-CD·paracetamol form $\mathrm{V}$ crystallised at $0.8 \mathrm{GPa}$. 
formation of multiple nucleation sites; cycling temperature was thus not investigated further.

\subsubsection{X-ray diffraction and Raman spectroscopy}

\subsubsection{In-house X-ray diffraction}

Unit cell determination and diffraction quality check of $\beta$-CD.paracetamol form $\mathrm{V}$ were carried out in-house using a Bruker AXS SMART Apex II CCD diffractometer equipped with Mo K $\alpha$ sealed-tube radiation of $\lambda=0.71073 \AA$. To improve access to reciprocal space and better characterise the inclusion complex, synchrotron radiation was favoured over in house measurements. However, low-order data were collected on the same Bruker machine to complete and improve the saturated low angle reflections collected at the Doris and Petra synchrotron.

\subsubsection{Synchrotron X-ray diffraction}

Data on form V (Figure 4.5. Crystal 1) were collected using a wavelength of $0.50000 \AA$ at beamline F1 of the former DORIS synchrotron ring at Hasylab/DESY (Hamburg, Germany). A Huber 4-circle diffractometer equipped with a Mar165 CCD detector was used for the experiment. Using available slits, a beam of $150 \times 150 \mu \mathrm{m}$ was chosen to minimise diffraction from the gasket.

Crystal 1 (Figure 4.5) was measured using four runs ( $\varphi$ scans) by choosing an exposure time of $60 \mathrm{~s}$ per frame with a $\Delta \varphi$ of $0.5^{\circ}$. Two different orientations of the DAC were measured to improve completeness $(71.7 \%)$ and redundancy (13.94 up to $d_{\max }=1.1 \AA$ ). Data were converted from TIF to Bruker format using the programs $C M H$, which updated the frames' headers with information about the diffractometer angles and monitor counts, and $M 2 B$, which converts MarCCD165 data frames to Bruker-Nonius Smart format. ${ }^{19}$ Subsequently, the latter were converted to Bruker SFRM frame format using the conversion script implemented into the APEXII software. ${ }^{20}$

In an attempt to improve the resolution of data measured at F1, diffraction data were measured a second time at the extreme conditions beamline P02.2 of the PETRA synchrotron ring at DESY (Hamburg, Germany). The high brilliance of the microfocused beam $(\lambda=0.28962 \AA, 5 \times 2$ $\mu \mathrm{m})$ and the large amorphous silicon Perkin Elmer XRD 1621 detector enabled data collection up to

a $d_{\max }$ of $1.0 \AA$. Data were collected on a single orientation of the DAC using $1 \mathrm{~s}, 0.5^{\circ} \varphi$ scans covering a total of $82^{\circ}$. Two issues were observed by using the flat panel detector: 1) so-called "ghost peaks", which originate from a previous high dose of X-rays or very fast acquisition time; ${ }^{21}$ ghost peaks have intensities much lower than that of the mean intensity of the background because of a change in the detector pixel sensitivity;22 and 2) very strong diamond reflections, which generate a large vertical band of high intensity across the image. Hence, careful data integration and analysis of the $F_{\text {calc }}$ vs. Fobs plot during structure refinement was carried out; suspected ill reflections were inspected on the frames and omitted if needed. 
Following the Raman spectroscopy results (see section 4.4.4.2), X-ray data were collected for the small crystal 3 (Figure 4.5) using $1 \mathrm{~s}$ exposure, $2^{\circ} \varphi$ rotation scans covering a total of $80^{\circ}$.

\subsubsection{Compression studies of form I:}

In order to investigate a possible single-crystal to single-crystal phase transition in $\beta$ CD.paracetamol form I triggered by applying high pressure, two compression studies were carried out using perfluorotributylamine (FC43), and tetrahydrofuran (THF) as pressure transmitting medium. A single crystal of $\beta$-CD·paracetamol form I was loaded into a DAC filled with FC43, and X-ray diffraction was carried out at Doris F1 beamline $(\lambda=0.50000 \AA)$ : data were collected on the single crystal at $0.13 \mathrm{GPa}$ using two orientations of the DAC and $40 \mathrm{~s}$ exposure, $0.5^{\circ} \varphi$ rotation scans, and at $0.95 \mathrm{GPa}$ also using two orientations of the DAC, $80 \mathrm{~s}$ exposure, $1^{\circ} \varphi$ rotation scans. Changes in unit-cell parameters were monitored at intermediate pressures $(0.45,0.61$ and 0.78 GPa). For the compression study using THF as pressure transmitting medium, data were collected at PETRA 02.2 beamline $(\lambda=0.28962 \AA)$; data sets were collected at three pressure points $0.16,0.6$ and $0.95 \mathrm{GPa}$ (Figure 4.6) using $1 \mathrm{~s}$ exposure, $1^{\circ} \varphi$ rotation scans covering a total of $80^{\circ}$. Data were collected using two orientations of the DAC at $0.16 \mathrm{GPa}$, three orientations at $0.6 \mathrm{GPa}$ and a single one at $0.95 \mathrm{GPa}$.
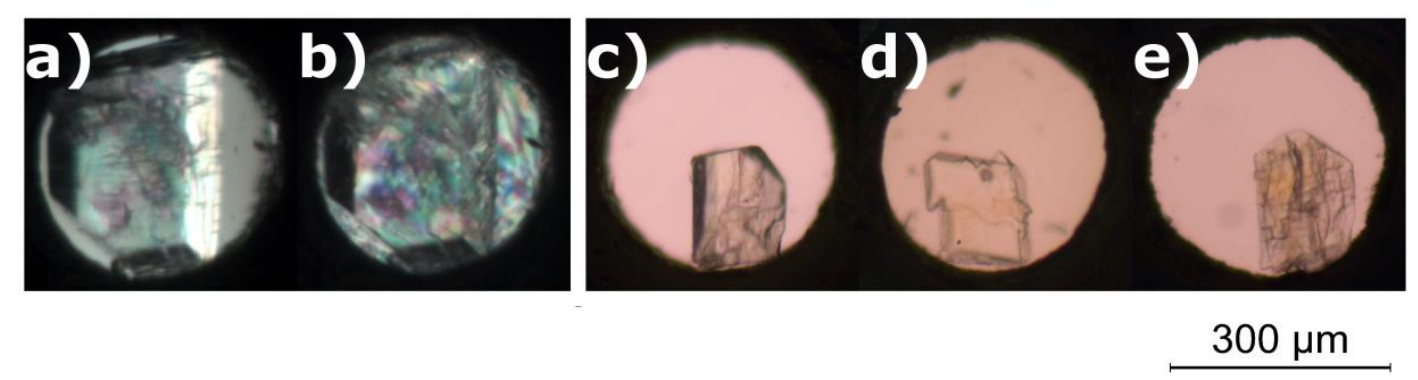

Figure 4.6. Compression study of $\beta$-CD-paracetamol form I using FC43 and THF. (a-b) FC43: (a) single crystal of form I at $0.13 \mathrm{GPa}$, (b) the same single crystal at $0.95 \mathrm{GPa}$; (c-e) THF: (c,e) a single crystal of form I at 0.16 and $1.02 \mathrm{GPa}$; (d) a second single crystal of form I at $0.6 \mathrm{GPa}$.

\subsubsection{Data processing}

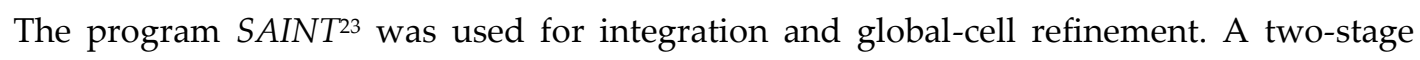
absorption correction was performed with $S H A D E^{24}$ and $S A D A B S^{25}$. Friedel pairs in all data sets (measured in house and the synchrotron) were merged with $S A D A B S^{25}$ assuming the effects of anomalous dispersion are negligible. All structures were solved with macromolecular phasing methods using SHELXD. ${ }^{26}$ Fragment seeding using the $\beta$-CD backbone (the 3D coordinates of the $\mathrm{CSD}^{27}$ refcode FALDUS ${ }^{28}$ were used as seeding model; H-atoms, all hydroxy groups and water molecules were deleted before use). Full-matrix least-squares structure refinement against $F^{2}$ was performed using SHELXL201426 through the SHELXLE GUI. ${ }^{29}$ Stereochemical restraints for the host 
and guest molecules were applied to increase the stability of the refinement: restraints were generated by the GRADE program using the GRADE Web server. ${ }^{30}$ A GRADE dictionary for SHELXL contains target values and standard deviations for 1,2-distances (DFIX) and 1,3-distances (DANG), as well as restraints for planar groups (FLAT). Anisotropic displacement parameters were refined for only the host molecules using the new rigid bond restraint (RIGU) implemented in the SHELXL2014 program. ${ }^{31}$ All H-atoms of the guest and host molecules were placed geometrically using the riding model. H-atoms belonging to water molecules were not visible in difference Fourier maps but were taken into account for calculating $F_{000}$ and density. $U_{\text {iso }}(\mathrm{H})$ values were assigned in the range 1.2-1.5 times $U_{\text {eq }}$ of the parent atom. Full details on the treatment of disorder can be found below.

\subsubsection{Raman spectroscopy}

Raman spectra were measured on a Horiba Jobin Yvon HR800 UV Micro-Raman spectrometer equipped with an air-cooled $20 \mathrm{~mW} 488 \mathrm{~nm}$ Ar-laser. Spectra were collected in the $100-3200 \mathrm{~cm}^{-1}$ range with a spectral resolution of $c a .2 .2 \mathrm{~cm}^{-1}$ using a grating of 600 grooves per mm and a Peltier-cooled CCD (Andor, 1024×256 pixels). The spectra were calibrated with the Raman scattering frequency of Si before and after each measurement.

\subsection{Results and discussion}

\subsubsection{Description of the structure}

Form $\mathrm{V}$ of $\beta$-CD.paracetamol inclusion complex, which can be exclusively obtained by high-pressure crystallisation, crystallises in the orthorhombic crystal system in the space group C2221 with a single $\beta$-CD molecule in the asymmetric unit. Unlike all other forms, which crystallise as 1:1 complexes (Table 4.1), this new high-pressure phase contains a lower non-stoichiometric amount of paracetamol. The site occupancy factor of paracetamol refines to a value of $c a$. 0.3 which was later fixed during the refinement. The absence of the guest in the structure is compensated by an extensively disordered water network.

The number of water molecules has been refined to a value of 24.20 per inclusion unit. This is to the best of our knowledge the largest hydration number for an inclusion complex of $\beta$-CD and we refer to this here as "super hydration". With two exceptions, all primary hydroxy groups of $\beta$ $\mathrm{CD}$ are facing the exterior of the cavity, thus exhibit the preferred (-)-gauche conformer (details on how to define the conformation are in Chapter 3); two hydroxy groups were found to be disordered over two positions, with the disordered counterparts exhibiting the (+)-gauche conformer and site occupancies of 0.60 and 0.33 . Both (+)-gauche conformers are stabilised by H- 
bonds to a water molecule inside the cavity. Details about the refinement of the structure and assignment of water molecules are discussed below.

Table 4.1. Crystallographic data of the five forms of $\beta$-CD.paracetamol inclusion complexes

\begin{tabular}{|c|c|c|c|c|c|c|}
\hline & Form $\mathbf{I}^{16}$ & Form Ia & Form II $^{17}$ & Form III ${ }^{17}$ & Form IV ${ }^{17}$ & Form $\mathrm{V}^{\mathrm{a}}$ \\
\hline Formula & $\begin{array}{l}\mathrm{C}_{42} \mathrm{H}_{72} \mathrm{O}_{35}, \\
\mathrm{C}_{8} \mathrm{H}_{9} \mathrm{NO}_{2}, \\
13.3\left(\mathrm{H}_{2} \mathrm{O}\right)\end{array}$ & $\begin{array}{l}\mathrm{C}_{42} \mathrm{H}_{72} \mathrm{O}_{35}, \\
\mathrm{C}_{8} \mathrm{H}_{9} \mathrm{NO}_{2}, \\
8.6(\mathrm{H} 2 \mathrm{O})\end{array}$ & $\begin{array}{c}\mathrm{C}_{42} \mathrm{H}_{72} \mathrm{O}_{35}, \\
\mathrm{C}_{8} \mathrm{H}_{9} \mathrm{NO}_{2}, \\
11.85\left(\mathrm{H}_{2} \mathrm{O}\right)\end{array}$ & $\begin{array}{l}\mathrm{C}_{42} \mathrm{H}_{72} \mathrm{O}_{35}, \\
\mathrm{C}_{8} \mathrm{H}_{9} \mathrm{NO}_{2}, \\
15.2\left(\mathrm{H}_{2} \mathrm{O}\right)\end{array}$ & $\begin{array}{c}\mathrm{C}_{42} \mathrm{H}_{72} \mathrm{O}_{35}, \\
\mathrm{C}_{8} \mathrm{H}_{9} \mathrm{NO}_{2}, \\
n\left(\mathrm{H}_{2} \mathrm{O}\right)\end{array}$ & $\begin{array}{c}\mathrm{C}_{42} \mathrm{H}_{72} \mathrm{O}_{35}, \\
0.3\left(\mathrm{C}_{8} \mathrm{H}_{9} \mathrm{NO}_{2}\right), \\
24.2\left(\mathrm{H}_{2} \mathrm{O}\right)\end{array}$ \\
\hline Space group & C2 & C2 & $P 2{ }_{1}$ & $P 1$ & $P 1$ & C2221 \\
\hline$a / \AA$ & $19.207(7)$ & $18.9343(8)$ & $18.9045(13)$ & $17.9671(13)$ & $15.273(4)$ & $19.6621(11)$ \\
\hline$b / \AA$ & $24.48(1)$ & $24.4136(11)$ & 24.4918(17) & $15.2364(11)$ & $15.576(2)$ & 24.0977(12) \\
\hline$c / \AA ̊$ & $15.700(5)$ & $15.5941(7)$ & $15.7281(12)$ & $15.3783(11)$ & $15.4464(19)$ & $33.071(4)$ \\
\hline$\alpha /{ }^{\circ}$ & 90 & 90 & 90 & $103.240(3)$ & $79.562(9)$ & 90 \\
\hline$\beta /^{\circ}$ & $109.52(29)$ & $110.798(3)$ & $109.186(4)$ & $113.260(3)$ & $74.575(17)$ & 90 \\
\hline$\gamma /{ }^{\circ}$ & 90 & 90 & 90 & $99.323(3)$ & 88.190(17) & 90 \\
\hline$V / \AA^{3}$ & $6959(5)$ & $6738.7(5)$ & $6877.7(9)$ & $3611.8(5)$ & 3483(1) & $15669(2)$ \\
\hline$Z^{\prime}$ & 1 & 1 & 2 & 2 & 2 & 1 \\
\hline$D_{\text {calc }} / \mathrm{g} \mathrm{cm}^{-3}$ & 1.456 & 1.403 & l & 1 & 1 & 1.370 \\
\hline Pressure $^{b}$ & $0.1 \mathrm{MPa}$ & $0.1 \mathrm{MPa}$ & $0.1 \mathrm{MPa}$ & $0.1 \mathrm{MPa}$ & $0.9 \mathrm{GPa}$ & $0.8 \mathrm{GPa}$ \\
\hline$T / \mathrm{K}$ & $298(2)$ & $298(2)$ & $120(2)$ & $120(2)$ & $298(2)$ & $298(2)$ \\
\hline $\begin{array}{l}\text { Meas./uniq./ } \\
\text { obs. Refl.c }\end{array}$ & $\begin{array}{c}6472 / 5103 / \\
2176^{\mathrm{d}}\end{array}$ & $\begin{array}{c}15790 / 5481 / \\
4004\end{array}$ & i & l & i & $\begin{array}{c}58384 / 3328 / \\
2747\end{array}$ \\
\hline Parameters & 458 & 847 & I & 1 & 1 & 1018 \\
\hline Restraints & / & 2365 & / & l & / & 2615 \\
\hline$R_{\text {int }}$ & 0.0302 & 0.0705 & I & 1 & I & 0.0940 \\
\hline$d_{\max } / \AA ̊ \AA$ & l & 1.0 & l & l & I & 1.0 \\
\hline $\begin{array}{c}\text { Complet. to } \\
d_{\max }\end{array}$ & 1 & 0.896 & 1 & 1 & 1 & 0.748 \\
\hline$R_{1}{ }^{e}$ & 0.1345 & 0.0750 & I & I & I & 0.0843 \\
\hline $\begin{array}{c}R_{\text {free }}{ }^{e} \\
\text { (obs. Refl.) f }\end{array}$ & 1 & $\begin{array}{c}0.1012 \\
(605)\end{array}$ & I & 1 & 1 & $\begin{array}{c}0.1285 \\
(330)\end{array}$ \\
\hline$w R_{2} g$ & 1 & 0.1852 & I & I & I & 0.2355 \\
\hline
\end{tabular}

a This work. ${ }^{\mathrm{b}}$ These values refer to the pressure at which diffraction data were collected. ${ }^{\mathrm{c}}$ Criteria for observed reflections: $I$ $>2 \sigma(I) .{ }^{\mathrm{d}}$ Criteria for observed reflections: $I>3 \sigma(I) \cdot{ }^{\mathrm{e}}\left[F^{2}>2 \sigma\left(F^{2}\right)\right] .{ }^{\mathrm{f}} 10 \%$ of unique reflections $\mathrm{g}\left(F^{2}\right)$.

In form $\mathrm{V}$ the inclusion complex crystallises as dimers of $\mathrm{CD}$ molecules, which is the most frequently encountered packing motif for $C D$ molecules in the solid state, as is discussed throughout this chapter. Molecular dynamics calculations performed in vacuum demonstrate that the head-to-head dimer, as encountered in this structure, is the most energetically stable motif. ${ }^{32} \mathrm{~A}$ dimer is formed by two host molecules H-bonded by their secondary hydroxy groups, and is commonly named a head-to-head dimer. Other names for this type of dimer are also found in the literature, such as "basket", 33 which we personally find inaccurate, and "barrel-like motif", ,34 which expresses adequately the shape of the dimer. The barrel-like dimer can be thought as the building block of form V. Dimers stack to form infinite wavy channels when viewed along the [1 1 0] direction (Figure 4.8.a), that can also be described as zigzag or screw channels. Although this is the first time where this packing is observed for $\beta$-CD.paracetamol, it is not unknown for other $\beta$ CD inclusion complexes.

There are two seminal papers devoted to the classification of crystal packing in $\beta-C D$ inclusion complexes by Mentzafos et al..$^{35}$ and Saenger and Steiner. ${ }^{33}$ The classification systems can be combined and are illustrated in Figure 4.7. Saenger and Steiner ${ }^{33}$ tried to come up with uniform 
and widely applicable scheme of the packing of $\beta-\mathrm{CD}$, without focussing on any space group classification, whilst Mentzafos et al.35 were more interested in a classification that takes the incidence of space groups into account.

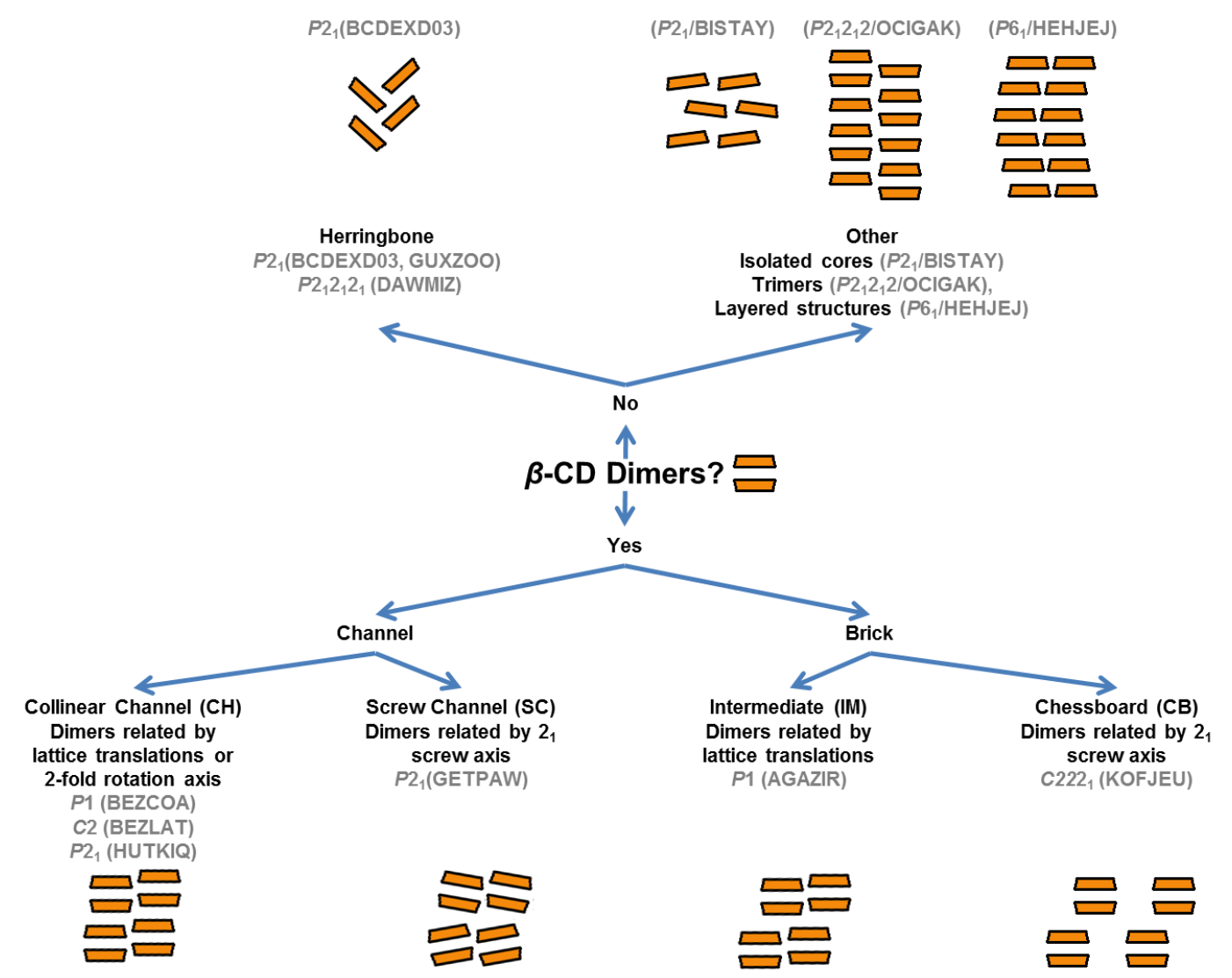

Figure 4.7. Summary and examples of $\beta$-CD packing types in the literature interpreted from references 33,35 .

Mentzafos et al. ${ }^{35}$ previously classified $\beta$-CD inclusion complexes crystallising in space group C2221 and having a unit cell with orthogonal axes of 19, 24 and $32 \AA$ as having a "chessboard" packing motif (CB). The CB motif is formed by adjacent layers of dimers related by a 21-screw axis: "Each dimer is located above the interdimer space of the dimeric layer underneath", i.e. when one layer is seen along the $a$-axis as in Figure 4.8.b. The packing of $\beta$-CD inclusion complexes has also been described as "wave-like polymeric supermolecules". ${ }^{36}$ Mentzafos et al. also described a screw-channel (SC) packing type characteristic of $P 2{ }_{1}$ structures, in which dimers are stacked above each other to form a channel with a screw-like appearance (Figure 4.8.a). The same authors further ascribed the presence of dimers in these structures to pseudo-centred dimeric layers, i.e. the $P 21$ unit cells can be transformed to pseudo C-centred lattices similar to the C2221 type. Mentzafos et al. also reported that there is almost no superposition of the projection of two adjacent dimers related by the $2{ }_{1}$ screw axis in $C 2221$ structures whereas the ones of $P 21$ structures partially overlap. ${ }^{35}$ From our work, the 
structure of $\beta$-CD.paracetamol form $V$ appears to be equally describable by either the CB or the SC packing features (Figure 4.8.a). This might be an indication of similarities between both CB and SC packing types, which is investigated in greater detail in the following section.

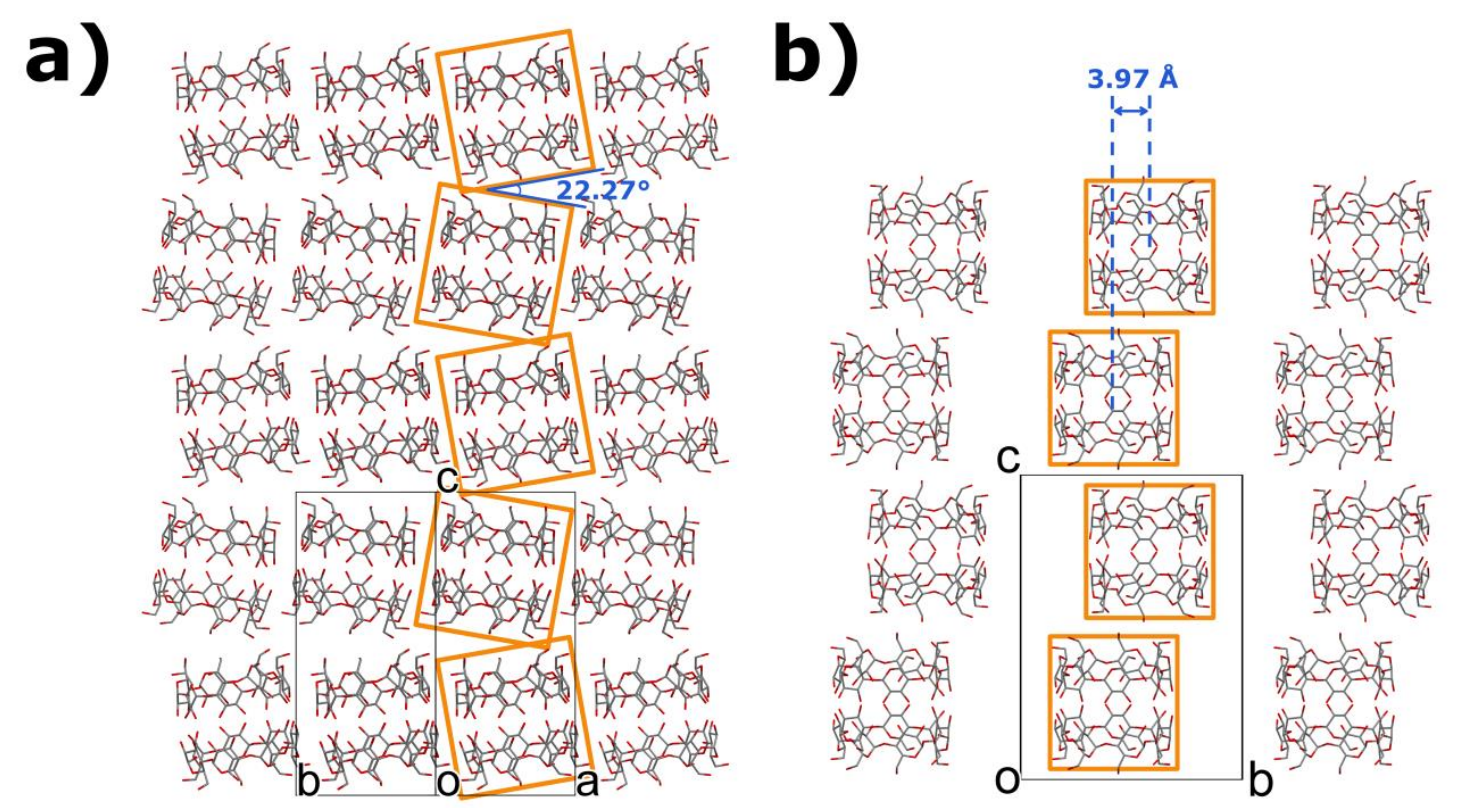

Figure 4.8. Packing feature of $\beta$-CD paracetamol form V inclusion complex. (a) seen along the $a$-axis (CB motif); (b) seen along the $\left[\begin{array}{lll}1 & 1 & 0\end{array}\right]$ direction (SC motif). H atoms, guest and water molecules have been omitted for clarity.

\subsubsection{Crystal packing similarities between $C 222_{1}$ and $P 21$ structures}

In order to better investigate the packing mode of $\beta$-CD.paracetamol form $\mathrm{V}$ and identify packing similarities with structurally related inclusion complexes, a CSD (V. 5.36 including updates to May 2015) study was carried out. For the search in CONQUEST, ${ }^{37}$ the values of the reduced unit cell parameters of form $\mathrm{V}$ were chosen, using a tolerance of $5 \%$ based on the length of the longest axis. 37 hits (excluding 2 redeterminations) corresponding to structures of $\beta$-CD inclusion complexes reported in $P 1, C 2, P 21$ and $C 2221$ space groups (Table 4.2) were identified. The majority of the structures belong to either $P 21$ or $C 222_{1}$ space groups, with 16 and 14 hits, respectively. These structures crystallise in either the $\mathrm{SC}$ or $\mathrm{CB}$ packing types, respectively. $P 1$ and $C 2$ structures, in which $\beta$-CD dimers pack in different modes, were not taken into account for the analysis.

In the following discussion individual structures are referred to by their CSD refcodes, and structural groups by the space group. For reasons discussed below, our $\beta$-CD paracetamol form $\mathrm{V}$ structure is distinct from the $C 222_{1}$ and $P 21$ groups. 
The first comparison of $P 21$ and $C 222_{1}$ structures focuses on the metric symmetry of the system: space group $P 21$ is a subgroup of $C 2221$. Hence, the $C 2221$ lattice can be easily transformed to a $P 21$ one using the transformation matrix:

$$
\left(\begin{array}{ccc}
0.5 & -0.5 & 0 \\
0 & 0 & -1 \\
0.5 & 0.5 & 0
\end{array}\right)
$$

Conversion of a $P 21$ unit cell to an orthorhombic $C 2221$ supercell, by using the inverse of the matrix (I.2.1), is only possible if $(a=c)$ (Figure 4.9.b). All $P 21$ structures have $(a \approx c)$ but only one $(\mathrm{KIFPAQ})^{38}$ strictly fulfils the rule $(a=c)$ and can be transformed to the $C 2221$ space group. Moreover, from the $C 2221 \rightarrow P 21$ transformation matrix it is noticeable that the $a$ - and $c$-axes of the monoclinic unit cell correspond to the $\left[\begin{array}{lll}1 & -1 & 0\end{array}\right]$ and $\left[\begin{array}{lll}1 & 1 & 0\end{array}\right]$ directions of the orthorhombic cell, respectively. Hence, screw channels reported in the $P 21$ structural group and viewed along the $a$ - or $c$-axis can also be seen in the $C 2221$ structural group when viewing the packing along [1 - 10$]$ and [1 10 ] direction, though the degree of displacement of dimers is much greater and is ultimately responsible for grouping these structures in another group ( $\mathrm{CB}$, brick type). In other words, $\mathrm{CB}$ structures can also be described as SC structures if the direction of view is other than the unit cell directions.
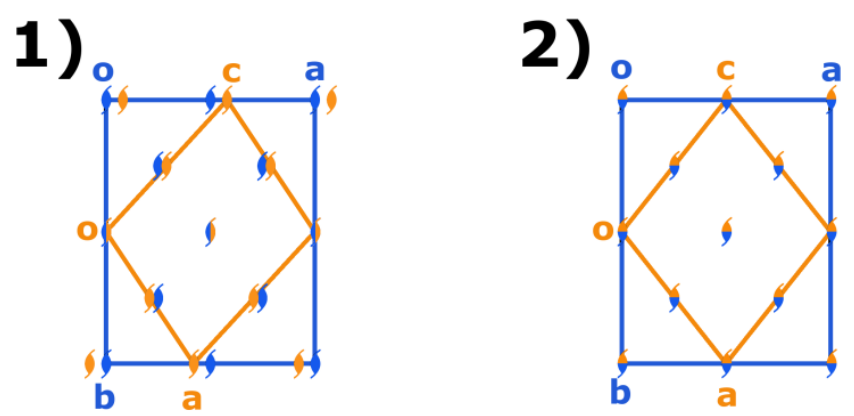

$$
\begin{aligned}
& \mathbf{a}=a+c \\
& \mathbf{b}=a-c \\
& \mathbf{c}=-\mathbf{b}
\end{aligned}
$$

Figure 4.9. Relationship between monoclinic (orange, seen along $b$ ) and orthorhombic (blue, seen along $c$ ) unit cells. (1) $\mathrm{a} \neq \mathrm{c}$ in the monoclinic cell, hence 21 -screw axes in the orthorhombic and monoclinic unit cells mismatch; (2) monoclinic $a=c$ and 21 -screw axes of both unit cells overlap perfectly.

The Crystal Packing Similarities plugin available in MERCURY ${ }^{39}$ was used to further confirm the similarities between $P 2{ }_{1}$ and $C 222{ }_{1}$ structures. The $\beta$-CD backbone was used as comparison motif: all guest and solvent molecules, as well as hydroxy groups of $\beta$-CD were omitted in all structures prior to the comparison. Crystal Packing Similarities compares each pair of structures within a defined coordination shell, and outputs the number of overlaying molecules, the relative root mean square difference (RMSD) and powder X-ray diffraction (PXRD) similarity values.

A first calculation on a single dimer (two $\beta$-CD molecules) shows that although the dimer is formed by symmetry related molecules in the $C 2221$ structural group, the one found in the $P 21$ 
group is related by a pseudo-symmetry element (a 2-fold rotation axis). The analysis shows that in all structures the dimers are practically indistinguishable. They have root mean square deviations (RMSD) values varying between 0.03 and $0.3 \AA$ (see 4.7.Appendix - Table 4.5). The high values of the range are mainly caused by the CDETAN structure: bond lengths and angles in this structure $(R$-factor $\approx 0.25)$ appear chemically incorrect although the structure was nicely described by Tokuoka et al. ${ }^{40}$ (a geometry check using MOGUL ${ }^{41}$ shows a high number of unusual bond lengths and angles). This analysis suggests that the two $\beta$-CD backbones within the frequently observed $\beta$ $\mathrm{CD}$ dimeric arrangement could be, at least in the case of $P 21$ structures, associated by a local 2-fold rotation.

Subsequent crystal packing similarities calculations using a coordination shell of 30 molecules, geometric tolerances of $30 \%$ on the interatomic distances and of $30^{\circ}$ on the interatomic angles, were carried out. We discovered that the LACTAL structure, ${ }^{42}$ although reported as $\beta$-CD by the authors, actually has the wrong handness. 3D coordinates in LACTAL have been inverted prior to the calculations. The results of Crystal Packing Similarities, listed in Table 4.3, show the following points:

1) Within the same structural group, all $C 222_{1}$ structures, and all but two (KIFPAQ, BEGWEQ) of the $P 2{ }_{1}$ structures overlay perfectly with each other, but the overlay between both groups is not as good. This confirms these are two distinct packing types;

2) All powder X-ray diffraction patterns are at least $97 \%$ similar (See Appendix - Table 4.6);

3) KIFPAQ is an outlier in the $P 2{ }_{1}$ structural group and fits nicely in the $C 222{ }_{1}$ group, which is in line with its observed higher symmetry. This strongly suggest the reported low-symmetry space group is incorrect;

4) RMSD values in the C2221 structural group are in the range 0.092-0.398 $\AA$ and are lower than those of $P 21$ structures $0.109-2.132 \AA$, with KIFPAQ here treated as $C 2221$ structure;

5) RMSD values comparing C222 $2_{1}$ vs $P 2{ }_{1}$ structures are in the range $2.795-3.313 \AA$ expressing larger packing differences;

6) Although $\beta$-CD.paracetamol form $\mathrm{V}$ is a $\mathrm{C} 222{ }_{1}$ structure having a SC packing motif, the RMSD table (Table 4.3) shows that its packing is different from either that of both $C 222_{1}$ and $P 21$ structures, but overall when considering the number of molecules in the cluster for comparison (top row of table 3), form V structure fits better in the C2221 structural group 
7) In the $P 2{ }_{1}$ structural group, BEGWEQ ${ }^{43}$ deviates more from the other $P 2{ }_{1}$ structures, and appears to be an outlier in the $P 21$ group. This is investigated in more details below.

In order to complement the analysis of the crystal packing similarities, visual inspection of the structures in MERCURY and computation of packing parameters have been carried out. To simplify the calculations, $\beta$-CD backbone was substituted by the respective centroid of the seven glycosidic $\mathrm{O}(4)$ atoms. The packing features of the SC structural group were defined in the $P 21$ setting using the following parameters: the inner spacing of the channel seen along the monoclinic $b$-axis (i.e. c-axis in C2221), which corresponds to the distance between the projections of the centroids of non-symmetry related molecules on the ac plane; the same spacing projected onto the $a$ and the $c$-axes; and the angle ( $\Psi$ ) between the planes formed by the SC and the (10-1) plane (i.e. the orthorhombic ac plane). Both parameters are graphically detailed in Figure 4.10 and their values compiled in Table 4.2. An additional parameter corresponding to the opening of an SC channel described by the angle $(\xi)$ between $\mathrm{O}(4)$ planes of two consecutive dimer related by the 21 -screw axis, and reported by Mentzafos et al. of about $20^{\circ}$ is consistent within all structures.

The SC inner spacing and the angular difference $(\Psi)$ with the monoclinic (10-1) plane are driving the large RMSD shifts between $C 2221$ and $P 21$ structures seen in Table 4.3, as these values are related with the general position of the dimer in the asymmetric unit: 1) in C2221 structures the dimer forming the $\mathrm{SC}$ is constrained by a 2 -fold rotation axis to be perpendicular to the monoclinic (10-1) plane (i.e. the orthorhombic ac plane), whilst in the $P 21$ group, the SCs are symmetrically more relaxed, and they form an angular difference varying between 3.7 and $23.7^{\circ}$ with the majority below $9^{\circ}$; 2) the spacing of $C 2221$ SCs, i.e. when structures seen along the monoclinic $b$-axis, is homogeneous throughout all structures within the group with a mean value of $8.5 \AA$. In the $P 21$ structural group, the dimers are closer in space and the mean spacing value is ca. $4 \AA$ : BEGWEG as well as KIFPAQ have highly divergent values and were not included in the calculation of the mean spacing value.

These two packing parameters fully explain the packing differences seen in all C2221 and P21 structures as reported in Table 4.2: the homogenous trend seen in the RMSD values of the C2221 group can be explained by the fact that $C 2221$ structures are affected only by the SC spacing as they are constrained to be directed perpendicularly to the monoclinic (10-1) plane, with a spacing distance varying from 7.6 to $8.8 \AA$. In contrast, although the lower symmetry in the $P 2{ }_{1}$ structural group makes the dimers more relaxed, the spacing of the SC is homogenous and varies from 3.5 to $4.7 \AA$ (BEGWEG and KIFPAQ not included), the angular difference of the SC structural group with the (10-1) plane correlates more closely with the RMSD shifts. 
Table 4.2. $\beta$-CD inclusion complex structures reported in the CSD and similar to $\beta$-CD.paracetamol form V.

\begin{tabular}{|c|c|c|c|c|c|c|c|c|c|c|c|c|c|c|c|c|c|c|}
\hline \multirow{2}{*}{ Refcode } & \multirow{2}{*}{ Year } & \multirow{2}{*}{$R$-factor } & \multirow{2}{*}{ Sp. Gp. ${ }^{a}$} & \multirow{2}{*}{$\begin{array}{l}\mathrm{T} \\
/ \mathrm{K}\end{array}$} & \multirow{2}{*}{$\mathrm{z}^{\prime}$} & \multirow{2}{*}{$\mathrm{z}$} & \multicolumn{7}{|c|}{ Reduced cell parameters } & \multirow{2}{*}{$\begin{array}{l}\Psi^{\mathrm{b}} \\
/^{\circ}\end{array}$} & \multirow{2}{*}{$\begin{array}{c}\text { Inner } \\
\text { spacing }^{c} / \AA ̊\end{array}$} & \multirow{2}{*}{$\begin{array}{l}\text { Spacing } \\
\left(\text { a) }{ }^{d} / \AA \AA\right.\end{array}$} & \multirow{2}{*}{$\begin{array}{l}\text { Spacing } \\
(c)^{d} / \AA \AA\end{array}$} & \multirow{2}{*}{$\begin{array}{l}\xi^{\mathrm{e}} \\
/^{\circ}\end{array}$} \\
\hline & & & & & & & $\mathrm{a} / \AA ̊ \AA$ & $\mathrm{b} / \AA$ & $c / \AA ̊ \Omega$ & $\alpha /^{\circ}$ & $6 /^{\circ}$ & $\mathrm{y} /{ }^{\circ}$ & $V / \AA^{3}$ & & & & & \\
\hline BOSZUF $^{36}$ & 2009 & 0.1077 & $\mathrm{C} 222_{1}$ & 293 & 1 & 8 & 15.36701 & 15.36701 & 32.311 & 90 & 90 & 101.8058 & 7469 & 90 & 7.69 & 5.439 & 5.439 & 19.80 \\
\hline BOTBAO $^{36}$ & 2009 & 0.0860 & $\mathrm{C} 222_{1}$ & 100 & 1 & 8 & 15.31715 & 15.31715 & 32.864 & 90 & 90 & 101.3525 & 7560 & 90 & 7.71 & 5.45 & 5.45 & 20.00 \\
\hline DEVTON $^{44}$ & 1984 & 0.1300 & $\mathrm{C} 222_{1}$ & 295 & 1 & 8 & 15.4869 & 15.4869 & 32.84 & 90 & 90 & 101.5826 & 7716 & \multicolumn{5}{|c|}{$3 \mathrm{D}$ coordinates unavailable } \\
\hline DEVTUT $^{44}$ & 1984 & 0.1200 & $\mathrm{C} 222_{1}$ & 295 & 1 & 8 & 15.43908 & 15.43908 & 32.58 & 90 & 90 & 103.6251 & 7547 & \multicolumn{5}{|c|}{$3 \mathrm{D}$ coordinates unavailable } \\
\hline DUYVID $^{45}$ & 2010 & 0.0512 & $\mathrm{C} 222_{1}$ & 173 & 1 & 8 & 15.38309 & 15.38309 & 32.596 & 90 & 90 & 102.9829 & 7516 & 90 & 8.68 & 5.405 & 5.405 & 19.57 \\
\hline FALDUS $^{28}$ & 2004 & 0.1244 & $\mathrm{C} 222_{1}$ & 293 & 1 & 8 & 15.53346 & 15.53346 & 32.62 & 90 & 90 & 101.4534 & 7714 & 90 & 8.57 & 5.423 & 5.423 & 18.40 \\
\hline FASXUS $^{46}$ & 1985 & 0.1150 & $\mathrm{C} 222_{1}$ & 113 & 1 & 8 & 15.34196 & 15.34196 & 32.597 & 90 & 90 & 102.7096 & 7485 & 90 & 8.47 & 5.292 & 5.292 & 19.30 \\
\hline GIPFEQ $^{47}$ & 1988 & 0.1000 & $\mathrm{C} 222_{1}$ & 295 & 1 & 8 & 15.4718 & 15.4718 & 32.503 & 90 & 90 & 102.2021 & 7605 & 90 & 8.48 & 5.328 & 5.328 & 18.68 \\
\hline KEMZIN $^{48}$ & 2013 & 0.1051 & $\mathrm{C} 222_{1}$ & 100 & 1 & 8 & 15.32311 & 15.32311 & 32.554 & 90 & 90 & 102.3997 & 7465 & 90 & 8.83 & 5.53 & 5.53 & 21.22 \\
\hline KOFJEU $^{35}$ & 1991 & 0.1127 & $\mathrm{C} 222_{1}$ & 295 & 1 & 8 & 15.52019 & 15.52019 & 32.808 & 90 & 90 & 103.5983 & 7681 & 90 & 8.69 & 5.372 & 5.372 & 19.50 \\
\hline LACTAL $^{42}$ & 2004 & 0.1355 & $\mathrm{C} 222_{1}$ & 293 & 1 & 8 & 15.51729 & 15.51729 & 32.806 & 90 & 90 & 102.8701 & 7701 & 90 & 8.70 & 5.423 & 5.423 & 19.45 \\
\hline MEGQUK $^{49}$ & 2000 & 0.1082 & $\mathrm{C} 222_{1}$ & 110 & 1 & 8 & 15.33049 & 15.33049 & 32.67 & 90 & 90 & 102.7271 & 7490 & 90 & 8.71 & 5.437 & 5.437 & 21.16 \\
\hline WEZSIE $^{50}$ & 2007 & 0.1103 & $\mathrm{C} 222_{1}$ & 173 & 1 & 8 & 15.39446 & 15.39446 & 32.471 & 90 & 90 & 103.5959 & 7480 & 90 & 8.52 & 5.272 & 5.272 & 19.45 \\
\hline XUXFAY ${ }^{51}$ & 2009 & 0.0903 & $\mathrm{C} 222_{1}$ & 113 & 1 & 8 & 15.36045 & 15.36045 & 32.4473 & 90 & 90 & 102.699 & 7468 & 90 & 8.79 & 5.492 & 5.492 & 20.47 \\
\hline This Work & 2015 & 0.0742 & $\mathrm{C} 222_{1}$ & 298 & 1 & 8 & 15.5507 & 15.5507 & 33.071 & 90 & 90 & 101.576 & 7834 & 90 & 3.97 & 2.508 & 2.508 & 22.27 \\
\hline BEGWEQ $^{43}$ & 2003 & 0.0662 & $P 2_{1}$ & 173 & 1 & 2 & 15.2262 & 15.6739 & 31.4771 & 90 & 90 & 104.32 & 7279 & 23.65 & 7.01 & 3.232 & 6.829 & 20.88 \\
\hline BOTBES $^{36}$ & 2009 & 0.0662 & $P 2_{1}$ & 100 & 2 & 4 & 15.3151 & 15.3818 & 31.5498 & 90 & 90 & 101.661 & 7279 & 4.22 & 4.15 & 3.396 & 3.023 & 20.24 \\
\hline CIVBUE $^{52}$ & 1984 & 0.1600 & $P 2_{1}$ & 295 & 1 & 2 & 15.229 & 15.562 & 33.189 & 90 & 90 & 104.854 & 7603 & \multicolumn{5}{|c|}{$\begin{array}{l}\text { S.15 } 3.050 \\
\text { 3D coordinates unavailable }\end{array}$} \\
\hline CDETAN $^{40}$ & 1981 & 0.2501 & $P 2_{1}$ & 295 & 2 & 4 & 15.3 & 15.584 & 32.311 & 90 & 90 & 102.4 & 7524 & 8.83 & 3.53 & 2.346 & 3.075 & 17.14 \\
\hline DUTLIN $^{53}$ & 1986 & 0.1210 & $P 2_{1}$ & 128 & 2 & 4 & 15.277 & 15.316 & 32.232 & 90 & 90 & 101.18 & 7399 & \multicolumn{5}{|c|}{ 3D coordinates unavailable } \\
\hline DUTLIN10 ${ }^{54}$ & 1988 & 0.1080 & $P 2_{1}$ & 123 & 2 & 4 & 15.277 & 15.316 & 32.232 & 90 & 90 & 101.18 & 7399 & 6.31 & 3.98 & 3.334 & 2.786 & 20.60 \\
\hline FODBIK $^{55}$ & 2005 & 0.0972 & $P 2_{1}$ & 293 & 2 & 4 & 15.46 & 15.512 & 32.209 & 90 & 90 & 103.096 & 7523 & 6.32 & 4.61 & 3.396 & 3.8 & 19.35 \\
\hline GETPAW $^{56}$ & 1988 & 0.0810 & $P 2_{1}$ & 138 & 1 & 2 & 15.26 & 15.35 & 32.76 & 90 & 90 & 101.5 & 7520 & 8.83 & 4.43 & 3.31 & 3.539 & 21.13 \\
\hline GETPEA $^{56}$ & 1988 & 0.1060 & $P 2_{1}$ & 138 & 1 & 2 & 15.277 & 15.31 & 32.124 & 90 & 90 & 100.76 & 7381 & 5.76 & 3.99 & 3.315 & 2.798 & 19.91 \\
\hline KIFPAQ $^{38}$ & 1999 & 0.1169 & $P 2_{1}$ & 295 & 2 & 4 & 15.428 & 15.437 & 32.545 & 90 & 90 & 103.56 & 7535 & 90 & 8.73 & 5.405 & 5.399 & 19.52 \\
\hline MACDAW $^{34}$ & 2003 & 0.0868 & $P 2_{1}$ & 293 & 2 & 4 & 15.394 & 15.621 & 31.995 & 90 & 90 & 103.738 & 7474 & 7.19 & 3.99 & 3.406 & 2.836 & 18.98 \\
\hline NAJJAK ${ }^{57}$ & 2004 & 0.1163 & $P 2_{1}$ & 200 & 2 & 4 & 15.184 & 15.639 & 32.51 & 90 & 90 & 102.62 & 7533 & 7.43 & 3.98 & 3.364 & 2.812 & 19.75 \\
\hline NAJJAK01 ${ }^{58}$ & 2006 & 0.1163 & $P 2_{1}$ & 200 & 2 & 4 & 15.184 & 15.639 & 32.51 & 90 & 90 & 102.62 & 7533 & \multirow{2}{*}{\multicolumn{5}{|c|}{$\begin{array}{l}\text { 3D coordinates unavailable } \\
\text { 3D coordinates unavailable }\end{array}$}} \\
\hline NIZGUY 59 & 1996 & 0.1080 & $P 2_{1}$ & 295 & 1 & 2 & 15.324 & 15.342 & 32.54001 & 90 & 90 & 102.44 & 7471 & & & & & \\
\hline OKAQAT $^{60}$ & 2011 & 0.1169 & $P 2_{1}$ & 120 & 2 & 4 & 15.3332 & 15.54 & 31.7 & 90 & 90 & 102.459 & 7376 & 3.71 & 4.10 & 3.002 & 3.369 & 19.95 \\
\hline QACXEX $^{61}$ & 1999 & 0.0584 & $P 2_{1}$ & 20 & 2 & 4 & 15.255 & 15.454 & 31.693 & 90 & 90 & 102.92 & 7282 & 5.6 & 4.05 & 2.931 & 3.383 & 19.49 \\
\hline XEYDIQ ${ }^{62}$ & 2012 & 0.1046 & $P 2_{1}$ & 100 & 2 & 4 & 15.2946 & 15.3936 & 32.5293 & 90 & 90 & 102.17 & 7487 & 8.99 & 3.85 & 3.33 & 2.593 & 21.33 \\
\hline YOJHUC ${ }^{63}$ & 2012 & 0.1128 & $P 2_{1}$ & 100 & 2 & 4 & 14.8541 & 15.7645 & 32.3999 & 90 & 90 & 103.75 & 7370 & 11.64 & 3.53 & 2.383 & 3.101 & 20.49 \\
\hline $\mathrm{DEVVEF}^{64}$ & 1984 & 0.2190 & $P 1$ & 295 & 1 & 1 & 15.529 & 15.568 & 32.32715 & 99.8915 & 93.4746 & 103.82 & 7434 & & & & & \\
\hline ASEWAX ${ }^{65}$ & 2011 & 0.1566 & $C_{2}$ & 150 & 0.5 & 2 & 15.38554 & 15.38554 & 33.34401 & 96.0563 & 96.0563 & 104.1396 & 7540 & & & & & \\
\hline EZEYAJ $^{66}$ & 2004 & 0.1032 & $C 2$ & 293 & 2 & 8 & 15.47755 & 15.47755 & 33.457 & 98.5991 & 98.5991 & 102.2555 & 7607 & & & & & \\
\hline HAHVIW $^{67}$ & 2004 & 0.0605 & $C 2$ & 293 & 2 & 8 & 15.34915 & 15.34915 & 32.58 & 92.3404 & 92.3404 & 103.0436 & 7462 & & & & & \\
\hline KOLGAU $^{68}$ & 2008 & 0.0801 & $C 2$ & 298 & 1 & 4 & 15.55352 & 15.55352 & 32.9754 & 98.9353 & 98.9353 & 102.7705 & 7535 & & & & & \\
\hline MACCUP $^{34}$ & 2003 & 0.0826 & $C 2$ & 293 & 2 & 8 & 15.47886 & 15.47886 & 33.315 & 98.6366 & 98.6366 & 102.7758 & 7556 & & & & & \\
\hline WANMII $^{69}$ & 2005 & 0.1021 & $C 2$ & 293 & 0.5 & 2 & 15.45985 & 15.45985 & 32.776 & 92.4825 & 92.4825 & 102.3829 & 7633 & & & & & \\
\hline
\end{tabular}

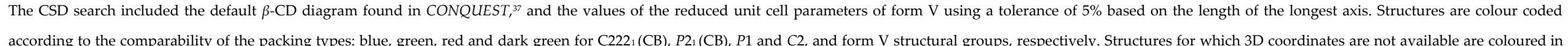

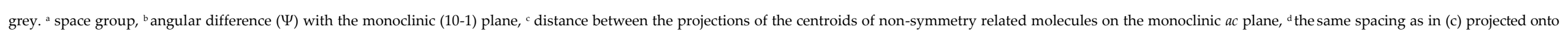
the $a$ and the $c$-axes, ${ }^{e}$ angular difference $(\xi)$ between $\mathrm{O}(4)$ planes of two consecutive dimers. All these parameters are further detailed in Figure 4.10. 
Table 4.3. RMSD values ( $(\AA)$ and maximum number of fitted molecules up to a maximum number of 30 molecules in a cluster, calculated using Crystal Packing Similarities in MERCURY. ${ }^{39}$

\begin{tabular}{|c|c|c|c|c|c|c|c|c|c|c|c|c|c|c|c|c|c|c|c|c|c|c|c|c|c|c|c|}
\hline & \multicolumn{13}{|c|}{$\mathrm{C} 222_{1}$} & \multicolumn{14}{|c|}{$P 2_{1}$} \\
\hline & 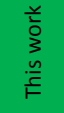 & $\begin{array}{l}\text { 岕 } \\
\widetilde{\infty} \\
\text { O }\end{array}$ & 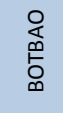 & 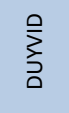 & 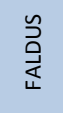 & 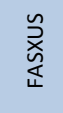 & $\begin{array}{l}\stackrel{g}{u} \\
\text { 产 } \\
\text { 足 }\end{array}$ & 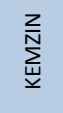 & 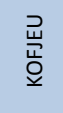 & $\overrightarrow{\vec{E}}$ & $\begin{array}{l}\text { 总 } \\
\text { 崖 }\end{array}$ & $\begin{array}{l}\frac{w}{\bar{N}} \\
\frac{\mathrm{W}}{3}\end{array}$ & 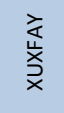 & $\begin{array}{l}\text { 岕 } \\
\text { 岕 } \\
\text { d }\end{array}$ & 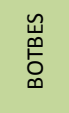 & 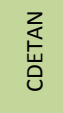 & 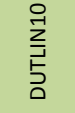 & $\begin{array}{l}\text { 䒑. } \\
\text { ồ }\end{array}$ & $\begin{array}{l}3 \\
\text { 充 } \\
\text { 岕 }\end{array}$ & $\begin{array}{l}\text { 岕 } \\
\text { 岕 }\end{array}$ & $\begin{array}{l}\frac{0}{\alpha} \\
\frac{\alpha}{\underline{\underline{u}}} \\
\end{array}$ & 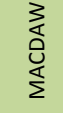 & $\frac{.}{\frac{1}{2}}$ & 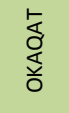 & 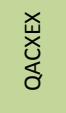 & 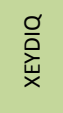 & $\begin{array}{l}\text { ญ } \\
\text { 퐁 }\end{array}$ \\
\hline This work & & 26 & 26 & 26 & 26 & 26 & 26 & 27 & 26 & 26 & 26 & 26 & 26 & 20 & 20 & 20 & 20 & 27 & 27 & 20 & 26 & 20 & 20 & 20 & 20 & 20 & 20 \\
\hline BOSZUF $^{36}$ & 2.30 & & 30 & 30 & 30 & 30 & 30 & 30 & 30 & 30 & 30 & 30 & 30 & 26 & 26 & 20 & 26 & 28 & 28 & 26 & 30 & 26 & 26 & 26 & 26 & 26 & 26 \\
\hline ВОТВАO ${ }^{36}$ & 2.32 & 0.25 & & 30 & 30 & 30 & 30 & 30 & 30 & 30 & 30 & 30 & 30 & 26 & 26 & 20 & 26 & 28 & 28 & 26 & 30 & 26 & 26 & 26 & 26 & 26 & 26 \\
\hline DUYVID ${ }^{45}$ & 2.27 & 0.19 & 0.29 & & 30 & 30 & 30 & 30 & 30 & 30 & 30 & 30 & 30 & 26 & 26 & 20 & 26 & 28 & 28 & 26 & 30 & 26 & 26 & 26 & 26 & 26 & 26 \\
\hline FALDUS $^{28}$ & 2.23 & 0.23 & 0.33 & 0.31 & & 30 & 30 & 30 & 30 & 30 & 30 & 30 & 30 & 26 & 26 & 20 & 26 & 28 & 28 & 26 & 30 & 26 & 26 & 26 & 26 & 26 & 26 \\
\hline FASXUS ${ }^{46}$ & 2.21 & 0.20 & 0.29 & 0.10 & 0.31 & & 30 & 30 & 30 & 30 & 30 & 30 & 30 & 26 & 26 & 20 & 26 & 28 & 28 & 26 & 30 & 26 & 26 & 26 & 26 & 26 & 26 \\
\hline GIPFEQ $^{47}$ & 2.16 & 0.19 & 0.32 & 0.20 & 0.18 & 0.19 & & 30 & 30 & 30 & 30 & 30 & 30 & 26 & 26 & 20 & 26 & 28 & 28 & 26 & 30 & 26 & 26 & 26 & 26 & 26 & 26 \\
\hline KEMZIN ${ }^{48}$ & 2.38 & 0.17 & 0.26 & 0.16 & 0.33 & 0.21 & 0.29 & & 30 & 30 & 30 & 30 & 30 & 27 & 27 & 20 & 27 & 28 & 28 & 27 & 30 & 27 & 27 & 27 & 27 & 27 & 27 \\
\hline KOFJEU ${ }^{35}$ & 2.21 & 0.32 & 0.39 & 0.18 & 0.33 & 0.21 & 0.23 & 0.31 & & 30 & 30 & 30 & 30 & 26 & 26 & 20 & 26 & 28 & 28 & 26 & 30 & 26 & 26 & 26 & 26 & 26 & 26 \\
\hline LACTAL $^{42}$ & 2.22 & 0.26 & 0.33 & 0.17 & 0.23 & 0.20 & 0.16 & 0.27 & 0.12 & & 30 & 30 & 30 & 26 & 26 & 20 & 26 & 28 & 28 & 26 & 30 & 26 & 26 & 26 & 26 & 26 & 26 \\
\hline MEGQUK $^{49}$ & 2.31 & 0.19 & 0.25 & 0.09 & 0.33 & 0.15 & 0.25 & 0.10 & 0.24 & 0.22 & & 30 & 30 & 26 & 26 & 20 & 26 & 28 & 28 & 26 & 30 & 26 & 26 & 26 & 26 & 26 & 26 \\
\hline WEZSIE $^{50}$ & 2.18 & 0.28 & 0.40 & 0.14 & 0.38 & 0.15 & 0.23 & 0.28 & 0.17 & 0.22 & 0.21 & & 30 & 26 & 26 & 20 & 26 & 28 & 28 & 26 & 30 & 26 & 26 & 26 & 26 & 26 & 26 \\
\hline XUXFAY ${ }^{51}$ & 2.35 & 0.16 & 0.27 & 0.11 & 0.32 & 0.18 & 0.25 & 0.09 & 0.26 & 0.24 & 0.10 & 0.22 & & 26 & 26 & 20 & 26 & 28 & 28 & 26 & 30 & 26 & 26 & 26 & 26 & 26 & 26 \\
\hline BEGWEQ $^{43}$ & 2.66 & 3.18 & 3.18 & 3.22 & 3.23 & 3.28 & 3.29 & 3.20 & 3.28 & 3.25 & 3.18 & 3.31 & 3.16 & & 22 & 20 & 20 & 28 & 26 & 20 & 26 & 22 & 22 & 22 & 22 & 20 & 20 \\
\hline BOTBES ${ }^{36}$ & 2.41 & 2.99 & 2.99 & 3.03 & 3.00 & 3.06 & 3.05 & 3.02 & 3.08 & 3.05 & 3.01 & 3.09 & 2.99 & 1.79 & & 30 & 30 & 30 & 30 & 30 & 26 & 30 & 30 & 30 & 30 & 30 & 30 \\
\hline CDETAN ${ }^{40}$ & 2.36 & 2.83 & 2.83 & 2.82 & 2.87 & 2.84 & 2.87 & 2.80 & 2.85 & 2.84 & 2.81 & 2.86 & 2.80 & 2.00 & 0.60 & & 30 & 30 & 30 & 30 & 20 & 30 & 30 & 30 & 30 & 30 & 30 \\
\hline DUTLIN $10^{54}$ & 2.39 & 3.05 & 3.04 & 3.08 & 3.06 & 3.10 & 3.11 & 3.08 & 3.13 & 3.09 & 3.06 & 3.13 & 3.04 & 1.53 & 0.27 & 0.53 & & 30 & 30 & 30 & 26 & 30 & 30 & 30 & 30 & 30 & 30 \\
\hline FODBIK $K^{55}$ & 2.92 & 3.05 & 3.06 & 3.05 & 3.10 & 3.09 & 3.11 & 3.00 & 3.09 & 3.08 & 3.03 & 3.09 & 3.02 & 1.99 & 0.33 & 0.48 & 0.32 & & 30 & 30 & 28 & 30 & 30 & 30 & 30 & 30 & 30 \\
\hline GETPAW $^{56}$ & 2.84 & 3.12 & 3.11 & 3.13 & 3.16 & 3.16 & 3.18 & 3.08 & 3.17 & 3.16 & 3.10 & 3.17 & 3.09 & 2.13 & 0.45 & 0.53 & 0.22 & 0.38 & & 30 & 28 & 30 & 30 & 30 & 30 & 30 & 30 \\
\hline GETPEA $^{56}$ & 2.07 & 3.09 & 3.07 & 3.11 & 3.10 & 3.14 & 3.15 & 3.12 & 3.18 & 3.13 & 3.10 & 3.17 & 3.08 & 1.55 & 0.28 & 0.55 & 0.11 & 0.39 & 0.28 & & 26 & 30 & 30 & 30 & 30 & 30 & 30 \\
\hline $\mathrm{KIFPAQ}^{38}$ & 2.27 & 0.26 & 0.37 & 0.10 & 0.35 & 0.16 & 0.24 & 0.23 & 0.14 & 0.18 & 0.17 & 0.11 & 0.17 & 3.23 & 3.05 & 2.82 & 3.10 & 3.05 & 3.13 & 3.13 & & 26 & 26 & 26 & 26 & 26 & 26 \\
\hline MACDAW $^{34}$ & 2.48 & 2.93 & 2.94 & 2.96 & 2.92 & 3.00 & 2.98 & 2.97 & 2.99 & 2.96 & 2.95 & 3.04 & 2.93 & 1.80 & 0.37 & 0.55 & 0.43 & 0.16 & 0.48 & 0.49 & 2.98 & & 30 & 30 & 30 & 30 & 30 \\
\hline NAJJAK $^{57}$ & 2.41 & 2.96 & 2.95 & 2.99 & 2.95 & 3.01 & 3.00 & 3.00 & 3.02 & 2.99 & 2.97 & 3.04 & 2.96 & 1.85 & 0.44 & 0.59 & 0.35 & 0.28 & 0.31 & 0.43 & 3.00 & 0.30 & & 30 & 30 & 30 & 30 \\
\hline OKAQAT ${ }^{60}$ & 2.45 & 3.03 & 3.04 & 3.06 & 3.04 & 3.09 & 3.10 & 3.06 & 3.11 & 3.08 & 3.04 & 3.12 & 3.02 & 1.80 & 0.22 & 0.53 & 0.35 & 0.27 & 0.50 & 0.37 & 3.08 & 0.34 & 0.50 & & 30 & 30 & 30 \\
\hline QACXEX $^{61}$ & 2.42 & 2.92 & 2.93 & 2.97 & 2.93 & 3.00 & 2.98 & 2.97 & 3.00 & 2.97 & 2.95 & 3.03 & 2.93 & 1.78 & 0.22 & 0.57 & 0.35 & 0.24 & 0.47 & 0.40 & 2.99 & 0.21 & 0.36 & 0.26 & & 30 & 30 \\
\hline XEYDIQ ${ }^{62}$ & 2.41 & 3.04 & 3.02 & 3.05 & 3.03 & 3.07 & 3.08 & 3.08 & 3.11 & 3.06 & 3.04 & 3.10 & 3.02 & 1.85 & 0.41 & 0.49 & 0.22 & 0.27 & 0.14 & 0.30 & 3.07 & 0.37 & 0.25 & 0.42 & 0.38 & & 30 \\
\hline YOJHUC ${ }^{63}$ & 2.37 & 2.99 & 2.99 & 3.01 & 2.99 & 3.03 & 3.03 & 3.05 & 3.05 & 3.01 & 3.01 & 3.06 & 2.99 & 1.94 & 0.70 & 0.76 & 0.65 & 0.56 & 0.58 & 0.72 & 3.03 & 0.49 & 0.39 & 0.76 & 0.56 & 0.52 & \\
\hline
\end{tabular}

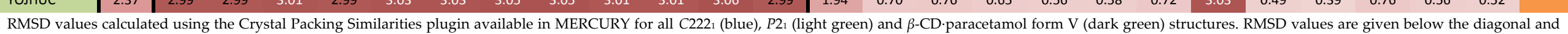
the number of molecules compared is given above the diagonal. Values are colour coded from light to dark according to a decreasing quality of matching. RMSD values have been rounded up to the second decimal. 

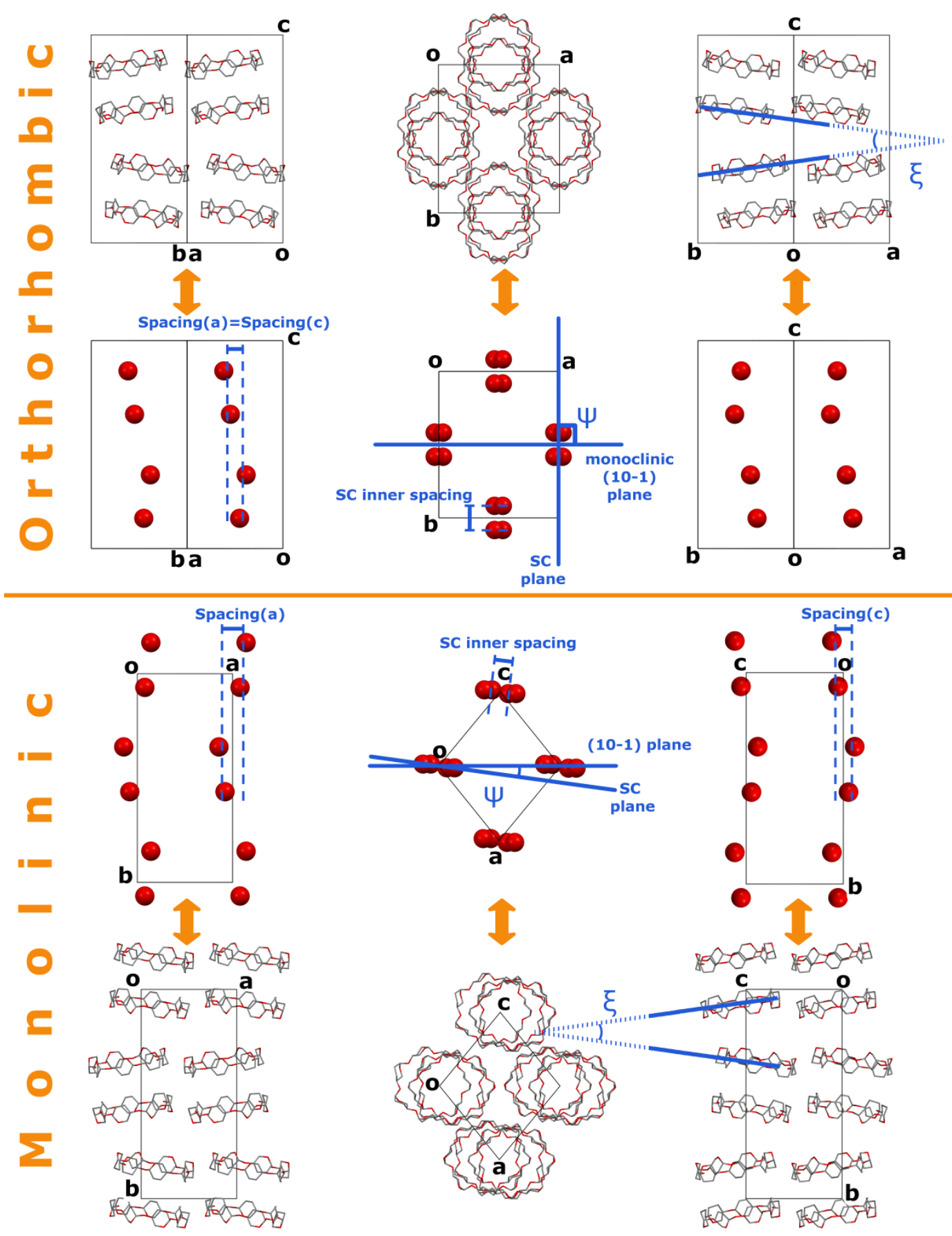

Figure 4.10. Packing parameters describing $C 2221$ and $P 2{ }_{1}$ structural groups. All interpretations have been made in the monoclinic setting. $\beta$-CD paracetamol and GETPAW structures were used to draw the packing parameters for the orthorhombic and monoclinic settings, respectively. Each $\beta$-CD backbone has been substituted by the centroid of the seven glycosidic $\mathrm{O}(4)$ atoms to facilitate the calculations. The packing parameters have been defined as follows: the SC inner spacing is the distance between $P 21$ related dimers, spacing (c) and (a) are the projection of the inner spacing on the c- and a-axes, respectively; $\Psi$ is the angle formed between the SC plane and the monoclinic (10-1) plane; $\xi$ is the angle between $P 21$ related dimers, projected on the a-axis $(\xi \mathrm{a})$ or on the $\mathrm{c}$-axis $(\xi \mathrm{c})$. H atoms, guest and water molecules have been omitted for clarity. 
The packing of BEGWEQ and KIFPAQ was reported to be of the SC mode, although both structures are not isostructural with $\beta-\mathrm{CD}$ dimeric, screw-channel $P 2{ }_{1}$ structures. ${ }^{34,43,70}$ Giastas et al. stated that both structures can be considered as pseudo-CB structures if the guest's symmetry is not taken into account. ${ }^{34}$ In this work, BEGWEQ is considered as an atypical SC P21 structure on the grounds of a SC spacing comparable to that found in C2221 structures, and of the largest angular difference with the monoclinic (10-1) plane in the $P 2{ }_{1}$ pool. BEGWEQ has been nicely described by Caira et al. as an "elongated SC structure". ${ }^{43}$ All values describing the P21 structure of KIFPAQ confirm that it belongs in fact to the higher symmetry space group $C 2221$, where the adamantanone guest molecule would be disordered over a two-fold rotation axis. The structure of KIFPAQ can be correlated to the recently published DUYVID structure of $\beta$-CD-adamantan inclusion complex reported in space group $C 2221 .{ }^{45}$

The screw channel in the structure of $\beta$-CD.paracetamol form $\mathrm{V}$ has the geometrical constraints of $\mathrm{CB} C 222_{1}$ structures but a spacing comparable to SC $P 21$ structures, explaining the homogeneous but high RMSD values with $P 21$ and C2221 structures. Hence, $\beta$-CD.paracetamol form V structure is neither isostructural with the $C 2221$ nor with $P 2{ }_{1}$ structural groups but is best described as an intermediate between the two.
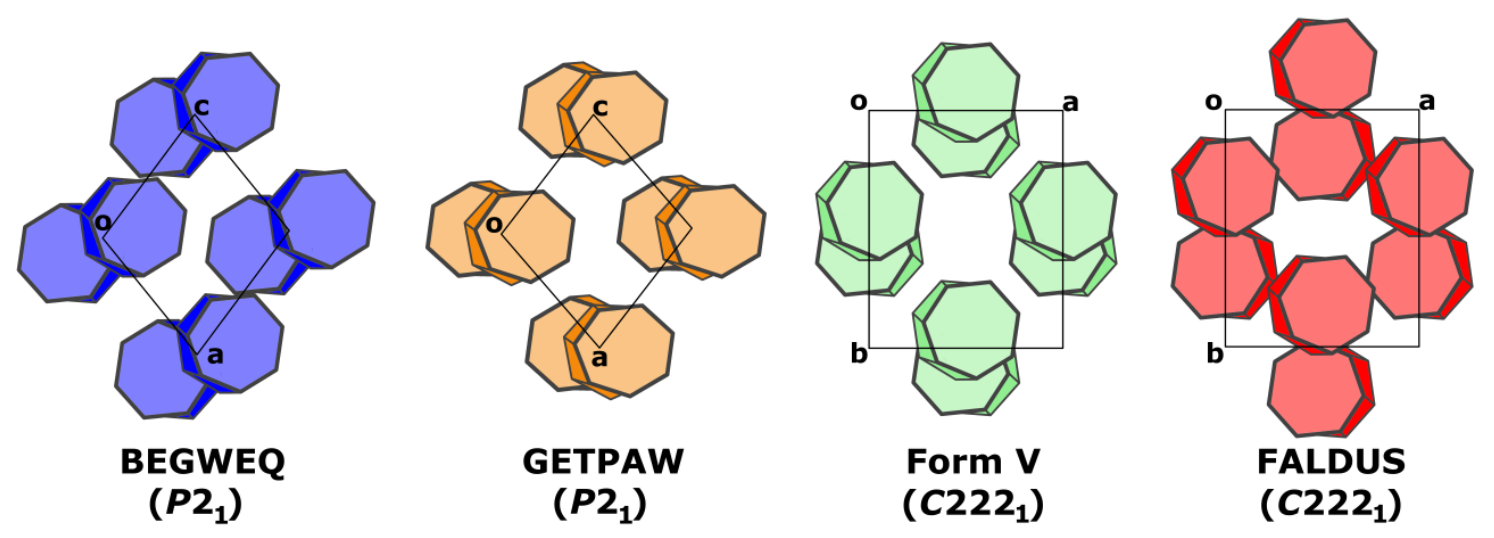

Figure 4.11. Comparison between $C 2221, P 21$ and $\beta$-CD paracetamol form $\mathrm{V}$ packing types. $\beta$-CD dimers represented by the volume delimited by the $14 \mathrm{O}(4)$ atoms. The superposition of the dimers shows the $21^{-}$ screw relationship.

Several authors relate the packing differences in the dimeric $\beta$-CD structures to the shape, size and nature of guest molecules.34,36,43,71,72 Giastas et al. attempted to hypothesise an association of $\beta$-CD packing type with the type of functional group(s) of the guest emerging from the primary faces of the barrel. The hypothesis is based on the contact of the functional groups of the guest with the interstitial aqueous environment: a hydrophilic or a hydrophobic group favours a CB or a SC type packing, respectively. However, it is difficult to generalise the influence of the guest on the packing mode in all $\beta$-CD inclusion complex structures. ${ }^{34}$ In the case of $\beta$-CD.paracetamol form $\mathrm{V}$, on one hand, although the hydroxy group of paracetamol molecules are on the primary opening of 
the dimer, the SC mode is favoured over a true CB packing. On the other hand, the guest stoichiometry is so low that the packing cannot be fully related to the presence of guest molecules. This new distinct packing may be associated with a pressure-driven phenomenon of super hydration of $\beta$-CD molecules, although in the absence of further examples this is only a speculation.

\subsubsection{Structural comparison with form I}

The structure of $\beta$-CD.paracetamol inclusion complex form I was reported by Caira and Dodds in $2000 .{ }^{16}$ The complex is reported to crystallise in the C2 space group and exhibits a collinear channel $(\mathrm{CH})$-type structure (Figure 4.7$)$ in which $\mathrm{CD}$ molecules are arranged as dimers. The lack of 3D coordinates in the CSD for this structure prompted us to redetermine its structure.

When compared to form $\mathrm{I}$, the overall water content per $\beta$-CD unit in form $\mathrm{V}$ almost doubles, increasing from a refined value of 12.3 water molecules per $\beta$-CD (13.3 measured by thermogravimetry $)^{16}$ for form $\mathrm{I}$ at ambient conditions to a value of 24.20 for form $\mathrm{V}$ at highpressure conditions. In form I, only two water molecules are fully ordered in an extended disordered solvent region which is present outside the channels, and only one water molecules is disordered over two positions inside the channels.

At ambient conditions $\beta$-CD.paracetamol crystallises as a 1:1 dimeric complex stacked in channels parallel to the $c$-axis, ${ }^{16}$ a well-known packing motif for similar $C 2$ structures, ${ }^{35}$ whereas the complex in form $\mathrm{V}$ crystallises as an intermediate SC-CB structural motif at high-pressure conditions with a stoichiometry well below unity for the guest molecule. The conservation of the dimeric arrangement in form I and V was checked using the Crystal Packing Similarities plugin in MERCURY: both dimers are similar within an RMSD value of $0.16 \AA$ when $\beta$-CD backbone is compared, or $0.19 \AA$ when the whole $\beta$-CD molecule is compared excluding $\mathrm{O}(6)$ hydroxy groups, which are disordered. At ambient conditions the guest molecules exhibit disorder occupying two positions with equal site occupancy. At high-pressure conditions paracetamol is, as far as the data can tell, ordered with site occupancy of 0.3. The inclination of the guest molecule inside the macrocycle is consistent in both structures: in form I, the values of the angle formed between the glycosidic $\mathrm{O}(4)$ ring and the phenyl ring are 70.5 and $72.6^{\circ}$ for form $\mathrm{I}$, and $74.7^{\circ}$ in form $\mathrm{V}$. However the conformation of the guest differs: in form I, the acetamide end of the paracetamol molecule forms a relatively small dihedral angle with the plane of the phenyl ring $\left(2.6^{\circ}\right.$ and $\left.5^{\circ}\right)$, whereas in form $\mathrm{V}$ the same angular difference is $58^{\circ}$; this value, although high, is still tolerable in the case of form $\mathrm{V}$ where the acetamide group is involved in short $\mathrm{H}$-bonds with water molecules inside the cavity (Figure 4.12). A CSD search reveals that this angular difference is between the acetamide and the phenyl ring is not unusual for paracetamol molecules: with the angle varying between 3 
and $55^{\circ}$ in a total of 83 hits with an average of $21^{\circ}$ (the CSD, V. 5.36 including updates to May 2015 was searched for structure with 3-D coordinates).

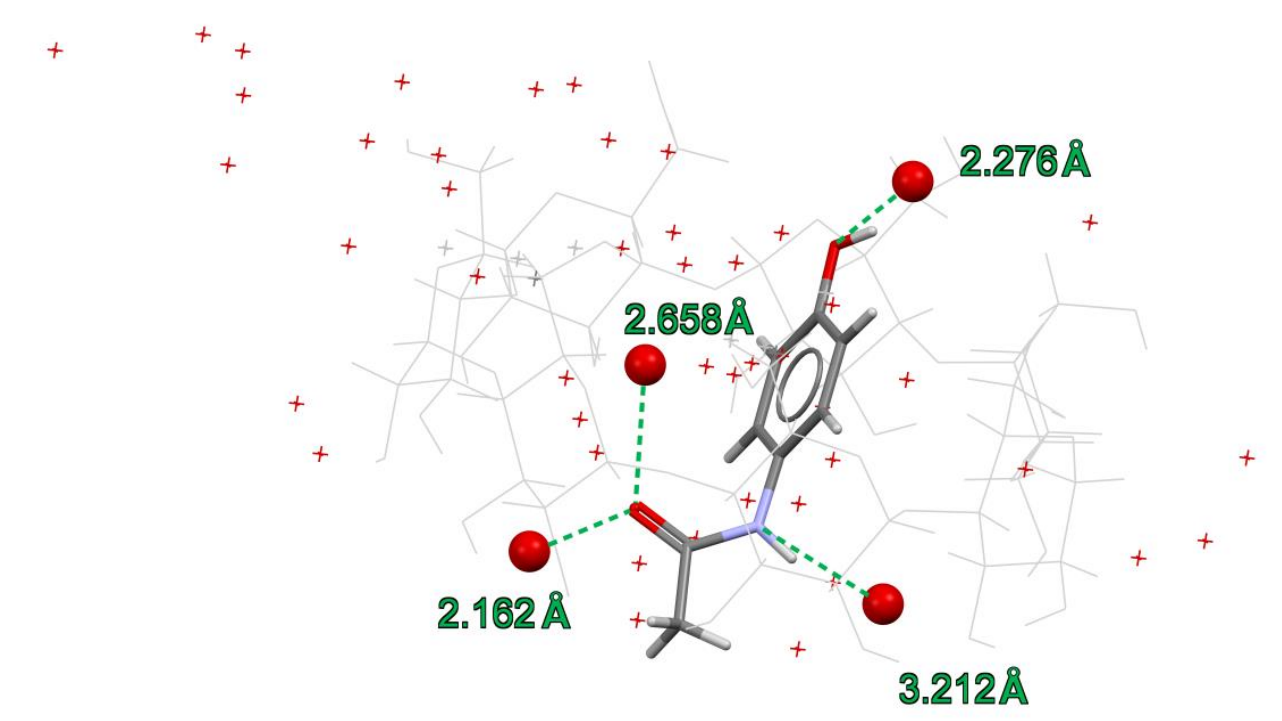

Figure 4.12. $\mathrm{H}$ bonds formed by paracetamol and water molecules inside $\beta$-CD cavity. $\mathrm{H}$ atoms have been omitted for clarity.

\subsubsection{Refinement and modelling of the structure of form $\mathrm{V}$}

\subsubsection{Structure solution and refinement (early stage)}

Structure solution by isomorphous replacement using similar structures reported in the C2221 space group and subsequent structure refinement was unsuccessful. Space group assignment was investigated further. From the diffraction frames there was no indication of split reflections. Twinning through pseudo-merohedry using a smaller non-centered monoclinic $P 2{ }_{1}$ unit

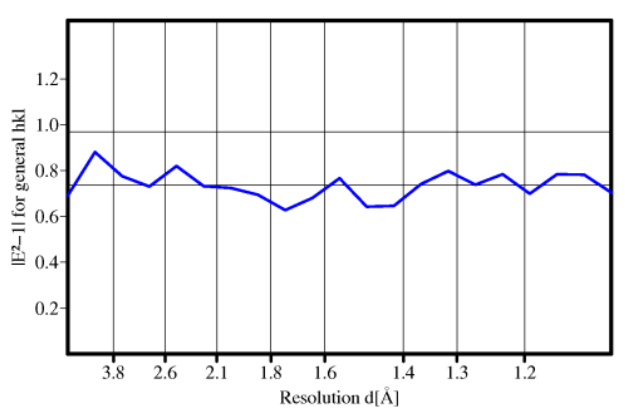

Figure 4.13. $\left|\mathrm{E}^{2}-1\right|$ graph after merging all data in SADABS cell was ruled out on the basis of $\left|E^{2}-1\right|$ values, which were not suspiciously low (Figure 4.13). Other statistical indications were also deemed to be normal, e.g. Rint values for different Laue groups or the $F_{\text {calc }}$ vs. $F_{\text {obs }}$ plot during structure refinement. In the end, the structure was solved by dual space recycling and fragment seeding in SHELXD ${ }^{26}$ using the backbone of the structure FALDUS 28 .

Refinement in the lower symmetry space group (C2), with two $\beta$-CD molecules in the asymmetric unit was also tested but did not bring an improvement in the model or $R$-factor and 
the high symmetry space group was therefore favoured. The significantly different crystal packing of form $V$ (see section 4.4.2.) clarifies the failure of the isomorphous replacement.

Due to the high number of parameters and the low number of reflections (Table 4.1), the refinement was subject to cross validation by the $R_{\text {free, }}$ a commonly used cross validation technique in protein crystallography..$^{73} 10 \%$ of the total number of reflections were flagged in XPREP and used subsequently and exclusively to compute the value of $R_{1 \text { free }}$ in $S H E L X L^{74}$. In order to increase the robustness of the refinement, stereochemical restrains generated with the GRADE webserver ${ }^{30}$ were applied to the host and guest molecules. Structure solution gave an incomplete model of the $\beta$-CD molecule, which was subsequently completed throughout a few least squares' cycles of refinement and inspection of Fourier maps. The electron density map of the guest molecule was not clear during the early stage of the refinement but improved after careful location and treatment of several solvent molecules, and suggested an occupancy factor significantly less than 1 ; the nature of the guest molecule was confirmed using Raman spectroscopy.

\subsubsection{Validation of the guest molecule by Raman spectroscopy}

Raman spectroscopy permits a very rapid and qualitative analysis of materials, and is very sensitive for components which are present at low concentration. A series of Raman spectra were collected to probe the presence of the guest molecule, and to evaluate approximately the amount of guest on the basis of the relative intensity between the guest and $\beta$-CD Raman bands. In order to locate the specific bands of $\beta$-CD and paracetamol molecules, spectra were collected at ambient conditions on crystals of $\beta$-CD hydrate, ${ }^{75}$ paracetamol form $\mathrm{I}^{76} \beta$-CD.paracetamol form $\mathrm{I}^{16}$ in addition to the large crystal of form $\mathrm{V}$ at $0.8 \mathrm{GPa}$, three further crystals visible in the DAC were also investigated by Raman spectroscopy (Figure 4.5). All Raman spectra are presented in Figure 4.14 .

The Raman spectra of both $\beta$-CD hydrate and paracetamol form I are complex due to the chemical diversity and different bond types in both compounds. However, singular bands specific to paracetamol molecules can be observed in the $1500-1700 \mathrm{~cm}^{-1}$ range, the first four of which are assigned to an in plane deformation of the phenyl ring and the last one, at $c a$. $1648 \mathrm{~cm}^{-1}$, to the stretching of the carbonyl bond (Figure 4.14). ${ }^{77}$ In the same wavenumber region, no Raman bands are observed in the spectrum of $\beta$-CD hydrate. In the Raman spectrum of $\beta$-CD-paracetamol form I, the bands of paracetamol are preserved. The Raman spectrum of $\beta$-CD.paracetamol form V (crystal 1) shows the same peaks, although much weaker in intensity, confirming the presence of paracetamol in the crystal structure of form V. The high frequency region confirms the preservation of the aromatic $\mathrm{C}-\mathrm{H}$ stretching bands attributable to paracetamol at 3066 and $3013 \mathrm{~cm}^{-}$ ${ }^{1}$ in both forms I and $\mathrm{V}$ of the inclusion complex. A comparison of the relative intensity of $\beta-\mathrm{CD}$ and 
paracetamol bands clearly indicates that the paracetamol signal is substantially lower in form $\mathrm{V}$ than in form I. While effects such as structural changes and orientation of the single crystals examined might cause differences in the Raman bands between two forms, the intensity change is considerable and can only be explained by the release of paracetamol molecules from inside $\beta$-CD cavities.

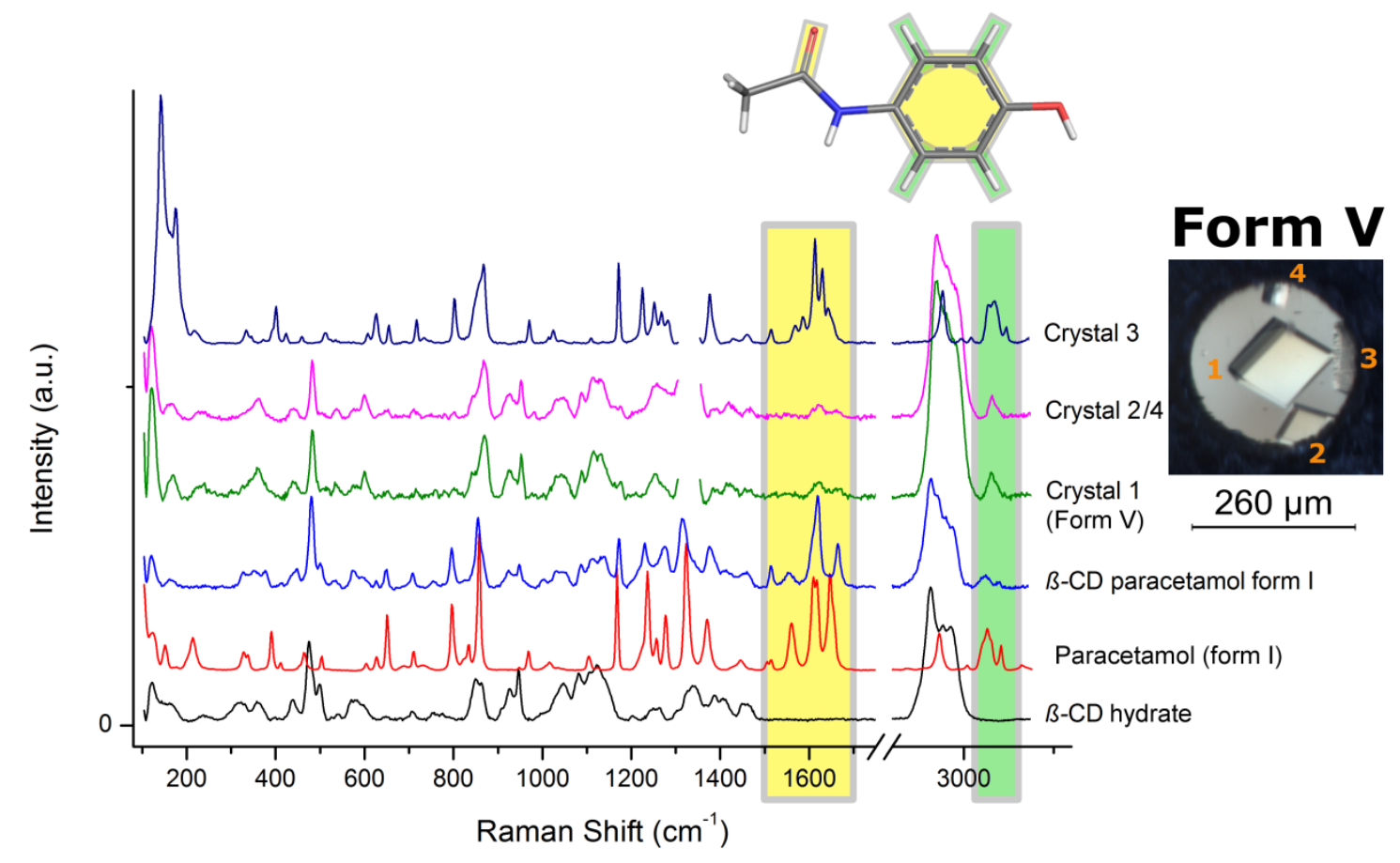

Figure 4.14. Raman spectra of $\beta$-CD hydrate, paracetamol form I, $\beta$-CD'paracetamol form I and the three crystals obtained at high-pressure in a DAC (see Figure 4.5). Characteristic paracetamol bands are highlighted. The Raman spectrum of the small crystal 4 is almost identical to that of crystal 2, hence only one spectrum is shown.

To confirm the theory of partial decomplexation observed for form $\mathrm{V}$ at high-pressure, and to locate the "missing" paracetamol, Raman spectra on crystals 2, 3 and 4 contained in the DAC as well as the liquid part were collected. Raman spectrum of the latter presented very strong fluorescence and Raman bands were undistinguishable (Figure 4.18). The composition of the solution phase therefore remains unknown. Whilst the Raman spectrum of crystal 2 is almost identical to that of $\beta$-CD.paracetamol form $\mathrm{V}$ (crystal 1), crystal 3 gave a Raman spectrum highly similar to that of paracetamol with some differences in peak intensities. Subsequent X-ray diffraction on crystal 3 using synchrotron radiation (microfocus beam on station P02.2 at PETRA) confirmed that crystal 3 is the orthorhombic form of paracetamol. This is a likely confirmation that the stoichiometric (1:1) crystal of $\beta$-CD.paracetamol form I undergoes dissolution upon pressure increase in the DAC, and recrystallises at higher pressures to give a partial inclusion complex form $\mathrm{V}$, as well as paracetamol. 


\subsubsection{Finalising the refinement}

Once the existence of the guest molecule in the crystal of $\beta$-CD.paracetamol form $V$ was confirmed by means of Raman spectroscopy, modelling of paracetamol inside $\beta$-CD cavity proceeded in a fairly straightforward manner: after attributing $c a .50 \%$ of the electron density to water molecules inside $\beta$-CD cavity, the difference electron density maps of the phenyl ring as well as part of the acetamide moiety were visible (Figure 4.15.a). Because of the low site occupancy of paracetamol, parts of the electron density maps can also be modelled with water molecules (Figure 4.15.c). For modelling paracetamol molecule, interatomic distances and angles were subject to restraints using target values generated using the GRADE Web server..$^{30}$ The site-occupancy factor of the guest molecule was refined to complement the one of water molecules sharing the same position making sure that the sum of both sites did not exceed unity, and it converged to a value of ca. 0.3, which was subsequently constrained during the refinement. The atomic displacement parameters of paracetamol were refined isotropically and constrained to have equal values.

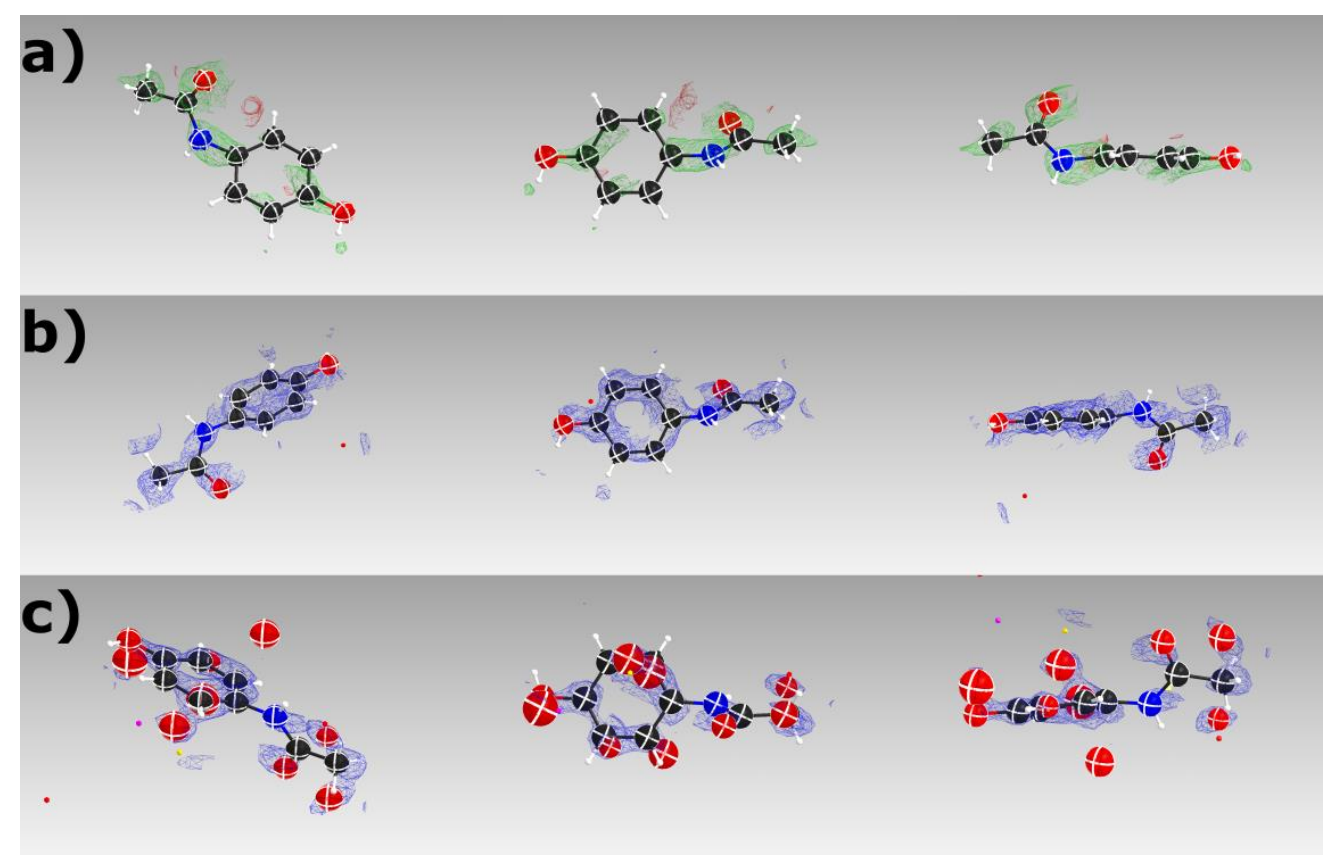

Figure 4.15. Structural model and electron density maps of paracetamol and water molecules generated with shelXle. (a) Paracetamol model fitted in the $F_{\text {obs }}-F_{\text {calc }}$ maps before the refinement (maps at 0.30 e- $/ \AA^{3}$ ); (b) $F_{\text {obs }}$ of paracetamol alone after the refinement (maps at $\left.0.80 \mathrm{e}-/ \AA^{3}\right) ;(\mathrm{c}) F_{\text {obs }}$ of paracetamol and water molecules sharing the same sites (maps at $0.80 \mathrm{e}-/ \AA^{3}$ ).

Although it is clear that form $\mathrm{V}$ contains a very large amount of water, some uncertainty is associated with reporting an accurate value. In order to corroborate the water content in a crystal structure, authors commonly report the use of thermal analysis methods. ${ }^{16,43,59}$ Such analysis requires considerable quantities of sample and are performed at conditions of ambient pressure or modest high pressures. Given form $\mathrm{V}$ is not recoverable to ambient pressure, such analysis could 
not be carried out. Instead, in addition to the Rfree method, calculation of the average non-hydrogen atomic volume was adopted to validate the model..$^{78}$ The average atomic volume per non-hydrogen atom, which is commonly accepted to be $18 \AA^{3}$ for crystals of organic compounds at ambient pressure conditions, was calculated for all $C 2221$ and $P 21$ structures compared in this work. All values are summarised in Table 4.7 and give an average value of $18.6 \AA^{3}$. This value is not very sensitive to changes in temperature but is extremely sensitive to pressure, which allows large variations in unit-cell volumes, as is detailed in Table 4.8 (see Appendix). Overall, the assignment of water molecules was subject to 1) careful inspection of the electron density maps, 2) careful assignment/refinement of the site-occupancy factors and displacement parameters, 3) checking the stability of the model after applying random shifts of $0.4 \AA$ to the coordinates using the SHELXL command WIGL, 4) chemical meaningfulness of the geometries and inspection of intermolecular interactions especially $\mathrm{H}$-bonds network formation.

The structure of $\beta$-CD.paracetamol contains over 24 water molecules per formula unit distributed over 47 positions, of which only 9 are fully ordered and fully occupied outside the cavity. Using this number, the atomic volume per non-hydrogen atom for form $\mathrm{V}$ is $18.75 \AA^{3}$. This value is in our opinion quite large for such a system at $0.8 \mathrm{GPa}$, and the presence of further highly disordered water molecules in regions of low diffuse electron density appears likely.

\subsubsection{Compression study of form I:}

In order to study a possible pressure-induced transition of form I to the low-temperature form transition which proceeds in a single-crystal-to-single-crystal manner at $120 \mathrm{~K},{ }^{17}$ compression experiments on form I were carried out in suitable pressure-transmitting media. Water cannot be used here as dissolution takes place when pressure is applied to the system. To overcome this problem, we switched to 1) a more hydrophobic and bulky perfluorinated oil (FC43), and 2) tetrahydrofuran (THF), in which $\beta$-CD is poorly soluble. ${ }^{79}$

The structure of form I was used as a starting model for refinement of the compressed structures. Redetermination of the crystal structure of form I was carried out in house. To overcome the usual problems associated with the low completeness of high-pressure data, the number of restraints on both the host and guest molecules were maximised using the GRADE web server, and $10 \%$ of each data set was flagged to compute $R_{1 \text { free. The WIGL command was }}$ introduced systematically at different stages of the refinement, and electron density maps were carefully inspected to investigate any structural changes associated with each of the host, guest and solvent molecules. Crystallographic data are summarised in Table 4.4. 
Table 4.4. Crystallographic data of the compression study of $\beta$-CD·paracetamol form I inclusion complex

\begin{tabular}{|c|c|c|c|c|c|}
\hline & Form Ia & $\begin{array}{c}\text { FC43 } \\
0.13 \mathrm{GPa}\end{array}$ & $\begin{array}{c}\mathrm{FC} 43 \\
0.95 \mathrm{GPa}\end{array}$ & $\begin{array}{c}\text { THF } \\
0.16 \mathrm{GPa}\end{array}$ & $\begin{array}{c}\mathrm{THF} \\
0.60 \mathrm{GPa}\end{array}$ \\
\hline Formula & $\begin{array}{l}\mathrm{C}_{42} \mathrm{H}_{72} \mathrm{O}_{35} \\
\mathrm{C}_{8} \mathrm{H}_{9} \mathrm{NO}_{2} \\
8.6(\mathrm{H} 2 \mathrm{O})\end{array}$ & $\begin{array}{c}\mathrm{C}_{42} \mathrm{H}_{72} \mathrm{O}_{35} \\
\mathrm{C}_{8} \mathrm{H}_{9} \mathrm{NO}_{2}, 8.92\left(\mathrm{H}_{2} \mathrm{O}\right)\end{array}$ & $\begin{array}{c}\mathrm{C}_{42} \mathrm{H}_{72} \mathrm{O}_{35} \\
\mathrm{C}_{8} \mathrm{H}_{9} \mathrm{NO}_{2} \\
10.15\left(\mathrm{H}_{2} \mathrm{O}\right)\end{array}$ & $\begin{array}{l}\mathrm{C}_{42} \mathrm{H}_{72} \mathrm{O}_{35} \\
\mathrm{C}_{8} \mathrm{H}_{9} \mathrm{NO}_{2} \\
5.83\left(\mathrm{H}_{2} \mathrm{O}\right)\end{array}$ & $\begin{array}{c}\mathrm{C}_{42} \mathrm{H}_{72} \mathrm{O}_{35} \\
0.5\left(\mathrm{C}_{8} \mathrm{H}_{9} \mathrm{NO}_{2}\right) \\
8.45\left(\mathrm{H}_{2} \mathrm{O}\right)\end{array}$ \\
\hline Space group & $\mathrm{C} 2$ & C2 & $\mathrm{C} 2$ & $\mathrm{C} 2$ & $C 2$ \\
\hline$a / \AA$ & $18.9343(8)$ & $18.832(3)$ & $18.497(12)$ & 18.8131(16) & 18.6154(16) \\
\hline$b / \AA ̊$ & $24.4136(11)$ & $24.363(3)$ & $24.051(14)$ & $24.321(2)$ & $24.2776(8)$ \\
\hline$c / \AA ̊$ & $15.5941(7)$ & $15.516(2)$ & $15.189(9)$ & $15.5384(9)$ & $15.340(3)$ \\
\hline$\alpha /{ }^{\circ}$ & 90 & 90 & 90 & 90 & 90 \\
\hline$\beta /^{\circ}$ & $110.798(3)$ & $110.713(5)$ & $110.272(17)$ & $111.088(8)$ & $110.302(16)$ \\
\hline$\gamma /{ }^{\circ}$ & 90 & 90 & 90 & 90 & 90 \\
\hline$V / \AA^{3}$ & $6738.7(5)$ & $6658.4(16)$ & $6339(7)$ & $6633.5(10)$ & $6501.9(15)$ \\
\hline$Z^{\prime}$ & 1 & 1 & 1 & 1 & 1 \\
\hline$D_{\text {calc }} / \mathrm{g} \mathrm{cm}^{-3}$ & 1.403 & 1.424 & 1.516 & 1.380 & 1.373 \\
\hline Pressure $^{b}$ & $0.1 \mathrm{MPa}$ & $0.13 \mathrm{GPa}$ & $0.95 \mathrm{GPa}$ & $0.16 \mathrm{GPa}$ & $0.60 \mathrm{GPa}$ \\
\hline$T / K$ & $298(2)$ & $298(2)$ & $298(2)$ & $298(2)$ & $298(2)$ \\
\hline $\begin{array}{c}\text { Meas./uniq./obs. } \\
\text { Reflections }^{c}\end{array}$ & $\begin{array}{c}15790 / 5481 / \\
4004\end{array}$ & $\begin{array}{c}13979 / 3736 / \\
3324\end{array}$ & $\begin{array}{c}7161 / 1993 / \\
1636\end{array}$ & $\begin{array}{c}12792 / 5335 / \\
3932\end{array}$ & $\begin{array}{c}6985 / 2442 / \\
2048\end{array}$ \\
\hline Parameters & 847 & 842 & 473 & 835 & 409 \\
\hline Restraints & 2365 & 2337 & 1315 & 2321 & 1231 \\
\hline$R_{\text {int }}$ & 0.0705 & 0.0493 & 0.1123 & 0.0850 & 0.0622 \\
\hline$d_{\text {max }} / \AA ̊$ & 1.0 & 1.10 & 1.09 & 0.90 & 1.10 \\
\hline $\begin{array}{l}\text { Completeness to } \\
\qquad d_{\max }\end{array}$ & 0.896 & 0.778 & 0.794 & 0.663 & 0.526 \\
\hline$R_{1}^{e}$ & 0.0750 & 0.0721 & 0.14 & 0.941 & 0.0994 \\
\hline $\begin{array}{c}\text { Rfree }_{\text {e }} \\
\text { (obs. Reflections) } f\end{array}$ & $\begin{array}{c}0.1012 \\
(605)\end{array}$ & $\begin{array}{c}0.1066 \\
(383)\end{array}$ & $\begin{array}{c}0.1803 \\
(174)\end{array}$ & $\begin{array}{c}0.1225 \\
(431)\end{array}$ & $\begin{array}{c}0.1202 \\
(223)\end{array}$ \\
\hline$w R_{2} g$ & 0.1852 & 0.1852 & 0.3243 & 0.2469 & 0.2562 \\
\hline
\end{tabular}

a Redetermination in this work. ${ }^{b}$ These values refer to the pressure at which diffraction data were collected. c Criteria for observed reflections: $I>2 \sigma(I)$. ${ }^{\mathrm{d}}$ Criteria for observed reflections: $I>3 \sigma(I) .{ }^{\mathrm{e}}\left[F^{2}>2 \sigma\left(F^{2}\right)\right] .{ }^{\mathrm{f}} 10 \%$ of unique reflections $\mathrm{g}\left(F^{2}\right)$.

\subsubsection{Compression study in FC43}

The principal structural difference between $\beta$-CD.paracetamol form I at ambient conditions and at $0.13 \mathrm{GPa}$ is the ordering of one of the primary hydroxy groups: at ambient-pressure conditions all hydroxy groups exhibit the preferred (-)-gauche conformation with three of them further disordered to the non-favoured (+)-gauche conformation. Of these three gauche conformers, one contributes to the stability of a displaced tail-to-tail dimer, a second is H-bonded to the hydroxy group of the guest molecule, and the third one has both types of contacts. The (-)-gauche conformer of the latter, originally $\mathrm{H}$-bonded to a water molecule at ambient-pressure conditions, rotates to the (+)-gauche conformer at $0.13 \mathrm{GPa}$. In addition to this conformational change, a previously unobserved $\mathrm{H}_{2} \mathrm{O}$ molecule with site-occupancy factor of $1 / 4$ appears inside the $\beta$-CD cavity creating a H-bond with the hydroxy group of paracetamol. The two positions of the guest molecule are comparable (50:50 disorder) and do not change significantly (Figure 4.16.a-b) between the two pressure points. At $0.9 \mathrm{GPa}$ (Figure 4.16.c), the phenyl ring of one of the guest molecules rotates to an almost orthogonal position compared to that of the phenyl ring of the second paracetamol molecule: the dihedral angle between the phenyl rings of the two positions, 
calculated by means of the corresponding least-square planes containing all phenyl-C atoms, are 44,50 and $81^{\circ}$ for ambient pressure, 0.13 and 0.95 GPa structures, respectively. The rotation of the ring observed at $0.95 \mathrm{GPa}$ could be related in fact to water ordering inside the channels, which is also observed at that pressure. Having gone beyond the hydrostatic limit of the fluid (0.9 GPa observed in this work), no further data points were collected.
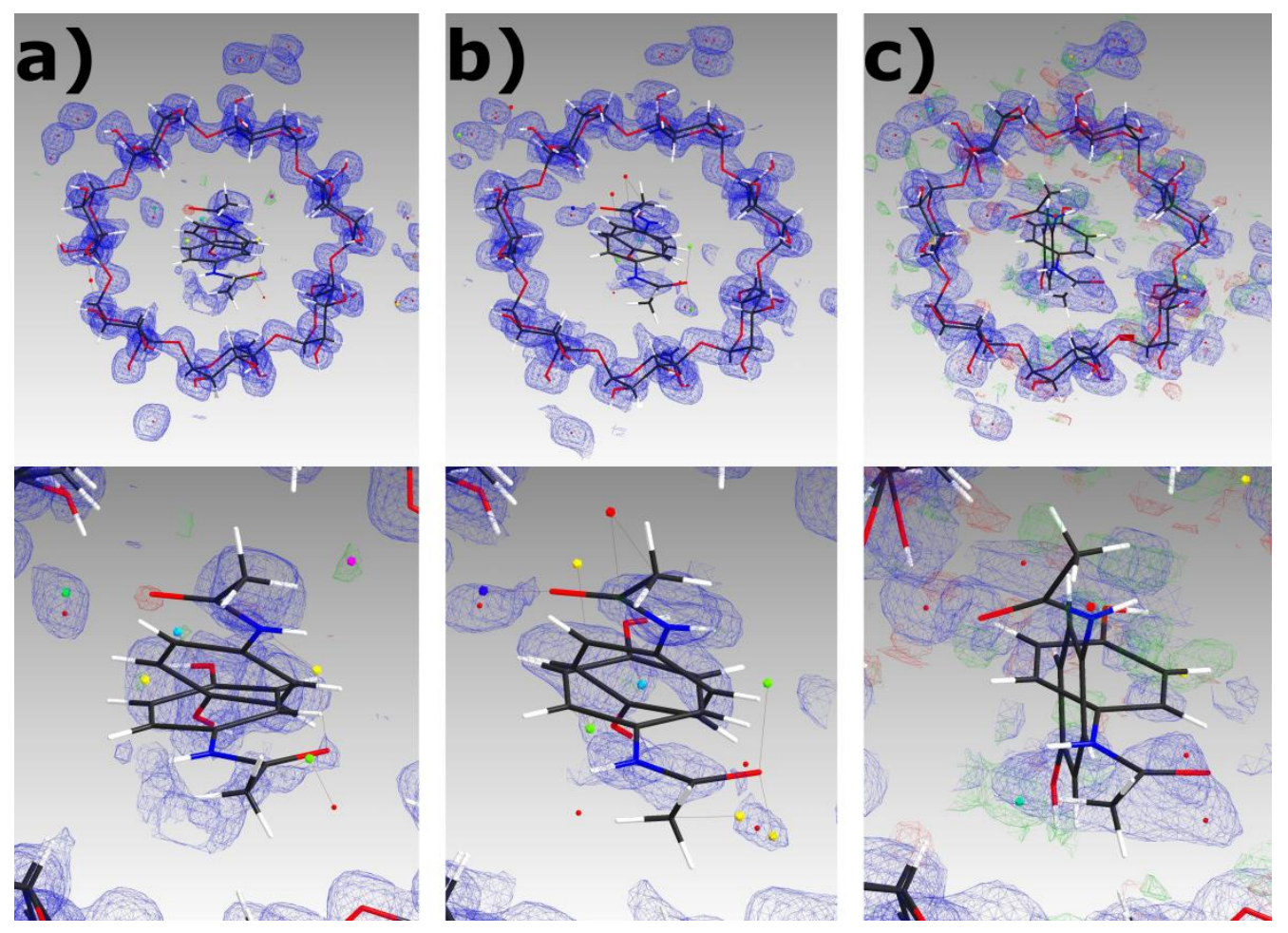

Figure 4.16. Compression study of $\beta$-CD·paracetamol form I in FC43. (a) structure of the complex at room temperature; (b) the complex at $0.13 \mathrm{GPa}$; (c) the complex at $0.95 \mathrm{GPa}$. The apparent change in the paracetamol hydroxyl $\mathrm{H}$-atom at high pressure was not determined experimentally but arises from the riding restraint command chosen for refinement (AFIX 83 command in SHELXL), i.e. it may be an artefact.

\subsubsection{Compression study in THF}

Similarly to what was observed in FC43, compression of $\beta$-CD·paracetamol form I in THF generates ordering of the same primary hydroxy group to a (+)-gauche conformer. However in $\mathrm{THF}$, we observed an overall deficiency in the electron density maps inside the cavity interpreted as a loss of water content (Figure 4.17). At $0.6 \mathrm{GPa}$ the loss of electron density is very marked to the extent that half of the guest's contribution is lost: the paracetamol molecule can only be refined as being ordered and showing large isotropic displacement parameters and an overall site occupancy factor of 0.5 (Figure 4.17.c). In addition, the structure at 0.6 GPa partially recovers the water loss observed at $0.16 \mathrm{GPa}$, taking up 2.4 water molecules inside the cavity.

The decrease in water content at $0.16 \mathrm{GPa}$ can be related to the crystal environment: THF is water miscible, and the channel structure of $\beta$-CD.paracetamol form I favours the mobility of the 
solvents molecules, thus water molecules might find their way out of the crystal, accounting for the loss of electron density. At $0.6 \mathrm{GPa}$, the loss of guest molecules must be related to a partial decomplexation process: THF is known to be a good solvent for paracetamol at ambient pressure conditions, $^{80}$ and it is not unlikely that THF favours to some extent a gradual dissolution of paracetamol molecules and their substitution by water molecules. As far as the data can tell, there is no evidence for inclusion of THF in the structure of the complex. Further pressure increase to 1 GPa exacerbates loss of data quality: reflections found to be severely split and resolution dropped below $1.4 \AA$; however, data indexing still indicates the unit-cell parameters of form I. Figure 4.6.e shows the resulting crystal form I in THF at $1 \mathrm{GPa}$. Poor data quality could be associated with an advanced stage of decomplexation associated with visible deterioration of the crystal.
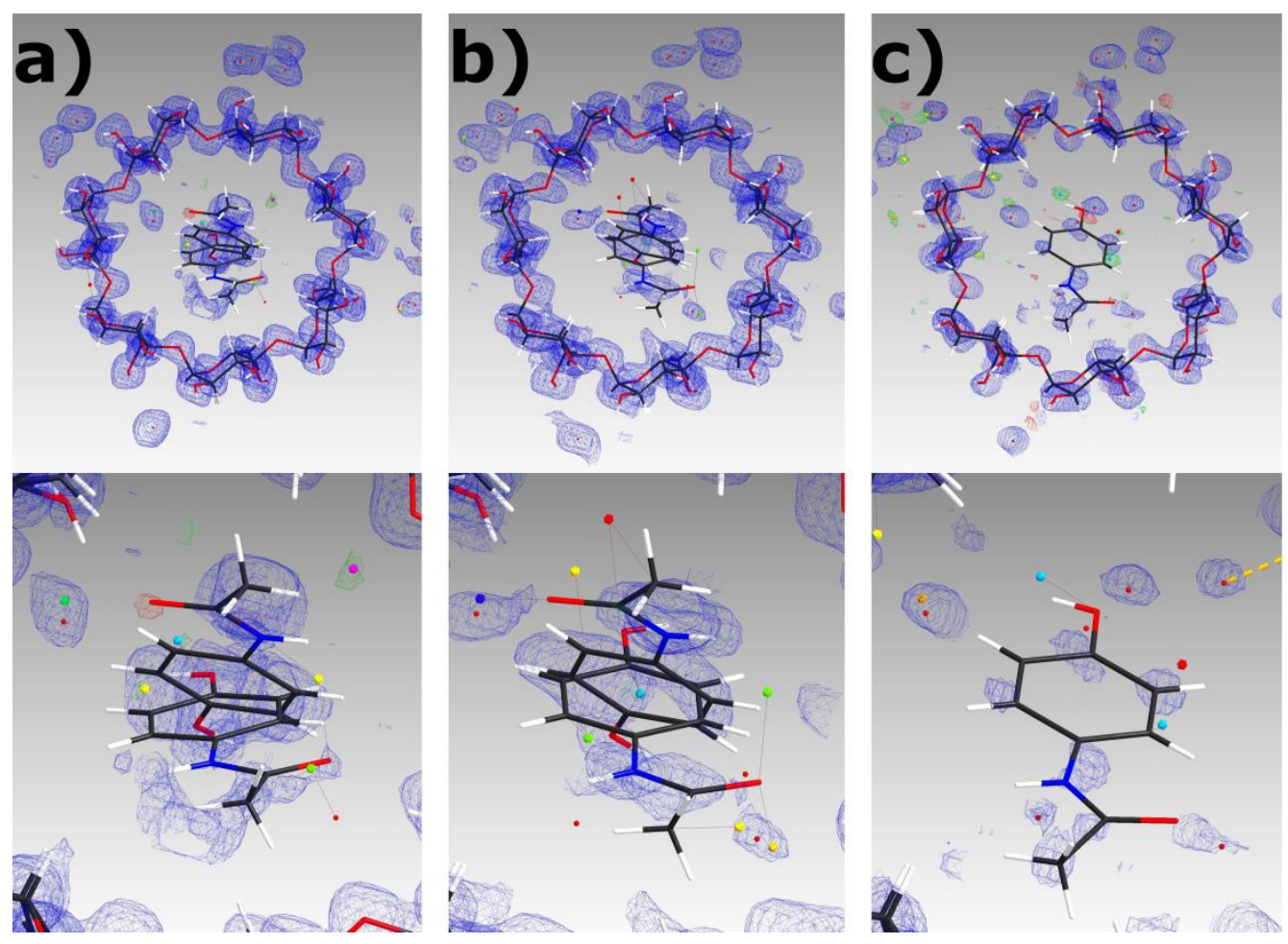

Figure 4.17. Compression study of $\beta$-CD·paracetamol form I in THF. (a) structure of the complex at room temperature; (b) the complex at $0.16 \mathrm{GPa}$; (c) the complex at $0.60 \mathrm{GPa}$. The apparent change in the paracetamol hydroxyl H-atom at high pressure was not determined experimentally but arises from the riding restraint command chosen for refinement (AFIX 83 command in SHELXL), i.e. it may be an artefact. 


\subsection{Conclusion}

A new crystal form of $\beta$-CD.paracetamol inclusion complex, form $V$, has been characterised by means of single-crystal X-ray diffraction using synchrotron radiation and Raman spectroscopy. Form V crystallises exclusively at high pressure and, unlike similar inclusion complexes, it incorporates a very large number of water molecules. Similar dimeric inclusion complexes crystallising in the C2221 space group pack in parallel and displaced dimeric arrangements related by a 21 screw axis, known as a chessboard packing motif: form $\mathrm{V}$ exhibits the same packing mode with compressed screw channels and a larger unit cell volume to incorporate the excess of water molecules. The packing mode of form $\mathrm{V}$ has also been compared to that of $P 2{ }_{1} \beta$-CD inclusion complexes crystallising in the screw channel packing type. The Crystal Packing Similarities plugin available in MERCURY has been used to output RMSD differences between C2221 and $P 21$ structures, which were ascribed to the spacing and arrangement of the screw channels towards the equivalent orthorhombic $a c$ and the monoclinic (10-1) planes. The arrangement of the screw channels in $P 21$ structures is almost perpendicular to the one in $C 2221$ structures. The analysis shows also that form $\mathrm{V}$ structure of the title compound is an intermediate structure between both $C 222_{1}$ and $P 21$ groups.

When compressed in water, form I dissolves as function of increasing pressure and recrystallises at higher pressures into form $\mathrm{V}$. The dissolution process is an essential step to incorporate more water molecules in the crystal structure as expressed by the $274 \AA^{3}$ increase per $\mathrm{CD}$ unit, while the dimeric arrangement of $\beta$ - $\mathrm{CD}$ molecules is conserved in both forms confirming the robustness of this motif.

Compression studies of form I using FC43 and THF as pressure transmitting media show that in the explored pressure ranges 1) single-crystal-to-single-crystal phase transitions do not occur in both systems; 2) water ordering is associated with the nature of the pressure transmitting medium as well as pressure: ordering is obtained when using FC43 while THF "draws out" water from the structure; and 3) the guest molecule can rotate as well as leave the $\beta$-CD cavity.

This work exemplifies the great structural variability of $\beta$-CD.paracetamol inclusion complexes and proves the virtues of the use of high-pressure techniques to explore the solid-state behaviour of CD-based inclusion complexes. 


\subsection{References}

(1) French, D.; Levine, M. L.; Pazur, J. H.; Norberg, E. Studies on the Schardinger Dextrins; the Preparation and Solubility Characteristics of Alpha, Beta and Gamma Dextrins. J. Am. Chem. Soc. 1949, 71 (1), 353-356.

(2) Irie, T.; Uekama, K. Pharmaceutical Applications of Cyclodextrins. III. Toxicological Issues and Safety Evaluation. J. Pharm. Sci. 1997, 86 (2), 147-162.

(3) Harata, K. Structural Aspects of Stereodifferentiation in the Solid State. Chem. Rev. 1998, 98 (5), 1803-1827.

(4) Uekama, K.; Hirayama, F.; Irie, T. Cyclodextrin Drug Carrier Systems. Chem. Rev. 1998, 98 (5), 2045-2076.

(5) Martin Del Valle, E. M. Cyclodextrins and Their Uses: A Review. Process Biochem. 2003, 39 (9), 1033-1046.

(6) Davis, M. E.; Brewster, M. E. Cyclodextrin-Based Pharmaceutics: Past, Present and Future. Nat. Rev. Drug Discov. 2004, 3 (12), 1023-1035.

(7) Loftsson, T.; Jarho, P.; Másson, M.; Järvinen, T. Cyclodextrins in Drug Delivery. Expert Opin. Drug Deliv. 2005, 2 (c), 335-351.

(8) Rasheed, A.; Kumar, A. C. K.; Sravanthi, V. V. N. S. S. Cyclodextrins as Drug Carrier Molecule: A Review. Sci. Pharm. 2008, 76 (4), 567-598.

(9) Ceborska, M. Interactions of Native Cyclodextrins with Biorelevant Molecules in the Solid State : A Review. Curr. Org. Chem. 2014, 18, 1878-1885.

(10) Rekharsky, M. V.; Inoue, Y. Complexation Thermodynamics of Cyclodextrins. Chem. Rev. 1998, 98, 1875-1917.

(11) Chen, W.; Chang, C.-E.; Gilson, M. K. Calculation of Cyclodextrin Binding Affinities: Energy, Entropy, and Implications for Drug Design. Biophys. J. 2004, 87 (5), 3035-3049.

(12) Biwer, A.; Antranikian, G.; Heinzle, E. Enzymatic Production of Cyclodextrins. Appl. Microbiol. Biotechnol. 2002, 59, 609-617.

(13) Szejtli, J. Introduction and General Overview of Cyclodextrin Chemistry. Chem. Rev. 1998, 98, 1743-1753.

(14) Szente, L.; Szemán, J. Cyclodextrins in Analytical Chemistry: Host-Guest Type Molecular Recognition. Anal. Chem. 2013, 85 (17), 8024-8030.

(15) Ellis, F. Paracetamol - a Curriculum Resource; Osborne, C., Pack, M., Eds.; Royal Society of Chemistry: London, United Kingdom, 2002.

(16) Caira, M. R.; Dodds, D. R. Inclusion of Nonopiate Analgesic Drugs in Cyclodextrins . II . XRay Structure of a 1:1 $\beta$-Cyclodextrin - Acetaminophen Complex. J. Incl. Phenom. Macrocycl. Chem. 2000, 38, 75-84.

(17) Fabbiani, F. P. A.; Buth, G.; García, R. G.; Lahoz, F. J.; Paulmann, C.; Saouane, S. Host-Guest Interactions in Cyclodextrin Inclusion Complexes at Extreme Conditions. Acta Crystallogr. Sect. A 2012, A68, S115.

(18) Saouane, S.; Norman, S. E.; Hardacre, C.; Fabbiani, F. P. A. Pinning down the Solid-State Polymorphism of the Ionic Liquid [bmim][PF6]. Chem. Sci. 2013, 4 (3), 1270-1280.

(19) Paulmann, C. CMH and M2B, The University of Hamburg, Hamburg, Germany. CMH and M2B, The University of Hamburg, Hamburg, Germany 2005.

(20) Bruker-AXS. APEX II, Version v2014.1-1; Bruker AXS. APEX II, version v2014.1-1: Madison, Wisconsin, USA 2014. 
(21) Rau, A. W.; Bakueva, L.; Rowlands, J. A. The X-Ray Time of Flight Method for Investigation of Ghosting in Amorphous Selenium-Based Flat Panel Medical X-Ray Imagers. Med. Phys. 2005, 32 (10), 3160-3177.

(22) Mail, N.; O'Brien, P.; Pang, G. Lag Correction Model and Ghosting Analysis for an Indirect Conversion Flat Panel Imager. J. Appl. Clin. Med. Phys. 2007, 8 (3), 137-146.

(23) Bruker-AXS. SAINT Version 8.34A, Madison, Wisconsin, USA. SAINT version 8.34A: Madison, Wisconsin, USA 2013.

(24) Parsons, S. SHADE, 2004, The University of Edinburgh: Scotland. SHADE, 2004, The University of Edinburgh: Edinburgh, Scotland.

(25) Sheldrick, G. M. SADABS Version 2008-1, Bruker-AXS, Madison, Wisconsin, USA. SADABS Version 2008-1, 2008, Bruker-AXS: Madison, Wisconsin, USA 2008.

(26) Sheldrick, G. M. A Short History of SHELX. Acta Crystallogr. Sect. A Found. Crystallogr. 2008, 64, 112-122.

(27) Allen, F. H. The Cambridge Structural Database: A Quarter of a Million Crystal Structures and Rising. Acta Crystallogr. Sect. B Struct. Sci. 2002, 58, 380-388.

(28) Damodharan, L.; Pattabhi, V.; Nagarajan, K. Solvent Interactions with $\beta$-Cyclodextrin as Observed in Crystal Structures. Mol. Cryst. Liq. Cryst. 2004, 423 (1), 17-35.

(29) Hübschle, C. B.; Sheldrick, G. M.; Dittrich, B. ShelXle: A Qt Graphical User Interface for SHELXL. J. Appl. Crystallogr. 2011, 44, 1281-1284.

(30) Grade Web Server, Global Phasing Ltd. http://grade.globalphasing.org/ (GRADE version 1.2.7).

(31) Thorn, A.; Dittrich, B.; Sheldrick, G. M. Enhanced Rigid-Bond Restraints. Acta Crystallogr. Sect. A Found. Crystallogr. 2012, 68, 448-451.

(32) Bonnet, P.; Jaime, C.; Morin-Allory, L. Structure and Thermodynamics of Alpha-, Beta-, and Gamma-Cyclodextrin Dimers. Molecular Dynamics Studies of the Solvent Effect and Free Binding Energies. J. Org. Chem. 2002, 67, 8602-8609.

(33) Saenger, W.; Steiner, T. Cyclodextrin Inclusion Complexes: Host-Guest Interactions and Hydrogen-Bonding Networks. Acta Crystallogr. Sect. A Found. Crystallogr. 1998, 54 (6), 798 805.

(34) Giastas, P.; Yannakopoulou, K.; Mavridis, I. M. Molecular Structures of the Inclusion Complexes $\beta$-cyclodextrin-1,2-bis(4-Aminophenyl)ethane and $\beta$-cyclodextrin-4,4'Diaminobiphenyl; Packing of Dimeric $\beta$-Cyclodextrin Inclusion Complexes. Acta Crystallogr. Sect. B Struct. Sci. 2003, 59 (2), 287-299.

(35) Mentzafos, D.; Mavridis, I. M.; Le Bas, G.; Tsoucaris, G. Structure of the 4-Tert-Butylbenzyl Alcohol- $\beta$-Cyclodextrin Complex. Common Features in the Geometry of $\beta$-Cyclodextrin Dimeric Complexes. Acta Crystallogr. Sect. B Struct. Sci. 1991, 47 (5), 746-757.

(36) Zhao, Y.-L.; Benítez, D.; Yoon, I.; Stoddart, J. F. Inclusion Behavior of Beta-Cyclodextrin with Bipyridine Molecules: Factors Governing Host-Guest Inclusion Geometries. Chem. - An Asian J. 2009, 4 (3), 446-456.

(37) Bruno, I. J.; Cole, J. C.; Edgington, P. R.; Kessler, M.; Macrae, C. F.; McCabe, P.; Pearson, J.; Taylor, R. New Software for Searching the Cambridge Structural Database and Visualizing Crystal Structures. Acta Crystallogr. 2002, B58, 389-397.

(38) Sánchez-Ruiz, X.; Alvarez-Larena, A.; Jaime, C.; Piniella, J. F.; Redondo, J.; Virgili, A.; Sanchez-Ferrando, F.; Germain, G.; Baert, F. Molecular and Crystal Structure of the 1:1 Complex of Adamantanone with $\beta$-Cyclodextrin. Supramol. Chem. 1999, 10 (3), 219-223. 
(39) Macrae, C. F.; Bruno, I. J.; Chisholm, J. a.; Edgington, P. R.; McCabe, P.; Pidcock, E.; Rodriguez-Monge, L.; Taylor, R.; Van De Streek, J.; Wood, P. a. Mercury CSD 2.0 - New Features for the Visualization and Investigation of Crystal Structures. J. Appl. Crystallogr. 2008, 41 (2), 466-470.

(40) Tokuoka, R.; Fujiwara, T.; Tomita, K. I. Structure of the $\beta$-Cyclodextrin $(\beta-C D)$ Inclusion Complex with P-Ethylaniline (PEA). Acta Crystallogr. Sect. B Struct. Crystallogr. Cryst. Chem. 1981, 37 (5), 1158-1160.

(41) Bruno, I. J.; Cole, J. C.; Kessler, M.; Luo, J.; Momerwell, W. D. S.; Purkis, L. H.; Smith, B. R.; Taylor, R.; Cooper, R. I.; Harris, S. E.; et al. Retrieval of Crystallographically-Derived Molecular Geometry Information. J. Chem. Inf. Comput. Sci. 2004, 44 (6), 2133-2144.

(42) Liu, Y.; Zhao, Y. L.; Zhang, H. Y.; Li, X. Y.; Liang, P.; Zhang, X. Z.; Xu, J. J. Supramolecular Polypseudorotaxane with Conjugated Polyazomethine Prepared Directly from Two Inclusion Complexes of ??-Cyclodextrin with Tolidine and Phthaldehyde. Macromolecules 2004, 37 (17), 6362-6369.

(43) Caira, M. R.; De Vries, E.; Nassimbeni, L. R.; Jacewicz, V. W. Inclusion of the Antidepressant Paroxetine in Beta-Cyclodextrin. J. Incl. Phenom. 2003, 46, 37-42.

(44) Le Bas, G.; Rango, C. de; Rysanek, N.; Tsoucaris, G.; de Rango, C.; Rysanek, N.; Tsoucaris, G. Chiral Conformations Induced by Cyclodextrin. J. Incl. Phenom. 1984, 2 (3-4), 861-867.

(45) Enright, G. D.; Udachin, K. a.; Ripmeester, J. a. Complex Packing Motifs Templated by a Pseudo-Spherical Guest: The Structure of $\beta$-Cyclodextrin-adamantane Inclusion Compounds. CrystEngComm 2010, 12 (5), 1450.

(46) Hamilton, J. A. Structure of Inclusion Complexes of Cyclomaltoheptaose (Cycloheptaamylose): Crystal Structure of the 1-Adamantanemethanol Adduct. Carbonhydrate Res. 1985, 142, 21-37.

(47) Alston, D. R.; Slawin, A. M. Z.; Stoddart, J. F.; Williams, D. J.; Zarzycki, R. Second Sphere Coordination Adducts of Phosphane-Transition Metal Complexes with $\beta$-Cyclodextrin and Its Methylated Derivative. Angew. Chem. Int. Ed. Engl. 1988, 27 (9), 1184-1185.

(48) Ceborska, M.; Asztemborska, M.; Luboradzki, R.; Lipkowski, J. Interactions with BetaCyclodextrin as a Way for Encapsulation and Separation of Camphene and Fenchene. Carbohydr. Polym. 2013, 91 (1), 110-114.

(49) Bobek, M. M.; Giester, G.; Ka, H. A "Sugar-Coated" Carbene Precursor : A Single Crystal XRay Diffraction and NMR Study. Tetrahedron Lett. 2000, 41, 5663-5667.

(50) Dean, P. M. Structural and Thermal Characterization of Cyclodextrin-Suprofen Complexes. Aust. J. Chem. 2007, 60, 133-138.

(51) Stellenbooma, N.; Huntera, R.; Cairaa, M. R.; Bournea, S. A.; Barbieria, M. Inclusion of the Allicin Mimic S-P-Tolyl T-Butylthiosulphinate in $\beta$-Cyclodextrin. Supramol. Chem. 2009, 21, 611-617.

(52) Nakanishi, I.; Fujiwara, T.; Tomita, K. Structure of Beta-Cyclodextrin-Phenobarbital Complex. Acta Crystallogr. 1984, A40, C78C.

(53) Hamilton, J. A.; Chen, L. Crystal Structure of B-Cyclodextrin RS(+-)-2-(3-Phenoxyphenyl)Propionic Acid Hydrate. ACA Abstr. Pap. 1986, 14, 50.

(54) Hamilton, J. A.; Chen, L. Crystal Structures of an Inclusion Complexes of Beta-Cyclodextrin with Racemic Fenoprofen: Direct Evidence for Chiral Recognition. J. Am. Chem. Soc. 1988, 110 (17), 5833-5841.

(55) Liu, Y.; Zhao, Y.; Chen, Y.; Guo, D. Assembly Behavior of Inclusion Complexes of $\beta$ Cyclodextrin with 4-Hydroxyazobenzene and 4-Aminoazobenzene. Org. Biomol. Chem. 2005, 


\section{3, 584-591.}

(56) Hamilton, J. A.; Chen, L. Crystal Structures of Inclusion Complexes of Beta-Cyclodextrin with S-(+)- and (R)-(-)-Fenoprofen. J. Am. Chem. Soc. 1988, 110 (13), 4379-4391.

Takashima, Y.; Oizumi, Y.; Sakamoto, K.; Miyauchi, M.; Kamitori, S.; Harada, A. Crystal Structure of the Complex of $\beta$-Cyclodextrin with Bithiophene and Their Oxidative Polymerization in Water. Macromolecules 2004, 37 (11), 3962-3964.

(58) Takashima, Y.; Sakamoto, K.; Oizumi, Y.; Yamaguchi, H.; Kamitori, S.; Harada, A. Complex Formation of Cyclodextrins with Various Thiophenes and Their Polymerization in Water: Preparation of Poly-Pseudo-Rotaxanes Containing Poly(thiophene)s. J. Incl. Phenom. Macrocycl. Chem. 2006, 56, 45-53.

(59) Caira, M. R.; Griffith, V. J.; Nassimbeni, L. R.; Oudtshoorn, B. Van. X-Ray Structures of 1:1 Complexes of (L)-Menthol with $\beta$-Cyclodextrin and Permethylated $\beta$-Cyclodextrin. Supramol. Chem. 1996, 7 (2), 119-124.

(60) Gromov, S. P.; Nazarov, V. B.; Avakyan, V. G.; Fomina, M. V.; Vedernikov, A. I.; Kuz'Mina, L. G.; Vershinnikova, T. G.; Lobova, N. a.; Rudyak, V. Y.; Alfimov, M. V.; et al. Photoinduced Protonation and Mechanical Motion in the Cyclodextrin Cavity: Synthesis, Structure and Spectral Properties of 4-(2-Napthyl)pyridine and Their Pseudorotaxane Complexes. J. Photochem. Photobiol. A Chem. 2011, 217 (1), 87-99.

(61) Brett, T. J.; Stezowski, J. J.; Liu, S.; Coppens, P. The $20 \mathrm{~K}$ Structure of P-Amino-P'Nitrobiphenyl in the Non-Constraining Environment of Its $\beta$-Cyclodextrin Inclusion Complex. Chem. Commun. 1999, No. 6, 551-552.

(62) Ceborska, M.; Asztemborska, M.; Lipkowski, J. Rare "head-to-Tail" Arrangement of Guest Molecules in the Inclusion Complexes of (+)- and (-)-Menthol with $\beta$-Cyclodextrin. Chem. Phys. Lett. 2012, 553, 64-67.

(63) Cappadona, T.; Daniels, L.; Siddiquee, T. Host-Guest Complex of $\beta$-Cyclodextrin and Disulfide Form of 4-Aminothiophenol. Appl. Sci. 2012, 2 (4), 773-779.

(64) Nakanishi, I.; Arai, M.; Fujiwara, T.; Tomita, K. X-Ray Structural Studies on Two Forms of ß-Cyclodextrin Barbital Complexes. J. Incl. Phenom. 1984, 2 (3), 689-699.

(65) Fernandes, J. A.; Almeida Paz, F. A.; Braga, S. S.; Ribeiro-Claro, P. J. A.; Rocha, J. Inclusion of Potassium 4,4'-Biphenyldicarboxylate into $\beta$-Cyclodextrin: The Design and Synthesis of an Organic Secondary Building Unit. New J. Chem. 2011, 35 (6), 1280.

(66) Liu, Y.; Zhao, Y.-L.; Zhang, H.-Y.; Yang, E.-C.; Guan, X.-D. Binding Ability and Assembly Behavior of $\beta$-Cyclodextrin Complexes with 2,2'-Dipyridine and 4,4'-Dipyridine. J. Org. Chem. 2004, 69 (10), 3383-3390.

(67) Shen, X. J.; Chen, H. L.; Yu, F.; Zhang, Y. C.; Yang, X. H.; Li, Y. Z. A New Type of [2] and [3]pseudorotaxane Composed of $\beta$-Cyclodextrin and Bisimidazolyl Compounds. Tetrahedron Lett. 2004, 45 (36), 6813-6817.

(68) Aree, T.; Chaichit, N.; Engkakul, C. Polymorphism in $\beta$-Cyclodextrin-benzoic Acid Inclusion Complex: A Kinetically Controlled Crystal Growth according to the Ostwald's Rule. Carbohydr. Res. 2008, 343 (14), 2451-2458.

(69) Liu, Y.; Zhong, R.-Q.; Zhang, H.-Y.; Song, H.-B. A Unique Tetramer of 4:5 $\beta$-Cyclodextrinferrocene in the Solid State. Chem. Commun. (Camb). 2005, No. 17, 2211-2213.

(70) Tsorteki, F.; Bethanis, K.; Pinotsis, N.; Giastas, P.; Mentzafos, D. Inclusion Compounds of Plant Growth Regulators in Cyclodextrins. V. 4-Chlorophenoxyacetic Acid Encapsulated in B-Cyclodextrin and heptakis(2,3,6-Tri-O-Methyl)-B-Cyclodextrin. Acta Crystallogr. 2005, B61, 207-217. 
(71) Hamilton, J. A.; Steinrauf, L. K.; Etten, R. L. Van. Interrelated Space Groups Observed for Complexes of Cycloheptaamylose with Small Organic Molecules. Acta Crystallogr. B. 1968, 24 (11), 1560-1562.

(72) Malpezzi, L.; Fronza, G.; Fuganti, C.; Mele, A.; Brückner, S. Crystal Architecture and Conformational Properties of the Inclusion Complex, Neohesperidin DihydrochalconeCyclomaltoheptaose ( $\beta$-Cyclodextrin), by X-Ray Diffraction. Carbohydr. Res. 2004, 339 (12), 2117-2125.

(73) Brünger, A. T. Free R Value: A Novel Statistical Quantity for Assessing the Accuracy of Crystal Structures. Nature 1992, 355, 472-475.

(74) Sheldrick, G. M. Crystal Structure Refinement with SHELXL. Acta Crystallogr. Sect. C Struct. Chem. 2015, 71 (Md), 3-8.

(75) Steiner, T.; Koellner, G. Crystalline Beta-Cyclodextrin Hydrate at Various Humidities - Fast, Continuous, and Reversible Dehydration Studied by X-Ray-Diffraction. J. Am. Chem. Soc. 1994, 116 (12), 5122-5128.

(76) Haisa, M.; Kashino, S.; Kawai, R.; Maeda, H. The Monoclinic Form of P-Hydroxyacetanilide. Acta Crystallogr. Sect. B Struct. Crystallogr. Cryst. Chem. 1976, 58, 1283-1285.

(77) Smith, S. J.; Bishop, M. M.; Montgomery, J. M.; Hamilton, T. P.; Vohra, Y. K. Polymorphism in Paracetamol: Evidence of Additional Forms IV and v at High Pressure. J. Phys. Chem. A 2014, 118 (31), 6068-6077.

(78) Clegg, W. X-RAY CRYSTALLOGRAPHY; Oxford University Press; 2nd Revised edition (May 21, 2015), 2015.

(79) Chatjigakis, A. K.; Donzé, C.; Coleman, A. W.; Cardot, P. Solubility Behavior of BetaCyclodextrin In Water/Cosolvent Mixtures. Anal. Chem. 1992, 64, 1632-1634.

(80) Granberg, R. A.; Rasmuson, Å. C. Solubility of Paracetamol in Pure Solvents. J. Chem. Eng. Data 1999, 44, 1391-1395.

(81) Boldyreva, E. V.; Shakhtshneider, T. P.; Vasilchenko, M. a; Ahsbahs, H.; Uchtmann, H. Anisotropic Crystal Structure Distortion of the Monoclinic Polymorph of Acetaminophen at High Hydrostatic Pressures. Acta Crystallogr. B. 2000, 56 ( Pt 2), 299-309.

(82) Fabbiani, F. P. A.; Allan, D. R.; David, W. I. F.; Davidson, A. J.; Lennie, A. R.; Parsons, S.; Pulham, C. R.; Warren, J. E. High-Pressure Studies of Pharmaceuticals: An Exploration of the Behavior of Piracetam. Cryst. Growth Des. 2007, 7 (6), 1115-1124.

(83) Bergantin, S.; Moret, M.; Buth, G.; Fabbiani, F. P. A. Pressure-Induced Conformational Change in Organic Semiconductors: Triggering a Reversible Phase Transition in Rubrene. J. Phys. Chem. C 2014, 118, 13476-13483.

(84) Lübben, J.; Volkmann, C.; Grabowsky, S.; Edwards, A.; Morgenroth, W.; Fabbiani, F. P. a; Sheldrick, G. M.; Dittrich, B. On the Temperature Dependence of H-Uiso in the Riding Hydrogen Model. Acta Crystallogr. Sect. A Found. Adv. 2014, 70 (4), 309-316. 


\subsection{Appendix}

Table 4.5. RMSD values (Å) per $\beta$-CD dimer calculated using Crystal Packing Similarities in MERCURY. ${ }^{39}$

\begin{tabular}{|c|c|c|c|c|c|c|c|c|c|c|c|c|c|c|c|c|c|c|c|c|c|c|c|c|c|c|c|}
\hline & 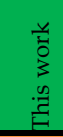 & 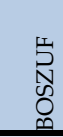 & 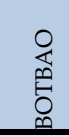 & 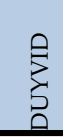 & 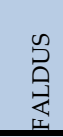 & 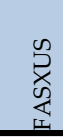 & 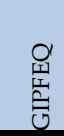 & 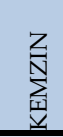 & 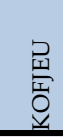 & 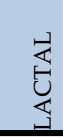 & 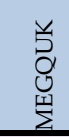 & $\begin{array}{l}\text { ज्ञ } \\
\text { N } \\
\text { m. } \\
3\end{array}$ & 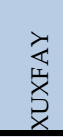 & 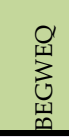 & 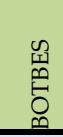 & 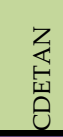 & 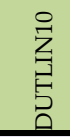 & 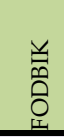 & 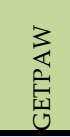 & 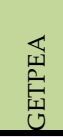 & $\begin{array}{l}\text { Q } \\
\mathbb{1} \\
\text { 至 } \\
\underline{\underline{1}} \\
\end{array}$ & $\begin{array}{l}3 \\
3 \\
3 \\
\Sigma\end{array}$ & 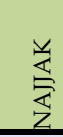 & 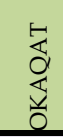 & 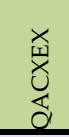 & 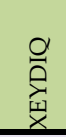 & 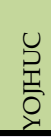 \\
\hline This work & & 2 & 2 & 2 & 2 & 2 & 2 & 2 & 2 & 2 & 2 & 2 & 2 & 2 & 2 & 1 & 2 & 2 & 2 & 2 & 2 & 2 & 2 & 2 & 2 & 2 & 2 \\
\hline BOSZUF & 0.12 & & 2 & 2 & 2 & 2 & 2 & 2 & 2 & 2 & 2 & 2 & 2 & 2 & 2 & 1 & 2 & 2 & 2 & 2 & 2 & 2 & 2 & 2 & 2 & 2 & 2 \\
\hline ВОТВАО & 0.15 & 0.16 & & 2 & 2 & 2 & 2 & 2 & 2 & 2 & 2 & 2 & 2 & 2 & 2 & 2 & 2 & 2 & 2 & 2 & 2 & 2 & 2 & 2 & 2 & 2 & 2 \\
\hline DUYVID & 0.12 & 0.04 & 0.15 & & 2 & 2 & 2 & 2 & 2 & 2 & 2 & 2 & 2 & 2 & 2 & 1 & 2 & 2 & 2 & 2 & 2 & 2 & 2 & 2 & 2 & 2 & 2 \\
\hline FALDUS & 0.12 & 0.10 & 0.19 & 0.10 & & 2 & 2 & 2 & 2 & 2 & 2 & 2 & 2 & 2 & 2 & 1 & 2 & 2 & 2 & 2 & 2 & 2 & 2 & 2 & 2 & 2 & 2 \\
\hline FASXUS & 0.13 & 0.06 & 0.17 & 0.05 & 0.11 & & 2 & 2 & 2 & 2 & 2 & 2 & 2 & 2 & 2 & 1 & 2 & 2 & 2 & 2 & 2 & 2 & 2 & 2 & 2 & 2 & 2 \\
\hline GIPFEQ & 0.11 & 0.06 & 0.19 & 0.07 & 0.08 & 0.08 & & 2 & 2 & 2 & 2 & 2 & 2 & 2 & 2 & 1 & 2 & 2 & 2 & 2 & 2 & 2 & 2 & 2 & 2 & 2 & 2 \\
\hline KEMZIN & 0.14 & 0.06 & 0.17 & 0.05 & 0.11 & 0.07 & 0.08 & & 2 & 2 & 2 & 2 & 2 & 2 & 2 & 1 & 2 & 2 & 2 & 2 & 2 & 2 & 2 & 2 & 2 & 2 & 2 \\
\hline KOFJEU & 0.11 & 0.06 & 0.18 & 0.04 & 0.09 & 0.07 & 0.07 & 0.07 & & 2 & 2 & 2 & 2 & 2 & 2 & 1 & 2 & 2 & 2 & 2 & 2 & 2 & 2 & 2 & 2 & 2 & 2 \\
\hline LACTAL & 0.11 & 0.07 & 0.19 & 0.06 & 0.09 & 0.07 & 0.05 & 0.08 & 0.05 & & 2 & 2 & 2 & 2 & 2 & 1 & 2 & 2 & 2 & 2 & 2 & 2 & 2 & 2 & 2 & 2 & 2 \\
\hline MEGQUK & 0.12 & 0.05 & 0.15 & 0.04 & 0.11 & 0.06 & 0.08 & 0.04 & 0.06 & 0.07 & & 2 & 2 & 2 & 2 & 1 & 2 & 2 & 2 & 2 & 2 & 2 & 2 & 2 & 2 & 2 & 2 \\
\hline WEZSIE & 0.12 & 0.05 & 0.17 & 0.04 & 0.10 & 0.06 & 0.06 & 0.07 & 0.04 & 0.05 & 0.05 & & 2 & 2 & 2 & 1 & 2 & 2 & 2 & 2 & 2 & 2 & 2 & 2 & 2 & 2 & 2 \\
\hline XUXFAY & 0.12 & 0.06 & 0.15 & 0.04 & 0.12 & 0.07 & 0.09 & 0.06 & 0.06 & 0.08 & 0.05 & 0.05 & & 2 & 2 & 1 & 2 & 2 & 2 & 2 & 2 & 2 & 2 & 2 & 2 & 2 & 2 \\
\hline BEGWEQ & 0.18 & 0.22 & 0.18 & 0.21 & 0.23 & 0.22 & 0.23 & 0.23 & 0.23 & 0.23 & 0.21 & 0.22 & 0.21 & & 2 & 1 & 2 & 2 & 2 & 2 & 2 & 2 & 2 & 2 & 2 & 2 & 2 \\
\hline BOTBES & 0.15 & 0.16 & 0.14 & 0.15 & 0.18 & 0.17 & 0.18 & 0.17 & 0.17 & 0.18 & 0.16 & 0.17 & 0.15 & 0.17 & & 1 & 2 & 2 & 2 & 2 & 2 & 2 & 2 & 2 & 2 & 2 & 2 \\
\hline CDETAN & 0.25 & 0.22 & 0.27 & 0.22 & 0.23 & 0.22 & 0.23 & 0.22 & 0.23 & 0.23 & 0.22 & 0.23 & 0.22 & 0.28 & 0.23 & & 1 & 1 & 1 & 2 & 1 & 1 & 1 & 1 & 1 & 2 & 1 \\
\hline DUTLIN10 & 0.17 & 0.19 & 0.10 & 0.19 & 0.21 & 0.20 & 0.21 & 0.20 & 0.21 & 0.21 & 0.19 & 0.21 & 0.18 & 0.17 & 0.10 & 0.24 & & 2 & 2 & 2 & 2 & 2 & 2 & 2 & 2 & 2 & 2 \\
\hline FODBIK & 0.14 & 0.12 & 0.19 & 0.11 & 0.14 & 0.12 & 0.13 & 0.13 & 0.11 & 0.12 & 0.12 & 0.11 & 0.12 & 0.21 & 0.11 & 0.24 & 0.17 & & 2 & 2 & 2 & 2 & 2 & 2 & 2 & 2 & 2 \\
\hline GETPAW & 0.17 & 0.18 & 0.09 & 0.17 & 0.20 & 0.19 & 0.20 & 0.18 & 0.20 & 0.21 & 0.17 & 0.19 & 0.17 & 0.18 & 0.12 & 0.24 & 0.08 & 0.18 & & 2 & 2 & 2 & 2 & 2 & 2 & 2 & 2 \\
\hline GETPEA & 0.20 & 0.23 & 0.12 & 0.22 & 0.24 & 0.24 & 0.24 & 0.24 & 0.25 & 0.25 & 0.22 & 0.24 & 0.21 & 0.20 & 0.13 & 0.31 & 0.08 & 0.21 & 0.11 & & 2 & 2 & 2 & 2 & 2 & 2 & 2 \\
\hline KIFPAQ & 0.13 & 0.06 & 0.17 & 0.04 & 0.10 & 0.06 & 0.07 & 0.06 & 0.05 & 0.06 & 0.06 & 0.05 & 0.06 & 0.21 & 0.17 & 0.22 & 0.20 & 0.12 & 0.19 & 0.24 & & 2 & 2 & 2 & 2 & 2 & 2 \\
\hline MACDAW & 0.15 & 0.14 & 0.22 & 0.13 & 0.15 & 0.13 & 0.14 & 0.14 & 0.13 & 0.12 & 0.14 & 0.13 & 0.14 & 0.21 & 0.14 & 0.24 & 0.21 & 0.06 & 0.21 & 0.25 & 0.13 & & 2 & 2 & 2 & 2 & 2 \\
\hline NAJJAK & 0.14 & 0.16 & 0.15 & 0.15 & 0.17 & 0.16 & 0.17 & 0.16 & 0.16 & 0.17 & 0.15 & 0.16 & 0.15 & 0.14 & 0.14 & 0.23 & 0.14 & 0.15 & 0.14 & 0.17 & 0.15 & 0.16 & & 2 & 2 & 2 & 2 \\
\hline OKAQAT & 0.15 & 0.15 & 0.17 & 0.14 & 0.15 & 0.14 & 0.15 & 0.15 & 0.15 & 0.15 & 0.15 & 0.15 & 0.13 & 0.20 & 0.13 & 0.25 & 0.17 & 0.09 & 0.17 & 0.20 & 0.15 & 0.13 & 0.17 & & 2 & 2 & 2 \\
\hline QACXEX & 0.16 & 0.15 & 0.20 & 0.14 & 0.16 & 0.14 & 0.15 & 0.15 & 0.14 & 0.15 & 0.14 & 0.14 & 0.14 & 0.19 & 0.11 & 0.23 & 0.17 & 0.08 & 0.18 & 0.21 & 0.14 & 0.07 & 0.13 & 0.13 & & 2 & 2 \\
\hline XEYDIQ & 0.13 & 0.12 & 0.09 & 0.12 & 0.15 & 0.13 & 0.15 & 0.12 & 0.14 & 0.15 & 0.11 & 0.13 & 0.11 & 0.18 & 0.09 & 0.25 & 0.10 & 0.12 & 0.08 & 0.14 & 0.13 & 0.15 & 0.12 & 0.13 & 0.13 & & 2 \\
\hline YOJHUC & 0.17 & 0.21 & 0.17 & 0.21 & 0.22 & 0.21 & 0.21 & 0.21 & 0.21 & 0.21 & 0.21 & 0.21 & 0.21 & 0.13 & 0.18 & 0.28 & 0.17 & 0.21 & 0.16 & 0.18 & 0.21 & 0.19 & 0.13 & 0.21 & 0.18 & 0.17 & \\
\hline
\end{tabular}


Table 4.6. PXRD similarities of all C2221 and P21 structures studied in this chapter calculated using Crystal Packing Similarities in MERCURY

\begin{tabular}{|c|c|c|c|c|c|c|c|c|c|c|c|c|c|c|c|c|c|c|c|c|c|c|c|c|c|c|c|}
\hline & 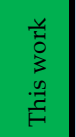 & 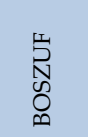 & 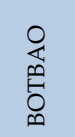 & $\sum_{\substack{5 \\
0}}^{0}$ & 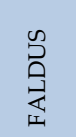 & 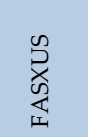 & 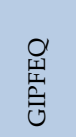 & 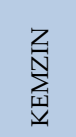 & 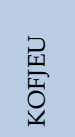 & 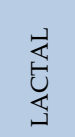 & 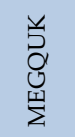 & 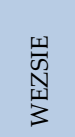 & 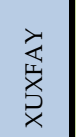 & 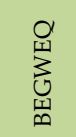 & $\begin{array}{l}\text { 岱 } \\
\stackrel{0}{0} \\
0\end{array}$ & 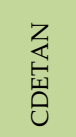 & $\begin{array}{l}\stackrel{ }{\vec{Z}} \\
\overrightarrow{3} \\
\vec{B} \\
\stackrel{0}{0}\end{array}$ & 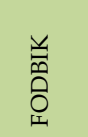 & 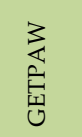 & 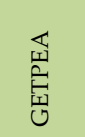 & 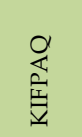 & 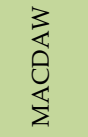 & 壱 & $\begin{array}{l}\text { 离 } \\
\frac{1}{\mathbb{1}} \\
\text { o }\end{array}$ & 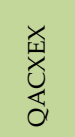 & 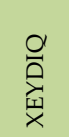 & 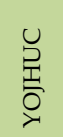 \\
\hline \multicolumn{28}{|l|}{ This work } \\
\hline BOSZUF & 0.989 & & & & & & & & & & & & & & & & & & & & & & & & & & \\
\hline ВОТВАО & 0.994 & 0.997 & & & & & & & & & & & & & & & & & & & & & & & & & \\
\hline DUYVID & 0.991 & 0.998 & 0.998 & & & & & & & & & & & & & & & & & & & & & & & & \\
\hline FALDUS & 0.993 & 0.998 & 0.997 & 0.998 & & & & & & & & & & & & & & & & & & & & & & & \\
\hline FASXUS & 0.992 & 0.998 & 0.999 & 1.000 & 0.998 & & & & & & & & & & & & & & & & & & & & & & \\
\hline GIPFEQ & 0.992 & 0.999 & 0.998 & 0.999 & 0.999 & 0.999 & & & & & & & & & & & & & & & & & & & & & \\
\hline KEMZIN & 0.991 & 0.999 & 0.998 & 1.000 & 0.998 & 1.000 & 0.999 & & & & & & & & & & & & & & & & & & & & \\
\hline KOFJEU & 0.993 & 0.996 & 0.997 & 0.999 & 0.997 & 0.998 & 0.998 & 0.997 & & & & & & & & & & & & & & & & & & & \\
\hline LACTAL & 0.994 & 0.997 & 0.998 & 0.999 & 0.998 & 0.998 & 0.999 & 0.998 & 1.000 & & & & & & & & & & & & & & & & & & \\
\hline MEGQUK & 0.992 & 0.998 & 0.999 & 1.000 & 0.998 & 1.000 & 0.999 & 1.000 & 0.998 & 0.998 & & & & & & & & & & & & & & & & & \\
\hline WEZSIE & 0.990 & 0.998 & 0.996 & 1.000 & 0.997 & 0.999 & 0.999 & 0.999 & 0.998 & 0.998 & 0.999 & & & & & & & & & & & & & & & & \\
\hline XUXFAY & 0.990 & 0.999 & 0.997 & 1.000 & 0.997 & 0.999 & 0.999 & 1.000 & 0.997 & 0.998 & 0.999 & 0.999 & & & & & & & & & & & & & & & \\
\hline BEGWEQ & 0.972 & 0.989 & 0.982 & 0.988 & 0.985 & 0.985 & 0.987 & 0.987 & 0.986 & 0.985 & 0.986 & 0.989 & 0.989 & & & & & & & & & & & & & & \\
\hline BOTBES & 0.980 & 0.992 & 0.987 & 0.991 & 0.988 & 0.989 & 0.990 & 0.991 & 0.989 & 0.988 & 0.990 & 0.992 & 0.992 & 0.996 & & & & & & & & & & & & & \\
\hline CDETAN & 0.986 & 0.993 & 0.991 & 0.995 & 0.992 & 0.994 & 0.994 & 0.994 & 0.995 & 0.994 & 0.995 & 0.996 & 0.996 & 0.991 & 0.994 & & & & & & & & & & & & \\
\hline DUTLIN10 & 0.988 & 0.994 & 0.993 & 0.995 & 0.992 & 0.995 & 0.993 & 0.995 & 0.993 & 0.993 & 0.995 & 0.996 & 0.996 & 0.991 & 0.996 & 0.998 & & & & & & & & & & & \\
\hline FODBIK & 0.983 & 0.991 & 0.987 & 0.993 & 0.989 & 0.991 & 0.991 & 0.992 & 0.993 & 0.992 & 0.992 & 0.994 & 0.993 & 0.993 & 0.996 & 0.999 & 0.997 & & & & & & & & & & \\
\hline GETPAW & 0.991 & 0.992 & 0.994 & 0.995 & 0.992 & 0.995 & 0.993 & 0.995 & 0.995 & 0.994 & 0.996 & 0.995 & 0.994 & 0.985 & 0.991 & 0.996 & 0.998 & 0.995 & & & & & & & & & \\
\hline GETPEA & 0.987 & 0.995 & 0.993 & 0.995 & 0.992 & 0.994 & 0.994 & 0.995 & 0.993 & 0.993 & 0.995 & 0.996 & 0.996 & 0.992 & 0.997 & 0.997 & 1.000 & 0.996 & 0.997 & & & & & & & & \\
\hline KIFPAQ & 0.990 & 0.998 & 0.996 & 1.000 & 0.997 & 0.999 & 0.999 & 0.999 & 0.999 & 0.999 & 0.999 & 1.000 & 0.999 & 0.989 & 0.991 & 0.996 & 0.995 & 0.994 & 0.995 & 0.995 & & & & & & & \\
\hline MACDAW & 0.978 & 0.988 & 0.984 & 0.990 & 0.986 & 0.988 & 0.988 & 0.989 & 0.990 & 0.989 & 0.989 & 0.992 & 0.991 & 0.995 & 0.996 & 0.997 & 0.995 & 0.999 & 0.992 & 0.995 & 0.992 & & & & & & \\
\hline NAJJAK & 0.987 & 0.991 & 0.990 & 0.995 & 0.990 & 0.993 & 0.992 & 0.994 & 0.995 & 0.993 & 0.994 & 0.995 & 0.994 & 0.991 & 0.994 & 0.999 & 0.998 & 0.999 & 0.998 & 0.997 & 0.995 & 0.997 & & & & & \\
\hline OKAQAT & 0.980 & 0.991 & 0.986 & 0.991 & 0.988 & 0.989 & 0.990 & 0.991 & 0.990 & 0.989 & 0.990 & 0.992 & 0.992 & 0.996 & 0.999 & 0.996 & 0.996 & 0.998 & 0.992 & 0.997 & 0.992 & 0.998 & 0.996 & & & & \\
\hline QACXEX & 0.977 & 0.989 & 0.984 & 0.990 & 0.986 & 0.988 & 0.988 & 0.990 & 0.988 & 0.987 & 0.989 & 0.991 & 0.991 & 0.997 & 0.999 & 0.995 & 0.996 & 0.997 & 0.991 & 0.996 & 0.991 & 0.998 & 0.996 & 1.000 & & & \\
\hline XEYDIQ & 0.989 & 0.992 & 0.992 & 0.995 & 0.991 & 0.995 & 0.993 & 0.995 & 0.995 & 0.994 & 0.995 & 0.996 & 0.995 & 0.988 & 0.994 & 0.998 & 0.999 & 0.998 & 0.999 & 0.998 & 0.996 & 0.995 & 0.999 & 0.995 & 0.994 & & \\
\hline YOJHUC & 0.984 & 0.989 & 0.987 & 0.991 & 0.988 & 0.990 & 0.989 & 0.990 & 0.991 & 0.990 & 0.991 & 0.992 & 0.991 & 0.990 & 0.993 & 0.996 & 0.997 & 0.997 & 0.996 & 0.996 & 0.992 & 0.996 & 0.998 & 0.995 & 0.995 & 0.997 & \\
\hline
\end{tabular}




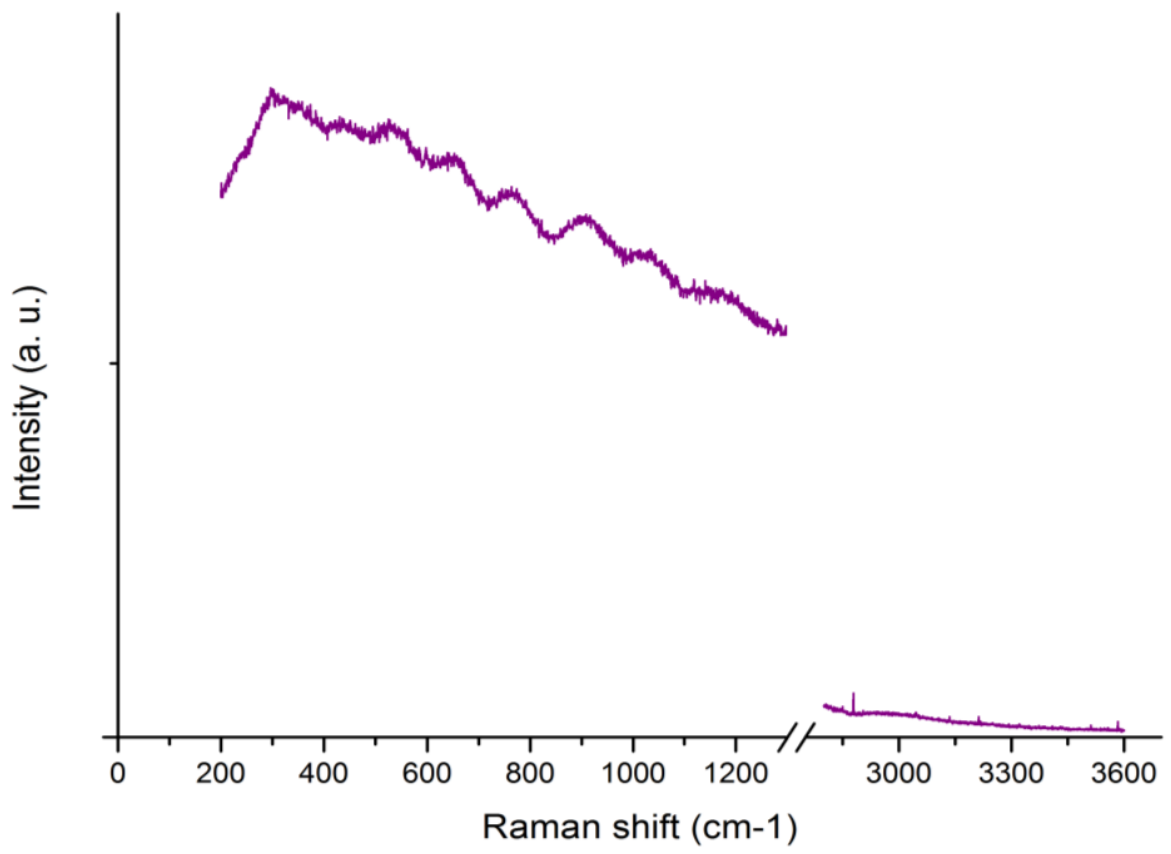

Figure 4.18. Raman spectrum of the solution surrounding the crystal of $\beta$-CD.paracetamol form $V$.

Spectrum presented as obtained without any treatment

Table 4.7. Calculation of the average non-H atomic volume

\begin{tabular}{|c|c|c|c|c|c|c|c|c|c|}
\hline & $\mathrm{Z}\left(\mathrm{Z}^{\prime}\right)$ & $\begin{array}{c}\beta-C D \\
\text { formula }\end{array}$ & Guest's formula & $\begin{array}{c}\text { non-H } \\
\text { count } \\
\text { (Guest) }\end{array}$ & $\mathrm{H}_{2} \mathrm{O}$ & non-H count & $\begin{array}{c}\mathrm{V} \\
/ \AA^{3}\end{array}$ & $\begin{array}{l}\mathrm{V} / \mathrm{Z} \\
/ \AA^{3}\end{array}$ & $\begin{array}{c}\text { Atomic } \\
\text { volume } \\
/ \AA^{3}\end{array}$ \\
\hline This work & $8(1)$ & $\mathrm{C}_{42} \mathrm{H}_{70} \mathrm{O}_{34}$ & $0.3\left(\mathrm{C}_{8} \mathrm{H}_{9} \mathrm{NO}_{2}\right)$ & 3.3 & 24.15 & 104.45 & 15669.45 & 1958.6 & 18.75 \\
\hline BOSZUF & $8(1)$ & $\mathrm{C}_{42} \mathrm{H}_{70} \mathrm{O}_{34}$ & $\mathrm{C}_{10} \mathrm{H}_{8} \mathrm{~N}_{2}$ & 12 & 13.50 & 102.50 & 14937.35 & 1867.1 & 18.22 \\
\hline ВОТВАО & $8(1)$ & $\mathrm{C}_{42} \mathrm{H}_{70} \mathrm{O}_{34}$ & $\mathrm{C}_{12} \mathrm{H}_{10} \mathrm{~N}_{2}$ & 14 & 10.50 & 101.50 & 15119.05 & 1889.8 & 18.62 \\
\hline DUYVID & $8(1)$ & $\mathrm{C}_{42} \mathrm{H}_{70} \mathrm{O}_{34}$ & $1.5\left(\mathrm{C}_{10} \mathrm{H}_{16}\right)$ & 15 & 10.10 & 102.10 & 15032.64 & 1879.0 & 18.40 \\
\hline FALDUS & $8(1)$ & $\mathrm{C}_{42} \mathrm{H}_{70} \mathrm{O}_{34}$ & $\mathrm{C}_{6} \mathrm{H}_{14} \mathrm{O}_{2}$ & 8 & 9.00 & 94.00 & 15428.19 & 1928.5 & 20.52 \\
\hline FASXUS & $8(1)$ & $\mathrm{C}_{42} \mathrm{H}_{70} \mathrm{O}_{34}$ & $\mathrm{C}_{11} \mathrm{H}_{18} \mathrm{O}$ & 12 & 11.00 & 100.00 & 14969.10 & 1871.1 & 18.71 \\
\hline GIPFEQ & $8(1)$ & $\mathrm{C}_{42} \mathrm{H}_{70} \mathrm{O}_{34}$ & $\mathrm{C}_{3} \mathrm{H}_{12} \mathrm{Cl}_{2} \mathrm{NPPt}$ & 8 & 5.50 & 90.50 & 15209.36 & 1901.1 & 21.01 \\
\hline KEMZIN & $8(1)$ & $\mathrm{C}_{42} \mathrm{H}_{70} \mathrm{O}_{34}$ & $\mathrm{C}_{10} \mathrm{H}_{16}$ & 10 & 10.00 & 97.00 & 14930.60 & 1866.3 & 19.24 \\
\hline KOFJEU & $8(1)$ & $\mathrm{C}_{42} \mathrm{H}_{70} \mathrm{O}_{34}$ & $\mathrm{C}_{11} \mathrm{H}_{16} \mathrm{O}$ & 12 & 10.00 & 99.00 & 15362.28 & 1920.2 & 19.40 \\
\hline LACTAL & $8(1)$ & $\mathrm{C}_{42} \mathrm{H}_{70} \mathrm{O}_{34}$ & $\mathrm{C}_{14} \mathrm{H}_{16} \mathrm{~N}_{2}$ & 16 & 8.00 & 101.00 & 15401.57 & 1925.2 & 19.06 \\
\hline MEGQUK & $8(1)$ & $\mathrm{C}_{42} \mathrm{H}_{70} \mathrm{O}_{34}$ & $\mathrm{C}_{10} \mathrm{H}_{14} \mathrm{~N}_{2} \mathrm{O}$ & 13 & 10.50 & 100.50 & 14979.15 & 1872.3 & 18.63 \\
\hline WEZSIE & $8(1)$ & $\mathrm{C}_{42} \mathrm{H}_{70} \mathrm{O}_{34}$ & $0.5\left(\mathrm{C}_{14} \mathrm{H}_{12} \mathrm{O}_{3 \mathrm{~s}}\right)$ & 9 & 10.00 & 96.00 & 14959.29. & 1869.9 & 19.48 \\
\hline XUXFAY & $8(1)$ & $\mathrm{C}_{42} \mathrm{H}_{70} \mathrm{O}_{34}$ & $\mathrm{C}_{11} \mathrm{H}_{16} \mathrm{O}_{52}$ & 14 & 12.50 & 103.50 & 14936.90 & 1867.1 & 18.04 \\
\hline BOTBES & $4(2)$ & $\mathrm{C}_{42} \mathrm{H}_{70} \mathrm{O}_{34}$ & ${ }_{12} \mathrm{H}_{10} \mathrm{~N}_{2}, 0.25\left(\mathrm{CH}_{4} \mathrm{O}\right.$ & 14.5 & 11.25 & 102.75 & 7278.9 & 1819.7 & 17.71 \\
\hline BEGWEQ & $4(2)$ & $\mathrm{C}_{42} \mathrm{H}_{70} \mathrm{O}_{34}$ & $0.5\left(\mathrm{C}_{19} \mathrm{H}_{20} \mathrm{FNO}_{3}\right)$ & 12 & 14.00 & 103.00 & 7278.7 & 1819.6 & 17.67 \\
\hline CDETAN & $4(2)$ & $\mathrm{C}_{42} \mathrm{H}_{70} \mathrm{O}_{34}$ & $\mathrm{C}_{8} \mathrm{H}_{11} \mathrm{~N}$ & 9 & 16.00 & 102.00 & 7524.3 & 1881.0 & 18.44 \\
\hline DUTLIN10 & $4(2)$ & $\mathrm{C}_{42} \mathrm{H}_{70} \mathrm{O}_{34}$ & $\mathrm{C}_{15} \mathrm{H}_{14} \mathrm{O}_{3}$ & 18 & 12.50 & 107.50 & 7398.6 & 1849.6 & 17.21 \\
\hline FODBIK & $4(2)$ & $\mathrm{C}_{42} \mathrm{H}_{70} \mathrm{O}_{34}$ & $\mathrm{C}_{12} \mathrm{H}_{11} \mathrm{~N}_{3}$ & 15 & 10.75 & 102.75 & 7523.3 & 1880.8 & 18.30 \\
\hline KIFPAQ & $4(2)$ & $\mathrm{C}_{42} \mathrm{H}_{70} \mathrm{O}_{34}$ & $\mathrm{C}_{10} \mathrm{H}_{14} \mathrm{O}$ & 11 & 7.25 & 95.25 & 7534.9 & 1883.7 & 19.78 \\
\hline MACDAW & $4(2)$ & $\mathrm{C}_{42} \mathrm{H}_{70} \mathrm{O}_{34}$ & $\mathrm{C}_{12} \mathrm{H}_{12} \mathrm{~N}_{2}$ & 14 & 12.35 & 103.35 & 7473.7 & 1868.4 & 18.08 \\
\hline NAJJAK & $4(2)$ & $\mathrm{C}_{42} \mathrm{H}_{70} \mathrm{O}_{34}$ & $1.5\left(\mathrm{C}_{8} \mathrm{H}_{62} \mathrm{~s}_{2}\right)$ & 15 & 10.50 & 102.50 & 7533.4 & 1883.3 & 18.37 \\
\hline OKAQAT & $4(2)$ & $\mathrm{C}_{42} \mathrm{H}_{70} \mathrm{O}_{34}$ & $\mathrm{C}_{15} \mathrm{H}_{11} \mathrm{~N}$ & 16 & 9.13 & 102.13 & 7375.0 & 1843.7 & 18.05 \\
\hline QACXEX & $4(2)$ & $\mathrm{C}_{42} \mathrm{H}_{70} \mathrm{O}_{34}$ & $\mathrm{C}_{12} \mathrm{H}_{10} \mathrm{~N}_{2} \mathrm{O}_{2}$ & 16 & 12.75 & 105.75 & 7282.4 & 1820.6 & 17.22 \\
\hline XEYDIQ & $4(2)$ & $\mathrm{C}_{42} \mathrm{H}_{70} \mathrm{O}_{34}$ & $\mathrm{C}_{10} \mathrm{H}_{20} \mathrm{O}$ & 11 & 13.50 & 101.50 & 7486.5 & 1871.6 & 18.44 \\
\hline YOJHUC & $4(2)$ & $\mathrm{C}_{42} \mathrm{H}_{70} \mathrm{O}_{34}$ & $\mathrm{C}_{12} \mathrm{H}_{12} \mathrm{~N}_{2} \mathrm{~S}_{2}$ & 16 & 11.75 & 104.75 & 7369.5 & 1842.3 & 17.59 \\
\hline
\end{tabular}


Table 4.8. Effect of Pressure and temperature on the average atomic volume

\begin{tabular}{|c|c|c|c|c|c|c|c|}
\hline Compound & CSD Refcode & $\mathrm{P} / \mathrm{GPa}$ & $\mathrm{T} / \mathrm{K}$ & $\mathrm{V} / \AA^{3}$ & Z & non-H count & $\begin{array}{c}\text { Atomic } \\
\text { volume } \\
/ \AA^{3}\end{array}$ \\
\hline \multirow{4}{*}{ Paracetamol $^{81}$} & HXACAN09 & 1.00 & $\mathrm{RT}$ & 711.734 & 4 & 11 & 16.18 \\
\hline & HXACAN10 & 2.00 & $\mathrm{RT}$ & 672.012 & 4 & 11 & 15.27 \\
\hline & HXACAN11 & 3.00 & RT & 651.428 & 4 & 11 & 14.81 \\
\hline & HXACAN12 & 4.00 & $\mathrm{RT}$ & 621.881 & 4 & 11 & 14.13 \\
\hline \multirow{5}{*}{ Piracetam $^{82}$} & BISMEV06 & 0.45 & RT & 334.013 & 2 & 10 & 16.70 \\
\hline & BISMEV07 & 0.70 & RT & 325.534 & 2 & 10 & 16.28 \\
\hline & BISMEV08 & 0.90 & RT & 314.262 & 2 & 10 & 15.71 \\
\hline & BISMEV09 & 2.50 & RT & 292.826 & 2 & 10 & 14.64 \\
\hline & BISMEV10 & 4.00 & RT & 280.206 & 2 & 10 & 14.01 \\
\hline \multirow{8}{*}{ Ruberene $^{83}$} & / & $\mathrm{AP}$ & RT & 697.9 & 1 & 42 & 16.62 \\
\hline & l & 0.15 & RT & 687.2 & 1 & 42 & 16.36 \\
\hline & / & 1.21 & RT & 633 & 1 & 42 & 15.07 \\
\hline & I & 2.42 & RT & 598.4 & 1 & 42 & 14.25 \\
\hline & / & 3.58 & RT & 577.6 & 1 & 42 & 13.75 \\
\hline & I & 4.65 & RT & 559 & 1 & 42 & 13.31 \\
\hline & I & 5.91 & $\mathrm{RT}$ & 542.8 & 1 & 42 & 12.92 \\
\hline & I & 7.12 & $\mathrm{RT}$ & 1040.3 & 2 & 42 & 12.38 \\
\hline \multirow{8}{*}{$\begin{array}{c}\text { N-Acetyl-L- } \\
\text { hydroxyproline } \\
\text { hy }\end{array}$} & POKKAD05 & $\mathrm{AP}$ & 9 & 924.5 & 4 & 13 & 17.78 \\
\hline & POKKAD06 & $\mathrm{AP}$ & 30 & 924.7 & 4 & 13 & 17.78 \\
\hline & POKKAD07 & $\mathrm{AP}$ & 50 & 926.2 & 4 & 13 & 17.81 \\
\hline & POKKAD08 & $\mathrm{AP}$ & 75 & 928.7 & 4 & 13 & 17.86 \\
\hline & POKKAD09 & $\mathrm{AP}$ & 100 & 931.06 & 4 & 13 & 17.91 \\
\hline & POKKAD10 & $\mathrm{AP}$ & 150 & 936.55 & 4 & 13 & 18.01 \\
\hline & POKKAD11 & $\mathrm{AP}$ & 200 & 941.99 & 4 & 13 & 18.12 \\
\hline & POKKAD & $\mathrm{AP}$ & 250 & 949.32 & 4 & 13 & 18.26 \\
\hline
\end{tabular}




\section{Chapter 5. Screening of inclusion complex formation at ambient- and high- pressure conditions}

\subsection{Synopsis}

This chapter summarises the majority of the experiments conducted to explore inclusion complex formation at ambient- and high-pressure conditions, using $\alpha$-, $\beta$ - and hydroxypropyl- $\beta$ cyclodextrins together with several organic molecules. The original idea was to induce complex formation by applying pressure; however our experiments have quickly shown that water solubility of cyclodextrins increases drastically as function of pressure, thus a "reverse" mechanism, where guest molecules crystallise out of $\mathrm{CD}$ cavities at high pressure, takes place. A new route for inclusion complex formation in solution at high pressure that exploits the observed increased solubility trend of $\mathrm{CD}$ is described herein.

\subsection{Introduction}

High-pressure crystallisation techniques proved to be a powerful means of investigating drug polymorphism in binary (solute/solvent) systems. ${ }^{1-5}$ Our high-pressure studies on the tertiary $\beta$-CD.paracetamol'water system (see Chapter 4 ) give reason to assume that by applying pressure, water molecules partially drive the guest molecules out of the cyclodextrins cavities, giving a guest-deficient complex and crystals of the guest molecules alone. In order to further support and investigate this theory, more experiments were conducted using different organic molecules. The scope of these studies was to first explore inclusion complex formation at ambient conditions, and then, if complex formation was successful, explore the solid-state behaviour of the complex at high pressure. The second aim was to use high-pressure techniques directly to investigate inclusion complex formation from supersaturated solutions by in situ crystallisation. The screening was carried out using $\alpha$-, $\beta$ - and hydroxypropyl- $\beta$-cyclodextrin. The latter is a derivative of $\beta$ cyclodextrin. Inclusion complex formation was investigated on the following compounds:

> aspartame, an artificial dipeptide sweetener serendipitously discovered in 1965 and used worldwide as artificial sweetener to control conditions, such as obesity, caused by glucose; 6

$>$ gabapentin, an analogue of $\gamma$-aminobutyric acid, used as an anticonvulsant and analgesic drug; ${ }^{7}$

$>$ L-dopa, a pre-neurotransmitter used for treating Parkinson's disease; ${ }^{8}$

$>$ piracetam, a nootropic drug used as cognitive enhancer; 9

$>$ 5-fluorouracil (5FU), an anti-cancer drug; ${ }^{10}$

$>$ L-ascorbic acid, commonly known as vitamin C, an antioxidant agent; ${ }^{11}$ 
ampicillin and amoxicillin, broad bacterial antibiotics of the penicillin's family; ${ }^{12}$

$>$ and finally 6-aminopenicillanic acid (6-APA), a natural compound constituting the core of penicillins and used in their semisynthesis. ${ }^{13}$

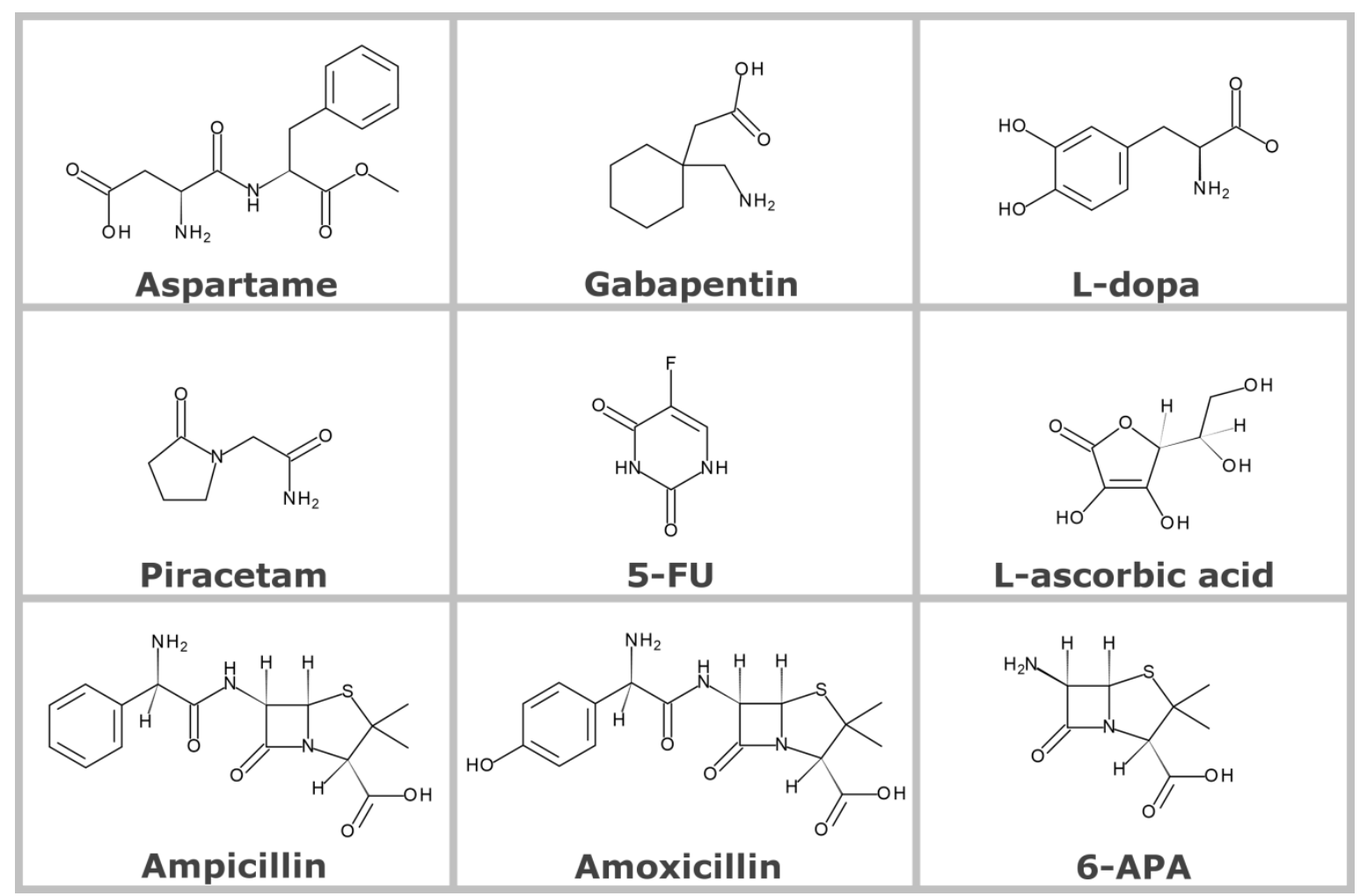

Figure 5.1. Chemical diagrams of the investigated compounds.

\subsection{Experimental}

\subsubsection{Material}

All compounds were purchased from Sigma-Aldrich and used as supplied without further treatment. Approximate water solubility values are listed in Table 5.1.

Table 5.1. List of the studied compounds and their water solubility values.

\begin{tabular}{|ccc|}
\hline Compound & Solubility in water $(\mathrm{g} / \mathrm{L})$ & $\begin{array}{c}\text { Temperature } \\
\left({ }^{\circ} \mathbf{C}\right)\end{array}$ \\
\hline $\boldsymbol{\alpha}$-CD & $145^{14}$ & RT \\
$\beta$-CD & $18.5^{14}$ & RT \\
hp- $\beta$-CD & $2300^{15}$ & 24 \\
\hline 6-APA & $2.46^{16}$ & 20 \\
\hline Amoxicillin & $3.430^{17}$ & not specified \\
\hline Ampicillin & $10.1^{17}$ & 21 \\
\hline Aspartame & $10.5^{18}$ & 25 \\
\hline Carbamazepine & $0.0177^{17}$ & not specified \\
\hline 5FU & $11.1^{17}$ & 22 \\
\hline Gabapentin & $4.49^{17}$ & not specified \\
\hline L-ascorbic acid & $400^{17}$ & 40 \\
\hline L-dopa & $5^{17}$ & 20 \\
\hline Piracetam & $72^{19}$ & 25 \\
\hline Succinic acid & $83.2^{17}$ & 25 \\
\hline
\end{tabular}




\subsubsection{Crystallisation at ambient conditions}

A screening of inclusion complex formation was carried out using $\alpha_{-}, \beta$ - and hydroxypropyl- $\beta$-CDs. Approximately equimolar aqueous solutions of each CD with a drug molecule were prepared with a slight excess of the drug molecule to allow maximisation of the number of filled CD-cavities in case of complex formation. All experiments are listed in Table 5.2. Additionally, reference vials of aqueous solutions of $\mathrm{CD}$ and drug molecules as single components were prepared to compare microscopically the outcome of the co-crystallisation experiments. After dissolving both compounds in demineralised water, the solutions were heated up to $353 \mathrm{~K}$ to dissolve any remaining solid. The inclusion complex formation was investigated using the slow evaporation method at room temperature and crystallisation was monitored using polarised optical microscopy.

Table 5.2. Summary of the experimental inclusion complex screening at ambient-pressure condition.

\begin{tabular}{|c|c|c|c|}
\hline & in $\alpha$-CD'water & in $\beta$-CD.water & in hp- $\beta$-CD water \\
\hline Carbamazepine & $\begin{array}{l}\text { Carbamazepine is poorly soluble; fast } \\
\text { evaporation leads to the precipitation } \\
\text { of amorphous material }\end{array}$ & $\begin{array}{l}\text { Carbamazepine is poorly soluble - } \\
\text { heating induces the complete } \\
\text { dissolution, fast crystallisation } \\
\text { observed after the heat source is off }\end{array}$ & $\begin{array}{l}\text { Carbamazepine is poorly soluble - } \\
\text { heating induces the complete } \\
\text { dissolution, fast crystallisation } \\
\text { observed after the heat source is off }\end{array}$ \\
\hline Aspartame & $\begin{array}{c}\text { Aspartame forms aggregates and does } \\
\text { not completely dissolve }\end{array}$ & $\begin{array}{c}\text { Aspartame forms aggregates - heating } \\
\text { induces dissolution; crystallisation } \\
\text { observed on cooling }\end{array}$ & $\begin{array}{c}\text { Aspartame forms aggregates and does } \\
\text { not completely dissolve }\end{array}$ \\
\hline $5 \mathrm{FU}$ & $\begin{array}{c}\text { Solubility of } 5 \mathrm{FU} \text { increases markedly } \\
\text { and crystallisation of large crystals is } \\
\text { observed }\end{array}$ & $\begin{array}{c}\text { Solubility of } 5 \mathrm{FU} \text { increases markedly } \\
\text { and crystallisation of large crystals is } \\
\text { observed }\end{array}$ & $\begin{array}{l}\text { This combination has not been } \\
\text { investigated }\end{array}$ \\
\hline 6-APA & $\begin{array}{c}\alpha \text {-CD does not improve 6-APA water } \\
\text { solubility, and does not prevent its } \\
\text { degradation - No evidence of } \\
\text { crystallisation }\end{array}$ & $\begin{array}{c}\beta \text {-CD does not improve 6-APA water } \\
\text { solubility, and does not prevent its } \\
\text { degradation - No evidence of } \\
\text { crystallisation }\end{array}$ & $\begin{array}{c}\text { Hp- } \beta \text {-CD does not improve 6-APA } \\
\text { water solubility, and does not prevent } \\
\text { its degradation - No evidence of } \\
\text { crystallisation }\end{array}$ \\
\hline Amoxicillin & $\begin{array}{l}\alpha \text {-CD does not improve amoxicillin } \\
\text { water solubility, and does not prevent } \\
\text { its degradation - No evidence of } \\
\text { crystallisation }\end{array}$ & $\begin{array}{c}\beta \text {-CD does not improve amoxicillin } \\
\text { water solubility, and does not prevent } \\
\text { its degradation - No evidence of } \\
\text { crystallisation }\end{array}$ & $\begin{array}{c}\text { Hp- } \beta \text {-CD does not improve amoxicillin } \\
\text { water solubility, and does not prevent } \\
\text { its degradation - No evidence of } \\
\text { crystallisation }\end{array}$ \\
\hline Ampicillin & $\begin{array}{c}\text { This combination has not been } \\
\text { investigated }\end{array}$ & $\begin{array}{c}\text { This combination has not been } \\
\text { investigated }\end{array}$ & $\begin{array}{l}\text { Hp- } \beta \text {-CD improves slightly the water } \\
\text { solubility of ampicillin however } \\
\text { ampicillin remains unstable }\end{array}$ \\
\hline Gabapentin & $\begin{array}{l}\text { Complete dissolution and } \\
\text { crystallisation after slow evaporation }\end{array}$ & $\begin{array}{l}\text { Complete dissolution and } \\
\text { crystallisation after slow evaporation }\end{array}$ & No evidence of crystallisation \\
\hline L-dopa & $\begin{array}{l}\text { This combination has not been } \\
\text { investigated }\end{array}$ & $\begin{array}{l}\text { Complete dissolution and } \\
\text { crystallisation of two populations } \\
\text { observed after slow evaporation - L- } \\
\text { dopa is unstable and the solutions } \\
\text { deteriorates }\end{array}$ & $\begin{array}{l}\text { This combination has not been } \\
\text { investigated }\end{array}$ \\
\hline Piracetam & $\begin{array}{l}\text { This combination has not been } \\
\text { investigated }\end{array}$ & $\begin{array}{l}\text { Complete dissolution and } \\
\text { crystallisation after slow evaporation }\end{array}$ & $\begin{array}{l}\text { This combination has not been } \\
\text { investigated }\end{array}$ \\
\hline Succinic acid & See Chapter 3 & $\begin{array}{l}\text { Complete dissolution and } \\
\text { crystallisation after slow evaporation }\end{array}$ & $\begin{array}{l}\text { This combination has not been } \\
\text { investigated }\end{array}$ \\
\hline L-ascorbic acid & $\begin{array}{l}\text { Complete dissolution and glass } \\
\text { formation after slow evaporation }\end{array}$ & $\begin{array}{l}\text { Complete dissolution and } \\
\text { crystallisation after slow evaporation }\end{array}$ & No evidence of crystallisation \\
\hline
\end{tabular}

\subsubsection{Crystallisation in a DAC}

For the high-pressure experiments, different types of DAC were used: a beryllium-free modified Merrill-Bassett DAC, ${ }^{20,21}$ a beryllium-free DAC of the Ahsbahs type, ${ }^{22}$ a rectangularshaped DAC and a round-shaped DAC both equipped with Beryllium backing plates. All DACs 
are equipped with $600 \mu \mathrm{m}$ culet diamonds. In all high-pressure experiments, 718 Inconel gaskets with a starting diameter hole in the range of $280-380 \mu \mathrm{m}$ were used. The pressure inside the sample chamber was measured according to the ruby fluorescence method ${ }^{23}$ using an in-house built kit that has a precision of $0.05 \mathrm{GPa}$.

Initially, two approaches were adapted to explore inclusion complex formation in a DAC. The first one consisted of using a hot equimolar solution of guest and host compounds. Such a solution is supersaturated with respect to one of the solutes, as the water solubility of the host and guest compounds are different. The second approach is related to the preliminary screening at ambient conditions: if a 1:1 inclusion complex forms at ambient-pressure conditions, then loading small crystals of the complex into the DAC and subsequently dissolving them in situ is more convenient than loading a hot solution, ensuring a larger quantity of the solute is loaded in the DAC. Water as pressure transmitting medium enables an exploration range from atmospheric up to $c$ a. 1.5 GPa. All explored CD-drug combinations are given below and summarised in Table 5.3.

Table 5.3. Summary of the experimental inclusion complex screening at high-pressure condition.

\begin{tabular}{|c|c|c|}
\hline & in $\alpha$-CD-water & in $\beta$-CD.water \\
\hline $5 \mathrm{FU}$ & $\begin{array}{l}\text { Single crystals obtained in situ by } \\
\text { temperature and pressure cycling }\end{array}$ & $\begin{array}{l}\text { Single crystals obtained in situ by } \\
\text { temperature and pressure cycling }\end{array}$ \\
\hline Amoxicillin & $\begin{array}{c}\text { This combination has not been } \\
\text { investigated }\end{array}$ & $\begin{array}{l}\text { Increased dissolution of amoxicillin } \\
\text { observed as a function of increasing } \\
\text { pressure. No crystallisation observed } \\
\text { once a clear solution is formed. }\end{array}$ \\
\hline Succinic acid & $\begin{array}{c}\text { No crystallisation observed. See } \\
\text { Chapter } 3 \text { for details }\end{array}$ & $\begin{array}{l}\text { Single crystals obtained in situ by } \\
\text { temperature and pressure cycling }\end{array}$ \\
\hline L-ascorbic acid & $\begin{array}{l}\text { This combination has not been } \\
\text { investigated }\end{array}$ & $\begin{array}{l}\text { No crystallisation observed at high } \\
\text { pressure }\end{array}$ \\
\hline
\end{tabular}

\subsubsection{1. $\alpha-\mathrm{CD} \cdot 5 \mathrm{FU}$}

A rectangular-shaped beryllium-DAC was loaded with a hot equimolar solution of $\alpha$-CD and 5FU. Small single crystals crystallised after closing the DAC at 0.24 GPa (Figure 5.2.a). Heating the DAC using a heatgun at $343 \mathrm{~K}$ led to dissolution at the same pressure conditions (Figure 5.2.b). Cooling of the DAC to room temperature promoted the recrystallisation of the material and was associated with a drop in the pressure to $0.19 \mathrm{GPa}$. Increasing the pressure gradually up to 0.84 GPa did not induce further crystallisation or dissolution events (Figure 5.2.c-g). The single crystal in Figure 5.2.g was measured by means of single crystal X-ray diffraction using synchrotron radiation (PETRA synchrotron, Hamburg, beamline P02.2). When pressure was released to ambient, the crystal partially dissolved (Figure 5.2.h). 


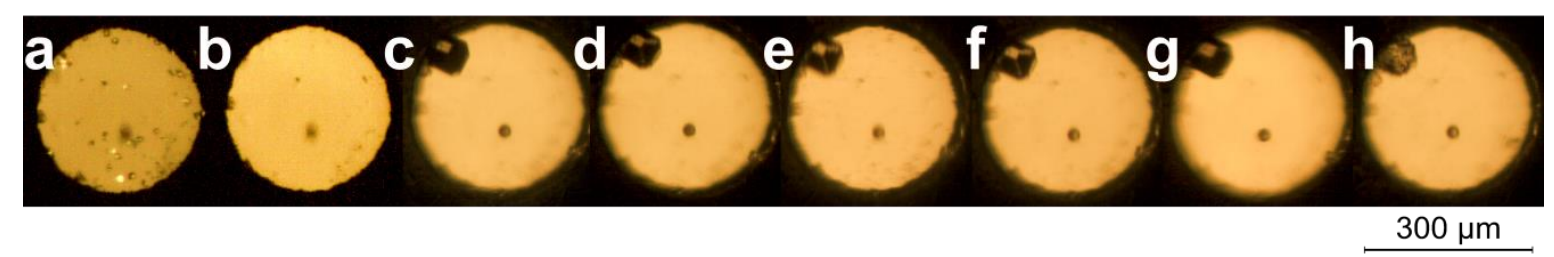

Figure 5.2. Crystallisation attempt of a hot aqueous solution of $\alpha-C D \cdot 5 F U$ in a DAC. a) DAC loaded with hot and saturated solution of $\alpha-\mathrm{CD}$ and 5FU; b) complete dissolution of the crystals at $c a .343 \mathrm{~K}$ and ca. 0.24 GPa; c) a single crystal crystallised after complete cooling of the DAC at $0.19 \mathrm{GPa}$; $-\mathrm{g}$ ) single crystal stable after gradual pressure increase of $0.36,0.45,0.59,0.84 \mathrm{GPa}$, respectively; $\mathrm{h}$ ) single crystal partially dissolved after releasing pressure to ambient.

\subsubsection{2. $\beta$-CD·5FU}

A round-shaped beryllium-DAC was loaded with a hot equimolar solution of $\beta$-CD and 5FU. Sealing the DAC promoted instantaneous crystallisation (Figure 5.3.a). Increasing the pressure inside the DAC to 0.32 (Figure 5.3.b) then 0.43 GPa (Figure 5.3.c-f) induced complete dissolution of the crystals. Recrystallisation was not observed at the same pressure. Increasing pressure to $0.57 \mathrm{GPa}$ did not promote crystallisation. However, after increasing pressure to 0.78 GPa, slow crystallisation of a single crystal occurred (Figure 5.3.g). Single-crystal X-ray diffraction was carried out on the same crystal using synchrotron radiation (PETRA synchrotron, Hamburg, beamline P02.2).

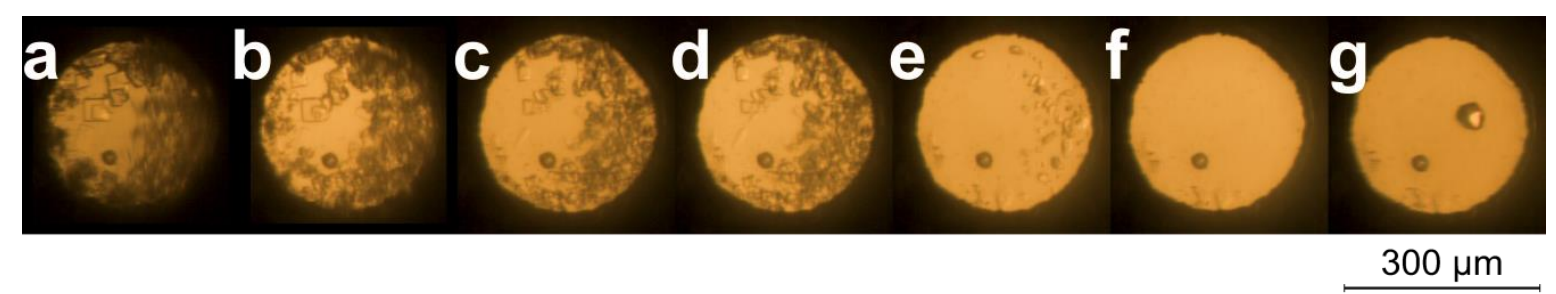

Figure 5.3. Crystallisation attempt of a hot aqueous solution of $\beta$-CD 5 FU in a DAC. a) Crystallisation of the hot equimolar solution of $\beta$-CD and $5 \mathrm{FU}$ at $0.11 \mathrm{GPa} ; \mathrm{b}$ ) dissolution of the crystals after pressure increase to

$0.32 \mathrm{GPa}$; c-f) dissolution is complete after a further pressure increase to $0.43 \mathrm{GPa}$; $\mathrm{g}$ ) a single crystal is obtained after $12 \mathrm{~h}$ at $0.78 \mathrm{GPa}$.

\subsubsection{3. $\beta$-CD·L-ascorbic acid}

A beryllium-free modified Merrill-Bassett DAC was loaded with a hot solution of an equimolar $\beta$-CD and L-ascorbic acid (Figure 5.4.a). After sealing the DAC to a pressure of 0.4 GPa, crystallisation was not observed (Figure 5.4.b). $12 \mathrm{~h}$ of cooling to $283 \mathrm{~K}$ (Figure 5.4.c), or crystallising ice above 1.5 GPa (Figure 5.4.d) and cycling pressure between 1.13 and $0.52 \mathrm{GPa}$ did not promote and crystallisation event (Figure 5.4.e-g). 


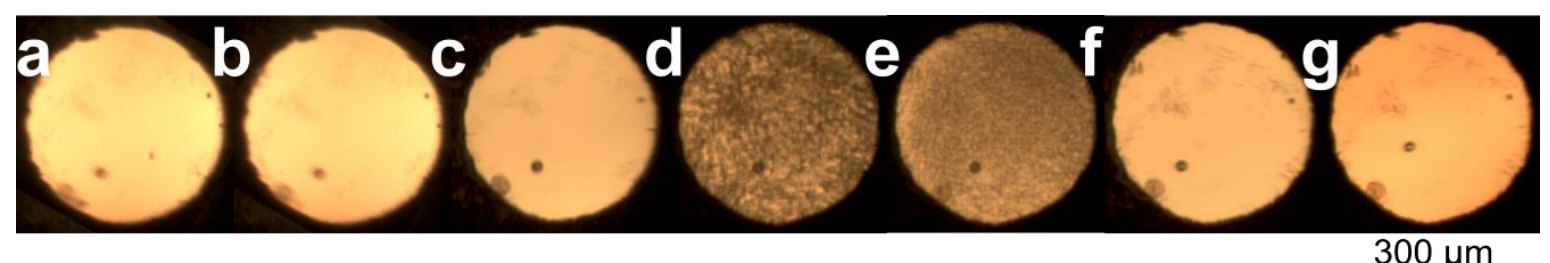

Figure 5.4. High-pressure crystallisation attempt of an equimolar solution of $\beta$-CD and L-ascorbic acid.

a) DAC at 0.4 GPa with hot aqueous solution of $\beta$-CD and L-ascorbic acid; b) no change after $24 \mathrm{~h}$ at the same conditions; c) no change after cooling to 283 for 12 h; d) ice VI formed after pressure increase above 1.6 GPa; e)

final stage of ice after slow growth, $\mathrm{P}=1.13 \mathrm{GPa}$; ) ice melted by releasing pressure, $\mathrm{P}=0.77 \mathrm{GPa}$; ) no crystallisation after $12 \mathrm{~h}$ at $288 \mathrm{~K}$ and $0.52 \mathrm{GPa}$.

\subsubsection{4. $\beta$-CD·succinic acid}

A beryllium-free DAC of the Ahsbahs type was loaded with the known $\beta$-CD.SA complex. ${ }^{24}$ Sealing the DAC and increasing pressure to $0.54 \mathrm{GPa}$ induced a slow dissolution of the complex (Figure 5.5.a-b). A further increase of the pressure inside the DAC to 0.88 GPa provoked a rapid and complete dissolution (Figure 5.5.c-e). Crystallisation occurred after a second pressure increase to 1.0 GPa (Figure 5.5.f-g). The crystals were too small for X-ray diffraction experiments using a laboratory source and were not analysed further.

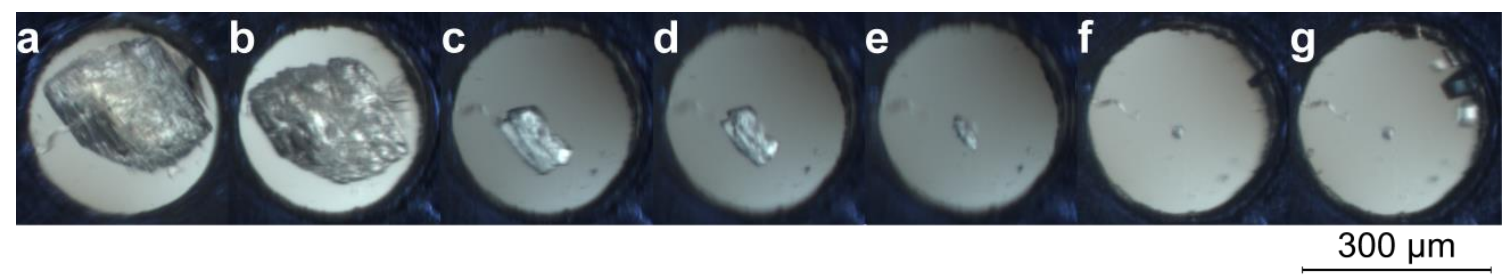

Figure 5.5. Recrystallisation attempts of $\beta$-CD·SA complex at high pressure. a) DAC loaded with a crystal of $\beta$-CD.SA and sealed at $0.2 \mathrm{GPa} ; \mathrm{b}$ ) dissolution of the complex after pressure increase to $0.54 \mathrm{GPa} ; \mathrm{c}-\mathrm{e})$ dissolution enhanced after further pressure increase to $0.88 \mathrm{GPa}$, pictures captured at 2 min interval; f) no recrystallisation at $0.88 \mathrm{GPa}$ after 3 hours, recrystallisation (top right) after pressure increase to $1.0 \mathrm{GPa}$; ) maximum growth of the single crystals after a few hours at the same pressure.

\subsubsection{5. $\beta$-CD·amoxicillin}

Crystallising a hot equimolar solution of $\beta-C D$ and amoxicillin in a DAC was unsuccessful and has been related to the low solubility of amoxicillin. The pressure range of the experiment was between $0.3-0.88$ GPa pressure with $12 \mathrm{~h}$ cooling at $283 \mathrm{~K}$.

A further qualitative experiment was also carried out: two single crystals of $\beta$-CD (Figure 5.6.a right) were loaded together with a single crystal of amoxicillin (Figure 5.6.a left) trihydrate into a DAC. Sealing the DAC and increasing pressure induces the dissolution of $\beta$-CD crystals (Figure 5.6.b-g). Interestingly, in the same pressure range we observed a slow dissolution of amoxicillin, presumably due to complex formation in solution. The dissolution of amoxicillin increases as function of pressure (Figure 5.6.g-j). Cycling pressure and temperature did not lead to 
any crystallisation apart from that of ice VI. Releasing pressure to ambient and opening the DAC led to the formation of an amorphous solid.

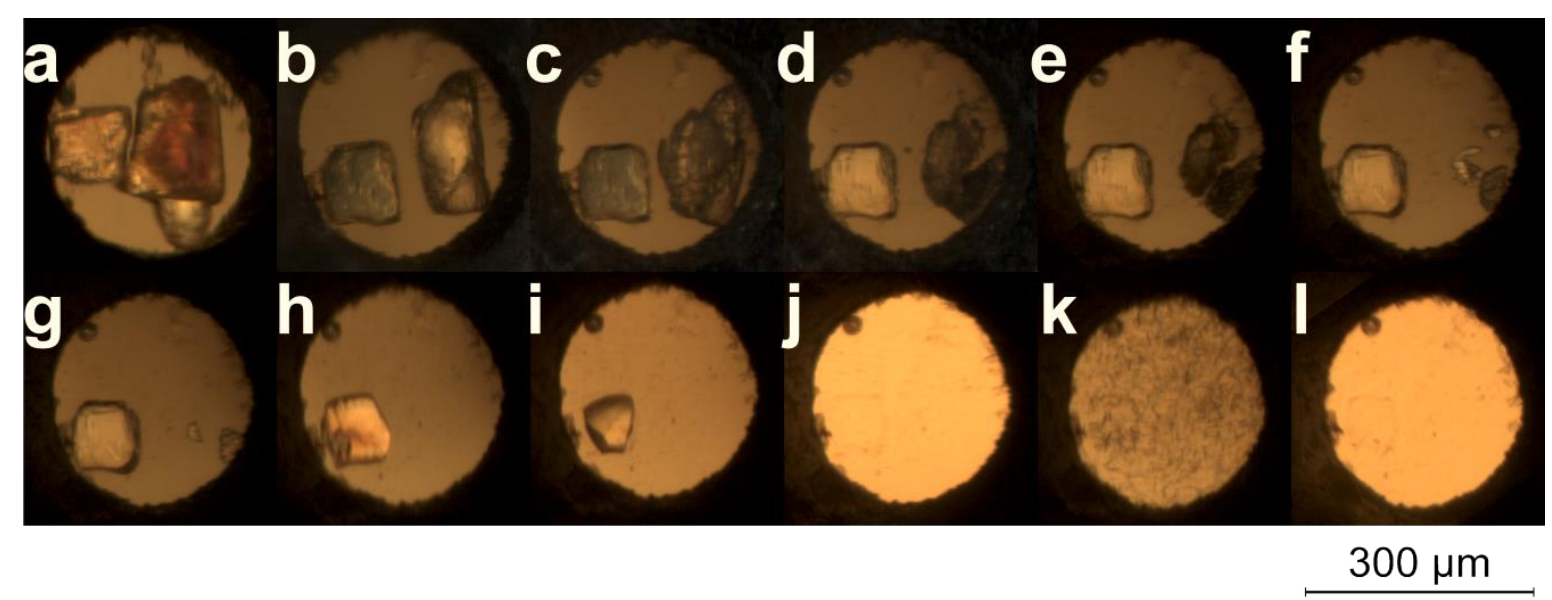

Figure 5.6. Exploring the behaviour of single crystals of amoxicillin and $\beta$-CD at high-pressure. a) a DAC loaded with single crystals of amoxicillin (left) and $\beta-\mathrm{CD}$ (right); b) dissolution of $\beta$-CD after sealing the DAC, $\mathrm{P}=0.23 \mathrm{GPa}$; $\mathrm{c}$ ) slow dissolution of $\beta$-CD crystal at the same pressure; $\mathrm{d}$-g) Faster dissolution of $\beta$-CD crystal after pressure increase to $0.65 \mathrm{GPa}$ and slow dissolution of amoxicillin crystal; $\mathrm{g}$-j) faster dissolution of amoxicillin crystal after further increase in P to $0.98 \mathrm{GPa}$; ) crystallisation of ice at after cooling to $277 \mathrm{~K}$ for 12 $\mathrm{h}$; l) clear solution at $1.06 \mathrm{GPa}$ and ambient-temperature conditions.

A subsequent experiment to test the water solubility of amoxicillin as function of pressure was carried out. Dissolution of amoxicillin trihydrate crystal did not occur in the atmospheric - 1.5 GPa pressure range as evidenced in Figure 5.7, confirming that the increased solubility observed in the previous experiment was due to interaction of the drug with $\mathrm{CD}$ at high pressure.

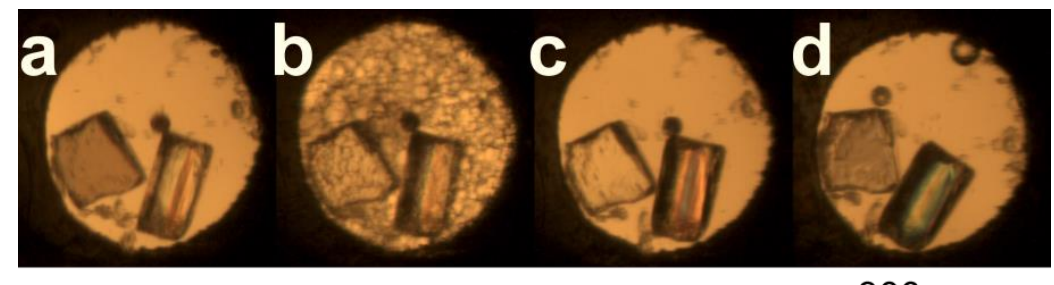

$300 \mu \mathrm{m}$

Figure 5.7. Investigating the dissolution at high pressure of amoxicillin in water. a) Single crystals of amoxicillin trihydrate in water at $0.34 \mathrm{GPa} ; \mathrm{b}$ ) ice surrounding the same crystals after gradual pressure increase to $1.5 \mathrm{GPa}$; ) no dissolution of the crystals by pressure release; d) DAC open, atmospheric pressure.

\subsubsection{High-pressure experiment using a stationary gas compressor}

Following the qualitative observation made on the $\beta$-CD.amoxicillin.water system in a DAC, a larger-scale crystallisation experiment was carried out using a stationary gas compressor. The aim of the experiment was to repeat the experiment performed with the DAC under more controlled conditions and to investigate other crystallisation routes.

The stationary gas compressor enables compressions up to $0.7 \mathrm{GPa}$ with an accuracy of $c a$. $2 \%$. Compression is carried out in two stages and the experiment can be simplified as follows: 1 ) 
the system is filled in with a continuous flow of a gas from a cylinder; 2 ) the gas is pre-compressed in a compressor to at least $0.03 \mathrm{GPa} ; 3$ ) the pre-compressed gas is introduced to the intensifier and is compressed further to a desired value below $0.7 \mathrm{GPa}$; 4 ) the compressed air is introduced gradually to the pressure cell.

Nitrogen gas was used as pressure transmitting medium. The pressure cell was loaded with $7.2 \mathrm{mg}$ of amoxicillin ( $\mathrm{MM}=365.4 \mathrm{~g} / \mathrm{mol}), 22.5 \mathrm{mg}$ of $\beta-\mathrm{CD}(\mathrm{MM}=1135 \mathrm{~g} / \mathrm{mol})$ and $1 \mathrm{ml}$ of demineralised water (Figure 5.8.a). The cell was subsequently connected to the high-pressure system and checked for leakage. The pressure cell was then immersed in a water bath and preheated to $313 \mathrm{~K}$. The experiment was undertaken at the same constant temperature at which nitrogen hydrate does not form at pressures up to $0.5 \mathrm{GPa}$, as calculated using CSMGem program. ${ }^{25}$ After 2.5 hours at a constant pressure of $0.5 \mathrm{GPa}$, the pressure was released gradually and the sample was checked under a polarised optical microscope (Figure 5.8.b). The solution was filtered and the filtrate was place at $277 \mathrm{~K}$ for several days (Figure 5.9).

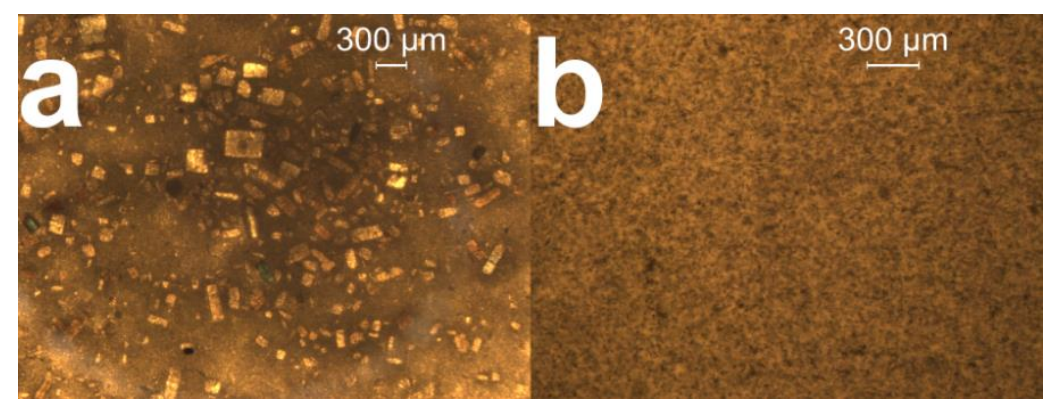

Figure 5.8. $\beta$-CD and amoxicillin solution (a) before and (b) after applying $0.5 \mathrm{GPa}$ pressure.

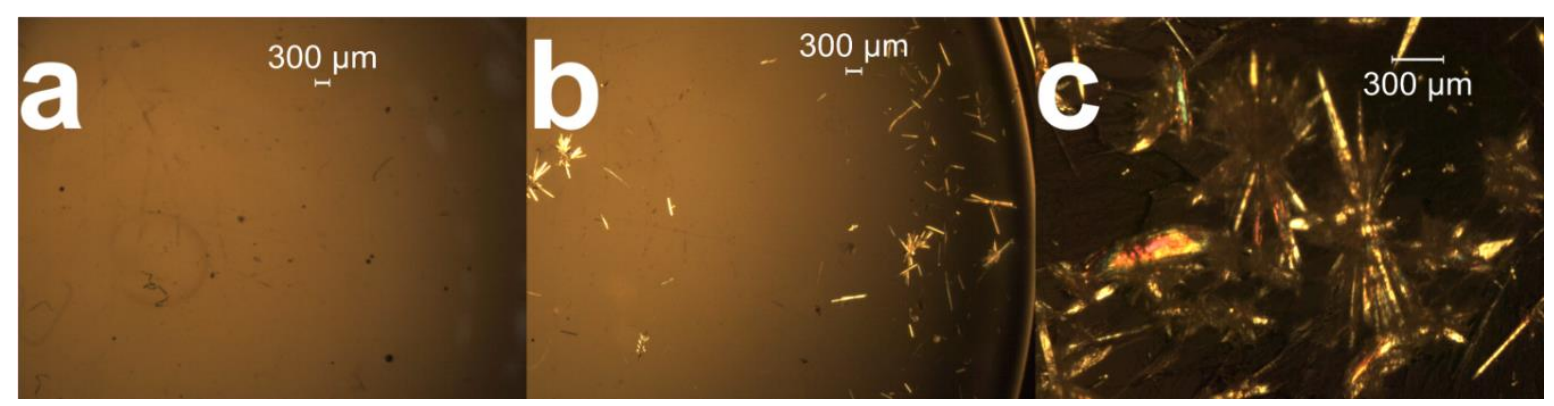

Figure 5.9. Slow evaporation of the filtrate of $\beta$-CD-amoxicillin solution. a) Clear filtrate of $\beta$-CD-amoxicillin solution as seen in Figure 5.8.b; b) crystallisation of needle-like crystals after 4 day of slow evaporation at 277 $\mathrm{K} ; \mathrm{c})$ a second population of crystals is observed after complete evaporation of the solvent.

\subsubsection{X-ray diffraction}

Single-crystal X-ray diffraction studies on the outcome of the crystallisation at ambientpressure conditions were carried out on either 1) a Bruker SMART 6000 Apex II CCD diffractometer, equipped with $\mathrm{Cu} K \alpha$ rotating anode radiation of $\lambda=1.54178 \AA$, or 2) a Bruker AXS SMART Apex II CCD diffractometer equipped with Mo K $\alpha$ sealed-tube radiation of $\lambda=0.71073 \AA$. Both diffractometers are equipped with an Oxford Cryosystems Cobra low-temperature device. 
The diffraction experiments are summarised in Table 5.4. High-pressure data were collected partly on the same Bruker AXS SMART Apex II CCD diffractometer, or using synchrotron radiation at the extreme conditions beamline P02.2 of the PETRA synchrotron ring at DESY (Hamburg, Germany), using a microfocused beam of $\lambda=0.28962 \AA$, and a large Perkin Elmer XRD 1621 detector.

\subsection{Results and discussion}

A screening of inclusion complex formation using $\alpha-, \beta$ - and hp- $\beta$-CD has been carried out at ambient- and high-pressure crystallisation conditions. The primary aim of the study was to investigate the solid-state behaviour of CD·drug.water system at high pressure, which has been previously, to the best of our knowledge, unexplored, and secondly to qualitatively monitor improvements in water solubility ${ }^{26,27}$ of the studied drug compounds.

\subsubsection{Ambient pressure}

A polarised optical microscopy comparison on the crystallisation rates of pure $\alpha-, \beta$ - and $\mathrm{hp}-\beta$-CDs has been carried out revealing the following $26,27: \beta$-CD crystallises from an undersaturated solution within hours and from a supersaturated solution within minutes. $\alpha-\mathrm{CD}$ crystallises within days after very slow evaporation; a faster evaporation rate promotes glass formation; the crystallisation could be accelerated by cooling the solution to $277 \mathrm{~K}$, however, in the case of an undersaturated solution the single crystals rapidly dissolve when temperature reaches ambient. Slow evaporation of hp- $\beta$-CD always yields an amorphous solid. The crystallisation rates of $\alpha-, \beta$ - and hp- $\beta$-CDs are inversely proportional to their solubility values (Table 5.1). All the results and observations of the ambient pressure investigations of inclusion complexes formation are summarised in Table 5.4.

Table 5.4. Summary of the single-crystal X-ray diffraction results on the inclusion complex screening investigated at ambient-pressure conditions.

\begin{tabular}{|c|c|c|c|}
\hline & in $\alpha$-CD.water & in $\beta$-CD.water & in $h p-\beta$-CD water \\
\hline Carbamazepine & l & $\begin{array}{c}\text { recrystallisation of carbamazepine } \\
\text { as a dihydrate (as in refcode } \\
\text { FEFNOT02) }{ }^{28}\end{array}$ & $\begin{array}{c}\text { recrystallisation of carbamazepine } \\
\text { as a dihydrate (as in refcode } \\
\text { FEFNOT02) }{ }^{28}\end{array}$ \\
\hline Aspartame & l & recrystallisation of $\beta$-CD hydrate & / \\
\hline 5-fluorouracil & $\begin{array}{l}\text { 5FU recrystallises in the triclinic } \\
\text { form (as in refcode FURACL) }{ }^{29}\end{array}$ & $\begin{array}{l}\text { suspected formation of } \beta-C D \cdot 5- \\
\text { fluorouracil inclusion complex }\end{array}$ & l \\
\hline Ampicillin & l & l & l \\
\hline Gabapentin & recrystallisation of $\alpha-C D$ hydrate & recrystallisation of $\beta$-CD hydrate & l \\
\hline L-dopa & 1 & $\begin{array}{l}\text { L-dopa recrystallises in the } \\
\text { monoclinic form (as in } \\
\text { LDOPAS03) }\end{array}$ & l \\
\hline Piracetam & l & recrystallisation of $\beta$-CD hydrate & l \\
\hline
\end{tabular}


6-APA, amoxicillin and ampicillin belong to the penicillin's family of antibiotics. It is well known that penicillins are unstable in aqueous solution. This effect was already known to their discoverer, Alexander Fleming. ${ }^{31}$ Water, acting as a nucleophile, attacks the strained $\beta$-lactam ring and opens it. This effect is exacerbated when heating is applied. ${ }^{32}$ We observed in our experiments that the clear transparent solution of $\alpha-, \beta$ - and hp- $\beta$-CDs with penicillins turn first yellow and ultimately to dark orange after a few days. The change in colour can be explained by the degradation of the penicillins. The same behaviour was noticed for L-dopa: the CD-based solutions of L-dopa turned from clear transparent to light then dark brown, as function of time. The degradation of L-dopa in aqueous solution is known. ${ }^{33}$ Studies reported in the literature indicate that in solution amoxicillin forms 1:1 complexes with $\beta$ - and $\gamma$-cyclodextrin, whereas no complex is formed with $\alpha$-CD. When complexed with $\beta-\mathrm{CD}$ and at conditions of $\mathrm{pH} 1.2$ and $37^{\circ} \mathrm{C}$, the degradation rate of the drug is slower. ${ }^{34}$

The solubility of carbamazepine has been reported to increase in the presence of $\beta$-CD. ${ }^{35}$ The formation of a 1:1 complex in the solid state has also been reported but such a complex has not been structurally characterised. ${ }^{36}$ We observed that on cooling an equimolar solution heated to 353 $\mathrm{K}$, carbamazepine recrystallises faster than CD. Single crystals of the recrystallisation outcome with $\beta$ - and hp- $\beta$-CD were tested and gave similar unit cell constants to those of carbamazepine $\cdot 2\left(\mathrm{H}_{2} \mathrm{O}\right)$, CSD refcode FEFNOT02..$^{28}$ Aspartame has been reported to form inclusion complex in solution with different $\mathrm{CD}$ s and preferentially with $\beta$-CD derivatives. ${ }^{37}$ Aspartame in CD-based solutions formed aggregates; following heating and cooling cycles, only recrystallisation of the known form of $\beta$-CD hydrate was detected.

The observed increased solubility of 5FU in presence of $\alpha$-CD was firstly thought to be associated with inclusion complex formation, although this observation has not been checked experimentally using analytical techniques. Slow evaporation of a clear equimolar aqueous solution yields the crystallisation of bulky crystals of 5FU in the triclinic form ${ }^{29}$ whilst $\alpha$-CD remains in solution. Water solubility of $5 \mathrm{FU}$ increased in $\beta-\mathrm{CD}$ solution as well and recrystallisation occurred after slow evaporation. X-ray diffraction on the resulting single crystals of $\beta$-CD.5FU at $100 \mathrm{~K}$ indicated that the unit-cell parameters are those of the known monoclinic $P 21$ form $^{38}$ of $\beta$-CD hydrate. $5 \mathrm{FU}$ is a small molecule that could potentially fit in $\beta$-CD cavities. Structure solution and refinement show considerable electron density within the $\beta$-CD core, which could translate to the presence of at least one highly disordered 5FU molecule distributed over at least three positions. Although fully restrained 5FU models were used, it was not possible to refine the model satisfactorily. In order to prove the presence of $5 \mathrm{FU}$ inside $\beta$-CD cavity, $\beta$-CD hydrate structures containing a variable amount of water (CSD refcodes BCDEXD03, BCDEXD04, BCDEXD05 and BCDEXD10)38,39 were taken as models for refinement. While water molecules fit 
partly the electron density inside the cavity, substantial residual density is still present and the structure needs further modelling. 5FU has been previously reported to form an inclusion compound with carboxymethyl- $\beta-\mathrm{CD} ;{ }^{40}$ Collection of better quality X-ray diffraction data may be helpful and complementary techniques, such as Raman spectroscopy, should also be considered to confirm the existence of 5FU molecules in the crystal structure.

Data collected on single crystals resulting from the solutions containing $\beta$-CD together with piracetam and gabapentin gave unit-cell parameters corresponding to the monoclinic $P 21 \beta$ CD hydrate. ${ }^{38}$ Refinements based on this structure give very good R-factors (5.68 \% and $5.25 \%$, respectively) and no suspicious residual electron density. Hence, it can be concluded $\beta$-CD does not form inclusion complex with either piracetam or gabapentin under the tested ambient-pressure conditions. Complex formation of $\beta-\mathrm{CD}$ with gabapentin in solution has been previously reported. ${ }^{41}$

In the case of the crystals resulting from $\beta$-CD·L-ascorbic acid mixtures, refinement of the $\beta$-CD hydrate model was not satisfactory. The difference Fourier maps reveals high unassigned electron density peaks inside $\beta$-CD cavity; assigning further water molecules to these peaks did not solve the problem as additional peaks appear; it is not unlikely that L-ascorbic acid is extensively disordered inside $\beta$-CD cavity. A Raman spectroscopy study should be carried out in order to confirm inclusion complex formation. However, L-ascorbic acid has been previously reported to form inclusion complexes in solution at ambient ${ }^{42}$ and high hydrostatic pressure of $0.3 \mathrm{GPa} .{ }^{43}$

Table 5.5. Crystal data of all measured single crystals following the inclusion complex screening at ambient conditions.

\begin{tabular}{|c|c|c|c|c|c|c|c|c|}
\hline $\begin{array}{c}\text { Solution } \\
\text { used for } \\
\text { crystallisation }\end{array}$ & $\alpha-\mathrm{CD} \cdot 5 \mathrm{FU}$ & $\begin{array}{c}\alpha-C D \\
\text { gabapentin }\end{array}$ & $\begin{array}{c}\beta-\mathrm{CD} \\
\text { carbamazepine }\end{array}$ & $\begin{array}{c}\beta-C D \cdot \\
\text { aspartame }\end{array}$ & $\beta-\mathrm{CD} \cdot 5 \mathrm{FU}$ & $\begin{array}{c}\beta \text {-CD. } \\
\text { gabapentin }\end{array}$ & $\begin{array}{c}\beta-\mathrm{CD} \cdot \mathrm{L}- \\
\text { ascorbic acid }\end{array}$ & $\begin{array}{c}\text { hp- } \beta \text {-CD. } \\
\text { carbamazepine }\end{array}$ \\
\hline Space group & $P-1$ & $P 212121$ & $P 2_{1} / \mathrm{c}$ & $P 2{ }_{1}$ & $P 2_{1}$ & $P 2_{1}$ & $P 2_{1}$ & $P 21 / \mathrm{c}$ \\
\hline$a / \AA ̊$ & $8.832(5)$ & $9.5250(3)$ & 10.2773 & 15.01 & 14.9071(19) & $15.1421(5)$ & $14.9727(8)$ & $10.01(5)$ \\
\hline b/Å & $9.276(5)$ & $14.8680(5)$ & 28.9900 & 10.23 & $10.1255(13)$ & $10.2078(4)$ & $10.1815(6)$ & $28.21(13)$ \\
\hline$c / \AA ̊$ & $12.724(6)$ & $34.0068(11)$ & 4.9800 & 20.83 & $20.848(3)$ & $20.9376(7)$ & $21.2696(14)$ & $4.85(2)$ \\
\hline$\alpha /^{\circ}$ & $81.375(8)$ & 90 & 90 & 90 & 90 & 90 & 90 & 90 \\
\hline$\beta /^{\circ}$ & $80.668(9)$ & 90 & 103.9888 & 108.99 & $108.949(5)$ & $110.876(2)$ & $112.316(2)$ & $103.99(3)$ \\
\hline$\gamma /{ }^{\circ}$ & $89.248(13)$ & 90 & 90 & 90 & 90 & 90 & 90 & 90 \\
\hline$V / \AA^{3}$ & 1017 & 4815.96 & 1439.727 & 3025 & 2976.3 & 3023.82 & 2999.59 & 1331.147 \\
\hline$Z^{\prime}$ & 4 & 1 & 1 & 1 & 1 & 1 & 1 & 1 \\
\hline$T / K$ & $298(2)$ & $90(2)$ & $298(2)$ & $100(2)$ & $100(2)$ & $90(2)$ & $100(2)$ & $298(2)$ \\
\hline $\begin{array}{c}\text { Structure } \\
\text { solution and } \\
\text { refinement }\end{array}$ & $5 \mathrm{FU}$ & $\begin{array}{c}\alpha-\mathrm{CD} \\
\text { hydrate }\end{array}$ & Carbamazepine & $\begin{array}{c}\beta-C D \\
\text { hydrate }\end{array}$ & $\begin{array}{c}\text { Complex } \\
\text { (disordered } \\
\text { guest) }\end{array}$ & $\begin{array}{c}\beta-C D \\
\text { hydrate }\end{array}$ & $\begin{array}{c}\text { Complex } \\
\text { (disordered } \\
\text { guest) }\end{array}$ & Carbamazepine \\
\hline
\end{tabular}




\subsubsection{High pressure}

The pressure range explored in the high-pressure screening varies from atmospheric to $c a$. 1.2 GPa, the upper limit being limited by the use of water as pressure transmitting medium. The use of hot aqueous solutions of equimolar host and guest ratio for DAC loading allows to better control the concentration of the solutes in the DAC but usually leads the formation of small crystal. One of the limitations associated with this type of loading is the preparation of such solutions when poorly soluble drugs are used. Drugs with low solubility, and for which crystals of an inclusion complex with CDs are known to not form at ambient conditions are particularly challenging. Heating the sample can help with increasing solubility but is also of limited use: for instance the instability of the $\beta$-lactam group and L-dopa are known to increase when temperature is increased. If a complex can be obtained at ambient conditions, loading of single crystals of the complex in the DAC enables higher concentrations to be reached in situ by dissolving the crystal as function of applying pressure. However, this loading route gives very little control over the starting concentration of the solutes. This is a relatively small drawback that enables to explore the effects of pressure for driving CD complex formation for a wider range of compounds.

Table 5.6. Summary of the single-crystal X-ray diffraction results on the inclusion complex screening investigated at high- pressure conditions.

\begin{tabular}{ccc}
\hline & in $\alpha$-CD $\cdot$ water & in $\beta$-CD·water \\
\hline 5FU & $\begin{array}{c}5 \mathrm{FU} \text { recrystallises in the triclinic form } \\
(\text { as in refcode FURACL })^{29} \\
\text { This combination has not been } \\
\text { investigated }\end{array}$ & $\begin{array}{c}5 \mathrm{FU} \text { recrystallises in the triclinic form } \\
(\text { as in refcode FURACL })^{29} \\
\text { No crystallisation observed }\end{array}$ \\
Succinic acid & $\begin{array}{c}\text { No crystallisation observed. See } \\
\text { Chapter } 3 \text { for details }\end{array}$ & $\begin{array}{c}\text { Crystals too small for analysis using } \\
\text { laboratory equipment }\end{array}$ \\
L-ascorbic acid & $\begin{array}{c}\text { This combination has not been } \\
\text { investigated }\end{array}$ & $\begin{array}{c}\text { No crystallisation observed at high } \\
\text { pressure }\end{array}$ \\
\hline
\end{tabular}

\subsubsection{1. $\alpha-\mathrm{CD} \cdot 5 \mathrm{FU}$}

In the high-pressure experiment, an increase of the water solubility of 5FU in presence of $\alpha$-CD was observed, which could be related to $\alpha$-CD·5FU inclusion complex formation in solution; however, applying pressure to the hot solution induce the recrystallisation of $5 \mathrm{FU}$ in the triclinic form, ${ }^{29}$ confirmed by single-crystal X-ray diffraction. Further crystallisation and phase transitions have not been observed when increasing pressure up to $0.84 \mathrm{GPa}$.

\subsubsection{2. $\beta$-CD.5FU}

The outcome of the crystallisation experiment described in section 5.3.3.2, which occurs at pressures higher than $0.6 \mathrm{GPa}$, is a crystal of the guest compound in the triclinic form I. ${ }^{29}$ This was confirmed by in situ single crystal diffraction at $0.78 \mathrm{GPa}$. There was no evidence of the crystallisation of $\beta$-CD or $\beta$-CD·5FU complex at high pressure. 


\subsubsection{3. $\beta$-CD·L-ascorbic acid}

The high-pressure experiment shows no evidence of crystallisation in the explored pressure range. Szente and Szejtli ascribed the amorphousness of $h p-\beta-C D$ to the random hydroxypropylation on the $(3 \times 7) \beta$-CD hydroxy groups, giving a combination of more than two million different molecules in the pool. ${ }^{44}$ It is important to note that the authors reported a high number, which does not discard distinguishable combinations. Nevertheless, the number of species in the solution stays very high even with discarding distinguishable combinations. A subsequent experiment on a concentrated aqueous solution of hp- $\beta$-CD alone was carried out, and also in this case no crystallisation was observed in the full pressure range of liquid water.

\subsubsection{4. $\beta$-CD·succinic acid}

In the solid state, $\beta$-CD is known to form an inclusion complex with succinic acid at ambient-pressure conditions. ${ }^{24}$ By loading a single crystal of the complex into a DAC and increasing pressure dissolution was observed (Figure 5.5). Although the amount of starting material in the DAC was considerable, the crystals formed at high pressure were small and unsuitable for an in house X-ray diffraction study. In subsequent experiments on the same system, we observed recrystallisation at high pressure similar to that reported in Figure 5.5.g but of two clearly distinct populations of small crystals. This could in theory correspond to crystallisation of the solutes as distinct compounds, as observed in the experiment with 5FU and with paracetamol (for the latter see Chapter 4 section 4.4.4.2). Raman spectroscopic investigations should be carried out to confirm this hypothesis.

\subsubsection{5. $\beta$-CD·amoxicillin}

As explained earlier, it is difficult to assess the exact amount of material loaded into the DAC when single crystals of inclusion complexed are loaded; hence this study is rather qualitative. Polarised optical microscopy shows that when single crystals of amoxicillin and of $\beta$-CD are loaded into a DAC, and by using water as pressure transmitting medium, applying pressure results in the expected observation of $\beta-\mathrm{CD}$ dissolving as function of increasing pressure. Interestingly, the dissolution of $\beta-\mathrm{CD}$ induces the dissolution of amoxicillin, which in the case of the performed experiment completely dissolved at $0.98 \mathrm{GPa}$. Further pressure and temperature cycles did not promote any crystallisation except that of ice VI above 1.5 GPa. A subsequent experiment showed that a single crystal of amoxicillin does not undergo dissolution in water when compressed; this confirms that the dissolution of amoxicillin observed in the earlier experiment is caused by the presence and especially the dissolution of $\beta-\mathrm{CD}$ and gives a strong indication that a complex is formed in solution. In the experiment carried out using a stationary gas compressor, $\beta$-CD and amoxicillin were used in equimolar quantities. The starting solution was not heat treated and contained crystals of both the host and guest compounds. After applying pressure (0.5 GPa during 
$2.5 \mathrm{~h}$ ) and releasing it back to ambient, crystals of $\beta$-CD were observed to have apparently dissolved completely whereas some amoxicillin crystals remained in the solution. An X-ray diffraction experiment would have been performed to confirm this but would not be technically straightforward to perform in the laboratory. In a DAC, we could observe that both crystal dissolve when pressure is applied and that an amorphous material is obtained when pressure is released to ambient. Further experiments would be required to establish whether the pressure and concentration regimes used allow to form complete dissolution of amoxicillin at high pressure. Our experience with working with $\beta$-CD indicates that concentrations in excess of $0.2 \mathrm{M}(c a .227 \mathrm{~g} / \mathrm{L})$ can be reached in situ in the DAC by simple compression of $\beta$-CD at room temperature, indicating that $\beta$-CD must have completely dissolved. Filtering the amoxicillin crystals and allowing a slow evaporation of the filtrate yielded crystallisation after four days at $277 \mathrm{~K}$. The needle-shaped crystals were suspected to be those of amoxicillin trihydrate (Figure 5.9.b), and this was subsequently confirmed by single-crystal X-ray diffraction. Further evaporation of the solvent induces crystallisation of block-like crystals (Figure 5.9.c), identified as those of $\beta$-CD hydrate by diffraction.

The combined DAC and stationary gas compressor experiments, demonstrate that it is very likely that $\beta$-CD forms inclusion complex in solution with amoxicillin at high-pressure conditions. The complex is stable in solution but does not crystallise in the explored pressure and temperature ranges. On pressure release amoxicillin trihydrate or amorphous material are obtained. It has been shown that lyophilisation is a viable way to retain complexes in the solid state when these are known to form in solution..$^{45}$ Lyophilisation is a common process used in the pharmaceutical industry as a viable and useful means for safe storage of labile drug products. These observations provide more scope to perform further studies on this and other penicillins. Inclusion complex formation in solution should be confirmed by other experimental techniques such as NMR spectroscopy or UV-Vis spectroscopy.

\subsection{Conclusions}

A small-scale screening of inclusion complex formation at ambient- and high-pressure conditions has been carried out for $\alpha-, \beta$ - and hydroxypropyl- $\beta$-CDs. The purpose of this screen was to acquire working, practical knowledge of handling CD·drug systems, providing the first set of experimental observations at high pressure to be used as a stepping stone for future and more targeted investigations in our group and by others.

At ambient-pressure conditions, two new inclusion complexes were very likely obtained for $\beta$-CD.5FU and $\beta$-CD.L-ascorbic acid, as characterised by means of polarised optical microscopy and single-crystal X-ray diffraction, the latter showing possible extensive disorder of the guest 
molecule inside $\beta$-CD cavities. In the case of $\beta$-CD.piracetam mixtures and $\beta$-CD.gabapentin, refinement of the structural model of $\beta$-CD hydrate was satisfactory; however, even in this case the presence of an extensively disordered guest cannot be ruled out without access to complementary analytical techniques, e.g. Raman spectroscopy, which could be used to confirm or discard inclusion complex formation on one hand, and on the other hand, if appropriate, the molar host guest ratios. The isolation and characterisation of a new complex of $\alpha-\mathrm{CD}$ with succinic acid is reported in full in Chapter 3.

High-pressure investigations on host-guest inclusion complex formation were experimentally very demanding to execute; in particular, the low solubility of some of the drugs used contributed in making the experiments challenging. Because of the size of the drug molecules considered, most experiments were conducted on $\beta$-CD. As far as the current data analysis can tell, the experiments did not lead to the crystallisation of an inclusion complex; for the $\alpha$ - and $\beta$-CD.5FU systems crystallisation of one or both solute components as distinct entities was observed. In the case of $\beta$-CD-succinic acid mixtures further experiments will need to be performed to characterise the crystalline material obtained by in situ crystallisation.

CDs crystals undergo dissolution in aqueous solution at high pressure, regardless of whether guest molecules are present or absent in the cavities. The mechanism for pressure-induced dissolution is currently not fully understood and is the subject of ongoing investigations in our group. We succeeded in exploiting this dissolution trend, which was thought to be an inconvenience at first, and redesign our experiments to explore qualitatively the solubility of drug molecules at high pressure in a $\beta$-CD-based solution. This trend could in principle be of practical application for the dissolution of poorly-water soluble drug molecules. The first qualitative result, demonstrated for amoxicillin, is very promising and suggests that complexation with $\beta-\mathrm{CD}$ is very likely to occur in solution. Such a result would be pertinent in pharmaceutical applications, with $\beta$ CD serving a double role: protection of labile drugs through inclusion complex formation, and controlled release of the drug formulated as a lyophilised inclusion complex.

Further studies related to this subject area, in particular the study of the extent of complexation in solution at high pressure, should also make use of spectroscopic and calorimetric high-pressure techniques to supply quantitative experimental evidence of complexation. These techniques are not currently widespread or widely available and may require substantial investment of resources for custom development. High-pressure studies should also be complemented by the characterisation of CD·drug systems at ambient pressure by performing solubility, spectroscopic and chromatographic studies. 


\subsection{References}

(1) Oswald, I. D. H.; Chataigner, I.; Elphick, S.; Fabbiani, F. P. A.; Lennie, A. R.; Maddaluno, J.; Marshall, W. G.; Prior, T. J.; Pulham, C. R.; Smith, R. I. Putting Pressure on Elusive Polymorphs and Solvates. CrystEngComm 2009, 11 (2), 359.

(2) Fabbiani, F. P. A.; Allan, D. R.; David, W. I. F.; Davidson, A. J.; Lennie, A. R.; Parsons, S.; Pulham, C. R.; Warren, J. E. High-Pressure Studies of Pharmaceuticals: An Exploration of the Behavior of Piracetam. Cryst. Growth Des. 2007, 7 (6), 1115-1124.

(3) Saouane, S.; Norman, S. E.; Hardacre, C.; Fabbiani, F. P. A. Pinning down the Solid-State Polymorphism of the Ionic Liquid [bmim][PF6]. Chem. Sci. 2013, 4 (3), 1270-1280.

(4) Boldyreva, E. V. High-Pressure Diffraction Studies of Molecular Organic Solids. A Personal View. Acta Crystallogr. A. 2008, 64 (Pt 1), 218-231.

(5) Boldyreva, E. V. Multicomponent Organic Crystals at High Pressure. Zeitschrift für Krist. Cryst. Mater. 2014, 229, 236-245.

(6) Das, S.; Das, A. K.; Murphy, R. A.; Worawongvasu, R. Aspartame and Dental Caries in the Rat. Pediatr. Dent. 2000, 13 (4), 217-220.

(7) Taylor, C. P.; Gee, N. S.; Su, T.-Z.; Kocsis, J. D.; Welty, D. F.; Brown, J. P.; Dooley, D. J.; Boden, P.; Singh, L. A Summary of Mechanistic Hypotheses of Gabapentin Pharmacology. Epilepsy Res. 1998, 29 (3), 233-249.

(8) Huot, P.; Johnston, T. H.; Koprich, J. B.; Fox, S. H.; Brotchie, J. M. The Pharmacology of LDOPA-Induced Dyskinesia in Parkinson's Disease. Pharmacol. Rev. 2013, 65 (1), 171-222.

(9) Winblad, B. Piracetam: A Review of Pharmacological Properties and Clinical Uses. CNS Drug Rev. 2005, 11 (2), 169-182.

(10) Longley, D. B.; Harkin, D. P.; Johnston, P. G. 5-Fluorouracil: Mechanisms of Action and Clinical Strategies. Nat. Rev. Cancer 2003, 3 (5), 330-338.

(11) Yen, G.-C.; Duh, P.-D.; Tsai, H.-L. Antioxidant and pro-Oxidant Properties of Ascorbic Acid and Gallic Acid. Food Chem. 2002, 79 (3), 307-313.

(12) Miller, E. L. The Penicillins: A Review and Update. J. Midwifery Women's Heal. 2002, 47 (6), $426-434$.

(13) Ferreira, J. S.; Straathof, A. J. J.; Li, X.; Ottens, M.; Franco, T. T.; van der Wielen, L. A. M. Solution Crystallization Kinetics of 6-Aminopenicillanic Acid. Ind. Eng. Chem. Res. 2006, 45 (20), 6740-6744.

(14) French, D.; Levine, M. L.; Pazur, J. H.; Norberg, E. Studies on the Schardinger Dextrins; the Preparation and Solubility Characteristics of Alpha, Beta and Gamma Dextrins. J. Am. Chem. Soc. 1949, 71 (1), 353-356.

(15) Sigma-Aldrich. (2-Hydroxypropyl)- $\beta$-Cyclodextrin, MSDS No. Aldrich-778966; Steinheim, Germany, 2012.

(16) Sigma-Aldrich. 6-Aminopenicillanic Acid, MSDS No. Aldrich-A70909; Steinheim, Germany, 2013.

(17) Law, V.; Knox, C.; Djoumbou, Y.; Jewison, T.; Guo, A. C.; Liu, Y.; Maciejewski, A.; Arndt, D.; Wilson, M.; Neveu, V.; et al. DrugBank 4.0: shedding new light on drug metabolism.

(18) Crosby, G. A.; Furia, T. E. New Sweeteners. In CRC Handbook of Food Additives; Furia, T. E., Ed.; CRC Press: Boca Raton, Florida, 1980; p 195.

(19) Santa-Cruz-Biotechnology-Inc. Piracetam, MSDS No. SC-204196; Heidelberg, Germany, 2014.

(20) Merrill, L.; Bassett, W. A. Miniature Diamond Anvil Pressure Cell for Single Crystal X-Ray 
Diffraction Studies. Rev. Sci. Instrum. 1974, 45, 290-294.

(21) Moggach, S. A.; Allan, D. R.; Parsons, S.; Warren, J. E. Incorporation of a New Design of Backing Seat and Anvil in a Merrill-Bassett Diamond Anvil Cell. J. Appl. Crystallogr. 2008, 41 (2), 249-251.

(22) Ahsbahs, H. New Pressure Cell for Single-Crystal X-Ray Investigations on Diffractometers with Area Detectors. Zeitschrift für Krist. 2004, 219 (6-2004), 305-308.

(23) Piermarini, G. J.; Block, S.; Barnett, J. D.; Forman, R. A. Calibration of the Pressure Dependence of the R1 Ruby Fluorescence Line to 195 Kbar. J. Appl. Phys. 1975, 46 (6), 27742780 .

(24) Lisnyak, Y. V.; Martynov, A. V.; Baumer, V. N.; Shishkin, O. V.; Gubskaya, A. V. Crystal and Molecular Structure of $\beta$-Cyclodextrin Inclusion Complex with Succinic Acid. J. Incl. Phenom. Macrocycl. Chem. 2007, 58, 367-375.

(25) Sloan Jr., E. D.; Koh, C. A. Clathrate Hydrates of Natural Gases, 3rd ed.; Sloan Jr., E. D., Koh, C., Eds.; CRC Press: Boca Raton, 2007.

(26) Loftsson, T.; Duchêne, D. Cyclodextrins and Their Pharmaceutical Applications. Int. J. Pharm. 2007, 329 (1-2), 1-11.

(27) Davis, M. E.; Brewster, M. E. Cyclodextrin-Based Pharmaceutics: Past, Present and Future. Nat. Rev. Drug Discov. 2004, 3 (12), 1023-1035.

(28) Harris, R. K.; Ghi, P. Y.; Puschmann, H.; Apperley, D. C.; Griesser, U. J.; Hammond, R. B.; Ma, C.; Roberts, K. J.; Pearce, G. J.; Yates, J. R.; et al. Structural Studies of the Polymorphs of Carbamazepine, Its Dihydrate, and Two Solvates. Org. Process Res. Dev. 2005, 9 (6), 902-910.

(29) Fallon, L. The Crystal and Molecular Structure of 5-Fluorouracil. Acta Crystallogr. Sect. B 1973, 29, 2549.

(30) Howard, S. T.; Hursthouse, M. B.; Lehmann, C. W.; Poyner, E. A. Experimental and Theoretical Determination of Electronic Properties in L-Dopa. Acta Crystallogr. Sect. B Struct. Sci. 1995, 51 (3), 328-337.

(31) Fleming, A. On the Antibacterial Action of Cultures of a Penicillium, with Special Reference to Their Use in the Isolation of B.influenzae. Br. J. Exp. Pathol. 1929, 10 (I), 226-236.

(32) Deshpande, A. D.; Baheti, K. G.; Chatterjee, N. R. Degradation of $\beta$-Lactam Antibiotics. Curr. Sci. 2004, 87 (12), 1684-1695.

(33) Pappert, E. J.; Buhrfiend, C.; Lipton, J. W.; Carvey, P. M.; Stebbins, G. T.; Goetz, C. G. Levodopa Stability in Solution: Time Course, Environmental Effects, and Practical Recommendations for Clinical Use. Mov. Disord. 1996, 11 (1), 24-26.

(34) Hidaka, S.; Tokumura, T.; Tomono, K.; Suzuki, T.; Ueda, H.; Nagai, T.; Nagaoka, M.; Nakane, R.; Machida, Y. Effect of Beta-Cyclodextrin on the Degradation Rate of Amoxicillin in Acidic Solution. Yakugaku Zasshi 2010, 130 (6), 889-893.

(35) Koester, L. S.; Bertuol, J. B.; Groch, K. R.; Xavier, C. R.; Moellerke, R.; Mayorga, P.; Dalla Costa, T.; Bassani, V. L. Bioavailability of Carbamazepine:beta-Cyclodextrin Complex in Beagle Dogs from Hydroxypropylmethylcellulose Matrix Tablets. Eur. J. Pharm. Sci. 2004, 22 (2-3), 201-207.

(36) Suresh, S.; Shivakumar, H. N.; Kumar, G. K. Effect of $\beta$ Cyclodextrin Complexation on the Solubility and Dissolution Rate of Carbamazepine from Tablets. Indian J. Pharm. Sci. 2006, 68 (3), 301-307.

(37) Sohajda, T.; Béni, S.; Varga, E.; Iványi, R.; Rácz, Á.; Szente, L.; Noszál, B. Characterization of Aspartame-Cyclodextrin Complexation. J. Pharm. Biomed. Anal. 2009, 50 (5), 737-745. 
(38) Steiner, T.; Koellner, G. Crystalline Beta-Cyclodextrin Hydrate at Various Humidities - Fast, Continuous, and Reversible Dehydration Studied by X-Ray-Diffraction. J. Am. Chem. Soc. 1994, 116 (12), 5122-5128.

(39) Lindner, K.; Saenger, W. Crystal and Molecular Structure of Cyclohepta-Amylose Dodecahydrate. Carbohydr. Res. 1982, 99 (2), 103-115.

(40) Jin, L.; Liu, Q.; Sun, Z.; Ni, X.; Wei, M. Preparation of 5-Fluorouracil / -Cyclodextrin Complex Intercalated in Layered Double Hydroxide and the Controlled Drug Release Properties. Ind. Eng. Chem. Res. 2010, 49 (22), 11176-11181.

(41) Kearney, A. S.; Mehta, S. C.; Radebaugh, G. W. The Effect of Cyclodextrins on the Rate of Intramolecular Lactamization of Gabapentin in Aqueous Solution. Int. J. Pharm. 1992, 78 (1), 25-34.

(42) Ramírez-Silva, M. T.; Palomar-Pardavé, M.; Corona-Avendaño, S.; Romero-Romo, M.; Alarcón-Angeles, G. Guest-Host Complex Formed between Ascorbic Acid and $\beta-$ Cyclodextrin Immobilized on the Surface of an Electrode. Molecules 2014, 19 (5), 5952-5964.

(43) Hu, X.; Wei, B.; Li, H.; Wu, C.; Bai, Y.; Xu, X.; Jin, Z.; Tian, Y. Preparation of the $\beta$ Cyclodextrin-Vitamin $C(\beta-C D-V c)$ Inclusion Complex under High Hydrostatic Pressure (HHP). Carbohydr. Polym. 2012, 90 (2), 1193-1196.

(44) Szente, L.; Szejtli, J. Highly Soluble Cyclodextrin Derivatives: Chemistry, Properties, and Trends in Development. Adv. Drug Deliv. Rev. 1999, 36 (1), 17-28.

(45) Wang, Z.; Deng, Y.; Sun, S.; Zhang, X. Preparation of Hydrophobic Drugs Cyclodextrin Complex by Lyophilization Monophase Solution. Drug Dev. Ind. Pharm. 2006, 32 (1), 73-83. 


\section{PART II.}

\section{Exploring the Solid-State Behaviour of Imidazolium-Based Ionic Liquids}




\section{Chapter 6. Introduction to Part II}

\subsection{Introduction}

Ionic liquids (ILs) are "designer solvents"1 with a melting point below the boiling point of water. They are formed by countless combination of large, usually asymmetric, weakly interacting organic and inorganic cations and anions. ${ }^{2}$ The IL structure-activity relationship can be tuned by selecting a suitable set of ions, therefore generating specific physical and chemical properties and making ILs ideal in terms of technical applicability. The classification of ILs is commonly based on the cation's family. The following is a non-exhaustive list of the most used ILs combinations:
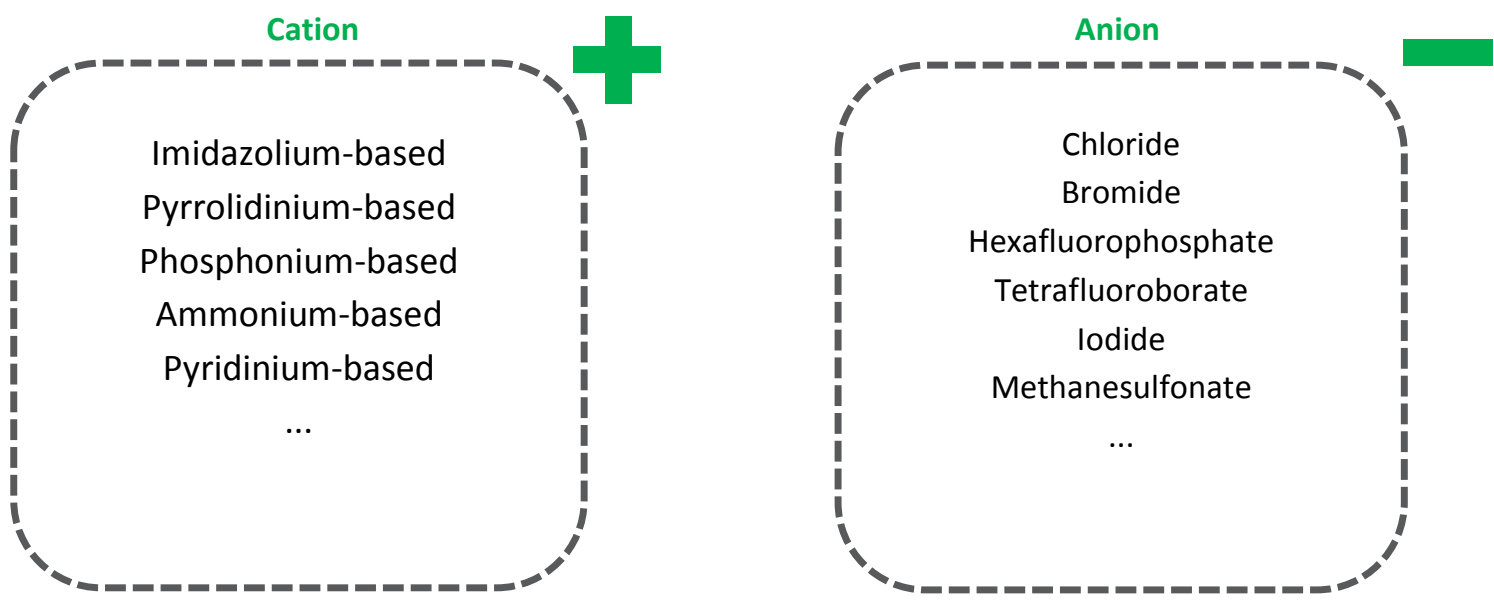

The focus on imidazolium based ILs is due to historical and practical reasons: in the early 80s, Wilkes started his pioneering work on imidazolium-based ILs. He was attracted initially by the electrochemical properties and the wide liquid range of 1-ethyl-3-methylimidazolim chloride. ${ }^{3}$ Soon after, his initial interest extended to investigating other compounds within the same family of 1,3-dialkylimidazolium-based ILs. Together with the discovery of other versatile properties, ${ }^{4-6}$ this was one of the triggers of a worldwide interest in imidazolium-based ILs in the early-mid 90s, making them the forefront family of ILs.

As stated above, physicochemical properties of ILS are tunable. Among the most interesting properties are a melting point lower than traditional salts, a wide liquid operating range, excellent thermal stability, negligible vapour pressure, high viscosity and high ionic conductivity. Thanks to these unique physicochemical properties, ILs are "multipurpose" compounds $^{7}$ and have attracted considerable interest in several branches of chemistry and chemical engineering, for instance ILs are attractive green solvents in synthetic and catalytic processes. $^{8-10}$

Less than a decade ago, Reichert et al. stated that although there is an increasing number of articles on ILs and their potential uses, their use as crystallisation solvents is poorly treated in the 
literature. ${ }^{11}$ The authors followed by explaining that commonly crystallisation is obtained by slow or fast evaporation of the solvent, and as ILs have negligible vapour pressure, chemists are cautious to investigate ILs as crystallisation solvents. ILs have a wide liquid range and are thermostable. ${ }^{9}$ Hence, ILs can be implemented as crystallisation solvents using thermal shifts and solvothermal techniques. Alternatively, other techniques such as slow diffusion, use of co-solvent and electro-crystallisation have been proposed by Reichert et al. ${ }^{11}$ Hence, ILs have found application as crystallisation media and were also proven effective in improving the crystallisation and crystal quality of macromolecules. ${ }^{12-19}$

In a special issue in CrystEngComm, where recent studies on the use of ILs in crystal engineering are exemplified, Muldon et al. nicely caught the essence of the current spirit in IL research stating: "Importantly, ionic liquids are not just replacing organic solvents, but they often give results that are very different from conventional pathways and therefore have the potential for exciting new discoveries. Despite their increasing use in inorganic and materials chemistry, there is still the need for fundamental investigations; for example, into the nature of the interactions between the ionic liquid ions and the solutes." 20

Although the attention towards practical applications of imidazolium-based ILs is still increasing, much fundamental work remains to be done on understanding their phase diagram. Our initial aim was to investigate the use of ILs as crystallisation solvents using high-pressure techniques. However we soon realised that phase diagrams of imidazolium-based ILs are either largely unexplored or poorly understood and characterised. Unveiling the solid-state behaviour of ILs was a necessary step before exploring their application as crystallisation solvents. Liquid at room temperature, ILs need external factors to crystallize such as temperature, pressure, the addition of solvents and sometimes impurity. This part of the thesis describes the use of highpressure and low-temperature techniques for studying the solid-state behaviour of imidazoliumbased ILs.

\subsection{References}

(1) Marsh, K. N.; Boxall, J. A.; Lichtenthaler, R. Room Temperature Ionic Liquids and Their Mixtures - a Review. Fluid Phase Equilib. 2004, 219, 93-98.

(2) Wilkes, J. S. A Short History of Ionic Liquids - from Molten Salts to Neoteric Solvents. Green Chem. 2002, 4, 73-80.

(3) Wilkes, J. S.; Levisky, J. A.; Wilson, R. A.; Hussey, C. L. Dialkylimidazolium Chloraluminate Melts: A New Class of Room-Temperature Ionic Liquids for Electrochemistry, Spectroscopy and Synthesis. Inorg. Chem. 1982, 21, 1263-1264.

(4) Wilkes, J. S.; Zaworotko, M. J. Air and Water Stable 1-Ethyl-3-Methylimidazolium Based Ionic Liquids. J. Chem. Soc. Chem. Commun. 1992, 965-967. 
(5) Chauvin, Y.; Mussmann, L.; Olivier, H. A Novel Class of Versatile Solvents for Two-Phase Catalysis: Hydrogenation, Isomerization, and Hydroformylation of Alkenes Catalyzed by Rhodium Complexes in Liquid 1,3-Dialkylimidazoliurn Salts. Angew. Chemie Int. Ed. English 1995, 34, 2698-2700.

(6) Dupont, J. On the Solid, Liquid and Solution Structural Organization of Imidazolium Ionic Liquids. J. Braz. Chem. Soc. 2004, 15, 341-350.

(7) Kokorin, A. Ionic Liquids : Applications and Perspectives; Kokorin, A., Ed.; InTech: Rijeka, Croatia, 2011.

(8) Earle, M. J.; Seddon, K. R. Ionic Liquids. Green Solvents for the Future. Pure Appl. Chem. 2000, 72 (7), 1391-1398.

(9) Wasserscheid, P.; Welton, T. Ionic Liquids in Synthesis, 2nd ed.; Wasserscheid, P., Welton, T., Eds.; John Wiley \& Sons: Weinheim, 2002.

(10) Pârvulescu, V. I.; Hardacre, C. Catalysis in Ionic Liquids. Chem. Rev. 2007, 107 (6), 26152665.

(11) Reichert, W. M.; Holbrey, J. D.; Vigour, K. B.; Morgan, T. D.; Broker, G. a.; Rogers, R. D. Approaches to Crystallization from Ionic Liquids: Complex Solvents-Complex Results, Or, a Strategy for Controlled Formation of New Supramolecular Architectures? Chem. Commun. (Camb). 2006, No. 46, 4767-4779.

(12) Judge, R. A.; Takahashi, S.; Longenecker, K. L.; Fry, E. H.; Abad-Zapatero, C.; Chiu, M. L. The Effect of Ionic Liquids on Protein Crystallization and X-Ray Diffraction Resolution. Cryst. Growth Des. 2009, 9 (8), 3463-3469.

(13) An, J.-H.; Kim, J.-M.; Chang, S.-M.; Kim, W.-S. Application of Ionic Liquid to Polymorphic Design of Pharmaceutical Ingredients. Cryst. Growth Des. 2010, 10 (7), 3044-3050.

(14) Hekmat, D.; Hebel, D.; Joswig, S.; Schmidt, M.; Weuster-Botz, D. Advanced Protein Crystallization Using Water-Soluble Ionic Liquids as Crystallization Additives. Biotechnol. Lett. 2007, 29 (11), 1703-1711.

(15) Pusey, M. L.; Paley, M. S.; Turner, M. B.; Rogers, R. D. Protein Crystallization Using Room Temperature Ionic Liquids. Cryst. Growth Des. 2007, 7 (4), 787-793.

(16) Hekmat, D.; Hebel, D.; Weuster-Botz, D. Crystalline Proteins as an Alternative to Standard Formulations. Chem. Eng. Technol. 2008, 31 (6), 911-916.

(17) Li, X.; Xu, X.; Dan, Y.; Feng, J.; Ge, L.; Zhang, M. The Crystallization of Lysozyme in the System of Ionic Liquid [BMIm][BF4]-Water. Cryst. Res. Technol. 2008, 43 (10), 1062-1068.

(18) Smith, K. B.; Bridson, R. H.; Leeke, G. A. Solubilities of Pharmaceutical Compounds in Ionic Liquids. J. Chem. Eng. Data 2011, 56, 2039-2043.

(19) An, J.-H.; Kim, W.-S. Antisolvent Crystallization Using Ionic Liquids As Solvent and Antisolvent for Polymorphic Design of Active Pharmaceutical Ingredient. Cryst. Growth Des. 2013, 13, 31-39.

(20) Muldoon, M. J.; Nockemann, P.; Lagunas, M. C. Crystal Engineering with Ionic Liquids. CrystEngComm 2012, 14 (15), 4873. 


\section{Chapter 7. Pinning down the solid-state polymorphism of [bmim][PF $]$}

\subsection{Synopsis}

The solid-state polymorphism of the ionic liquid 1-butyl-3-methylimidazolium hexafluorophosphate, $[\mathrm{bmim}]\left[\mathrm{PF}_{6}\right]$, has been investigated via low-temperature and high-pressure crystallisation experiments. The samples have been characterised by single-crystal X-ray diffraction, optical microscopy and Raman spectroscopy. The solid-state phase behaviour of the compound is confirmed and clarified with respect to previous phase diagrams. The structures of the previously reported $\gamma$-form, which essentially exhibits a G'T cation conformation, as well as those of the elusive $\beta$ - and $\alpha$-forms, are reported. Crystals of the $\beta$-phase are twinned and the structure is heavily disordered; the cation conformation in this form is predominantly TT, though significant contributions from other less frequently encountered conformers are also observed at low temperature and high pressure. The cation conformation in the $\alpha$-form is GT; the presence of the G'T conformer at $193 \mathrm{~K}$ in this phase can be eliminated on cooling to $100 \mathrm{~K}$. Whilst X-ray structural data are overall in good agreement with previous interpretations based on Raman and NMR studies, they also reveal a more subtle interplay of intermolecular interactions, which give rise to a wider range of conformers than previously considered.

\subsection{Introduction}

The liquid structure of ILs has been the subject of numerous theoretical and experimental investigations, the latter focusing on X-ray and neutron scattering experiments. ${ }^{1-3}$ The X-ray crystal structures of the most common ILs have also been reported in the literature: the Cambridge Structural Database ${ }^{4}$ (the CSD, V. 5.33 including updates to May 2012 was searched for structure with 3-D coordinates, an $R$-factor below $10 \%$ and no errors) contains 212 structures of 1methylimidazolium-based salts, of which 90 have a confirmed melting point below $373 \mathrm{~K}$, which is the commonly accepted maximum melting temperature for defining an IL. Crystal polymorphism in 1,3-dialkylimidazolium-based ILs has been reported on the basis of spectroscopic, diffraction and calorimetric data. To the best of our knowledge, the first example also confirmed by singlecrystal X-ray diffraction was that of $[\mathrm{bmim}] \mathrm{Cl} .{ }^{5}$

1-Butyl-3-methylimidazolium hexafluorophosphate, [bmim][ $\left.\mathrm{PF}_{6}\right]$, (Figure 7.1), is one of the most studied ionic liquids. A wide range of simulations have been undertaken on this compound and show significant probability of finding anions around the $\mathrm{C} 2$ hydrogen position as well as above and below the plane of the imidazolium ring. ${ }^{6,7}$ As found with other ionic liquids, such as the analogous chloride based systems, ${ }^{8,9}$ steric hindrance from the long alkyl chain, in this case butyl, has an effect on the structure with the probability of anion interaction with the other ring 
hydrogens being dominated by the one closest to the methyl group. The solid-state behaviour of $[\mathrm{bmim}]\left[\mathrm{PF}_{6}\right]$ at ambient-pressure conditions has been extensively studied by a variety of techniques, including Raman and NMR spectroscopy, calorimetry, single-crystal X-ray diffraction, as well as wide-angle $\mathrm{X}$-ray scattering. $[\mathrm{bmim}]\left[\mathrm{PF}_{6}\right]$ has also been the subject of high-pressure Raman and calorimetry experiments. Despite the evidence for crystal polymorphism, only one crystal structure has been reported in the literature to date. ${ }^{10,11} \mathrm{~A}$ brief summary of the findings to date is given in Table 7.1, emphasising the conditions under which different phases have been obtained and using the scheme introduced by Endo et al. ${ }^{12}$ as reference to harmonising the polymorph nomenclature.
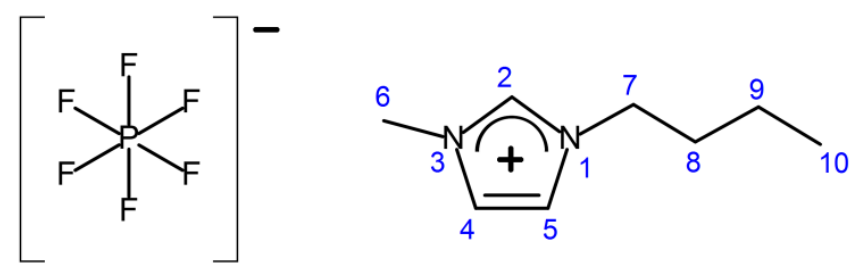

Figure 7.1. Chemical diagram of $[b \mathrm{mim}]\left[\mathrm{PF}_{6}\right]$ with atom numbering scheme

Endo et al..$^{12,13}$ characterised three crystalline forms, $\alpha, \beta$ and $\gamma$, by calorimetry, Raman and NMR spectroscopy, pointing out that the endothermic transition to the $\gamma$-phase is difficult to detect by DSC measurements. The authors assigned the cation structure in the three phases by comparing experimental Raman spectra with calculated ones obtained by full geometry optimisation, using DFT methods, of the three most stable rotational isomers of the $[\mathrm{bmim}]^{+}$cation, GT, TT and G'T (see Results and discussion section for details). The isomers were identified on the basis of the conformations found in experimental crystal structures and in gas-phase quantum chemical calculations. ${ }^{14}$ According to their Raman spectra, Endo et al. assigned the conformers of the $\alpha-, \beta$ and $\gamma$-phases as GT, TT and G'T, respectively.

The most recent and comprehensive high-pressure investigation into the polymorphism of [bmim] $\left[\mathrm{PF}_{6}\right]$ was carried out by means of Raman spectroscopy up to $c a .1$ GPa by Russina et al. ${ }^{15}$ Raman work was also carried out by Yoshimura et al. ${ }^{16}$ and by Su et al. up to $2 \mathrm{GPa} ;{ }^{17} \mathrm{Su} e t$ al. have also carried out differential thermal analysis experiments up to 1 GPa. ${ }^{18}$ Russina et al. identified two high-pressure phases by Raman spectroscopy and constructed an elegant phase diagram; at ambient temperature, they determined a crystallisation pressure of $c a .0 .048$ GPa for the phase corresponding to the $\beta$-polymorph of Endo et al., in excellent agreement with a value of ca. $0.04 \mathrm{GPa}$ reported by acoustic measurements. ${ }^{19} \mathrm{~A}$ second high-pressure phase, which the authors obtained by ambient-temperature crystallisation above ca. $0.44 \mathrm{GPa}$, was assigned the GT conformer and identified as the $\alpha$-phase of Endo et al. The preference of this conformer at high 
pressure was confirmed in a very recent molecular dynamics simulation study, albeit in the liquid state. ${ }^{20}$

Table 7.1. Summary of crystallisation conditions and observed polymorphs of [bmim][PF6] reported in the literature.

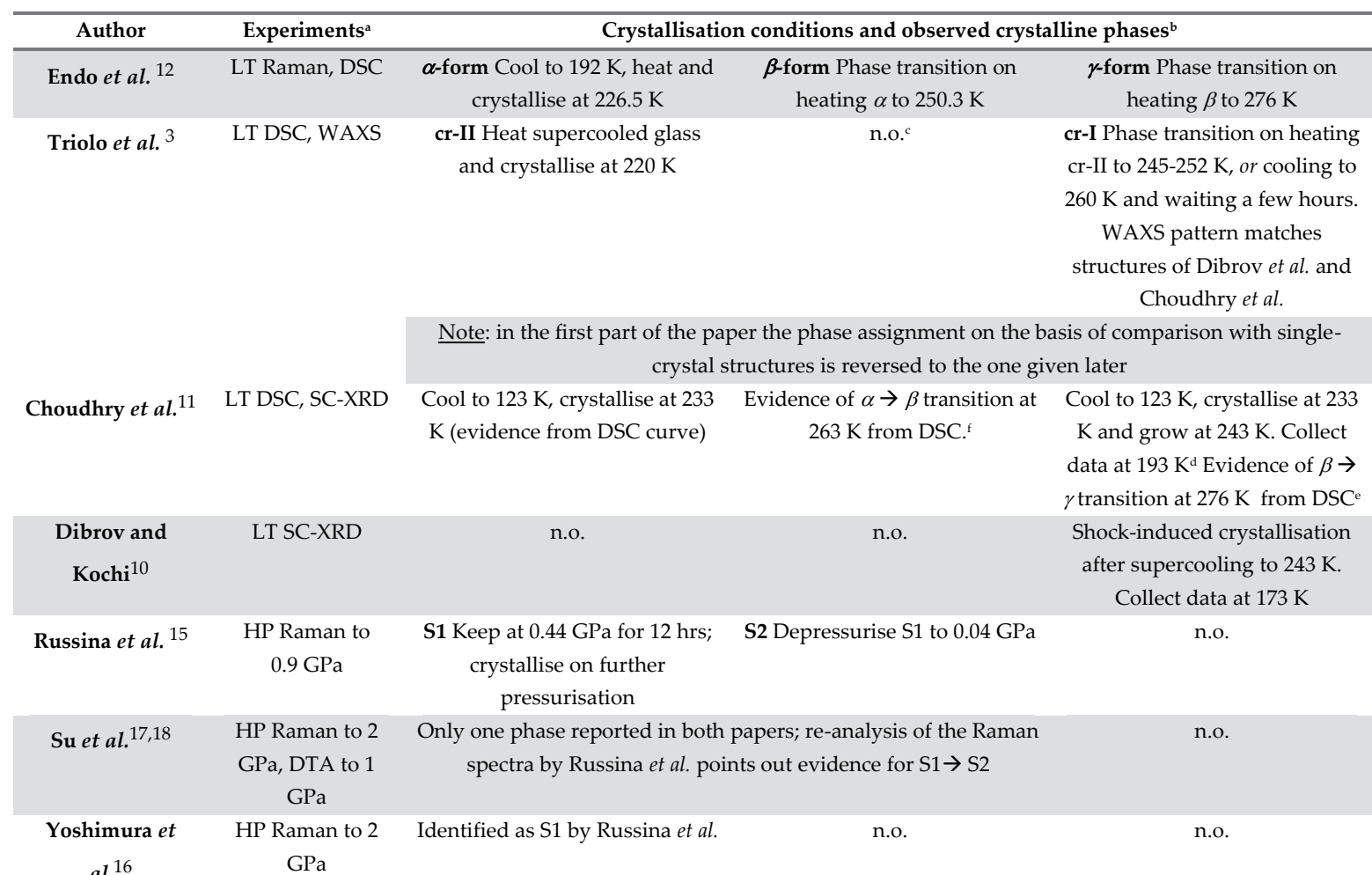

\footnotetext{
This work $\quad$ HP Raman and $\quad \alpha$-form Flash-cool below glass $\quad \beta$-form Phase transition on $\quad \gamma$-form Phase transition on XRD to $0.8 \mathrm{GPa}$; transition, heat and crystallise heating $\alpha$ to $248 \mathrm{~K}$. Crystallise heating $\alpha$ or $\beta$ between 248 and LT Raman and $\quad$ at $228 \mathrm{~K}$. Compression of $\beta$ - at high pressure below $0.4 \mathrm{GPa} \quad 258 \mathrm{~K}$; isothermal SC-XRD; optical form above $0.4 \mathrm{GPa} \quad$ crystallisation of the liquid at microscopy $\quad 277 \mathrm{~K}$. Crystallise from an aq. mix at $0.8 \mathrm{GPa}$

${ }^{a} \mathrm{P} L T=$ low temperature; HP = high pressure; DSC = differential scanning calorimetry; WAXS = wide-angle X-ray scattering; SC-XRD: singlecrystal X-ray diffraction; DTA $=$ differential thermal analysis; $b$ Bold-face phases identified in original publications; ${ }^{c}$ n.o $=$ not observed/reported; ${ }^{d}$ current interpretation of DSC signal.
}

Intrigued by the observations that polymorphism of $[\mathrm{bmim}]\left[\mathrm{PF}_{6}\right]$ was reported at both low-temperature and high-pressure conditions yet only one crystal structure is available in the CSD, our approach has been to explore how the high-pressure and low-temperature crystallisation of $[\mathrm{bmim}]\left[\mathrm{PF}_{6}\right]$ might lead to the formation of new polymorphs and to elucidate the crystal structures of these polymorphs by single-crystal X-ray diffraction, complementing the results with Raman spectroscopy. 


\subsection{Experimental}

\subsubsection{Material}

The title compound was acquired from our collaborators Dr. Norman and Prof. Hardacre (School of Chemistry and Chemical Engineering, The QUILL Centre, Queen's University Belfast, Belfast BT9 5AG, UK) who prepared it as follows: Bromobutane (1.1 eq.) was added to a stirred solution of methyl imidazole (1 eq.) in acetonitrile. The resulting mixture was stirred at $343 \mathrm{~K}$ overnight. After cooling, the solvent was concentrated and the crude product was recrystallised from ethyl acetate to yield a pale yellow solid. The bromide salt was then redissolved in acetonitrile and potassium hexfluorophosphate (1.1 eq.) was added and the suspension was stirred vigorously overnight. The mixture was then filtered and the solvent concentrated in vacuo. The resulting oil was then redissolved in DCM and refiltered to remove the $\mathrm{KBr}$ byproduct. Finally, the DCM solution was flushed through a pad of silica/alumina and charcoal and concentrated in vacuo to yield a colourless liquid. The ${ }^{1} \mathrm{H},{ }^{31} \mathrm{P}$ and ${ }^{13} \mathrm{C}-\mathrm{NMR}$ were consistent with previous reports. The ionic liquid was dried for $4 \mathrm{~h}$ at $333 \mathrm{~K}$ under high vacuum before use.

\subsubsection{High-pressure crystallisation: $\alpha$ - and $\beta$-phases}

A square-shaped beryllium-free diamond-anvil cell (DAC) of the Ahsbahs type ${ }^{21}\left(45^{\circ}\right.$ halfcell opening angle) was used for the high-pressure experiments; the cell was equipped with 600 $\mu \mathrm{m}$ culet diamonds of low fluorescence grade and Inconel gaskets with a starting diameter hole of $300 \mu \mathrm{m}$. The pressure inside the sample chamber was measured according to the ruby fluorescence method $^{22}$ using an in-house built kit that has a precision of $0.05 \mathrm{GPa}$. Crystallisation was induced by increasing the pressure progressively to $c a .0 .7 \mathrm{GPa}$. A single crystal was obtained by slow melting of the resulting polycrystalline powder when releasing the pressure to ca. $0.09 \mathrm{GPa}$ (isothermal crystal growth) and subsequently increasing the pressure until the crystal was as big as the gasket hole (Figure 7.2). The final pressure inside the sample chamber was $0.35 \mathrm{GPa}$. A second single crystal was obtained by cooling the loaded DAC at $0.4 \mathrm{GPa}$ for $12 \mathrm{~h}$ at $277 \mathrm{~K}$ and subsequently warming it to room temperature and adjusting the pressure for optimal crystal growth. The final crystal at $c a .0 .07 \mathrm{GPa}$ was in equilibrium with the liquid and X-ray diffraction data were collected at phase boundary conditions (Figure 7.2). Both single crystals were attributed to the $\beta$-form. Despite the larger standard uncertainty, our value for the crystallisation pressure is in good agreement with that of $0.048(25)$ GPa by Russina et al. ${ }^{15}$ On compressing the single crystals to pressures above ca. $0.4 \mathrm{GPa}$, a phase transition accompanied by the disintegration into polycrystalline material was observed. Analysis of this phase at 0.8 GPa by Raman spectroscopy revealed the presence of the $\alpha$-phase. 


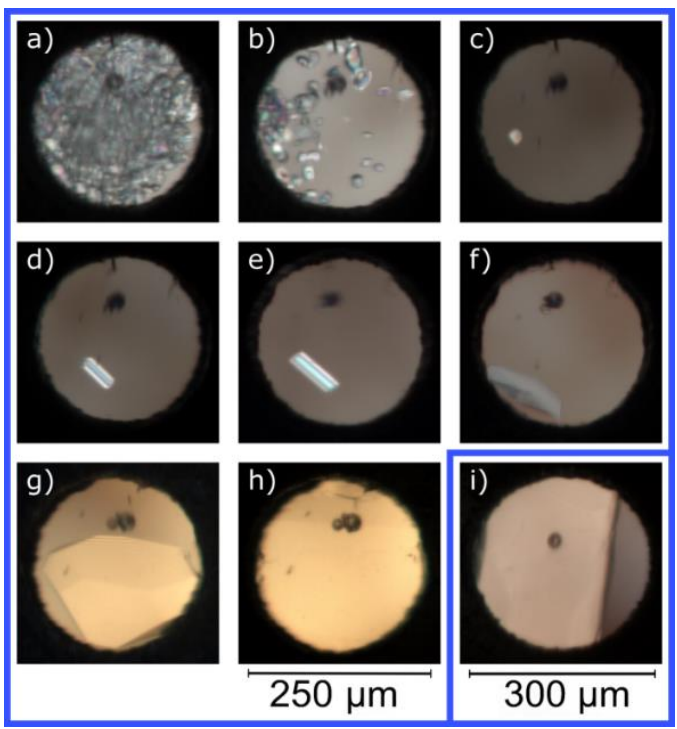

Figure 7.2. Stages of isothermal in situ crystal growth of the $\beta$-phase of [bmim][PF $]$ in the DAC at $293 \mathrm{~K}$. (a) and (b): melting at ca. $0.09 \mathrm{GPa}$; (c)-(g): slow growth on increasing pressure; (h): final crystal at $0.35 \mathrm{GPa}$ (crystal 1); (i): final crystal at $0.07 \mathrm{GPa}$ (crystal 2) in equilibrium with the liquid phase.

\subsubsection{Crystallisation in capillaries: $\alpha$ - and $\beta$-phases}

Initial crystallisation experiments were performed by loading the sample in $0.3 \mathrm{~mm}$ glass capillaries. The capillaries were sealed to keep the sample dry. The sample was rapidly flash cooled by immersing the capillary in liquid nitrogen to induce the crystallisation of a glass. On warming, a liquid-mediated transition to needle-like crystals was observed; on further warming these needles transformed to plate-like crystals. A single crystal was then grown by temperature cycling; the capillary was subsequently transferred to a Bruker Apex diffractometer equipped with a lowtemperature device set to a temperature of $193 \mathrm{~K}$, which was chosen on the basis of the data collection temperature reported for the known crystal form. Warming similarly grown crystals to room temperature did not result in the formation of another phase. Since the sample was cooled by contact with a cotton bud soaked in liquid nitrogen, heating and cooling rates could not be monitored but were generally fast.

\subsubsection{Crystallisation using a Linkam stage: $\alpha$ - $\beta$ - and $\gamma$-phases}

To improve the temperature control, we switched to a Linkam THMS600 heating and freezing stage, which enables temperature control within a precision of $0.1 \mathrm{~K}$ while still being able to view the sample through a quartz glass window (Figure 7.3). Prior to sample cooling, the stage was heated to $373 \mathrm{~K}$ to remove any moisture trapped inside the sample chamber. Phase assignment was performed by a combination of single-crystal X-ray diffraction and Raman spectroscopy. Over 50 crystallisation trials were performed. Depending on the starting crystalline phase, several transition paths are possible: herein, we detail five crystallisation protocols. (1) The $\alpha$-form was reproducibly obtained by cooling the sample below its glass transition temperature and then 
heating it up to a temperature in the range of $228-248 \mathrm{~K}$, irrespective of the heating rate. Once formed, fast heating rates suppressed any further transition. (2) Starting from the $\alpha$-phase and using a slow heating rate $\left(0.5 \mathrm{~K} \mathrm{~min}^{-1}\right)$ a transition to the $\beta$-phase occurred at $c a .248 \mathrm{~K}$, in good agreement with the value of $250.3 \mathrm{~K}$ reported by Endo et al. ${ }^{12}$ (3) Starting from a fully crystalline sample of the $\alpha$-phase (i.e. in the absence of any liquid), following an isothermal run at $258 \mathrm{~K}$ for 10-20 min, a transition to the $\gamma$-phase was sometimes observed. (4) The $\gamma$-phase was, at times, also obtained directly from the $\beta$-form using a similar isothermal run. (5) The $\gamma$-phase, which has the lowest crystal growth rate, was reproducibly obtained by leaving the sample at $277 \mathrm{~K}$ for a few days. As reported by Endo et al.,13 the activation energy of the $\beta \rightarrow \gamma$ phase change is considerable; hence, a long isothermal phase may be a key factor for either direct nucleation of the $\gamma$-phase from the liquid state or for the $\beta \rightarrow \gamma$ transition. Considerations on the reproducibility of the crystallisation protocols are further explored in the Results and discussion section.

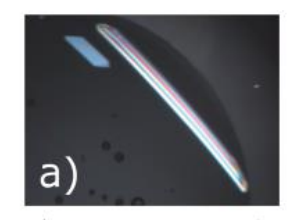

$0.8 \mathrm{~mm}$

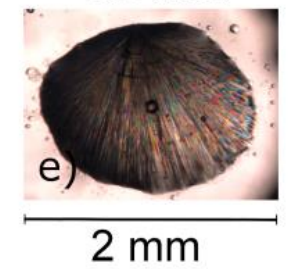

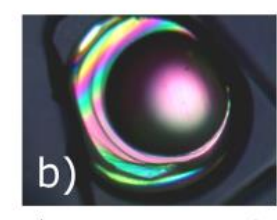

$0.8 \mathrm{~mm}$

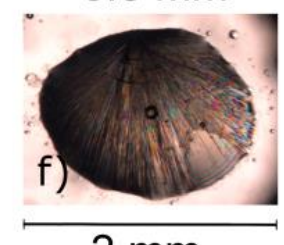

$2 \mathrm{~mm}$
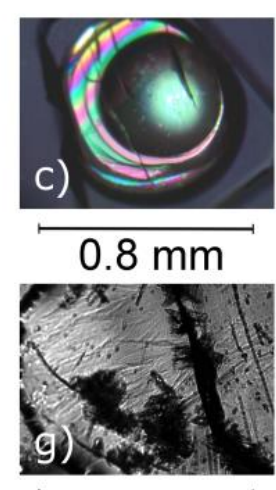

$0.8 \mathrm{~mm}$

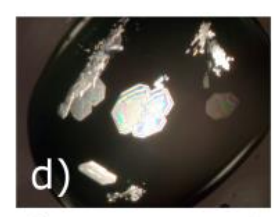

$0.8 \mathrm{~mm}$

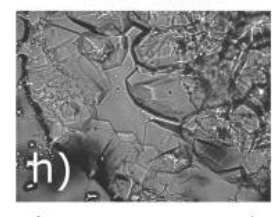

$0.8 \mathrm{~mm}$

Figure 7.3. Optical images of crystals of [bmim][PF$]$ grown on a Linkam stage. (a) Single crystals of the $\alpha$ -

phase growing from the liquid at $262 \mathrm{~K}$; (b) single crystal of the $\alpha$-phase at $260 \mathrm{~K}$ grown on a glass shard (coexistence of liquid and solid), and (c) crystal after cooling to $203 \mathrm{~K}$; (d) $\beta$-phase melting at $279 \mathrm{~K}$; (e) initial and (f) final stages of the $\alpha \rightarrow \beta$ phase transition at $248 \mathrm{~K} ;(\mathrm{g})$ nucleation of the $\gamma$-phase from the $\beta$-phase at 258 $\mathrm{K}$ and (h) $\gamma$-phase coexisting with the melt at $282 \mathrm{~K}$.

\subsubsection{Single-crystal X-ray diffraction}

Data integration and global-cell refinement were performed using the program SAINT.23 High-pressure data processing was performed according to the procedure by Dawson et al. ${ }^{24}$ Unless otherwise specified the program $S A D A B S^{25}$ was used to correct for absorption and systematic errors. For the high-pressure data, the program $S H A D E^{26}$ was additionally used. All structures were solved with direct methods using SHELXS. ${ }^{27}$ All non-H atoms were refined with anisotropic displacement parameters. All $\mathrm{H}$-atoms were placed geometrically and allowed to ride on the parent atoms. $\mathrm{U}_{\text {iso }}(\mathrm{H})$ values were assigned in the range 1.2-1.5 times $\mathrm{U}_{\text {eq }}$ of the parent atom. Full-matrix least-squares structure refinement against $\mathrm{F}^{2}$ was performed using SHELXL5 through the SHELXLE GUI. ${ }^{28}$ Crystallographic data are reported in Table 7.2. 


\subsubsection{1. $\alpha$-Phase}

Sample transfer to the diffractometer proved to be particularly difficult for this phase as any mechanical interference with the crystal at high enough temperatures where the crystal could be manipulated resulted in a phase transition. A suitable crystal for diffraction was hence grown on a secondary glass shard support placed on the Linkam stage and the shard was subsequently mounted on a Bruker SMART 6000 Apex II CCD diffractometer (Figure 7.3). Diffraction data were collected using $\mathrm{Cu} \mathrm{K} \alpha$ radiation of $\lambda=1.54178 \AA$ from a rotating anode at 100(2) and 193(2) K. The structure at $100 \mathrm{~K}$ was found to be ordered. At $193 \mathrm{~K}$ disorder could be modelled for the $\left[\mathrm{PF}_{6}\right]^{-}$ anion [0.44(2): 0.56(2)] and for the atoms C8 and C9 of the butyl side chain [0.752(13):0.248(13)] using two-site split models. To ensure reasonable anion geometry, restraints on bond distances and angles as well as on anisotropic displacement parameters were used. Distance restraints were also used for the disordered atoms in the cation.

\subsubsection{2. $\beta$-Phase}

Diffraction data were collected using a Bruker AXS SMART Apex II CCD diffractometer equipped with Mo K $\alpha$ sealed-tube radiation of $\lambda=0.71073 \AA$ for an in situ grown crystal contained in a capillary at 193(2) K. A similar diffractometer equipped with a Ag microsource (Incoatec) of $\lambda=0.56085 \AA$ a was used for the 293(2) K high-pressure DAC experiment. Both low-temperature and high-pressure structures were found to be pseudomerohedral triclinic twins, with unit-cell constants emulating a monoclinic $C$ metric cell and a $\left[\begin{array}{lll}-1 & 1 & 0\end{array}\right]$ twin direction; the twin law is

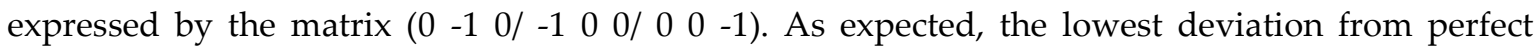
monoclinic C symmetry was observed for the high-pressure structure collected at phase-boundary conditions and this is also the structure exhibiting the highest degree of pseudosymmetry and perfect domain overlap. Further compression of the single crystal to ca. $0.35 \mathrm{GPa}$ resulted in a unit cell with a higher degree of deviation from a perfect monoclinic $C$ cell [triclinic unit-cell parameters: $a=9.4360(15), b=9.4899(8), c=14.4658(8) \AA, \alpha=98.944(5), \beta=98.754(9), \gamma=$ $\left.100.586(9)^{\circ}\right]$. Two orientation matrices were used to integrate the low-temperature data, for which the two twin components did not perfectly overlap; the program TWINABS ${ }^{29}$ was used to correct for absorption and systematic errors and a HKLF5 reflection file was used for the refinement of the low-temperature structure. The twin scale factors were $0.445(5)$ and $0.509(18)$ for the lowtemperature and high-pressure crystals, respectively. The octahedral geometry of the F-atoms was enforced through the use of restraints whilst opposite F-atoms were constrained to have the same anisotropic displacement parameters. For the high-pressure structure, disordered C-atoms were refined with isotropic displacement parameters. Both $\left[\mathrm{PF}_{6}\right]^{-}$anions were found to be disordered. A two-component disorder model was refined for each of these groups with occupancy factors refining to approximately 50:50 values at both low temperature and high pressure (occupancies 
were fixed to these values for the high-pressure refinement). Disorder was additionally found in the butyl side chain of the cations: at low temperature disorder was clearly identifiable for carbon $\mathrm{C} 10$ in one of two symmetry-independent molecules [0.68(4) : 0.32(4)]. At high pressure terminal methyl carbons were disordered in both molecules [site occupancies were fixed to $0.7: 0.3$ after initial refinement]. Distance, angle, as well as rigid-bond (DELU) and thermal similarity (SIMU) restraints were applied to ensure reasonable molecular geometries and ADPs and increase the data to parameter ratio, in particular for the high-pressure data. In addition, the independent molecules in the asymmetric unit were restrained to have similar geometries. The preference for using a heavily restrained anisotropic model over the use of an isotropic model with fewer parameters for crystals whose weak diffraction can be ascribed to large thermal motion has been discussed by Watkin. ${ }^{30}$

Table 7.2. Crystallographic data for the three polymorphs of [bmim][PF 6 discussed in this chapter.

\begin{tabular}{|c|c|c|c|c|c|c|}
\hline Structure & $\alpha$ & $\alpha$ & $\beta$ & $\beta$ & $\gamma$ & $\gamma$ \\
\hline$T / K$ & $100(2)$ & $193(2)$ & $193(2)$ & $293(2)$ & $180-193^{a}$ & $263(2)$ \\
\hline Reference & This work & This work & This work & This work & MAZXOB017 & This work \\
\hline Space group & $\mathrm{Pbca}$ & $\mathrm{Pbca}$ & $P-1$ & $P-1$ & $P-1$ & $P-1$ \\
\hline$a / \AA$ & $9.3855(3)$ & $9.4924(5)$ & $9.3869(8)$ & $9.5818(13)$ & $8.774(5)$ & $8.8215(8)$ \\
\hline b/Å & $9.7769(3)$ & $9.8406(5)$ & $9.5879(8)$ & $9.5826(9)$ & $8.944(9)$ & $9.0796(9)$ \\
\hline$c / \AA ̊$ & $26.7170(7)$ & $26.8817(13)$ & $14.4964(12)$ & $14.5801(10)$ & $9.032(6)$ & $9.0381(8)$ \\
\hline$\alpha /^{\circ}$ & 90 & 90 & 98.492(5) & $99.219(10)$ & $95.95(2)$ & $96.671(7)$ \\
\hline$\beta /^{\circ}$ & 90 & 90 & $98.354(6)$ & $99.252(6)$ & $114.93(1)$ & $114.768(6)$ \\
\hline$\gamma^{\circ}$ & 90 & 90 & $101.089(6)$ & $99.667(10)$ & 103.01(3) & 103.071(7) \\
\hline$V / \AA^{3}$ & $2451.58(13)$ & $2511.0(2)$ & $1245.85(18)$ & $1278.2(2)$ & $610.2(8)$ & $621.82(10)$ \\
\hline$Z^{\prime}$ & 1 & 1 & 2 & 2 & 1 & 1 \\
\hline$D_{\text {calc }} / \mathrm{g} \mathrm{cm}^{-3}$ & 1.540 & 1.503 & 1.515 & 1.477 & 1.55 & 1.518 \\
\hline $\begin{array}{c}\text { Measured/unique/ } \\
\text { observed reflections }^{\mathrm{b}}\end{array}$ & $\begin{array}{c}24529,2380 \\
2254\end{array}$ & $\begin{array}{c}28075,2427, \\
2054\end{array}$ & $\begin{array}{c}18072,3544 \\
2711\end{array}$ & $\begin{array}{c}10400,1063 \\
815\end{array}$ & 6680, 3484, n.a. & $\begin{array}{c}7824,2291, \\
1624\end{array}$ \\
\hline Parameters/restraints & $156 / 0$ & $229 / 255$ & $349 / 618$ & $341 / 809$ & $154 / 0$ & $174 / 57$ \\
\hline$R_{\text {int }}$ & 0.05 & 0.06 & 0.05 & 0.04 & n.a. & 0.02 \\
\hline$R_{1}{ }^{c}$ & 0.037 & 0.048 & 0.084 & 0.074 & 0.045 & 0.050 \\
\hline$w R_{2} \mathrm{~d}$ & 0.100 & 0.155 & 0.275 & 0.2113 & 0.128 & 0.145 \\
\hline$\Delta \rho_{\min } / \Delta \rho_{\max } / \mathrm{e}^{-} \AA^{-3}$ & $-0.31 / 0.33$ & $-0.39 / 0.27$ & $-0.41 / 0.52$ & $-0.14 / 0.18$ & $-0.29 / 0.33$ & $-0.34 / 0.42$ \\
\hline
\end{tabular}

\subsubsection{3. $\gamma$-Phase}

Diffraction data were collected on a single crystal grown on the Linkam stage and transferred to a Bruker AXS SMART Apex II CCD diffractometer with Mo K $\alpha$ sealed-tube radiation of $\lambda=0.71073 \AA$ at 263(2) K. A minor but significant disordered component of the butyl side chain, atoms C8 and C9, could be successfully refined using a split-site model [0.921(8):0.079(8)]. The residual electron density was distributed around the anion but no disorder could be modelled satisfactorily; the resulting anisotropic displacement parameters are not unusually large for a $263 \mathrm{~K}$ structure. 


\subsubsection{Raman spectroscopy}

Raman spectra were recorded with a Horiba Jobin Yvon HR800 UV Micro-Raman spectrometer equipped with an air-cooled $20 \mathrm{~mW} 488 \mathrm{~nm}$ Ar-laser. Raman spectra were collected in the $200-1200 \mathrm{~cm}^{-1}$ range with a spectral resolution of $c a .2 .2 \mathrm{~cm}^{-1}$ using a grating of 600 grooves per mm and a Peltier-cooled CCD detector (Andor, $1024 \times 256$ pixels). The spectra were calibrated with the Raman scattering frequency of Si before and after each measurement.

\subsection{Results and discussion}

\subsubsection{Conformation of the $[\mathrm{bmim}]^{+}$cation in the solid state}

In the present discussion, the rotamer denomination follows the scheme outlined by Tsuzuki et al., ${ }^{14}$ which is based on the $\mathrm{C} 2-\mathrm{N} 1-\mathrm{C} 7-\mathrm{C} 8$ torsion angle (Figure 7.1) being positive. The conformations of the $\mathrm{N} 1-\mathrm{C} 7-\mathrm{C} 8-\mathrm{C} 9$ and $\mathrm{C} 7-\mathrm{C} 8-\mathrm{C} 9-\mathrm{C} 10$ torsion angles, $\theta_{1}$ and $\theta_{2}$, respectively, are then assigned to the following angle ranges: $\mathrm{T}\left(\right.$ trans, -150 to $\left.+150^{\circ}\right), \mathrm{G}\left(\right.$ gauche,+30 to $\left.+90^{\circ}\right)$ and $\mathrm{G}^{\prime}$ (gauche', -30 to $-90^{\circ}$ ), see Figure 7.5 for details. As noted by Endo et al.,12 the three polymorphs of [bmim] $\left[\mathrm{PF}_{6}\right]$ can be identified on the basis of characteristic Raman bands: for the $\alpha$-phase a band at $338 \mathrm{~cm}^{-1}$, associated with ring wagging and chain deformation; ${ }^{31}$ for the $\beta$-phase a band at $624 \mathrm{~cm}^{-1}$, which is associated with a number of group vibrational modes; ${ }^{32}$ for the $\gamma$-phase a band at $326 \mathrm{~cm}^{-1}$, presumably also associated with ring wagging and chain deformation. Endo et al. ${ }^{12}$ note that these bands are characteristic fingerprints for the GT, TT and G'T conformers, respectively. Our experimental Raman spectra, shown in Figure 7.4, are in very good agreement with those reported at low temperature by Endo et al..$^{12}$ and at high pressure by Russina et al..$^{15}$

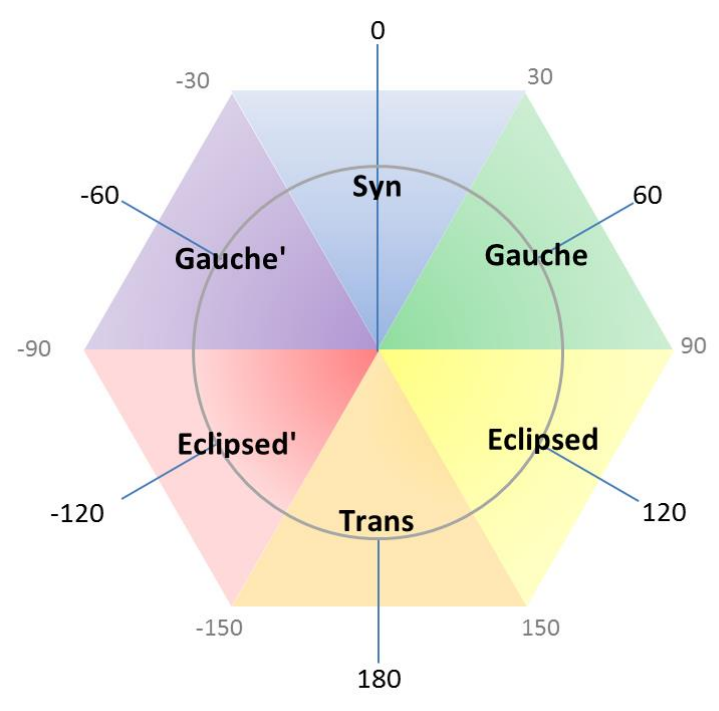

Figure 7.4. Dihedral angles for N1-C7-C8-C9 and C7-C8-C9-C10 for defining the $[\mathrm{bmim}]^{+}$cation conformation. 

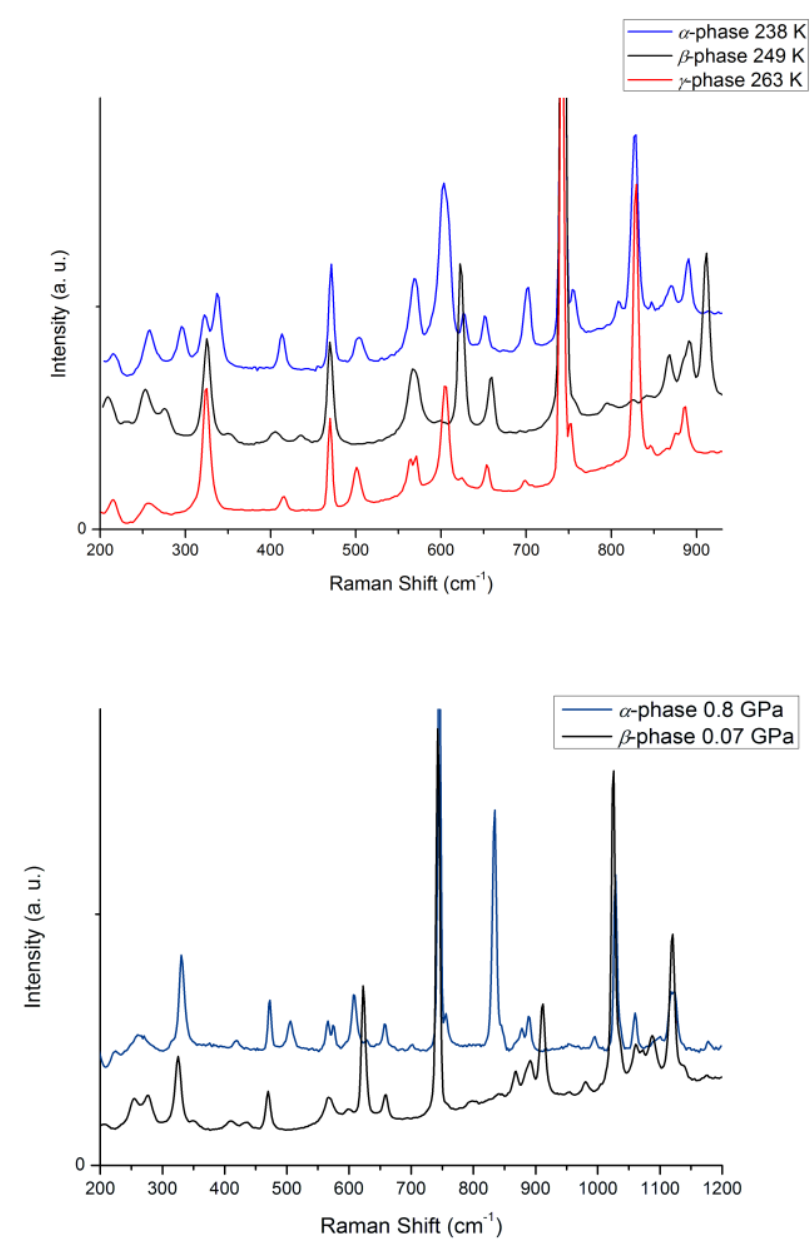

Figure 7.5. Raman spectra of [bmim][ $\left.\mathrm{PF}_{6}\right]$ polymorphs collected at different conditions of temperature (top) and pressure (bottom). Solid lines highlight the frequencies of the characteristic bands below $800 \mathrm{~cm}^{-1}$ for the $\alpha$-, $\beta$ - and $\gamma$-forms at 338,624 and $326 \mathrm{~cm}^{-1}$, respectively.

Whilst Raman spectroscopy enables a rapid and unambiguous phase assignment, singlecrystal X-ray diffraction allows the determination of a more detailed description of the cation conformation in the solid state. Gas-phase calculations on the cation, in isolation and in the presence of counterions have shown that the nine lowest-energy rotamers adopt a combination of the $T, G$ and $\mathrm{G}^{\prime}$ torsion angles, and that the energy of all rotamers are within $5 \mathrm{~kJ} \mathrm{~mol}^{-1}$ of each other, ${ }^{14}$ however, our crystallographic study points out to the existence of other conformers, which in the previous calculations correspond to saddle points on the torsional potentials, so that additional torsion angles are introduced here: $\mathrm{S}\left(\mathrm{syn},-30\right.$ to $\left.+30^{\circ}\right)$, $\mathrm{E}\left(\right.$ eclipsed, +90 to $\left.+150^{\circ}\right)$ and $\mathrm{E}^{\prime}$ (eclipsed', -90 to $-150^{\circ}$ ). The values for the torsion angles $\theta_{1}$ and $\theta_{2}$ observed from single-crystal diffraction are summarised in Table 7.3. 
Table 7.3. Torsion angles and cation conformation for the polymorphs of [bmim][PF $]$ at different experimental conditions.

\begin{tabular}{|c|c|c|c|c|c|c|}
\hline & $\alpha-100 \mathrm{~K}$ & $\alpha-193 \mathrm{~K}$ & $\beta-193 \mathrm{~K}$ & $\beta-0.07 \mathrm{GPa}$ & $\gamma-193 \mathrm{~K}^{17}$ & $\gamma-263 \mathrm{~K}$ \\
\hline $\begin{array}{c}\theta_{1}, \mathrm{~N} 1-\mathrm{C} 7-\mathrm{C} 8-\mathrm{C} 9 /^{\circ} \\
\text { (molecule 1) }\end{array}$ & $63.65(19)$ & $\begin{array}{c}63.5(5) \\
{[-54.1(14)]^{a}}\end{array}$ & $179.8(12)$ & $\begin{array}{c}168(2) \\
{[-164(3)]}\end{array}$ & $-60.80(17)$ & $\begin{array}{l}-60.3(5) \\
{[56(4)]}\end{array}$ \\
\hline $\begin{array}{c}\theta_{1}, \mathrm{~N} 11-\mathrm{C} 17-\mathrm{C} 18-\mathrm{C} 19 /^{\circ} \\
\text { molecule } 2^{b}\end{array}$ & & & $167.5(8)$ & $168(2)$ & & \\
\hline $\begin{array}{c}\theta_{2,} \mathrm{C} 7-\mathrm{C} 8-\mathrm{C} 9-\mathrm{C} 10 /{ }^{\circ} \\
\text { molecule } 1\end{array}$ & $176.76(14)$ & $\begin{array}{c}176.2(3) \\
{[-176.0(8)]}\end{array}$ & $\begin{array}{l}-64.4(19) \\
{[-104(2)]}\end{array}$ & $\begin{array}{c}166(3) \\
{[-135(3)]}\end{array}$ & $-178.05(17)$ & $\begin{array}{r}-176.4(3) \\
{[173(3)]}\end{array}$ \\
\hline $\begin{array}{c}\theta_{2}, \mathrm{C} 17-\mathrm{C} 18-\mathrm{C} 19-\mathrm{C} 20 /{ }^{\circ} \\
\text { molecule } 2\end{array}$ & & & $180.0(8)$ & $\begin{array}{c}-178(3) \\
{[-122(4)]}\end{array}$ & & \\
\hline Conformation molecule 1 & GT & $\begin{array}{c}\text { GT } \\
\left(25 \% G^{\prime} T\right)\end{array}$ & $\begin{array}{c}\mathrm{TG}^{\prime} \\
\left(30 \% T E^{\prime}\right)\end{array}$ & $\begin{array}{c}\mathrm{TT} \\
\left(30 \% T E^{\prime}\right)\end{array}$ & $\mathrm{G}^{\prime} \mathrm{T}$ & $\begin{array}{c}\mathrm{G}^{\prime} \mathrm{T} \\
(8 \% G T)\end{array}$ \\
\hline Conformation molecule 2 & & & $\mathrm{TT}$ & $\begin{array}{c}\mathrm{TT} \\
\left(30 \% T E^{\prime}\right)\end{array}$ & & \\
\hline
\end{tabular}

The conformers of the $\alpha$ - and $\gamma$-phases are confirmed to be GT and G'T, respectively. The coexistence of the GT and G'T conformers in the $\alpha$-phase, suggested by Endo et al. ${ }^{12}$ on the basis of variable-temperature Raman spectroscopy can also be rationalised: the single-crystal structure at $193 \mathrm{~K}$ indicates the presence of a minor (25\%) component attributed to the G'T conformer. On further cooling the same crystal, the disorder disappears and the observed conformation is entirely GT. This observation points towards the presence of dynamic disorder in the cation, though ideally more data points should be collected to confirm this observation. Dynamic disorder or enhanced thermal motion in the cation is, to a lesser extent, also present in the $\gamma$-form: whilst the structures reported by Dibrov and Kochi ${ }^{10}$ and Choudhury et al. ${ }^{11}$ determined below $200 \mathrm{~K}$ indicate that the cation conformer is $\mathrm{G}^{\prime} \mathrm{T}$, our $263 \mathrm{~K}$ structure additionally shows the presence of a minor GT component (8\%). Multiple conformations are also observed for the two molecules in the asymmetric unit of the $\beta$-phase at $193 \mathrm{~K} / 0.1 \mathrm{MPa}$ and at $293 \mathrm{~K} / 0.07 \mathrm{GPa}$. When considering the main disordered components only, the conformations of the [bmim $]^{+}$cation at $193 \mathrm{~K} / 0.1 \mathrm{MPa}$ and at $293 \mathrm{~K} / 0.07 \mathrm{GPa}$ are best described as $\mathrm{TG}^{\prime} / \mathrm{TT}$ and $\mathrm{TT} / \mathrm{TT}$, respectively. When the minor disordered components are also taken into account, the description becomes $\mathrm{TG}^{\prime}\left(30 \% \mathrm{TE}^{\prime}\right) / \mathrm{TT}$ at low temperature and TT $\left(30 \% \mathrm{TE}^{\prime}\right) / \mathrm{TT}\left(30 \% \mathrm{TE}^{\prime}\right)$ at high pressure. Crystal structures of ionic liquids crystallising with more than one molecule in the asymmetric unit do in fact often contain different conformers; the E and $E^{\prime}$ conformers are considerably less common compared to the $T, G$ and $G^{\prime}$ counterparts, but not unknown. A search for $[\mathrm{bmim}]^{+}$containing structures in the current version of the Cambridge Structural Database (the CSD, V. 5.33 including updates to August 2012, was searched for structure containing the $[\mathrm{bmim}]^{+}$cation with available 3-D coordinates, an $R$-factor below $10 \%$, no errors and excluding powder structures) reveals that out of a total of 96 structures (90 unique), 1 contains twelve $[\mathrm{bmim}]^{+}$cations in the asymmetric unit, another contains five, 9 contain four, 2 contain three and 19 contain two. The distribution of the observed conformers is shown in Figure 7.6. The wide range of different conformations observed in different polymorphs 
and in the same phase at different conditions of pressure and temperature is a further indication of the low barrier to rotational isomerism. In a very recent solid-state ${ }^{1} \mathrm{H}$ NMR relaxation experiment study Endo et al. ${ }^{13}$ reported that the averaged mobility of the cation follows the order $\gamma<\beta \leq \alpha$ and that the same trend is also followed by the slow segmented motion of the butyl group. These findings are in general good agreement with our observations; moreover, in most cases the motions seem to be larger than vibrational motions and are associated with conformational changes of the butyl group.

The TT conformer assignment for the $\beta$-phase by Endo et al. and Russina et al. on the basis of Raman data is essentially correct when considering the major disorder component; however, the availability of single-crystal data provides a higher level of detail. The model presented, herein, still represents an averaged model: the data do not allow a deconvolution of electron density and thermal motion and the given values should be taken as guidelines; in reality it is possible that a range of conformations between $\mathrm{TG}^{\prime}$ and $\mathrm{TE}^{\prime}$ exist in the crystal, in particular as a function of varying temperature and pressure.

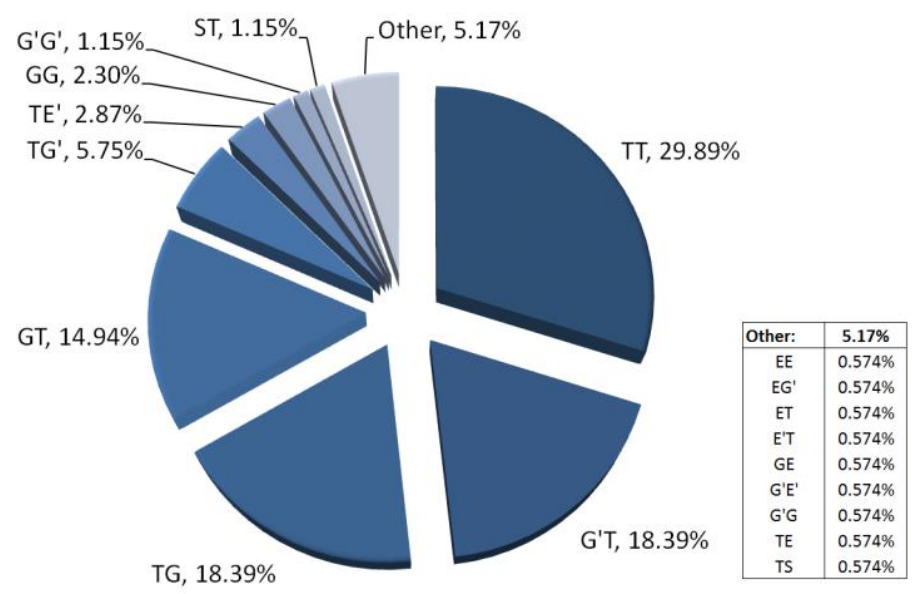

Figure 7.6. Distribution of the conformers of [bmim $]^{+}$-containing structures found in the CSD.

\subsubsection{Disorder of the $[\mathrm{PF} 6]^{-}$anion in the solid state}

Rotational disorder of the $\left[\mathrm{PF}_{6}\right]^{-}$group is often observed in the solid state, as expected for a highly symmetric molecule. At $193 \mathrm{~K}$ the only crystalline phase which does not show evidence of anion disorder is that of $\gamma$, which incidentally is also the most dense phase at this temperature. In the $\alpha$-phase the nature of the anion disorder is most likely to be dynamic, as exemplified by the ordering observed on cooling the single-crystal from 193 to $100 \mathrm{~K}$. In the $\beta$-phase at $193 \mathrm{~K}$, the two crystallographically independent anions are both extensively disordered about axial F-P-F bonds (F101-P1-F103 and F204-P11-F206 for molecules 1 and 2, respectively); at low temperature the [PF6] groups were found to be primarily disordered about an axis rotation, whilst at high pressure an additional significant tilt component was observed. A similar but less pronounced type of disorder 
is also observed in the slightly denser $\alpha$-phase at $193 \mathrm{~K}$ (about the F2-P1-F5 axis). The trends in the anion disorder in the crystalline state are in agreement with the results of the spin-lattice rotational dynamics study of Endo et al. ${ }^{13}$

\subsubsection{Crystal structures of the three polymorphs}

The structure of the $\gamma$-phase determined at $263 \mathrm{~K}$ is in very good agreement with the lowtemperature structure reported previously. The crystal packing in this form is based on two primary building blocks of centrosymmetric cation dimers. In the first, here termed "closed" dimer, the butyl groups are pointing inwards, i.e. towards the space between two imidazolium rings, and in the second, "open" dimer, they are pointing outwards (Figure 7.7). Both types of packing arrangements are found in short- and long-chain ionic liquids. ${ }^{5,33,34}$ In long alkyl structures, "closed" dimers favour hydrophobic alkyl-alkyl interactions. The $\beta$-phase also exhibits both types of packing arrangements: the "open" dimer is formed between symmetry equivalent molecules, whilst the "closed" one, a pseudo-centrosymmetric dimer, is formed between symmetryindependent molecules. In contrast to the $\gamma$ - and $\beta$-forms, the $\alpha$-phase only exhibits one type of centrosymmetric dimeric arrangement, namely the "open" dimer; in addition, a different, less compact closed dimer, with molecules related by glide symmetry, is formed. The nature of the "open" dimer also changes across the three structures: in the $\alpha$ - and $\beta$-phases the methyl groups of the imidazolium ring are aligned, whereas in the $\gamma$-form the butyl groups are. When considering the entire crystal packing, an overall change from a mixed short-alkyl/long-alkyl to a long-alkyl structure-type packing arrangement is observed when going from the $\gamma$-through the $\beta$ - to the $\alpha$ phase. The phase that crystallises at the lowest temperature from the supercooled liquid state adopts a packing arrangement that is more akin to that encountered in long-alkyl chain hexafluorophosphate ionic liquids (Figure 7.8), with layers of interacting butyl side chains separated by $c / 2$, similar to what was previously predicted for this compound..$^{34}$ This is in line with the thermodynamic data of Triolo et al., ${ }^{35}$ who determined that the supercooled liquid-fragility index of $[\mathrm{bmim}]\left[\mathrm{PF}_{6}\right]$ is similar to that of longer side chain 1-alkyl-3-methyl imidazolium-based salts. ${ }^{36}$ 


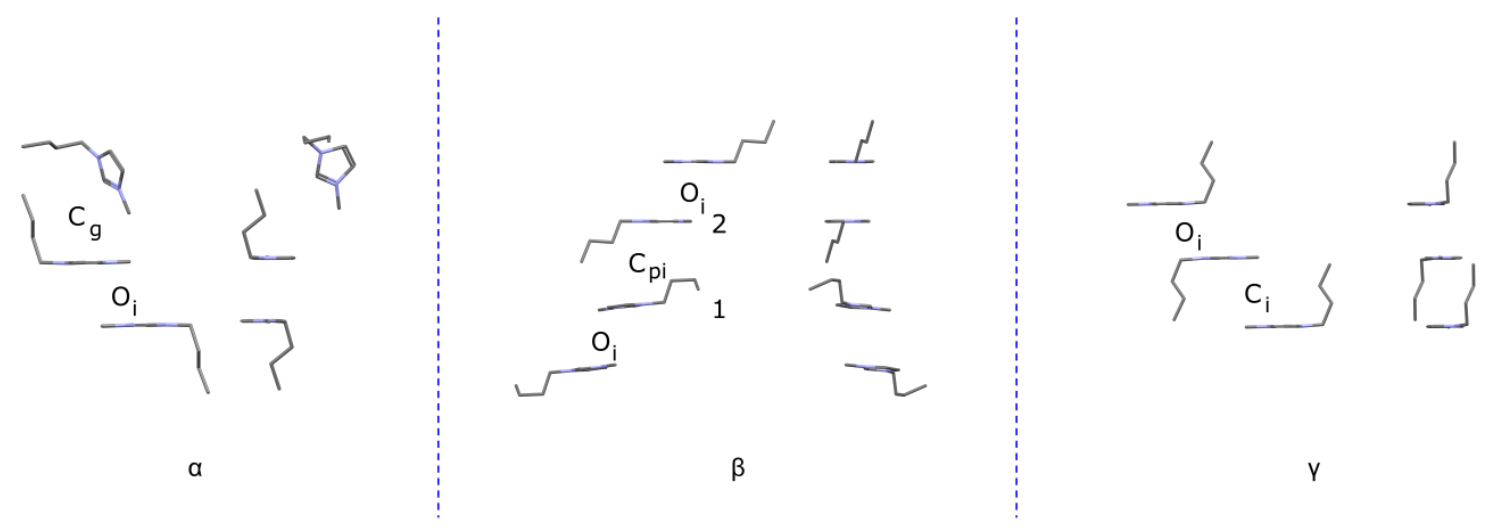

Figure 7.7. Dimeric arrangements in the three polymorphs of [bmim][PF$]$ viewed along two different stacking directions. Anions, $\mathrm{H}$-atoms and minor disorder components have been omitted for clarity. $\mathrm{O} \mathrm{i}=$ centrosymmetric "open" dimer, $\mathrm{c}_{\mathrm{i}}=$ centrosymmetric "closed" dimer, $\mathrm{c}_{\mathrm{pi}}=$ pseudo-centrosymmetric "closed" dimer, $\mathrm{cg}=$ glide "closed" dimer. Symmetry-independent molecules in the $\beta$-phase are labelled 1 and 2.
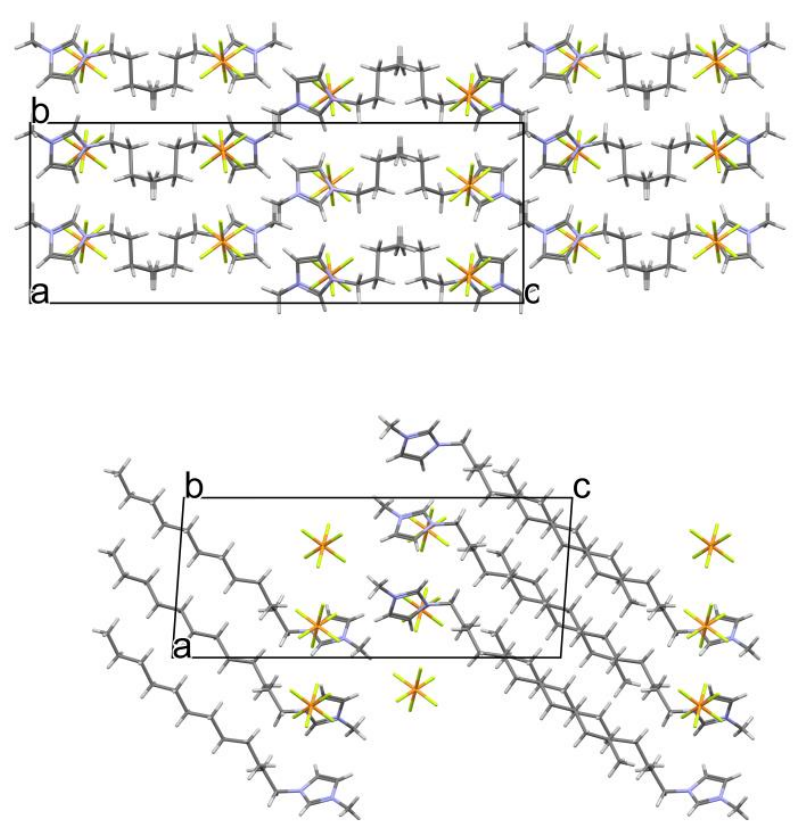

Figure 7.8. Crystal packing of [bmim][PF/ $]$. $\alpha$-form (top) and of 1-n-dodecyl-3-methylimidazolium hexafluorophosphate, ${ }^{34}$ CSD Ref. code HIWNOQ (bottom).

The geometric parameters describing the centrosymmetric dimeric arrangements are detailed in Table 7.4 (see Appendix for details). $\pi-\pi$ stacking of cation dimers is observed between dimers in the "open" arrangement, and the smallest offset to perfect stacking is found in the $\beta$ phase [2.342 $\AA$ at $193 \mathrm{~K}$ compared to 3.543 and $3.394 \AA$ in the $\alpha$ - and $\gamma$-phases, respectively, at the same temperature], although the interplanar distance is also the longest. At $193 \mathrm{~K}$, the interplanar distance for the "closed" dimer is shortest in the $\gamma$-phase [4.3688(9) $\AA$ compared to 5.452(4) $\AA$ in the $\beta$-phase]. From Table 7.4 the more pronounced pseudo-symmetry in the $\beta$-form at high pressure is 
evident: all parameters describing the "open" dimers between symmetry inequivalent molecules are more similar at high pressure than they are at low temperature.

The $\left[\mathrm{PF}_{6}\right]^{-}$anions contribute to structural stability by linking the dimeric arrangements via several F $\cdots \mathrm{H}$ contacts. In the structures of the $\gamma$ - and $\beta$-phases, the anions occupy the space between the stacked cation columns, whilst in the structure of the $\alpha$-phase the structure motifs formed by cations and anions are intertwined. Void analysis (performed with the program Mercury $^{37}$ ) reveals that whilst this space forms continuous columns along the $b$-axis in the $\gamma$-form, it gradually moves into a double-pocket arrangement and finally into segregated individual pockets that fit a single anion on going from $\gamma$ to $\beta$ and from $\beta$ to $\alpha$ (Figure 7.9).

Interestingly, at $0.07 \mathrm{GPa}$ the density of the $\beta$-phase is $c a .2 .5 \%$ less than the corresponding density of the same phase at $193 \mathrm{~K}$. Isothermal compression at $293 \mathrm{~K}$ to $c a .0 .35 \mathrm{GPa}$, leads to a density increase of $c a .3 .4 \%$ from 1.477 to $1.527 \mathrm{~g} \mathrm{~cm}^{-3}$ (full structural data not reported here). The effect of decreasing temperature on the crystals of the $\gamma$ - and $\alpha$-phases is to form denser structures. The considerable variation of density as a function of temperature is indicative of the "softness" of the intermolecular interactions that govern crystal packing.

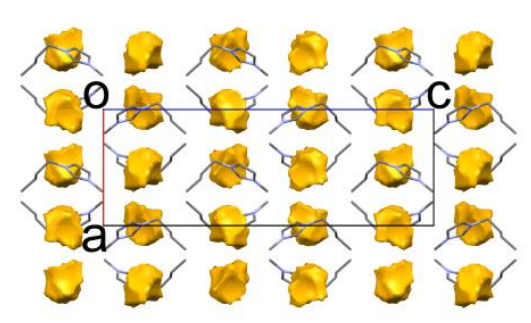

$\alpha$

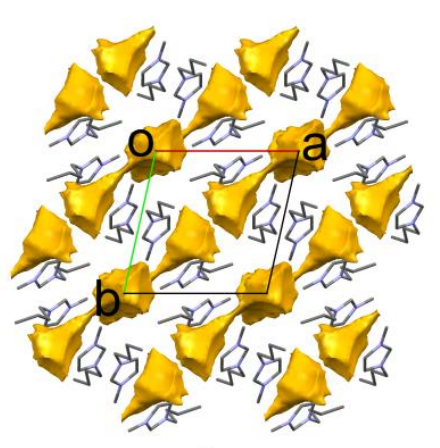

$\beta$

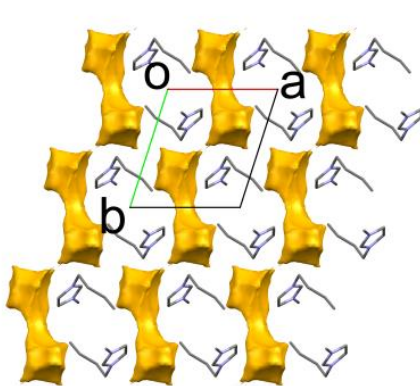

Y

Figure 7.9. Crystal packing in the three polymorphs of [bmim][PF6]. H-atoms and disordered atoms have been omitted for clarity. The space occupied by the anions is depicted by solvent accessible void surfaces generated with the program Mercury (probe radius $=1.2 \AA$, grid spacing $=0.7 \AA$ ). Solvent accessible void volume at $193 \mathrm{~K}$ expressed as \% unit cell volume: $5.1 \%$ in the $\alpha-, 5.3 \%$ in the $\beta$ - and $4.4 \%$ in the $\gamma$-polymorph, respectively.

The attractive energy between imidazolium-based cations and anions is predominantly attributable to Coulombic forces. The contact involving the acidic $\mathrm{C} 2-\mathrm{H} 2$ bond and the anion is generally accepted as a hydrogen bond, albeit weak (donor-acceptor distance higher than $3 \AA$ ) in the case of $\left[\mathrm{PF}_{6}\right]^{-} .38,39$ Tsuzuki et al., have shown by computational methods that hydrogen bonds in ionic liquids involving imidazolium ring hydrogens are not directional and are mainly governed by the distance between the ring hydrogen and the anion. In a subsequent publication, the authors have shown that a contact between a $\mathrm{Br}^{-}$anion and the $\mathrm{C} 2-\mathrm{H} 2$ group stabilises the GT $[\mathrm{bmim}]^{+}$cation conformer, whilst close contacts to $\mathrm{C} 4-\mathrm{H} 4$ and $\mathrm{C} 5-\mathrm{H} 5$ do not alter the relative 
stability of the GT, G'T and TT conformers. ${ }^{14} \mathrm{H} \cdots \mathrm{F}$ contacts less than the sum of the van der Waals radii are given in Table 7.5 (see Appendix for details). From this table it can be seen that the coordination number of the cation and anion varies across temperature, pressure and crystal phase. Within the same phase, the coordination numbers are inversely proportional to crystal density; the higher density observed at low temperature is achieved by an increase in the number of close $\mathrm{H} \cdots \mathrm{F}$ contacts, defined as contacts whose distance is smaller than the sum of the van der Waals radii. The crystal density at $193 \mathrm{~K}$ follows the order $\alpha<\beta \ll \gamma$; the same order is observed in the number of close $\mathrm{H} \cdots \mathrm{F}$ contacts, whilst the order is $\alpha \sim \gamma>\beta$ when considering both the coordination number of cations and anions. These results are in fairly good agreement with the strength of cation-anion interactions estimated from Raman spectroscopy that follows the trend $\gamma>\beta \geq \alpha .{ }^{13}$

All three crystalline phases are characterised by $\mathrm{C} \cdots \mathrm{F}$ contacts involving $\mathrm{H} 2$ to two anions on either side of the imidazolium ring. The anion is defined to lie above the ring when it is on the same side of the butyl chain and below when on the opposite side. Details of the geometric parameters for these contacts are given in the Appendix (Table 7.6). At $193 \mathrm{~K}$, short C2 $\cdots \mathrm{F}$ distances are observed for the $\alpha$-form [two contacts to disordered F-atoms at 3.062(6) and 3.064(9) $\AA$ to the anion below the ring; note that at 2.993(10) and 3.029(8) $\AA, C^{\cdots-F}$ F contacts to the anion above the ring are shorter but $\mathrm{H} \cdots \mathrm{F}$ distances are greater than the sum of the van der Waals radii]. In the $\gamma$ form the corresponding distance is slightly longer [3.192(4) $\AA$; in this form, the distance to the

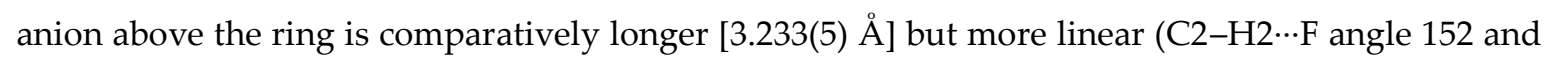
$120^{\circ}$, respectively). In the $\beta$-form a wider range of distances to anions involving $\mathrm{H} 2$ are observed because of the presence of two molecules in the asymmetric unit and extensive anion disorder, thereby making contact analysis more complex. For instance, at $193 \mathrm{~K}$ the shortest distance has a value of 2.921(16) $\AA$ and involves molecule 2 and the anion below the imidazolium ring; the disordered counterpart is longer [3.031(17)] $\AA$ and at $2.84 \AA$ the $\mathrm{H} \cdots \mathrm{F}$ distance is greater than the sum of the van der Waals radii; the associated contact angles are 99 and $92^{\circ}$, respectively. At 0.07 GPa and ambient temperature, the corresponding distances and angles are $2.74(3) \AA 2,112^{\circ}$ and 3.19(3) $\AA, 118^{\circ}$, respectively, and all $\mathrm{H} \cdots \mathrm{F}$ distances are less than the sum of the van der Waals radii. At high pressure, the number of short $\mathrm{H} \cdots \mathrm{F}$ contacts is lower than at low temperature, in line with the lower density, and the environment around the symmetry independent molecules more similar when considering average values, in line with the higher pseudo-symmetry.

All three polymorphs exhibit close contacts that involve not only $\mathrm{H} 2$ but all ring as well as the methyl hydrogen atoms; overall, the more linear contacts are associated with longer C...F distances. The former observation seems to suggest that close contacts between these atoms and anions are not directly responsible for the observed cation conformation in the solid state. On inspection of Table 7.5 and Table 7.6 deposited as Appendix other more indicative trends emerge. 
The GT conformer appears to be stabilised by a contact to the hydrogen atom attached to C7 and no contacts to $\mathrm{C} 8$ or $\mathrm{C} 10$ (for which distances are $>3.7 \AA$ ); the G'T conformer is additionally stabilised to contacts to $\mathrm{C} 10$ (the contact to $\mathrm{H} 8$ observed at $193 \mathrm{~K}$ is long: H8 $\cdots \mathrm{F}$ distance $=2.67 \AA$ and $\mathrm{C} 8 \cdots \mathrm{F}$ distance $=3.662(5) \AA$ ). The difference between the TT, $\mathrm{TG}^{\prime}$ and $\mathrm{TE}^{\prime}$ conformers is not immediately evident but all involve additional contacts to the terminal butyl carbon atom. Differences in the number of contacts involving methyl hydrogen atoms are inconclusive given that their positions are not reliable.

\subsubsection{General discussion}

Our experiments indicate that the $\alpha$-polymorph is very sensitive to temperature changes and should be kept well below the transition temperatures to the $\beta$ - or $\gamma$-phases. The temperature range used for in situ crystallisation followed by crystal growth on a diffractometer by zone melting, e.g. using an Optical Heating and Crystallisation Device (OHCD), is usually quite large and difficult to control: this could partially explain why single-crystal structures reported in the literature correspond to the $\gamma$-phase despite the fact that the crystal was grown at temperatures where the $\alpha$-phase is stable. ${ }^{11}$ The reproducibility of the crystallisation protocols outlined in section 7.3.4 seems to be strongly dependent on a number of factors, including sample volume, water contamination and coexistence of liquid and solid phases. For instance, nucleation of the $\alpha$-phase is most reproducible with sample volumes greater than ca. $200 \mu \mathrm{L}$; similarly, crystallisation of the $\beta$ phase appears to be more reproducible when the sample is contained in a capillary and the heating rates are high. Protocols 3 and 4 outlined in section 7.3.4 are most successful when the whole sample is crystalline: when the liquid and crystalline phases coexist, the $\alpha$ to liquid phase transition can occur at $c a .263 \mathrm{~K}$, without a prior solid-solid phase transition. The presence of water as a contaminant, either as a liquid or as ice, also seems to facilitate the formation of the $\gamma$-form, though this has not been tested systematically. Interestingly, the presence of water also seems to be crucial for the crystallisation of the $\gamma$-phase under high-pressure conditions: preliminary experiments indicate that crystallisation of $\left[\mathrm{bmim}\left[\mathrm{PF}_{6}\right]\right.$ from very diluted aqueous solutions in the 0.4-0.7 GPa pressure range results in the formation of the $\gamma$-form, as confirmed by single-crystal Xray diffraction. In line with other authors, on increasing pressure, we did not observe the crystallisation of the $\gamma$-phase from the pure liquid, suggesting that, based on density differences, the application of modest pressure favours crystallisation of the $\beta$-phase.

The existence of multiple conformers in the liquid state of $[\mathrm{bmim}]\left[\mathrm{PF}_{6}\right]$ is well documented. ${ }^{12,40} \mathrm{~A}$ recent elegant study by Jeon $\mathrm{el}$ al. reported the nanocrystallisation of $[\mathrm{bmim}]\left[\mathrm{PF}_{6}\right]$ at the vapour-liquid interface at $310 \mathrm{~K}$ by grazing-angle synchrotron $\mathrm{X}$-ray diffraction. ${ }^{41}$ The sharp Bragg diffraction peaks observed by the authors, arising from nanocrystallites at the interface and coexisting with the broad diffraction features of the liquid, 
were indexed and a quasi-2D long-range ordered structure was extracted which was similar to that of the $\gamma$-form. Whilst we also observe coexistence of crystalline and liquid states at certain conditions of temperature and pressure, our discrete Bragg reflections collected in transmission geometry are very starkly dominated by the crystalline bulk: hence, the cation and anion disorder were analysed in terms of crystalline disorder. Surface diffraction methods could shed more light into the nature of the crystal-liquid interfaces. It could be conceivable, in particular for the $\beta$-phase, where disorder is more severe, that different cooling rates would result in a different distribution of conformers - however, data collected on different crystals suggest that the differences are not significant, so that the main trends reported are confirmed.

As indicated in the Experimental section, crystals of the $\beta$-phase obtained at high-pressure or low-temperature conditions are characterised by twinning. The $\beta$-phase was found to be stable between ca. 0.07 and 0.4 GPa. As expected, the lowest deviation from perfect monoclinic $C$ symmetry was observed for the high-pressure structure collected at $0.07 \mathrm{GPa}$, i.e. at phase-boundary conditions, and this is also the structure exhibiting the highest degree of pseudosymmetry and perfect domain overlap. Further compression of the single crystal from 0.07 to 0.35 GPa resulted in a unit-cell with a higher degree of deviation from a perfect monoclinic $C$ cell. This is a small pressure range to probe the effects of pressure on disorder; however, our data analysis indicates no significant changes in disorder on increasing pressure.

Whilst the study of conformational flexibility and energetic aspects of the $[\mathrm{bmim}]^{+}$cation in the gas phase are useful, it is clear that an extrapolation of the findings to structures adopted in the solid state is not entirely justified since DFT gas phase calculations on isolated cations ignore the effects of anions and the stabilising energy contributions that arise from crystal packing. It should be noted that whilst Tsuzuki et al. have studied anion effects in their conformational analysis, the $\left[\mathrm{PF}_{6}\right]^{-}$anion was not included in the calculations. Hence, whilst the three most stable conformers in the gas phase are GT $\left(0.02 \mathrm{kcal} \mathrm{mol}^{-1}\right)$, TT $\left(0.00 \mathrm{kcal} \mathrm{mol}^{-1}\right)$ and $\mathrm{G}^{\prime} \mathrm{T}\left(-0.50 \mathrm{kcal} \mathrm{mol}^{-1}\right)$ (geometries optimised at the MP2/6-311G ${ }^{* *}$ level and relative energies estimated at the $\operatorname{CCSD}(\mathrm{T}) / \mathrm{cc}-\mathrm{pVTZ}$ level), the TG' conformer observed for one of the two independent molecules in the $\beta$-phase at 193 $\mathrm{K}$ is only $c a .4 \mathrm{~kJ} \mathrm{~mol}^{-1}$ less stable than the $\mathrm{G}^{\prime} \mathrm{T}$ rotamer and such a small barrier to rotation can be easily overcome by other stabilising intermolecular interactions in the solid state. The phenomenon of conformational polymorphism in the solid state has been well documented in the literature. Nangia summarises this very nicely: "organic molecules with flexible torsions and low-energy conformers have a greater likelihood of exhibiting polymorphism because (1) different conformations lead to new hydrogen bonding and close-packing modes and (2) the trade-off reduces the total energy difference between alternative crystal structures". ${ }^{42}$ These observations are also applicable in the case of the title compound and confirmed by the values of the phase 
transition enthalpies from DSC measuraments. ${ }^{12}$ Whilst it would be interesting to compare the difference in lattice and interaction energies of the three polymorphs by means of computational calculations, the observed disorder would make the analysis more difficult, and such a study is beyond the scope of this work. Despite the widespread conformational flexibility of the alkyl side chain and the large number of structures available in the CSD, there are not many examples of polymorphs reported for ionic liquids, but those reported are conformational polymorphs; the low incidence of polymorphic structures might be partially due to the fact that the polymorphism of these compounds has not been extensively investigated; for examples see refs. ${ }^{5,33,34}$ and ${ }^{43-45}$.

Although the $\gamma$ - and $\alpha$-phases exhibit very similar Raman spectra, their crystal structures are profoundly different. The crystal structures of the three polymorphs are substantially different from one another: hence, X-ray powder diffraction can be an effective tool for rapid phase identification. Two forms were previously identified by Triolo et al. ${ }^{35}$ using wide-angle X-ray scattering (cr-I and cr-II, see Table 7.1). Comparison of simulated patterns (see Figure 7.10 in the Appendix) derived from the single-crystal structures of the three forms with the patterns reported in this previous paper indicates the following: the patterns labelled cr-I (see Figures 8, 9 and 10 of the original paper) correspond to the $\alpha$-phase, whilst the patterns labelled cr-II correspond to the $\gamma$ phase (see Figures 9 and 10 of the original paper). The $\beta$-form was not reported. It is interesting to note that the powder data clearly indicate the occurrence of the $\alpha \rightarrow \gamma$ transition at $250 \mathrm{~K}$, also confirmed by thermal analysis, despite the fact that a similar temperature was reported for the $\alpha \rightarrow$ $\beta$ transition by Endo et al. Our experiments confirm that sample thermal history, impurities and heating/cooling rates are important factors affecting the polymorphic phase transitions in $[\mathrm{bmim}]\left[\mathrm{PF}_{6}\right]$.

\subsection{Conclusions}

The polymorphic behaviour of the ionic liquid $[\mathrm{bmim}]\left[\mathrm{PF}_{6}\right]$ has been studied by Raman spectroscopy, single-crystal X-ray diffraction and optical microscopy. The existence of three polymorphs, $\alpha, \beta$ and $\gamma$, which can be crystallised as a function of low temperature or high pressure, has been confirmed and all three have been structurally characterised. The conformational flexibility of the $[\mathrm{bmim}]^{+}$cation, together with the capability of rotational disorder of the $\left[\mathrm{PF}_{6}\right]^{-}$anion, give rise to different conformations in the solid state not only as a function of crystallisation conditions but also of changes in temperature and pressure to the same phase. The range of cation conformations identified provides a basis by which to validate simulation and modelling data for both fundamental studies and process design. The GT, TT and G'T conformations of the cation in the $\alpha$-, $\beta$ - and $\gamma$-phases, respectively, inferred from previous analysis of Raman stretching frequency, is essentially confirmed; however, the availability for the first time 
of single-crystal X-ray data for all polymorphs reveals a wider range of conformers than previously thought. Across the three phases and under different experimental conditions, a large variation in crystal packing densities and in structural disorder is observed. These variations are likely contributing driving forces for the polymorphic interconversions. Whilst phase transitions do not occur in a single-crystal-to-single-crystal fashion and the molecular packing arrangements of the three forms are very distinct, some continuity in the structural motifs of the building blocks is observed. 


\subsection{References}

(1) Youngs, T. G. A.; Hardacre, C.; Claire Lisa Mullan. Experimental and Theoretical Structure of Ionic Liquids. In Ionic Liquids in Chemical Analysis; Koel, M., Ed.; CRC Press: Durham, NC, USA, 2008; $\mathrm{p} 73$.

(2) Hardacre, C.; Holbrey, J. D.; Nieuwenhuyzen, M.; Youngs, T. G. A. Structure and Solvation in Ionic Liquids. Acc. Chem. Res. 2007, 40 (11), 1146-1155.

(3) Triolo, A.; Mandanici, A.; Russina, O.; Rodriguez-Mora, V.; Cutroni, M.; Hardacre, C.; Nieuwenhuyzen, M.; Bleif, H.-J.; Keller, L.; Ramos, M. A. Thermodynamics, Structure, and Dynamics in Room Temperature Ionic Liquids: The Case of 1-Butyl-3-Methyl Imidazolium Hexafluorophosphate ([bmim][PF6]). J. Phys. Chem. B 2006, 110 (42), 21357-21364.

(4) Allen, F. H. The Cambridge Structural Database: A Quarter of a Million Crystal Structures and Rising. Acta Crystallogr. Sect. B Struct. Sci. 2002, 58, 380-388.

(5) Holbrey, J. D.; Reichert, W. M.; Nieuwenhuyzen, M.; Johnson, S.; Seddon, K. R.; Rogers, R. D.; Johnston, S.; Kenneth, R. Crystal Polymorphism in 1-Butyl-3-Methylimidazolium Halides : Supporting Ionic Liquid Formation by Inhibition of Crystallization. Chem. Commun. 2003, No. 14, 1636-1637.

(6) Bhargava, B. L.; Balasubramanian, S. Insights into the Structure and Dynamics of a RoomTemperature Ionic Liquid: Ab Initio Molecular Dynamics Simulation Studies of 1-N-Butyl3-Methylimidazolium Hexafluorophosphate ([bmim][PF6]) and the [bmim][PF6]-CO2 Mixture. J. Phys. Chem. B 2007, 111, 4477-4487.

(7) Bhargava, B. L.; Balasubramanian, S. Refined Potential Model for Atomistic Simulations of Ionic Liquid [bmim][PF6]. J. Chem. Phys. 2007, 127, 114510.

(8) Del Pópolo, M. G.; Lynden-Bell, R. M.; Kohanoff, J. Ab Initio Molecular Dynamics Simulation of a Room Temperature Ionic Liquid. J. Phys. Chem. B 2005, 109 (12), 5895-5902.

(9) Bühl, M.; Chaumont, A.; Schurhammer, R.; Wipff, G. Ab Initio Molecular Dynamics of Liquid 1,3-Dimethylimidazolium Chloride. J. Phys. Chem. B 2005, 109, 18591-18599.

(10) Dibrov, S. M.; Kochi, J. K. Crystallographic View of Fluidic Structures for RoomTemperature Ionic Liquids: 1-Butyl-3-Methylimidazolium Hexafluorophosphate. Acta Crystallogr. C. 2006, 62 (Pt 1), o19-o21.

(11) Choudhury, A. R.; Winterton, N.; Steiner, A.; Cooper, A. I.; Johnson, K. A. In Situ Crystallization of Low-Melting Ionic Liquids. J. Am. Chem. Soc. 2005, 127 (48), 16792-16793.

(12) Endo, T.; Kato, T.; Tozaki, K.; Nishikawa, K. Phase Behaviors of Room Temperature Ionic Liquid Linked with Cation Conformational Changes: 1-Butyl-3-Methylimidazolium Hexafluorophosphate. J. Phys. Chem. B 2010, 114, 407-411.

(13) Endo, T.; Murata, H.; Imanari, M.; Mizushima, N.; Seki, H.; Nishikawa, K. NMR Study of Cation Dynamics in Three Crystalline States of 1-Butyl-3-Methylimidazolium Hexafluorophosphate Exhibiting Crystal Polymorphism. J. Phys. Chem. B 2012, 116 (12), 3780-3788.

(14) Tsuzuki, S.; Arai, A. A.; Nishikawa, K. Conformational Analysis of 1-Butyl-3Methylimidazolium by $\operatorname{CCSD}(\mathrm{T})$ Level $\mathrm{Ab}$ Initio Calculations: Effects of Neighboring Anions. J. Phys. Chem. B 2008, 112 (26), 7739-7747.

(15) Russina, O.; Fazio, B.; Schmidt, C.; Triolo, A. Structural Organization and Phase Behaviour of 1-Butyl-3-Methylimidazolium Hexafluorophosphate: An High Pressure Raman Spectroscopy Study. Phys. Chem. Chem. Phys. 2011, 13 (25), 12067-12074.

(16) Yoshimura, Y.; Takekiyo, T.; Imai, Y.; Abe, H. High Pressure Phase Behavior of Two Imidazolium-Based Ionic Liquids, [bmim][BF4] and [bmim][PF6]. In Ionic Liquids - Classes 
and Properties; Handy, S., Ed.; InTech Published, 2011; pp 171-186.

(17) Su, L.; Li, M.; Zhu, X.; Wang, Z.; Chen, Z.; Li, F.; Zhou, Q.; Hong, S. In Situ Crystallization of Low-Melting Ionic Liquid [BMIM][PF6] under High Pressure up to 2 GPa. J. Phys. Chem. B 2010, 114 (15), 5061-5065.

(18) Su, L.; Li, L.; Hu, Y.; Yuan, C.; Shao, C.; Hong, S. Phase Transition of [Cn-Mim] [PF6] under High Pressure up to 1.0 GPa. J. Chem. Phys. 2009, 130 (18), 184503.

(19) de Azevedo, R. G.; Esperança, J. M. S. S.; Najdanovic-Visak, V.; Visak, Z. P.; Guedes, H. J. R.; da Ponte, M. N.; Rebelo, L. P. N. Thermophysical and Thermodynamic Properties of 1Butyl-3-Methylimidazolium Tetrafluoroborate and 1-Butyl-3-Methylimidazolium Hexafluorophosphate over an Extended Pressure Range. J. Chem. Eng. Data 2005, 50, $997-$ 1008 .

(20) Zhao, Y.; Liu, X.; Lu, X.; Zhang, S.; Wang, J.; Wang, H.; Gurau, G.; Rogers, R. D.; Su, L.; Li, $\mathrm{H}$. The Behavior of Ionic Liquids Under High Pressure: A Molecular Dynamics Simulation. J. Phys. Chem. B 2012, 116 (35), 10876-10884.

(21) Fabbiani, F. P. A.; Levendis, D. C.; Buth, G.; Kuhs, W. F.; Shanklandd, N.; Sowaa, H. Searching for Novel Crystal Forms by in Situ High-Pressure Crystallisation: The Example of Gabapentin Heptahydrate. CrystEngComm 2010, 12, 2354-2360.

(22) Piermarini, G. J.; Block, S.; Barnett, J. D.; Forman, R. A. Calibration of the Pressure Dependence of the R1 Ruby Fluorescence Line to 195 Kbar. J. Appl. Phys. 1975, 46 (6), 27742780 .

(23) Bruker-Nonius. SAINT Version 7.32A, 2006, Bruker-AXS, Madison, Wisconsin, USA. SAINT version 7.32A, 2006, Bruker-AXS: Madison, Wisconsin, USA.

(24) Dawson, A.; Allan, D. R.; Parsons, S.; Ruf, M. Use of a CCD Diffractometer in Crystal Structure Determinations at High Pressure. J. Appl. Crystallogr. 2004, 37 (3), 410-416.

(25) Sheldrick, G. M. SADABS Version 2008-1, Bruker-AXS, Madison, Wisconsin, USA. SADABS Version 2008-1, 2008, Bruker-AXS: Madison, Wisconsin, USA 2008.

(26) Parsons, S. SHADE, 2004, The University of Edinburgh: Scotland. SHADE, 2004, The University of Edinburgh: Edinburgh, Scotland.

(27) Sheldrick, G. M. A Short History of SHELX. Acta Crystallogr. Sect. A Found. Crystallogr. 2008, $64,112-122$.

(28) Hübschle, C. B.; Sheldrick, G. M.; Dittrich, B. ShelXle: A Qt Graphical User Interface for SHELXL. J. Appl. Crystallogr. 2011, 44, 1281-1284.

(29) Sheldrick, G. M. TWINABS. TWINABS version 2008/2, 2008, Bruker-AXS: Madison, Wisconsin, USA.

(30) Watkin, D. Structure Refinement: Some Background Theory and Practical Strategies. J. Appl. Crystallogr. 2008, 41 (3), 491-522.

(31) Berg, R. W. Raman Spectroscopy, Ab Initio Model Calculations and Conformational Equilibria in Ionic Liquids. In Ionic Liquids in Chemical Analysis; Koel, M., Ed.; CRC Press: Durham, NC, USA, 2008; p 307.

(32) Berg, R. W.; Deetlefs, M.; Seddon, K. R.; Shim, I.; Thompson, J. M. Raman and Ab Initio Studies of Simple and Binary 1-Alkyl-3-Methylimidazolium Ionic Liquids. J. Phys. Chem. B 2005, 109 (40), 19018-19025.

(33) Downard, A.; Earle, M. J.; Hardacre, C.; Mcmath, S. E. J.; Nieuwenhuyzen, M.; Teat, S. J. Structural Studies of Crystalline 1-Alkyl-3-Methylimidazolium Chloride Salts. Chem. Mater. 2004, 16, 43-48.

(34) Gordon, C. M.; Holbrey, J. D.; Kennedy, A. R.; Seddon, K. R. Ionic Liquid Crystals: 
Hexafluorophosphate Salts. J. Mater. Chem. 1998, 8, 2627-2636.

(35) Triolo, A.; Russina, O.; Hardacre, C.; Nieuwenhuyzen, M.; Gonzalez, M. A.; Grimm, H. Relaxation Processes in Room Temperature Ionic Liquids: The Case of 1-Butyl-3-Methyl Imidazolium Hexafluorophosphate. J. Phys. Chem. B 2005, 109 (46), 22061-22066.

(36) Moura Ramos, J. J.; Afonso, C. A. M.; Branco, L. C. Glass Transition Relaxation and Fragility in Two Room Temperature Ionic Liquids. J. Therm. Anal. Calorim. 2003, 71, 659-666.

(37) Macrae, C. F.; Bruno, I. J.; Chisholm, J. a.; Edgington, P. R.; McCabe, P.; Pidcock, E.; Rodriguez-Monge, L.; Taylor, R.; Van De Streek, J.; Wood, P. a. Mercury CSD 2.0 - New Features for the Visualization and Investigation of Crystal Structures. J. Appl. Crystallogr. 2008, 41 (2), 466-470.

(38) Hardacre, C.; Holbrey, J. D.; McMath, S. E. J.; Bowron, D. T.; Soper, A. K. Structure of Molten 1,3-Dimethylimidazolium Chloride Using Neutron Diffraction. J. Chem. Phys. 2003, 118 (1), 273.

(39) Tsuzuki, S.; Tokuda, H.; Mikami, M. Theoretical Analysis of the Hydrogen Bond of Imidazolium C(2)-H with Anions. Phys. Chem. Chem. Phys. 2007, 9 (34), 4780-4784.

(40) Macchiagodena, M.; Gontrani, L.; Ramondo, F.; Triolo, A.; Caminiti, R. Liquid Structure of 1-Alkyl-3-Methylimidazolium-Hexafluorophosphates by Wide Angle X-Ray and Neutron Scattering and Molecular Dynamics. J. Chem. Phys. 2011, 134 (11), 114521.

(41) Jeon, Y.; Vaknin, D.; Bu, W.; Sung, J.; Ouchi, Y.; Sung, W.; Kim, D. Surface Nanocrystallization of an Ionic Liquid. Phys. Rev. Lett. 2012, 108 (5), 055502.

(42) Nangia, A. Conformational Polymorphism in Organic Crystals. Acc. Chem. Res. 2008, 41 (5), 595-604.

(43) Bäcker, J.; Mihm, S.; Mallick, B.; Yang, M.; Meyer, G.; Mudring, A. V. Crystalline and Liquid Crystalline Organic-Inorganic Hybrid Salts with Cation-Sensitized Hexanuclear Molybdenum Cluster Complex Anion Luminescence. Eur. J. Inorg. Chem. 2011, No. 26, 40894095 .

(44) Kawahata, M.; Endo, T.; Seki, H.; Nishikawa, K.; Yamaguchi, K. Polymorphic Properties of Ionic Liquid of 1-Isopropyl-3-Methylimidazolium Bromide. Chem. Lett. 2009, 38 (12), 11361139 .

(45) Paulechka, Y. U.; Kabo, G. J.; Blokhin, A. V.; Shaplov, A. S.; Lozinskaya, E. I.; Golovanov, D. G.; Lyssenko, K. A.; Korlyukov, A. A.; Vygodskii, Y. S. IR and X-Ray Study of Polymorphism in 1-Alkyl-3-Methylimidazolium Bis(trifluoromethanesulfonyl)imides. J. Phys. Chem. B 2009, 113 (28), 9538-9546.

(46) Spek, A. L. Structure Validation in Chemical Crystallography. Acta Crystallogr. Sect. D Biol. Crystallogr. 2009, 65, 148-155. 


\subsection{Appendix}

Table 7.4. Geometric parameters describing the planar or nearly planar centrosymmetric and pseudocentrosymmetric dimeric arrangements of the cations in the three polymorphs of [bmim][PF 6 .

\begin{tabular}{|c|c|c|c|c|c|}
\hline & & $\begin{array}{c}\text { Centroid-centroid } \\
\text { distance / }\end{array}$ & $\begin{array}{c}\text { Dihedral angle } \\
\text { between ring } \\
\text { planes/ }{ }^{\circ}\end{array}$ & $\begin{array}{c}\text { Interplanar c } \\
\text { distance / ̊ }\end{array}$ & Offset / Å \\
\hline \multirow{2}{*}{$\alpha$-phase } & "open" dimer $\mathbf{1 0 0} \mathrm{K}$ & $5.0554(9)^{\mathrm{a}}$ & 0 & $3.5298(7)$ & 3.619 \\
\hline & "open" dimer $193 \mathrm{~K}$ & $5.0668(14)$ & 0 & $3.6224(11)$ & 3.543 \\
\hline \multirow{6}{*}{$\beta$-phase } & $\begin{array}{l}\text { "open" dimer } \\
193 \text { K Cg1 Cg1 b }\end{array}$ & $4.818(5)$ & 0 & $3.715(4)$ & 3.068 \\
\hline & $\begin{array}{l}\text { "closed" dimer } \\
193 \text { K Cg1 Cg2 }\end{array}$ & $5.950(5)$ & $6.2(5)$ & $5.452(4)$ & - \\
\hline & $\begin{array}{l}\text { "open" dimer } \\
193 \text { K Cg2 Cg2 }\end{array}$ & $4.490(5)$ & 0 & $3.832(4)$ & 2.342 \\
\hline & $\begin{array}{c}\text { "open" dimer } \\
\text { 0.07 GPa Cg1 Cg1 }\end{array}$ & $4.762(12)$ & 0 & $3.877(10)$ & 2.765 \\
\hline & $\begin{array}{c}\text { "closed" dimer } \\
\text { 0.07 GPa Cg1 Cg2 }\end{array}$ & $6.082(12)$ & $3.9(13)$ & $5.542(10)$ & - \\
\hline & $\begin{array}{c}\text { "open" dimer } \\
\text { 0.07 GPa Cg2 Cg2 }\end{array}$ & $4.732(10)$ & 0 & $3.837(8)$ & 2.770 \\
\hline \multirow{4}{*}{$\gamma$-phase } & "open" dimer $193 \mathrm{~K}$ & $4.865(6)$ & 0 & $3.4856(9)$ & 3.394 \\
\hline & "closed dimer $193 \mathrm{~K}$ & $6.133(7)$ & 0 & $4.3688(9)$ & 4.304 \\
\hline & "open" dimer $263 \mathrm{~K}$ & $4.857(2)$ & 0 & $3.5357(15)$ & 3.330 \\
\hline & "closed dimer $263 \mathrm{~K}$ & $6.152(2)$ & 0 & $4.4425(15)$ & 4.255 \\
\hline
\end{tabular}

a All standard deviations were calculated with the program PLATON10. Atoms N1, C2, N3, C4, C5 were chosen to define a ring, the derived centroid and the least-squares plane. ${ }^{b}$ For the $\beta$-phase the aforementioned atoms define Cg1. Atoms N11, C12, N13, C14, C15 define Cg2. c The interplanar distance is defined as the perpendicular distance between the centroid of one ring onto the second ring plane. ${ }^{d}$ The offset is defined as the distance between the centroid of one ring and the perpendicular projection of the centroid of the second ring onto the first ring plane. 
Table 7.5. Coordination numbers for the cation and anion, as well as short $H \cdots F$ contacts in the three polymorphs of [bmim][PF 6 .

\begin{tabular}{|c|c|c|c|c|c|c|}
\hline & & $\begin{array}{l}\text { Cation } \\
\text { C. N. }{ }^{a}\end{array}$ & $\begin{array}{l}\text { Anion } \\
\text { C. } \mathrm{N} \text {. }\end{array}$ & $\begin{array}{c}\mathrm{H} \cdots \mathrm{F} \text { contacts }< \\
\text { sum vdW }\end{array}$ & & $\begin{array}{c}\text { Total number of } \\
\text { H...F contacts }< \\
\text { sum vdW }\end{array}$ \\
\hline \multirow{2}{*}{$\alpha$-phase } & $100 \mathrm{~K}$ & $\begin{array}{l}7 \text { Anions } \\
4 \text { Cations }\end{array}$ & 7 Cations & $\begin{array}{l}\text { Imid. b } \\
\text { Me-imid. } \\
\text { Butyl }\end{array}$ & $\begin{array}{c}\mathrm{H} 2, \mathrm{H} 4, \mathrm{H} 5 \\
\mathrm{H} 6 \mathrm{~B}, \mathrm{H} 6 \mathrm{C} \\
\mathrm{H} 7 \mathrm{~A}\end{array}$ & 8 \\
\hline & $193 \mathrm{~K}$ & $\begin{array}{l}7 \text { Anions } \\
6 \text { Cations }\end{array}$ & 7 Cations & $\begin{array}{l}\text { Imid. } \\
\text { Me-imid. } \\
\text { Butyl }\end{array}$ & $\begin{array}{c}\mathrm{H} 2, \mathrm{H} 4, \mathrm{H} 5 \\
\mathrm{H} 6 \mathrm{~B}, \mathrm{H} 6 \mathrm{C} \\
\mathrm{H} 7 \mathrm{~A}, \mathrm{H} 7 \mathrm{C}\end{array}$ & 5 \\
\hline \multirow{4}{*}{$\beta$-phase } & $\begin{array}{l}193 \mathrm{~K} \\
\text { Mol } 1\end{array}$ & $\begin{array}{l}7 \text { Anions } \\
3 \text { Cations }\end{array}$ & $\begin{array}{l}1 \text { Anion } \\
8 \text { Cations }\end{array}$ & $\begin{array}{l}\text { Imid. } \\
\text { Me-imid. } \\
\text { Butyl }\end{array}$ & $\begin{array}{c}\mathrm{H} 2, \mathrm{H} 4, \mathrm{H} 5 \\
\mathrm{H} 6 \mathrm{~A}, \mathrm{H} 6 \mathrm{~B} \\
\text { H7A, H7B, H8A, } \\
\text { H8B }\end{array}$ & 8 \\
\hline & $\begin{array}{l}193 \mathrm{~K} \\
\mathrm{Mol} 2\end{array}$ & $\begin{array}{l}6 \text { Anions } \\
2 \text { Cations }\end{array}$ & $\begin{array}{l}1 \text { Anion } \\
8 \text { Cations }\end{array}$ & $\begin{array}{l}\text { Imid. } \\
\text { Me-imid. } \\
\text { Butyl }\end{array}$ & $\begin{array}{c}\text { H12, H14, H15 } \\
\text { H16A, H16B, } \\
\text { H16C } \\
\text { H17B, H18A, } \\
\text { H20A }\end{array}$ & 7 \\
\hline & $0.07 \mathrm{GPa} \mathrm{Mol} 1$ & $\begin{array}{l}8 \text { Anions } \\
4 \text { Cations }\end{array}$ & 8 Cations & $\begin{array}{l}\text { Imid. } \\
\text { Me-imid. } \\
\text { Butyl }\end{array}$ & $\begin{array}{c}\mathrm{H} 2, \mathrm{H} 4, \mathrm{H} 5 \\
\text { H6A, H6B } \\
\text { H7A, H7B, H8A, } \\
\text { H8C }\end{array}$ & 6 \\
\hline & $0.07 \mathrm{GPa} \mathrm{Mol} 2$ & $\begin{array}{l}7 \text { Anions } \\
4 \text { Cations }\end{array}$ & 8 Cations & $\begin{array}{l}\text { Imid. } \\
\text { Me-imid. } \\
\text { Butyl }\end{array}$ & $\begin{array}{l}\text { H12, H14, H15 } \\
\text { H16A, H16C, } \\
\text { H17A, H17B, } \\
\text { H18A, H20A }\end{array}$ & 6 \\
\hline \multirow[b]{2}{*}{$\gamma$-phase } & $193 \mathrm{~K}$ & $\begin{array}{l}8 \text { Anions } \\
4 \text { Cations }\end{array}$ & 8 Cations & $\begin{array}{l}\text { Imid. } \\
\text { Me-imid. } \\
\text { Butyl }\end{array}$ & $\begin{array}{c}\text { H2, H4, H5 } \\
\text { H6A, H6B } \\
\text { H7B, H8A, } \\
\text { H10A, H10C }\end{array}$ & 11 \\
\hline & $263 \mathrm{~K}$ & $\begin{array}{l}8 \text { Anions } \\
5 \text { Cations }\end{array}$ & 8 Cations & $\begin{array}{l}\text { Imid. } \\
\text { Me-imid. } \\
\text { Butyl }\end{array}$ & $\begin{array}{c}\text { H2, H4, H5 } \\
\text { H6A, } \\
\text { H7B, H7D, } \\
\text { H10A, H10C, } \\
\text { H10D }\end{array}$ & 9 \\
\hline
\end{tabular}

radii $+0.2 \AA$. Contacts were identified using the programs PLATON ${ }^{46}$ and MERCURY. ${ }^{37} \mathrm{vdW}$ radius for $\mathrm{H}=1.20 \AA$, vdW radius for $\mathrm{F}=1.47 \AA$ A. For the determination of C.N. all disorder components were considered. ${ }^{\mathrm{b}} \mathrm{H}$ atoms are grouped according their chemical environment: Imidazolium (Imid.), Methyl-imidazolium (Me-imid.) and butyl (Butyl). c Total number of contacts: contacts involving disordered atoms were weighted according to site occupancies and rounded to the nearest integer, see Table 7.6 for details. 
Table 7.6. Short contacts with $H$...F distances < sum of $v d W$ radii. $v d W$ radius for $H=1.20 \AA$, vdW radius for $\mathbf{F}=1.47 \AA$. The programs MERCURY ${ }^{37}$ and PLATON $^{46}$ were used to check these values.

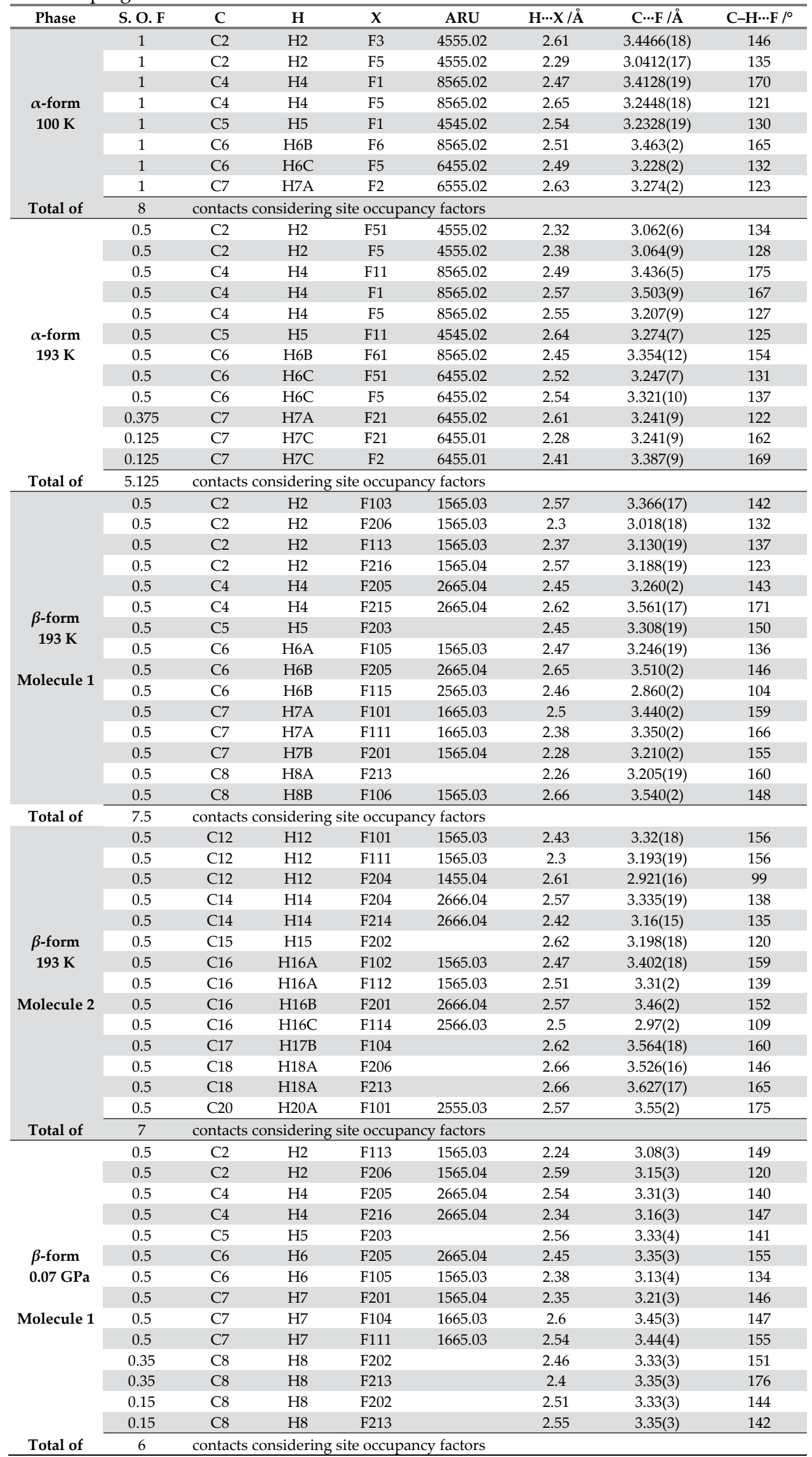




\begin{tabular}{|c|c|c|c|c|c|c|c|c|}
\hline \multirow{7}{*}{$\begin{array}{l}\beta \text {-form } \\
0.07 \mathrm{GPa}\end{array}$} & 0.5 & $\mathrm{C} 12$ & H12 & F101 & 1565.03 & 2.51 & $3.26(3)$ & 138 \\
\hline & 0.5 & $\mathrm{C} 12$ & H12 & F111 & 1565.03 & 2.44 & $3.20(3)$ & 139 \\
\hline & 0.5 & $\mathrm{C} 12$ & H12 & F204 & 1455.04 & 2.24 & $2.74(3)$ & 112 \\
\hline & 0.5 & $\mathrm{C} 12$ & H12 & F214 & 1455.04 & 2.64 & $3.19(3)$ & 118 \\
\hline & 0.5 & $\mathrm{C} 14$ & H14 & F201 & 2666.04 & 2.62 & $3.43(3)$ & 147 \\
\hline & 0.5 & C14 & H14 & F214 & 2666.04 & 2.56 & $3.37(3)$ & 146 \\
\hline & 0.5 & $\mathrm{C} 15$ & H15 & F202 & & 2.62 & $3.29(3)$ & 129 \\
\hline \multirow{6}{*}{ Molecule 2} & 0.5 & $\mathrm{C} 16$ & H16 & F102 & 1565.03 & 2.66 & $3.42(4)$ & 136 \\
\hline & 0.5 & C16 & H16 & F114 & 2566.03 & 2.59 & $3.04(3)$ & 109 \\
\hline & 0.5 & $\mathrm{C} 17$ & H17 & F215 & 1455.04 & 2.38 & $3.29(4)$ & 156 \\
\hline & 0.5 & $\mathrm{C} 17$ & H17 & F103 & & 2.61 & $3.49(4)$ & 150 \\
\hline & 0.5 & $\mathrm{C} 18$ & H18 & F213 & & 2.43 & $3.38(4)$ & 170 \\
\hline & 0.35 & C20 & $\mathrm{H} 20$ & F101 & & 2.65 & $3.57(4)$ & 162 \\
\hline Total of & 6.35 & \multicolumn{7}{|c|}{ contacts considering site occupancy factors } \\
\hline \multirow{11}{*}{$\begin{array}{c}\gamma \text {-form } \\
193 \mathrm{~K}\end{array}$} & 1 & $\mathrm{C} 2$ & $\mathrm{H} 2$ & F5 & & 2.35 & $3.233(5)$ & 152 \\
\hline & 1 & $\mathrm{C} 2$ & $\mathrm{H} 2$ & F3 & 2766.02 & 2.6 & $3.192(4)$ & 120 \\
\hline & 1 & $\mathrm{C} 4$ & $\mathrm{H} 4$ & F2 & 2756.02 & 2.45 & $3.38(5)$ & 164 \\
\hline & 1 & C5 & H5 & F2 & 1656.02 & 2.67 & $3.563(5)$ & 155 \\
\hline & 1 & C5 & H5 & F3 & 1656.02 & 2.59 & $3.418(4)$ & 145 \\
\hline & 1 & C6 & H6A & $\mathrm{F} 4$ & 2756.02 & 2.49 & $3.342(5)$ & 148 \\
\hline & 1 & C6 & H6B & $\mathrm{F} 1$ & 1655.02 & 2.67 & $3.382(5)$ & 131 \\
\hline & 1 & $\mathrm{C} 7$ & H7B & F6 & 1656.02 & 2.53 & $3.309(4)$ & 138 \\
\hline & 1 & $\mathrm{C} 8$ & H8A & F5 & 2767.02 & 2.67 & $3.622(5)$ & 175 \\
\hline & 1 & $\mathrm{C} 10$ & H10A & F3 & 1556.02 & 2.58 & $3.503(5)$ & 162 \\
\hline & 1 & $\mathrm{C} 10$ & $\mathrm{H} 10 \mathrm{C}$ & $\mathrm{F} 2$ & 2656.02 & 2.66 & $3.553(5)$ & 155 \\
\hline Total of & 11 & \multicolumn{7}{|c|}{ contacts considering site occupancy factors } \\
\hline \multirow{11}{*}{$\begin{array}{c}\gamma \text {-form } \\
263 \mathrm{~K}\end{array}$} & 1 & $\mathrm{C} 2$ & $\mathrm{H} 2$ & F5 & & 2.41 & $3.255(5)$ & 152 \\
\hline & 1 & $\mathrm{C} 2$ & $\mathrm{H} 2$ & F3 & 2766.02 & 2.66 & $3.263(5)$ & 123 \\
\hline & 1 & $\mathrm{C} 4$ & $\mathrm{H} 4$ & F2 & 2756.02 & 2.51 & $3.419(5)$ & 165 \\
\hline & 1 & C5 & H5 & F3 & 1656.02 & 2.65 & $3.461(4)$ & 147 \\
\hline & 1 & C6 & H6A & $\mathrm{F} 4$ & 2756.02 & 2.56 & $3.42(5)$ & 149 \\
\hline & 0.92 & $\mathrm{C} 7$ & H7B & F6 & 1656.02 & 2.57 & $3.336(4)$ & 136 \\
\hline & 0.08 & C7 & H7D & F6 & 1656.02 & 2.37 & $3.336(4)$ & 173 \\
\hline & 0.92 & C8A & H8A & F5 & 2767.02 & 2.66 & $3.632(5)$ & 176 \\
\hline & 0.92 & $\mathrm{C} 10$ & $\mathrm{H} 10 \mathrm{~A}$ & F3 & 1556.02 & 2.6 & $3.513(6)$ & 159 \\
\hline & 0.92 & $\mathrm{C} 10$ & $\mathrm{H} 10 \mathrm{C}$ & F2 & 2656.02 & 2.66 & $3.592(5)$ & 165 \\
\hline & 0.08 & $\mathrm{C} 10$ & H10D & F3 & 2765.02 & 2.66 & $3.513(6)$ & 149 \\
\hline Total of & 8.84 & \multicolumn{7}{|c|}{ contacts considering site occupancy factors } \\
\hline
\end{tabular}




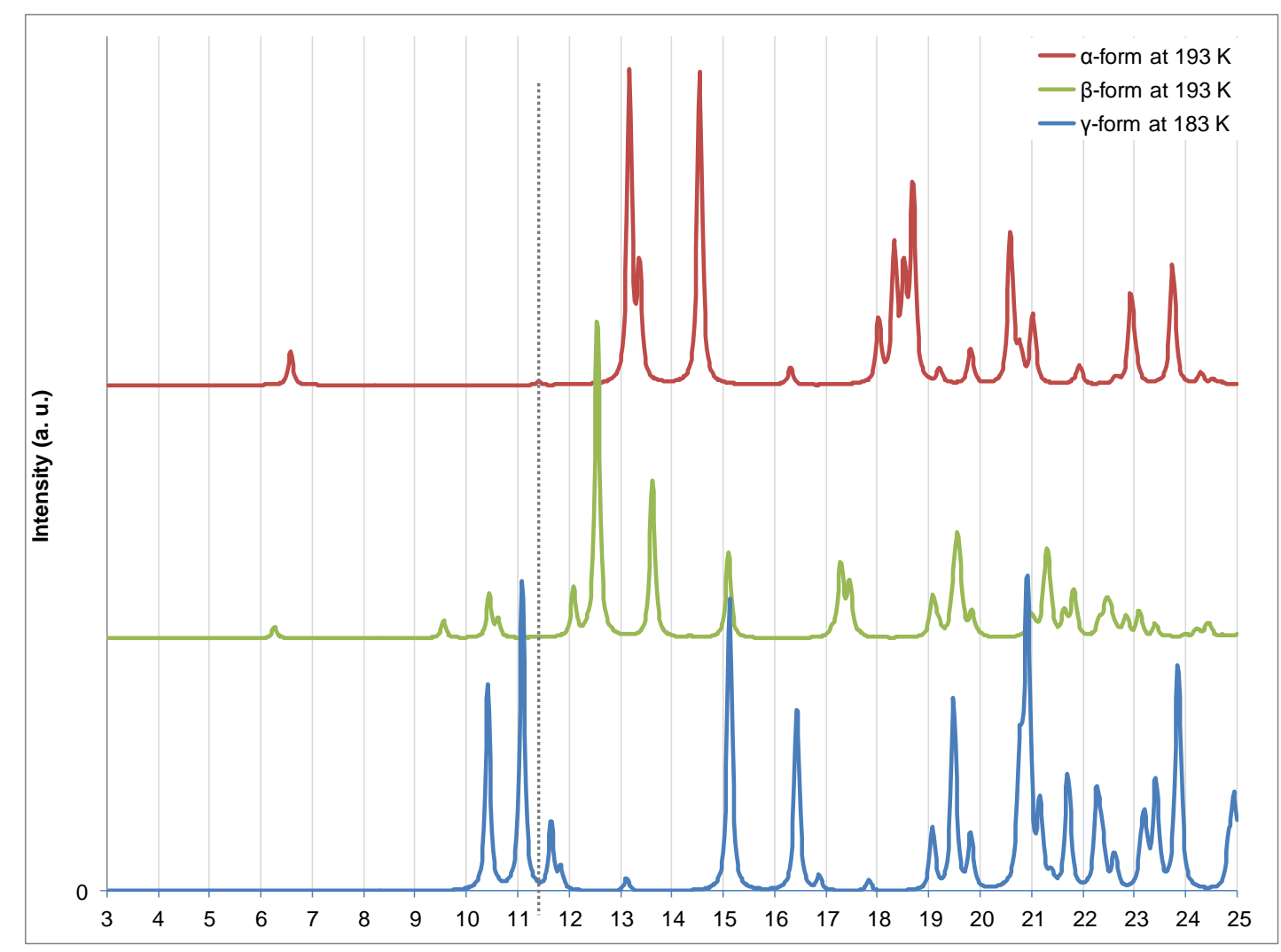

Figure 7.10. Powder patterns simulated using the experimentally-determined single-crystal structures as input. For the $\gamma$-form, MAZXOB01 was used. Solid lines are used as a guide to the eye to facilitate comparison with Figures 8, 9 and 10 in the paper for Triolo et al. ${ }^{3}$ The dotted line at $2 \theta=11.3^{\circ}\left(\mathrm{Q}=0.8 \AA^{-1}\right)$ is characteristic for the $\alpha$-form. The program Mercury ${ }^{37}$ was used to generate the simulated patterns. Input parameters were as following: $\lambda=1.54056 \AA$; $2 \theta$ range: $3-25^{\circ}, 2$ Theta Step: $0.02^{\circ}$. FWHM Peak shape: 0.1 . 


\section{Chapter 8. Structural behaviour of long-chain imidazolium-based ionic liquid [C10mim]Cl-water mixtures}

\subsection{Synopsis}

The structural behaviour of the long-chain imidazolium-based ionic liquid $\left[\mathrm{C}_{10 \mathrm{mim}}\right] \mathrm{Cl}-$ water mixtures has been investigated in detail by low-temperature and high-pressure crystallisation methods and the solid forms fully characterised by single-crystal X-ray diffraction. Form I, a monohydrate, crystallises from solutions containing $6-8 \%(\mathrm{w} / \mathrm{w})$ water; its structure exhibits bilayers, in which the cations are alternating from layer to layer to create hydrophobic and hydrophilic regions. Form II, which is a $Z^{\prime}>1$ structure, incorporates a variable amount of water content (from pseudo hemihydrate to pseudo monohydrate) depending on sample treatment and environment, crystallises from solutions containing $<6 \%(\mathrm{w} / \mathrm{w})$ water, exhibits a double bilayered structure characterised by ordered and disordered regions. Form III is a trihydrate that has been exclusively obtained under high-pressure conditions above $0.55 \mathrm{GPa}$ from solutions containing > $80 \%(\mathrm{w} / \mathrm{w})$ water. This structure is closely related to that of form I. While homeotypic and isotypic structures of forms I and II have been previously reported, form III represents, to the best of our knowledge, the only example of a highly hydrated long-chain imidazolium-based ionic liquid isolated in the solid state. The formation of mesophases in the title compound has also been investigated by polarized optical microscopy and the results correlated with previous liquidcrystal studies on related compounds.

\subsection{Introduction}

Although the attention towards practical applications of imidazolium-based ILs is still increasing, much fundamental work remains to be done on understanding their phase diagram. Liquid at room temperature, ILs need external factors to crystallise such as temperature, pressure, the addition of solvents and sometimes impurity. There are several articles in the literature describing some aspects of the solid-state behaviour of [ $\left.\mathrm{C}_{\mathrm{nmim}}\right][\mathrm{X}](\mathrm{n} \geq 12, \mathrm{X} \equiv$ halide anion) with structures obtained using conventional crystallisation methods of slow and fast evaporation of solvent mixtures at ambient and high temperature. ${ }^{1-8}$ Interestingly, there are no anhydrous X-ray structures reported in the Cambridge Structural Database ${ }^{9}$ (CSD) for these ILs. In addition, for a given halide and chain length, there are no anhydrous as well as hydrate structures reported for shorter-chain counterparts. ${ }^{10-12}$

Imidazolium-based ILs have also attracted interest for their ability to form liquidcrystalline ionogels; one of the earliest observations on their liquid crystalline behaviour dates from 1996.13 Subsequently, some 1-alkyl-3-methylimidazolium-based ILs [Cnmim][A] ( $\mathrm{n} \geq 10 \mathrm{~A} \equiv$ anion) 
were described as either thermotropic liquid crystals, ${ }^{14}$ or lyotropic liquid crystals. ${ }^{15-19}$ Furthermore, it is not uncommon to see mesophase formation for shorter alkyl chain imidazoliumbased halides. ${ }^{20-22}$

Following our work on 1-butyl-3-methyimidazolium hexafluorophosphate, ${ }^{23}$ in which the rich phase and crystallisation diagram of the compound was explored and elucidated, in this article we report and compare the results of low-temperature and high-pressure crystallisation experiments of 1-decyl-3-methylimidazolium chloride, [ $\mathrm{C}_{10 \mathrm{mim}} \mathrm{Cl}$ (Figure 8.1), as function of variable water content, as revealed by hot-stage microscopy and single-crystal X-ray diffraction.

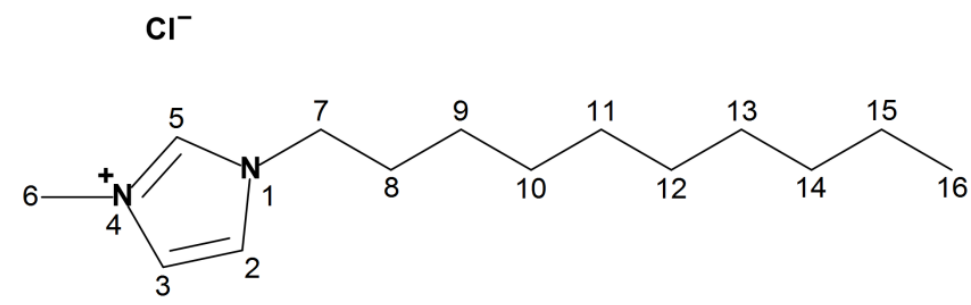

Figure 8.1. Chemical diagram of $\left[\mathrm{C}_{10 \mathrm{mim}}\right] \mathrm{Cl}$ with atom numbering scheme.

\subsection{Experimental}

A combination of Roman numerals and alphabetical letters are the structure identifiers used throughout this article. The same Roman numeral is given to structures with similar unit-cell parameters, the same space group and crystal packing. The letter following the Roman numeral indicates variation in water content and/or position in different crystal structures.

\subsubsection{Material}

Please refer to the corresponding sections below detailing the treatment of $\left[\mathrm{C}_{10 \mathrm{mim}}\right] \mathrm{Cl}$ [Aldrich, 95.5\% purity, $\mathrm{H}_{2} \mathrm{O} \leq 2 \%(w / w)$ ] prior to use.

\subsubsection{Low-temperature crystallisation technique}

All low-temperature experiments were conducted using a Linkam THMS600 heating and freezing stage. The stage enables temperature control within a precision of $0.1 \mathrm{~K}$ and sample viewing through a quartz glass window. The experimental setup allows for a protective $\mathrm{N}_{2}$ gas flow at the sample for operating temperatures below $328 \mathrm{~K}$.

\subsubsection{Form I (LT): crystallisation of [C10 $\mathrm{mim}] \mathrm{Cl}$ monohydrate}

A drop of an aqueous [ $\left.\mathrm{C}_{10 \mathrm{mim}}\right] \mathrm{Cl}$ solution (solutions in the range $6-8 \%(\mathrm{w} / \mathrm{w}) \mathrm{H}_{2} \mathrm{O}$ were tried) was introduced into the sample chamber of the Linkam stage. A slow cooling rate of 0.3 $\mathrm{K} / \mathrm{min}$ starting from room temperature promoted the crystallisation of plate-like crystals in a temperature range between 269 and $266 \mathrm{~K}$. The crystals melt at $c a .307 \mathrm{~K}$. A single crystal was grown by cycling the temperature between 307 and $304 \mathrm{~K}$. In all cooling cycles, a liquid crystalline 
phase very sensitive to temperature changes was observed (Figure 8.2). The behaviour of this lyotropic phase is highly complex and its presence hinders the growth of another solid phase, corresponding to form II, see below for details. Further cooling of a drop of [ $\left.\mathrm{C}_{10 \mathrm{mim}}\right] \mathrm{Cl}$ below $253 \mathrm{~K}$ promotes the crystallisation of a second solid phase from the remaining liquid part (form II), independent of the cooling rate. Both solid phases can coexist. In some experiments, the coexistence of form I form II and the liquid crystalline phase was observed. A single-crystal of form I was measured at $263 \mathrm{~K}$ by means of $\mathrm{X}$-ray diffraction.
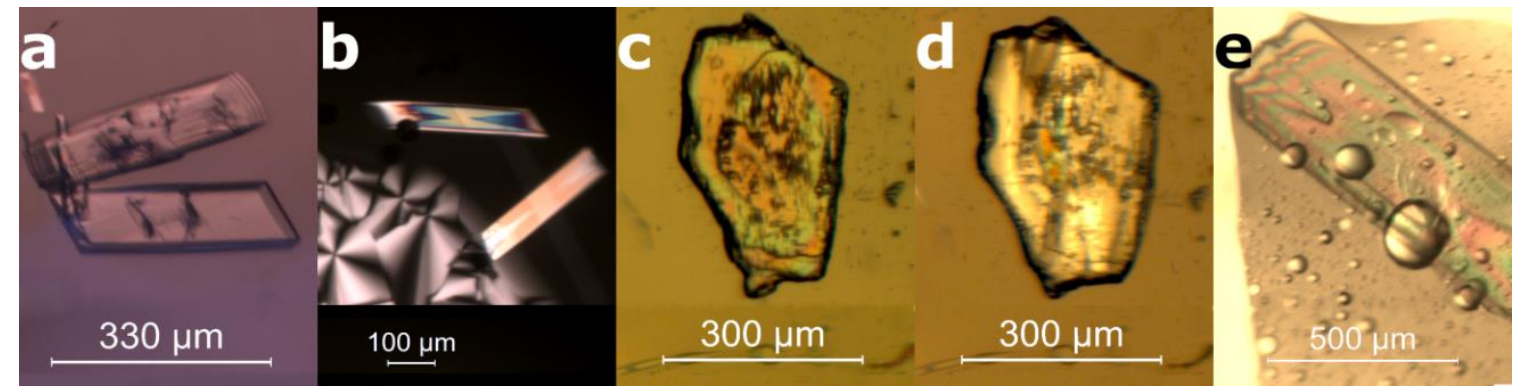

Figure 8.2. Optical microscopy images of the low-temperature crystal growth of forms I and II-a. (a) Form I at $298 \mathrm{~K}$; (b) Form II-a coexisting with the lyotropic phase at $305 \mathrm{~K}$; (c) Fragment of a single crystal of form II-a at $295 \mathrm{~K}$; (d) The single crystal in (c) transformed to the lyotropic phase after 16 hours at ambient conditions;

(e) A single crystal of form II-a under a drop of immersion oil coexisting with the liquid state (droplets).

\subsubsection{Forms II-a/II-b: crystallisation of [C $10 \mathrm{mim}] \mathrm{Cl}$ pseudo-monohydrate}

In addition to the protocol outlined above, form II was also obtained according to the following crystallisation route. A large drop, $1 \mathrm{~mm}$ in diameter, of an aqueous [ $\left.\mathrm{C}_{10 \mathrm{mim}}\right] \mathrm{Cl}$ solution, as prepared for crystallisation of form I, was introduced into the sample chamber of the Linkam stage. In a first step, the drop was heated for 5 minutes at $433 \mathrm{~K}$; the drop was then enclosed with crystal mounting oil (perfluoropolyether) and the system heated a second time for 5 minutes at 473

$K$. These steps served the purpose of eliminating any excess water, as observed by microscopy, that favours the formation of liquid crystals and that might impede the crystallisation of any other form. Unlike form I, form II-a crystallises when fast cooling rates are applied. Cooling to $213 \mathrm{~K}$ using a cooling rate between 50 and $90 \mathrm{~K} / \mathrm{min}$ leads to the crystallisation of a cluster of needles. The phase transition was observed at $253 \mathrm{~K}$; however, the transition temperature depends on the sample volume, water content and cooling rate with the latter having most influence. A single crystal (Figure 8.2) was obtained by cycling the temperature between 305 and $308 \mathrm{~K}$. To preserve crystal quality, the single crystal was separated from the liquid, immersed into mounting oil at ambient condition and subsequently transferred to the diffractometer at $200 \mathrm{~K}$. The same crystallisation procedure was adopted for obtaining form II-b. As described for form I, formation of a liquid crystalline phase between 308 and $305 \mathrm{~K}$ was also observed here. 


\subsubsection{High-pressure crystallisation techniques}

Modified Merrill-Bassett-type ${ }^{24}$ diamond-anvil cells (DACs) were used for the highpressure experiments. ${ }^{25} 200 \mu \mathrm{m}$ Inconel 718 gaskets preindented to $c a .150 \mu \mathrm{m}$ and with a sample chamber $300 \mu \mathrm{m}$ in diameter were used for the experiments. All sample loadings took place at ambient-temperature conditions. Pressure inside the DAC was monitored by the ruby fluorescence method ${ }^{26}$ using an in-house built kit that has an accuracy of $0.05 \mathrm{GPa}$.

\subsubsection{Form I (HP): crystallisation of [ $\left.\mathrm{C}_{10} \mathrm{mim}\right] \mathrm{Cl}$ monohydrate at $0.2 \mathrm{GPa}$}

Subsequent to loading and sealing of the DAC with an aqueous [ $\mathrm{C}_{10 \mathrm{mim}} \mathrm{Cl}$ solution as used for the low-temperature experiments, a liquid crystal phase was observed on increasing pressure just above ambient (Figure 8.3). This phase persists up to $0.3 \mathrm{GPa}$; above this pressure the sample undergoes a phase transition to a crystalline powder. A single crystal was grown by cycling both pressure and temperature. While the crystal was growing the pressure inside the DAC dropped to ambient pressure indicating the crystal is considerably denser than the liquid. Crystal growth necessitates careful monitoring because on one hand when the pressure reaches atmospheric pressures the crystal comes in contact with air humidity, inducing a solid to gel transition, and on the other hand fast pressurisation promotes twinning. The single crystal (Figure 8.3) was measured by means of in situ X-ray diffraction at 0.2 GPa.

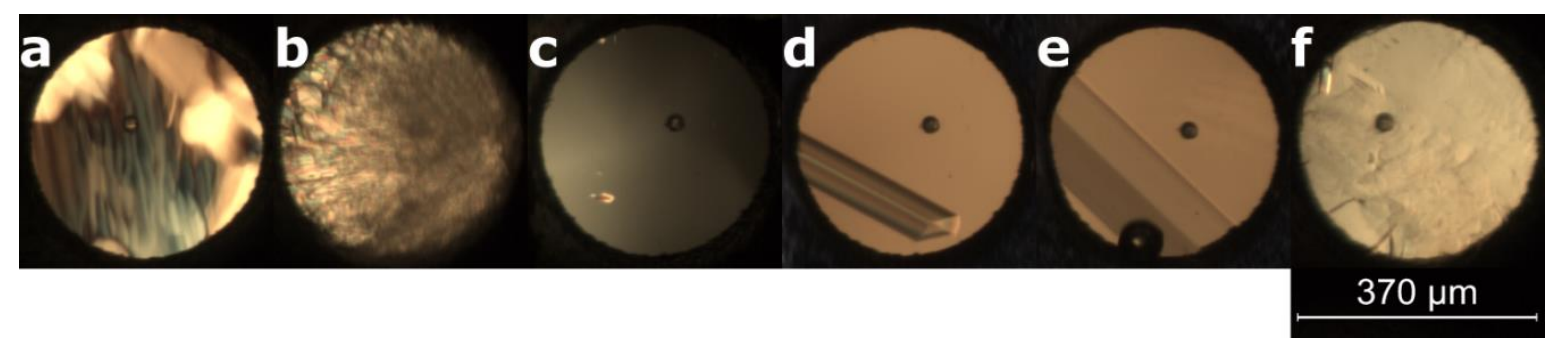

Figure 8.3. Optical microscopy images of the high-pressure growth of form I in a DAC. (a) Lyotropic phase at $0.23 \mathrm{GPa}$; (b) Crystalline powder at $0.35 \mathrm{GPa}$; (c) Single crystal growing after pressure and temperature cycling; (d) Single crystal growing at ca. $0.2 \mathrm{GPa}$; (e) Pressure dropped to atmospheric pressure, as indicated by the presence of an air bubble; (f) Final stage - the single crystal fills the gasket hole at $0.2 \mathrm{GPa}$.

\subsubsection{Form II-c: crystallisation of [ $\left.\mathrm{C}_{10 \mathrm{mim}}\right] \mathrm{Cl}$ hemihydrate at $0.05 \mathrm{GPa}$}

[C $\mathrm{C}_{10 \mathrm{mim}} \mathrm{Cl}$ was dried for 3 hours at $333 \mathrm{~K}$ under moderate $(0.21 \mathrm{mbar})$ vacuum to reduce the water content and a small drop was transferred into a DAC at ambient conditions. Needleshaped crystals grew immediately after closing the DAC at ca. 0.05 GPa (Figure 8.4). At this point, growing a single crystal proved a serious challenge as the growing rate of this phase is extremely fast; a fast growing rate generates defects in the crystal that act as nucleation points. After several attempts of cycling pressure and temperature, a suitable single crystal for X-ray diffraction was obtained (Figure 8.4) and measured at ca. 0.5 GPa. Controlled and constant slow heating over the course of several hours in a small incubator proved to be crucial for the growth of this form. 


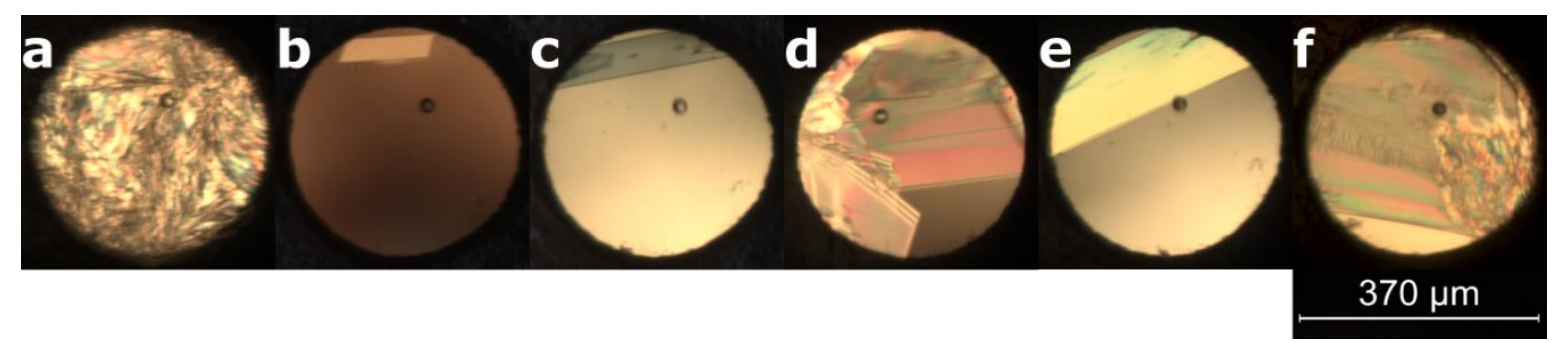

Figure 8.4. Optical microscopy images of the high-pressure growth of form II-c in a DAC. (a) DAC closed, polycrystalline material at ca. $0.05 \mathrm{GPa}$; (b) A single crystal obtained by cycling temperature; (c) The single crystal growing at $323 \mathrm{~K}$; (d) Turning off the heat source induces the growth of multiple crystals; (e) New single crystal growing under constant heat of $323 \mathrm{~K}$; (f) Final stage - predominantly single crystal at 0.05 GPa.

\subsubsection{Form II-d: crystallisation of [ $\left.\mathrm{C}_{10} \mathrm{mim}\right] \mathrm{Cl}$ pseudo-hemihydrate at $0.27 \mathrm{GPa}$}

In order to improve humidity control, a nitrogen glove box was used for sample preparation. The compound was dried for 5 hours at $333 \mathrm{~K}$ under vacuum, and then transferred into a DAC inside a nitrogen glove box operating at a relative humidity of $6.4 \%$. The DAC was sealed inside the glove box and pressure increased to $0.13 \mathrm{GPa}$. Crystallisation was induced by keeping the DAC at $0.35 \mathrm{GPa}$ and $277 \mathrm{~K}$ for 12 hours. The optimal single-crystal growth conditions proved difficult to find. The crystals were very sensitive to temperature and pressure changes and thus the multi-nucleation phenomenon was ubiquitous (Figure 8.5). After extensive testing the following procedure was established: starting from the crystalline powder at $0.35 \mathrm{GPa}$, the pressure was cycled several times to dissolve all material but a few crystals. The temperature was subsequently cycled using a heatgun at $323 \mathrm{~K}$ to dissolve all but one single crystal. The DAC was then placed into an incubator and the temperature was decreased from 306 to $298 \mathrm{~K}$ at a rate of 1 K/day. The final crystal (Figure 8.5.f) was measured at 0.27 GPa by means of single-crystal X-ray diffraction.

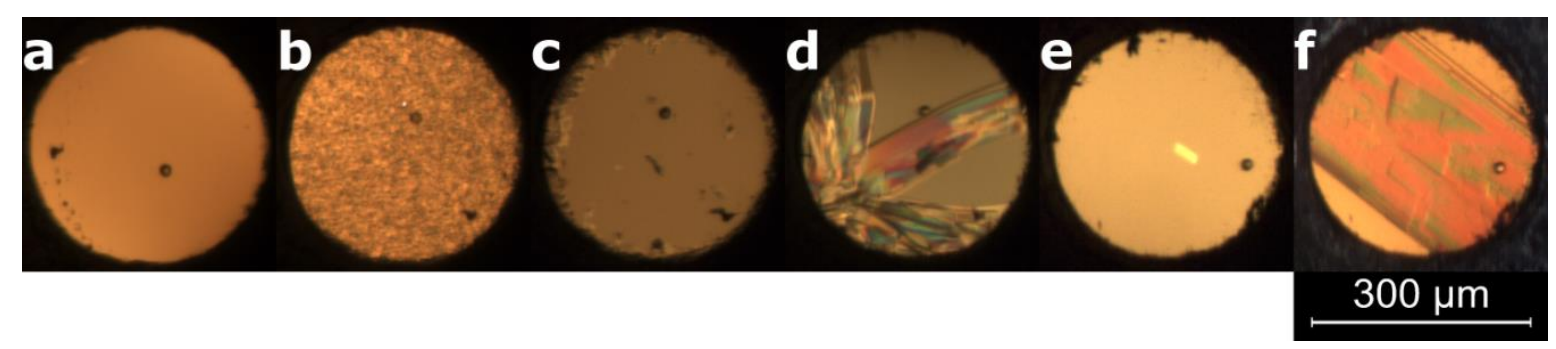

Figure 8.5. Optical microscopy images of the high-pressure growth of form II-d in a DAC. (a) DAC Closed. Pressure: $0.18 \mathrm{GPa}$; (b) Crystalline powder at $0.35 \mathrm{GPa}$ obtained after a few hours at $277 \mathrm{~K}$; (c) The crystalline powder dissolving by cycling pressure; (d) A slight increase of the pressure promotes fast growth of the remaining crystals; (e) A single crystal obtained by cycling pressure and temperature. The DAC was placed into an incubator for 10 days with a starting pressure of $0.09 \mathrm{GPa}$; (f) Final stage at $0.27 \mathrm{GPa}$.

\subsubsection{Form III: Crystallisation of [ $\left.\mathrm{C}_{10} \mathrm{mim}\right] \mathrm{Cl}$ trihydrate at $1.14 \mathrm{GPa}$}

A ca. $0.5 \mathrm{M}$ aqueous solution of $\left[\mathrm{C}_{10 \mathrm{mim}}\right] \mathrm{Cl}$, corresponding to a water content of $c a .88 \%$ $(\mathrm{w} / \mathrm{w})$, was prepared and loaded into a DAC. By increasing pressure the clear solution crystallises instantaneously at about $0.95 \mathrm{GPa}$ (Figure 8.6). The solid to liquid transition takes place at ca. 0.60 
GPa while releasing the pressure. A second crystallisation path consists of increasing the pressure above the transition point to a pressure of $c a .0 .7 \mathrm{GPa}$ and promoting crystallisation either quickly by putting the DAC in a cold environment (253-277 K), or slowly by leaving the DAC at ambient temperature; nucleation is achieved after a certain latency period. A single crystal was successfully grown by cycling temperature at $c a .0 .55 \mathrm{GPa}$. The pressure was subsequently increased slowly to favour the growth of the crystal. The crystal occupied almost half of the sample chamber in the final growth stage at ca. 1.14 GPa (Figure 8.6). The single crystal was measured by single-crystal Xray diffraction at the same pressure.

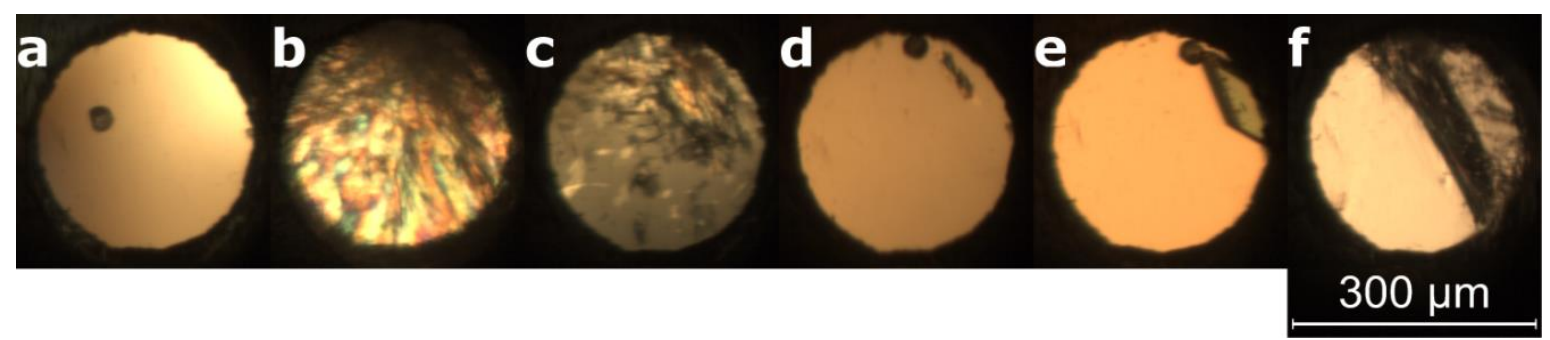

Figure 8.6. Optical microscopy images of the high-pressure growth of form III at 1.14 GPa. (a) Clear solution at ca. $0.7 \mathrm{GPa}$; (b) Polycrystalline material at $0.7 \mathrm{GPa}$ after $20 \mathrm{~min}$ at $263 \mathrm{~K}$; (c) Heating at $373 \mathrm{~K}$ and $0.7 \mathrm{GPa}$; (d) Cooling at $363 \mathrm{~K}$; (e) Single crystal growing on cooling at $353 \mathrm{~K}$; (f) Final crystal 1.14 GPa.

\subsubsection{Form III: Crystallisation of [ $\left.\mathrm{C}_{10 \mathrm{mim}}\right] \mathrm{Cl}$ trihydrate at $0.6 \mathrm{GPa}$}

In order to investigate the effects of concentration on the crystallisation outcome, a further high-pressure crystallisation experiment of a ca. $1 \mathrm{M}$ aqueous solution of $\left[\mathrm{C}_{10 \mathrm{mim}}\right] \mathrm{Cl}$, corresponding to a water content of $c a .80 \%(\mathrm{w} / \mathrm{w})$, was carried out. The pressure was increased to $0.58 \mathrm{GPa}$ and crystallisation was induced within 2 hours at $263 \mathrm{~K}$; we note that at the same pressure conditions no crystallisation occurred after 19 hours at $277 \mathrm{~K}$. A single crystal was grown by cycling temperature at slightly higher pressures (ca. $0.63 \mathrm{GPa}$, Figure 8.7). While the crystal was growing, the pressure dropped until the system reached the phase boundary conditions at $c a .0 .55$ GPa. The final pressure at which the crystal was measured was $0.60 \mathrm{GPa}$.

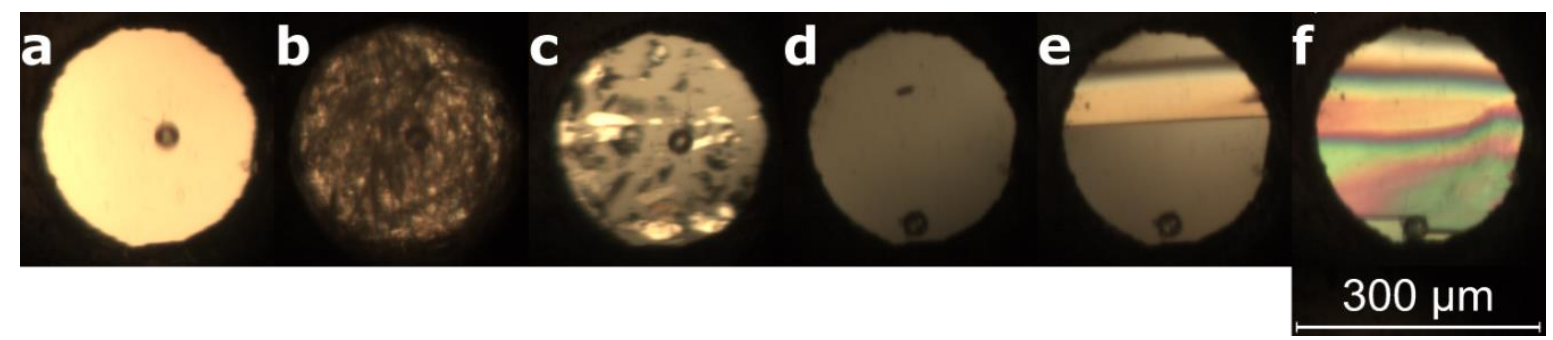

Figure 8.7. Optical microscopy images of the high-pressure growth of form III at $0.60 \mathrm{GPa}$. (a) Clear solution at ca. $0.12 \mathrm{GPa}$; (b) Polycrystalline material at $0.58 \mathrm{GPa}$ after 2 hours at $263 \mathrm{~K}$; (c) Crystals dissolving at 0.58 GPa and $323 \mathrm{~K}$ using a heat source; (d) Crystal at $0.58 \mathrm{GPa}$ under $323 \mathrm{~K}$; (e) Single crystal growing at $0.63 \mathrm{GPa}$;

(f) Final crystal at $0.6 \mathrm{GPa}$. 


\subsubsection{X-ray crystallography}

Diffraction data for form II-d were collected on an in-house modified mardtb desktop diffractometer equipped with a mar345 image plate detector and an Incoatec (I $\mu$ S) microfocus Xray source using $\operatorname{Ag~K} \alpha$ radiation of $\lambda=0.56085 \AA$. Diffraction experiments of all other forms were performed using a Bruker AXS SMART Apex II CCD diffractometer equipped with Mo K $\alpha$ sealedtube radiation of $\lambda=0.71073 \AA$ and an Oxford Cryosystems Cobra low-temperature device. The low-temperature crystalline phases are probably stable at ambient temperature under negligible air humidity $(<2 \%)$. After a few hours at ambient air humidity, measured at $30 \%$ in our laboratory, all single crystals underwent a phase transition to gel-like lyotropic phases. It was imperative to protect the crystals before and after crystal growth at low temperature and immersion oil was used for this purpose; single crystals were quickly transferred to the $\mathrm{X}$-ray machine and placed under the cold stream.

\subsubsection{Data processing}

Data integration and global-cell refinement were performed using the program SAINT.27 High-pressure data processing was performed according to the procedure by Dawson et al. ${ }^{28}$ The program $S A D A B S^{29}$ was used to correct for absorption and systematic errors. For the high-pressure data, the program $S H A D E^{30}$ was additionally used. All structures were solved with macromolecular phasing methods using SHELXD ${ }^{31}$. Partial fragment seeding using 1,3dimethylimidazolium was employed to solve the structure of form II-c. Full-matrix least-squares structure refinement against $F^{2}$ was performed using SHELXL2014 ${ }^{31}$ through the SHELXLE GUI. ${ }^{32}$ Stereochemical restraints for the cations were applied to increase the robustness of the refinement: restraints were generated by the GRADE program using the GRADE Web server ${ }^{33}$. A GRADE dictionary for SHELXL contains target values and standard deviations for 1,2-distances (DFIX) and 1,3-distances (DANG), as well as restraints for planar groups (FLAT). Anisotropic displacement parameters were refined using the new rigid bond restraint (RIGU) implemented in the SHELXL2014 program. ${ }^{34}$ All $\mathrm{H}$-atoms of [ $\left.\mathrm{C}_{10 \mathrm{mim}}\right]^{+}$were placed geometrically and allowed to ride on the parent atoms. $\mathrm{H}$-atoms belonging to ordered water molecules were clearly visible in difference Fourier maps and their positions were refined subject to distance restraints. For the remaining structures $\mathrm{H}$-atoms were placed based on considerations of $\mathrm{H}$-bonding patterns and refined subject to restraints. $U_{\text {iso }}(\mathrm{H})$ values were assigned in the range 1.2-1.5 times $U_{\text {eq }}$ of the parent atom. Structures of form II exhibited some type of disorder of one cation, one anion and one water molecule, $\mathrm{O}(3)$. Full details on the treatment of disorder can be found below. 


\subsubsection{Treatment of disorder}

The very good quality of low-temperature data of form I permits to locate a rotating $\mathrm{C}(6)$ methyl group with six peaks in the difference Fourier maps corresponding to $\mathrm{H}$ atoms. Two groups of $\mathrm{H}$ atoms were placed geometrically and allowed to ride on the parent atom. Both groups have equal site-occupancy factors of 0.5 .

All form II structures exhibit disorder affecting water molecules, the imidazolium ring of Res3 and the anion $\mathrm{Cl}(3)$ :

- For the low-temperature structures, the positions of the disordered non-hydrogen atoms were resolved in the density maps. Site-occupancy factors of all disordered atoms were refined, and then subsequently fixed only for water molecules.

- For the high-pressure structures, the imidazolium ring of Res3 was modelled to fit the electron density maps. Due to the lower quality of the data, site-occupancy factors of all disordered atoms were fixed to the initially refined values.

- For both low-temperature and high-pressure structures, $\mathrm{H}$ atoms belonging to disordered water were not placed during refinement but were taken into account for the calculation of $F_{000}$ and derived properties.

\subsection{Results and discussion}

A total of eight crystal structures were refined, three obtained using low-temperature crystallisation conditions and five at high pressure. Crystallographic data are summarised in Table 8.1. Form I corresponds to a monohydrate and was obtained under both low-temperature and high-pressure conditions. With ten analogous structures reported in the Cambridge Structural Database, ${ }^{9}$ form I is the most common monohydrate (the CSD, V. 5.35 including updates up to May 2014 was searched using no filters). Form II crystallises with a variable water content of 0.95 and 0.93 water molecules per cation at low temperature and 0.47-0.5 at high pressure. The CSD contains two entries for analogous structures of form II. Form III corresponds to a high-pressure trihydrate never reported previously for long-alkyl chain imidazolium-based ILs or other ILs. For our database search, analogous structures are not only defined as structures having similar unit cell parameters, but also as structures that are homeotypic at least at the level of a 2D network (see below for details). Table 8.3 summarises the observed forms of all [ $\left.\mathrm{C}_{n} \operatorname{mim}\right][X] \operatorname{ILs}(n \geq 8, X \equiv$ halide anion) together with their crystallisation conditions reported in the literature including the structures reported in this work.

Forms I-III crystallise in the triclinic centrosymmetric space group. Similar unit-cell settings were chosen to facilitate structural comparison of all forms. The structures obtained at low temperature are of good quality. Data completeness of the high-pressure structure is between 33 
and $48 \%$; the low completeness is on one hand due to the limited access to reciprocal space caused by the geometry of the DAC, exacerbated here by the low crystal symmetry, and on the other hand to the crystals' quality and size. Whereas the structure of form III-b was easily solved thanks to the good data collected at 1.14 GPa, data of form I-b and especially data of form II-c were far from ideal. Considerable time and effort were required to obtain crystals of good diffraction quality; despite the low data-to-parameter ratio for the high-pressure data, the refinement results are very satisfactory, with the exception of form II-d for which the maximum resolution is $1.3 \AA$ and the $R$ factor is the highest with a value of 0.135 ; this is directly related to the difficulties of growing crystals of this form and hence the limited crystal quality. Despite the high $R$-factor for this form, all structural features can be described with confidence. In the following sections, the X-ray structures are described and compared in more details.

For the description of all structures, a positive C2-N1-C7-C8 torsion angle was chosen (Figure 8.1).

Table 8.1. Crystallographic data for [C10mim] $\mathrm{Cl}$ structures discussed in this chapter. The same Roman numeral is given to structures with similar unit cell parameters. The letter following the Roman numeral indicates variation in water content and/or position in different crystal structures. All structures have been solved from single-crystal data.

\begin{tabular}{|c|c|c|c|c|c|c|c|c|}
\hline Structure & I & I & II-a & II-b & II-c & II-d & III & III \\
\hline Formula & $\begin{array}{c}{\left[\mathrm{C}_{10 \mathrm{mim}}\right] \mathrm{Cl}} \\
1\left(\mathrm{H}_{2} \mathrm{O}\right)\end{array}$ & $\begin{array}{c}{\left[\mathrm{C}_{10 \mathrm{mim}}\right] \mathrm{Cl}} \\
1\left(\mathrm{H}_{2} \mathrm{O}\right)\end{array}$ & $\begin{array}{c}{\left[\mathrm{C}_{10 \mathrm{mim}}\right] \mathrm{Cl},} \\
0.95\left(\mathrm{H}_{2} \mathrm{O}\right)\end{array}$ & $\begin{array}{c}\text { [C10mim]Cl, } \\
0.97\left(\mathrm{H}_{2} \mathrm{O}\right)\end{array}$ & $\begin{array}{c}\text { [C10mim]Cl, } \\
0.5\left(\mathrm{H}_{2} \mathrm{O}\right)\end{array}$ & $\begin{array}{c}\text { [C1omim]Cl, } \\
\left.0.47 \mathrm{H}_{2} \mathrm{O}\right)\end{array}$ & $\begin{array}{c}\text { [C10mim]Cl, } \\
3\left(\mathrm{H}_{2} \mathrm{O}\right)\end{array}$ & $\begin{array}{c}{\left[\mathrm{C}_{10 m i m}\right] \mathrm{Cl},} \\
3\left(\mathrm{H}_{2} \mathrm{O}\right)\end{array}$ \\
\hline Space group & $P-1$ & $P-1$ & $P-1$ & $P-1$ & $P-1$ & $P-1$ & $P-1$ & $P-1$ \\
\hline$a / \AA ̊$ & $5.3607(2)$ & $5.3387(19)$ & $7.2807(2)$ & $7.344(6)$ & 7.3494(5) & $7.3264(18)$ & $5.3513(5)$ & $5.2844(5)$ \\
\hline$b / \AA ̊ A$ & $7.7232(2)$ & 7.7592(12) & $8.4710(2)$ & $8.508(7)$ & $8.4551(5)$ & $8.371(2)$ & $7.2152(8)$ & $7.0547(4)$ \\
\hline$c / \AA ̊$ & $20.4674(6)$ & $20.428(5)$ & 43.0059(11) & 43.39(4) & $42.735(7)$ & $42.879(16)$ & $22.706(5)$ & $22.364(2)$ \\
\hline$\alpha /^{\circ}$ & $82.1449(12)$ & $82.056(17)$ & $85.0460(13)$ & $84.77(5)$ & $83.125(9)$ & $82.900(7)$ & 83.711(14) & $83.949(6)$ \\
\hline$\beta /^{\circ}$ & $87.2216(12)$ & $87.16(2)$ & $89.8188(14)$ & $89.32(5)$ & $89.000(9)$ & $89.106(11)$ & $89.209(13)$ & $88.487(7)$ \\
\hline$\gamma / /^{\circ}$ & 78.9156(12) & 79.09(2) & $70.9453(13)$ & 70.73(5) & $69.871(4)$ & $69.909(8)$ & $82.429(7)$ & $83.478(6)$ \\
\hline$V / \AA^{3}$ & $823.56(4)$ & $822.7(4)$ & $2496.80(11)$ & $2548(4)$ & $2474.6(5)$ & $2449.7(13)$ & $863.8(2)$ & $823.64(12)$ \\
\hline$Z^{\prime}$ & 1 & 1 & 3 & 3 & 3 & 3 & 1 & 1 \\
\hline$D_{\text {cald }} / \mathrm{g} \mathrm{cm}^{-3}$ & 1.116 & 1.118 & 1.100 & 1.077 & 1.078 & 1.087 & 1.203 & 1.262 \\
\hline Pressure & $0.1 \mathrm{MPa}$ & $0.2 \mathrm{GPa}$ & $0.1 \mathrm{MPa}$ & $0.1 \mathrm{MPa}$ & $0.05 \mathrm{GPa}$ & $0.27 \mathrm{GPa}$ & $0.6 \mathrm{GPa}$ & $1.14 \mathrm{GPa}$ \\
\hline$T / K$ & $263(2)$ & $293(2)$ & 200(2)K & 263(2)K & $293(2)$ & $293(2)$ & $293(2)$ & $293(2)$ \\
\hline $\begin{array}{l}\text { Meas//uniq/obs. } \\
\text { Reflections }\end{array}$ & $\begin{array}{c}7370,3047 \\
2426\end{array}$ & $\begin{array}{c}3044,682 \\
528\end{array}$ & $\begin{array}{c}23611,6760 \\
5206\end{array}$ & $\begin{array}{c}8895,3028 \\
1797\end{array}$ & $\begin{array}{c}12099,1592 \\
1159\end{array}$ & $\begin{array}{l}4352,1105 \\
921\end{array}$ & $\begin{array}{c}3646,945 \\
687\end{array}$ & $\begin{array}{c}6348,1145 \\
866\end{array}$ \\
\hline$d_{\max } / \AA$ & 0.800 & 1.000 & 0.930 & 1.196 & 1.150 & 1.293 & 0.860 & 0.83 \\
\hline$(\operatorname{Sin}(\theta) / \lambda) / \AA^{-1}$ & 0.625 & 0.500 & 0.538 & 0.418 & 0.435 & 0.387 & 0.582 & 0.602 \\
\hline $\begin{array}{l}\text { Completeness } \\
\text { to } d_{\max }\end{array}$ & 0.904 & 0.393 & 0.976 & 0.984 & 0.475 & 0.467 & 0.332 & 0.382 \\
\hline$R_{\text {int }}$ & 0.0173 & 0.0504 & 0.0468 & 0.0724 & 0.0589 & 0.0881 & 0.0310 & 0.0364 \\
\hline$R_{1} c^{c}$ & 0.0372 & 0.0692 & 0.0531 & 0.0499 & 0.0682 & 0.1480 & 0.0463 & 0.0494 \\
\hline$w R_{2}^{d}$ & 0.1003 & 0.2173 & 0.1450 & 0.1231 & 0.2060 & 0.3127 & 0.1065 & 0.1433 \\
\hline
\end{tabular}


Table 8.2. Summary of the crystallisation conditions of the [Cnmim]X structures $(n \geq 8, X \equiv$ halide anion) reported in the literature and in this work.

\begin{tabular}{|c|c|c|c|c|c|c|c|c|}
\hline \multirow{2}{*}{ Author } & \multirow{2}{*}{$\begin{array}{c}\text { CSD } \\
\text { REFCODE }\end{array}$} & \multirow{2}{*}{ Formula } & \multicolumn{3}{|c|}{ Experiments } & \multicolumn{3}{|c|}{ Results - Analogues } \\
\hline & & & Setup & Solvent & Conditions & Form I & Form II & Form III \\
\hline Chen et al. ${ }^{1}$ & YORNOJ01 & $\begin{array}{c}{\left[\mathrm{C}_{14 \mathrm{mim}}\right] \mathrm{Br}} \\
1 \mathrm{H}_{2} \mathrm{O}\end{array}$ & Slow evaporation & Chloroform & $\mathrm{RT}^{\mathrm{a}}$ for $c a .5$ days & X & & \\
\hline \multirow{3}{*}{$\begin{array}{c}\text { Downard } e t \\
a l .{ }^{2}\end{array}$} & BELKUZ & $\begin{array}{c}{\left[\mathrm{C}_{18 \mathrm{mim}}\right] \mathrm{Cl} \cdot} \\
1 \mathrm{H}_{2} \mathrm{O}\end{array}$ & Slow evaporation & 1,2-Dichloroethane & RT & & $x$ & \\
\hline & BELLIO01 & $\begin{array}{c}{\left[\mathrm{C}_{144 \mathrm{mim}}\right] \mathrm{Cl} \cdot} \\
1 \mathrm{H}_{2} \mathrm{O}-\mathrm{I}\end{array}$ & Recrystallisation & Ethyl acetate & Hot solvent & & $x$ & \\
\hline & BELLIO & $\begin{array}{c}{\left[\mathrm{C}_{14 \mathrm{mim}}\right] \mathrm{Cl} \cdot} \\
1 \mathrm{H}_{2} \mathrm{O}-\mathrm{II}\end{array}$ & Heating & 1,2-Dichloroethane & $50^{\circ} \mathrm{C} / 48 \mathrm{~h}$ & $X$ & & \\
\hline $\begin{array}{l}\text { Getsis and } \\
\text { Mudring }\end{array}$ & QATNIJ & $\begin{array}{c}{\left[\mathrm{C}_{12} \mathrm{mim}\right] \mathrm{Br}} \\
1 \mathrm{H}_{2} \mathrm{O}\end{array}$ & Recrystallisation & Ethyl acetate & / & $x$ & & \\
\hline \multirow{2}{*}{$\begin{array}{l}\text { Getsis and } \\
\text { Mudring }\end{array}$} & QATNIJ01 & $\begin{array}{c}{\left[\mathrm{C}_{12} \mathrm{mim}\right] \mathrm{Br}} \\
1 \mathrm{H}_{2} \mathrm{O}\end{array}$ & $\begin{array}{l}\text { Isothermal } \\
\text { evaporation }\end{array}$ & Acetic anhydride & RT & $X$ & & \\
\hline & YORNOJ & $\begin{array}{c}{\left[\mathrm{C}_{14} \mathrm{mim}\right] \mathrm{Br}} \\
1 \mathrm{H}_{2} \mathrm{O}\end{array}$ & $\begin{array}{l}\text { Isothermal } \\
\text { evaporation }\end{array}$ & Acetic anhydride & RT & $\mathrm{X}$ & & \\
\hline Guillet et al..$^{5}$ & HAKWOG & $\begin{array}{c}{\left[\mathrm{C}_{12} \mathrm{mim}\right] \mathrm{Cl} \cdot} \\
1 \mathrm{H}_{2} \mathrm{O}\end{array}$ & Slow evaporation & $\begin{array}{c}1,2- \\
\text { Dichloroethane/in } \\
\text { contact with } \\
\text { atmospheric } \\
\text { moisture }\end{array}$ & RT & X & & \\
\hline Wei et al. ${ }^{6}$ & SOXGOC & $\begin{array}{c}{\left[\mathrm{C}_{16} \mathrm{mim}\right] \mathrm{Br} \cdot} \\
1 \mathrm{H}_{2} \mathrm{O}\end{array}$ & Slow evaporation & Ethyl acetate & 2 weeks & $x$ & & \\
\hline Zhao et al. ${ }^{7}$ & SOXGOC01 & $\begin{array}{c}{\left[\mathrm{C}_{16} \mathrm{mim}\right] \mathrm{Br}} \\
1 \mathrm{H}_{2} \mathrm{O}\end{array}$ & Not available & & & $\mathrm{X}$ & & \\
\hline Zhao et al. ${ }^{8}$ & SOXGOC02 & $\begin{array}{c}{\left[\mathrm{C}_{16} \mathrm{mim}\right] \mathrm{Br}} \\
1 \mathrm{H}_{2} \mathrm{O}\end{array}$ & Slow cooling & Not available & $\begin{array}{l}\text { Sample dried } 24 \mathrm{~h} \\
\text { under vacuum at } \\
323 \mathrm{~K}\end{array}$ & $x$ & & \\
\hline \multirow{8}{*}{ This work } & & $\begin{array}{c}{\left[\mathrm{C}_{10 \mathrm{mim}}\right] \mathrm{Cl} \cdot} \\
1 \mathrm{H}_{2} \mathrm{O}\end{array}$ & Linkam stage & $6-8 \%(w / w) \mathrm{H}_{2} \mathrm{O}$ & Slow cooling & $X$ & & \\
\hline & & $\begin{array}{c}{\left[\mathrm{C}_{10 \mathrm{mim}}\right] \mathrm{Cl} \cdot} \\
1 \mathrm{H}_{2} \mathrm{O}\end{array}$ & $\mathrm{DAC}^{\mathrm{b}}$ & $6-8 \%(w / w) \mathrm{H}_{2} \mathrm{O}$ & $\begin{array}{l}\text { Formed above } 0.3 \\
\text { GPa }\end{array}$ & $x$ & & \\
\hline & & $\begin{array}{c}{\left[\mathrm{C}_{10 \mathrm{mim}}\right] \mathrm{Cl} \cdot} \\
0.97 \mathrm{H}_{2} \mathrm{O}\end{array}$ & Linkam stage & $<6 \%(w / w) \mathrm{H}_{2} \mathrm{O}$ & $\begin{array}{l}\text { Preheated / fast } \\
\text { cooling }\end{array}$ & & $x$ & \\
\hline & & $\begin{array}{c}{\left[\mathrm{C}_{10 \mathrm{mim}}\right] \mathrm{Cl} \cdot} \\
0.93 \mathrm{H}_{2} \mathrm{O}\end{array}$ & Linkam stage & $<6 \%(w / w) \mathrm{H}_{2} \mathrm{O}$ & $\begin{array}{l}\text { Preheated / fast } \\
\text { cooling }\end{array}$ & & $x$ & \\
\hline & & $\begin{array}{c}{\left[\mathrm{C}_{10 \mathrm{mim}}\right] \mathrm{Cl} \text {. }} \\
0.5 \mathrm{H}_{2} \mathrm{O}\end{array}$ & DAC & $\ll 6 \%(w / w) \mathrm{H}_{2} \mathrm{O}$ & $\begin{array}{l}\text { Sample dried } 3 \mathrm{~h} \\
\text { under vacuum at } \\
333 \mathrm{~K}\end{array}$ & & $x$ & \\
\hline & & $\begin{array}{c}{\left[\mathrm{C}_{10 \mathrm{mim}}\right] \mathrm{Cl} \cdot} \\
0.48 \mathrm{H}_{2} \mathrm{O}\end{array}$ & DAC + glove box & $<<6 \%(w / w) \mathrm{H}_{2} \mathrm{O}$ & $\begin{array}{l}\text { Sample dried } 5 \mathrm{~h} \\
\text { under vacuum at } \\
333 \mathrm{~K}\end{array}$ & & $x$ & \\
\hline & & $\begin{array}{c}{\left[\mathrm{C}_{10 \mathrm{mim}}\right] \mathrm{Cl} \cdot} \\
3 \mathrm{H}_{2} \mathrm{O}\end{array}$ & DAC & $1 \mathrm{M}$ aq. solution & $\begin{array}{c}\text { Stable above } 0.55 \\
\text { GPa }\end{array}$ & & & $x$ \\
\hline & & $\begin{array}{c}{\left[\mathrm{C}_{10 \mathrm{mim}}\right] \mathrm{Cl} \cdot} \\
3 \mathrm{H}_{2} \mathrm{O}\end{array}$ & DAC & $0.5 \mathrm{M}$ aq. solution & $\begin{array}{c}\text { Stable above } 0.55 \\
\text { GPa }\end{array}$ & & & $x$ \\
\hline
\end{tabular}

${ }^{\mathrm{a}} \mathrm{RT}=$ room temperature. ${ }^{\mathrm{b}} \mathrm{DAC}=$ diamond-anvil cell. 


\subsubsection{Crystal structure of form I}

Form I is the most often encountered structure of a hydrate of a long alkyl chain imidazolium-based halide IL in the CSD. An important point to retain from Table 8.2 it that all reported form I structures were obtained using slow crystallisation processes, in line with our experimental findings.

The structure of form I has been described several times previously. ${ }^{1-8}$ The following are the main structural features characterising all reported structures, including our determination. The structure of form I consists of bilayers, in which the cations are alternating from layer to layer creating hydrophobic and hydrophilic regions (Figure 8.2). The hydrophobic region is formed by interactions between interdigitated side chains having a linear all-anti conformation. The hydrophilic region is formed by an extensive H-bonded network between the imidazolium ring, the chloride anion and the water molecule. The main H-bonded contacts include two short interactions between the chloride anion and the water molecule $[\mathrm{O} \cdots \mathrm{Cl}=3.3231(17)$ and 3.2457(17) $\AA$ at low temperature, 3.272(19) and 3.286(19) at high pressure] thereby forming an infinite zigzag chain along the $\left[\begin{array}{lll}1 & 0 & 0\end{array}\right]$ base vector (see Figure 8.2.3). The imidazolium ring is connected to two chloride anions through $\mathrm{C}(4)-\mathrm{H} \cdots \mathrm{Cl}$ and $\mathrm{C}(5)-\mathrm{H}^{\cdots} \cdot \mathrm{Cl}^{\prime}$ contacts, forming an approximately planar H-bonded ring motif (Figure 8.8). The water molecule is accepting a H-bond from $\mathrm{C}(2)-\mathrm{H}$ of the imidazolium ring (Figure 8.8). Hence, the water molecule is a single H-bonded acceptor and fulfils its capacity as double H-bonded donor.

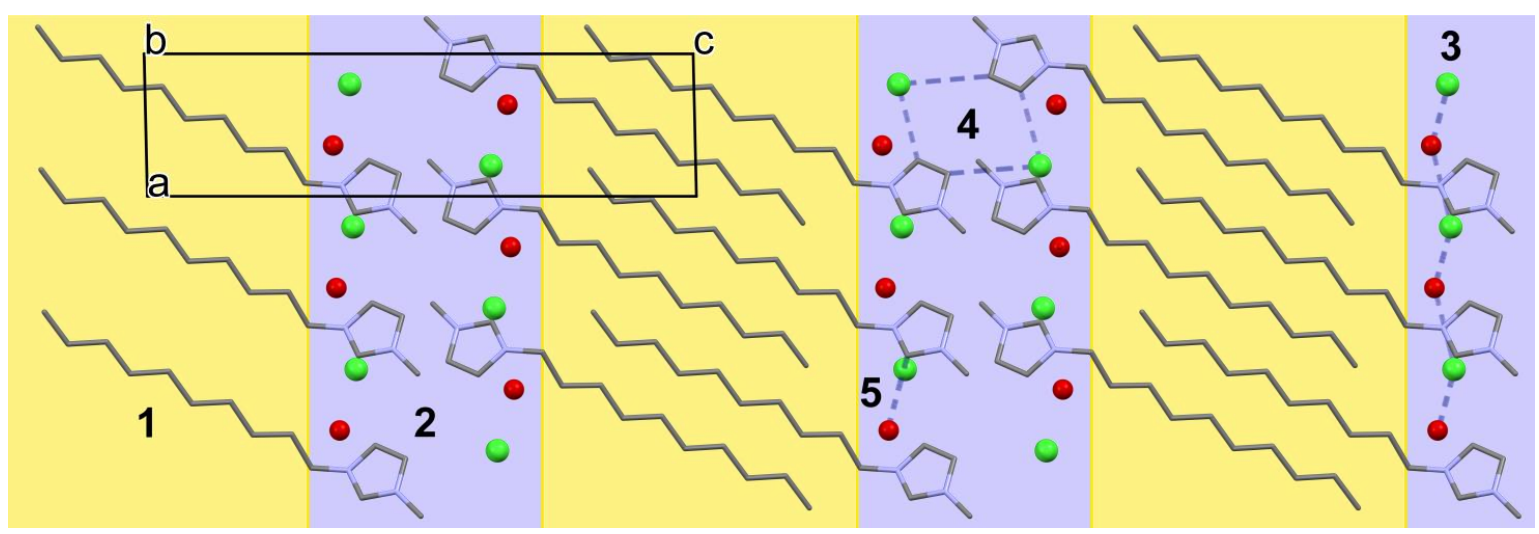

Figure 8.8. Crystal packing of [ $\left.\mathrm{C}_{10 \mathrm{mim}}\right] \mathrm{Cl}$ form I seen along the $\boldsymbol{b}$-axis. H-atoms have been omitted for clarity. (1) Hydrophobic layer with interdigitated cation side chains; (2) Hydrophilic layer; (3) O-H $\cdots \mathrm{Cl} \mathrm{H-}$ bonded chain along the $a$-axis; (4) H-bonded ring motif formed between $\mathrm{C}(4)-\mathrm{H}, \mathrm{C}(5)-\mathrm{H}$ and $[\mathrm{Cl}]_{2^{2-}}$; (5) Water imidazolium contact via $\mathrm{C}(2)-\mathrm{H}$.

Getsis and Mudring stated that in the solid state $\left[\mathrm{C}_{12} \mathrm{mim}\right] \mathrm{Br} \cdot \mathrm{H}_{2} \mathrm{O}^{4}$ is isotypic with $\left[\mathrm{C}_{12} \mathrm{mim}\right] \mathrm{Cl} \cdot \mathrm{H}_{2} \mathrm{O}^{5}$ and that $\left[\mathrm{C}_{12} \mathrm{mim}\right] \mathrm{Br} \cdot \mathrm{H}_{2} \mathrm{O}$ is homeotypic with $\left[\mathrm{C}_{14} \mathrm{mim}\right] \mathrm{Br} \cdot \mathrm{H}_{2} \mathrm{O}_{4}{ }^{4}$ Starting from these observations, a detailed structural comparison was carried out using the XPac method ${ }^{35}$ and Hirshfeld surface analysis, ${ }^{36}$ with the aim of confirming and applying Getsis and Mudring's 
observation to all known structures of $\left[\mathrm{C}_{\mathrm{n} m i m}\right] \mathrm{X} \cdot \mathrm{H}_{2} \mathrm{O}(\mathrm{n} \geq 10, \mathrm{X} \equiv$ halide anion) crystallising as form I monohydrate, including the ones reported in this work. The XPac method is a powerful tool for assessing the degree of packing similarities between two or more crystal structures in a straightforward, efficient and rapid manner. Structural comparison is simplified to a similarity relationship of none-, zero-, one-, two- or three- dimensional supramolecular constructs assessed with a computed dissimilarity index. ${ }^{37}$ Seven crystal structures were compared using the $\left[\mathrm{C}_{10 \mathrm{mim}}\right]^{+}$ fragment common to all structures; the XPac program default values were used. The results of this analysis are summarised in Table 8.3. The 21 structural comparisons include three 3D and 18 2D supramolecular constructs. Other supramolecular constructs of lower dimensionality are not observed, indicating that all structures are highly similar. The three 3D supramolecular constructs describe comparisons of isotypic $\left[\mathrm{C}_{n} \mathrm{mim}\right][\mathrm{X}]$ structural pairs with identical alkyl chain length and have a dissimilarity index that ranges between 2.2 and 4.1: both structures of the $\left[\mathrm{C}_{10 \mathrm{mim}}\right] \mathrm{Cl} \cdot \mathrm{H}_{2} \mathrm{O}$ monohydrate obtained at low temperature and at high pressure, $\left[\mathrm{C}_{12} \mathrm{mim}\right][\mathrm{Cl}] \cdot \mathrm{H}_{2} \mathrm{O}$ with $\left[\mathrm{C}_{12} \mathrm{mim}\right][\mathrm{Br}] \cdot \mathrm{H}_{2} \mathrm{O}$, and $\left[\mathrm{C}_{14} \mathrm{mim}\right][\mathrm{Cl}] \cdot \mathrm{H}_{2} \mathrm{O}$ with $\left[\mathrm{C}_{14} \mathrm{mim}\right][\mathrm{Br}] \cdot \mathrm{H}_{2} \mathrm{O}$. The 18 2D supramolecular constructs describe homeotypic structures, where the missing similar third dimension is ascribed to the different alkyl chain lengths. The dissimilarity index values are between 0.8 and 4.5 .

Table 8.3. Summary of the dissimilarity index and supramolecular construct types obtained using XPac.

\begin{tabular}{|c|c|c|c|c|c|c|c|}
\hline \multirow{2}{*}{$\begin{array}{c}{\left[\mathrm{C}_{10} \mathrm{mim}\right] \mathrm{Cl} \cdot \mathrm{H}_{2} \mathrm{O}(\mathrm{LT})} \\
{\left[\mathrm{C}_{10} \mathrm{mim}\right] \mathrm{Cl} \cdot \mathrm{H}_{2} \mathrm{O}} \\
(\mathrm{HP}) \\
\end{array}$} & \multirow[b]{2}{*}{$2.2^{\mathrm{a}}$} & & \multirow{2}{*}{\multicolumn{2}{|c|}{ Dissimilarity index }} & \multirow{2}{*}{$\begin{array}{l}\text { low } \\
\text { low }\end{array}$} & \multirow{2}{*}{$\begin{array}{l}\text { 3D Supramolecular construct } \\
\text { 2D Supramolecular construct }\end{array}$} & \multirow{2}{*}{$\begin{array}{l}\text { high } \\
\text { high }\end{array}$} \\
\hline & & & & & & & \\
\hline$\left[\mathrm{C}_{12} \mathrm{mim}\right] \mathrm{Br} \cdot \mathrm{H}_{2} \mathrm{O}$ & $2.8^{\mathrm{b}}$ & $3.8^{\mathrm{b}}$ & & & & & \\
\hline$\left[\mathrm{C}_{12} \mathrm{mim}\right] \mathrm{Cl} \cdot \mathrm{H}_{2} \mathrm{O}$ & $3.1^{\mathrm{b}}$ & $4.0^{\mathrm{b}}$ & $4.1^{\mathrm{a}}$ & & & & \\
\hline$\left[\mathrm{C}_{144} \mathrm{mim}\right] \mathrm{Br} \cdot \mathrm{H}_{2} \mathrm{O}$ & $2.8^{\mathrm{b}}$ & $3.8^{\mathrm{b}}$ & $0.8^{\mathrm{b}}$ & $4.5^{b}$ & & & \\
\hline$\left[\mathrm{C}_{14} \mathrm{mim}\right] \mathrm{Cl} \cdot \mathrm{H}_{2} \mathrm{O}$ & $1.6^{\mathrm{b}}$ & $2.7^{\mathrm{b}}$ & $2.9^{\mathrm{b}}$ & $2.2^{\mathrm{b}}$ & & $3.0^{\mathrm{a}}$ & \\
\hline \multirow[t]{2}{*}[\mathrm{C}_{16}\mathrm{mim}]{$\mathrm{Br} \cdot \mathrm{H}_{2} \mathrm{O}$} & $2.5^{\mathrm{b}}$ & $3.5^{\mathrm{b}}$ & $1.1^{\mathrm{b}}$ & $4.1^{\mathrm{b}}$ & & $0.8^{\mathrm{b}}$ & $2.9^{\mathrm{b}}$ \\
\hline & $\begin{array}{c}{\left[\mathrm{C}_{10 \mathrm{mim}}\right] \mathrm{Cl} \cdot} \\
\mathrm{H}_{2} \mathrm{O}(\mathrm{LT})\end{array}$ & $\begin{array}{c}{\left[\mathrm{C}_{10} \operatorname{mim}\right] \mathrm{Cl} \cdot} \\
\mathrm{H}_{2} \mathrm{O}(\mathrm{HP})\end{array}$ & {$\left[\mathrm{C}_{12} \mathrm{mim}\right] \mathrm{Br} \cdot \mathrm{H}_{2} \mathrm{O}$} & {$\left[\mathrm{C}_{12} \mathrm{mim}\right] \mathrm{C}$} & $\mathrm{I}_{2} \mathrm{O}$ & {$\left[\mathrm{C}_{14} \mathrm{mim}\right] \mathrm{Br} \cdot \mathrm{H}_{2} \mathrm{O}$} & $\begin{array}{c}{\left[\mathrm{C}_{14} \mathrm{mim}\right] \mathrm{Cl} \cdot \mathrm{H}_{2}} \\
\mathrm{O}\end{array}$ \\
\hline
\end{tabular}

A further structural comparison based on Hirshfeld surface analysis implemented in the Crystal Explorer ${ }^{38}$ program has been carried out. Hirshfeld surface analysis is a powerful tool for elucidating and comparing intermolecular interactions and the packing environment in crystal structures. ${ }^{39}$ The structural comparison presented here is based on the visually informative twodimensional fingerprint plots. 


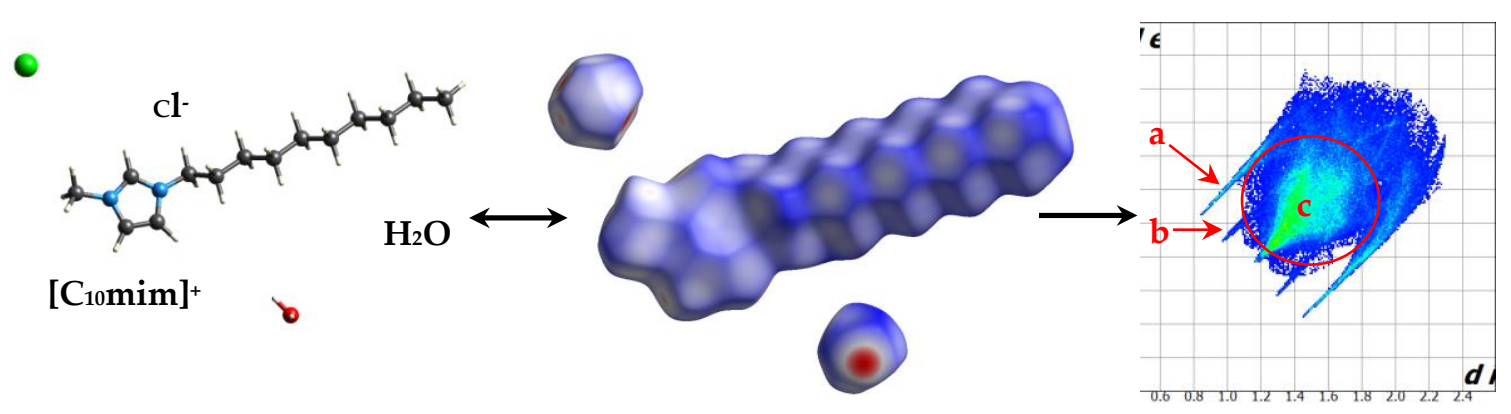

Figure 8.9. [ $\left.\mathrm{C}_{10 \mathrm{mim}}\right] \mathrm{Cl}$ cluster and its corresponding Hirshfeld surfaces mapped with the $\boldsymbol{d}_{\text {norm }}$ property. ${ }^{39}$

(a) $\mathrm{H} \cdots \mathrm{X}$ contacts; (b) $\mathrm{H} \cdots \mathrm{O}$ contacts; (c) $\mathrm{C}-\mathrm{H} \cdots \mathrm{H}-\mathrm{C}$ contacts

For the generation of the Hirshfeld surface a cluster, as opposed to the traditional individual molecule, was chosen. This enables the visualisation of all intermolecular contacts formed by the molecules in the cluster with neighbouring clusters in a single fingerprint plot for each structure. Each cluster contains a cation, a water molecule and an anion (Figure 8.9). For Hirshfeld surfaces generation a cluster was chosen so that the constituent molecules/ions are not directly connected to the others. This enables the generation of three Hirshfeld surfaces per cluster but only one fingerprint plot. The Hirshfeld surfaces generated in this way are identical to the ones that would be obtained for each individual molecule/ion. The main difference of this cluster approach with respect to the single-molecule one is that our fingerprint plots are necessarily much more symmetrical than those of the individual constituents. Inspection of the fingerprint plots (Figure 8.10) reveals a high degree of structural similarity, corroborating the results of XPac analysis presented earlier.

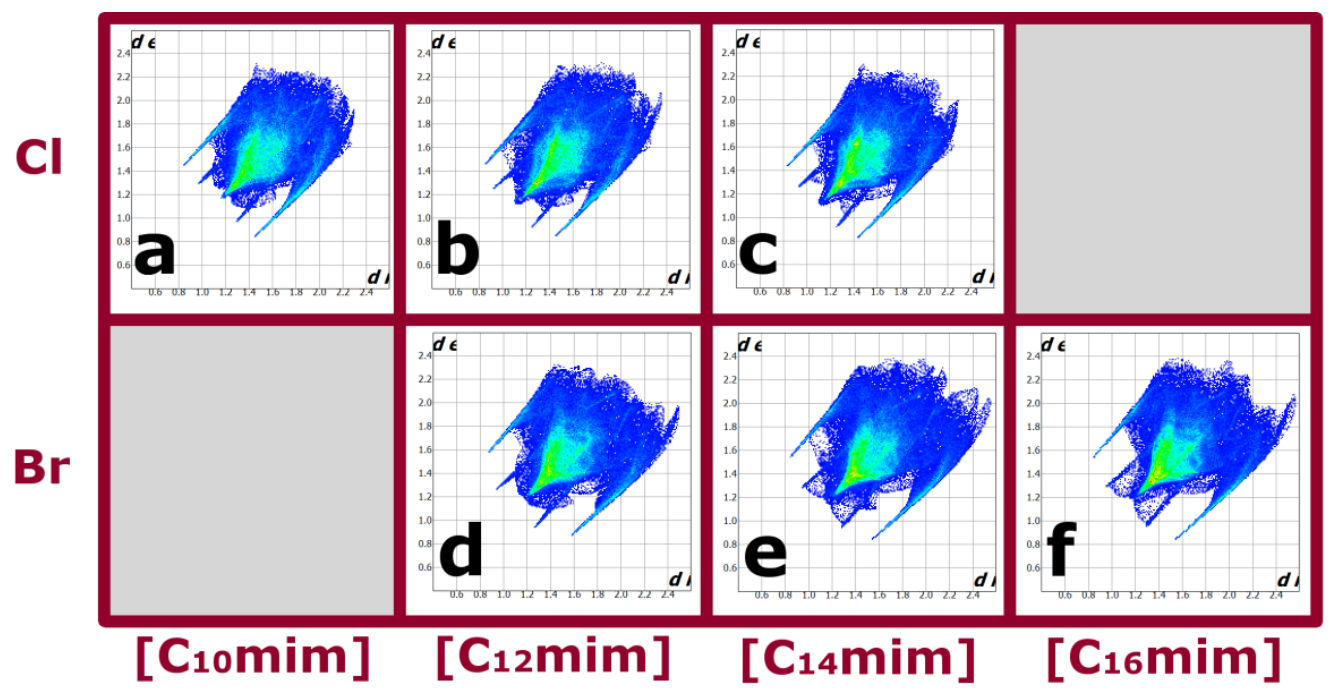

Figure 8.10. 2D fingerprint plots of the six structures of form I: (a) low-temperature structure of this work; (b) reference ${ }^{5}$; (c) reference ${ }^{2}$; (d) reference ${ }^{3} ;\left(\right.$ e) reference ${ }^{4} ;(\mathrm{f})$ reference $^{6}$. 


\subsubsection{Crystal structures of forms [II-a - II-d]}

The crystal structures of form II hydrates are homeotypic with respect to $\left[\mathrm{C}_{14} \mathrm{mim}\right] \mathrm{Cl} \cdot \mathrm{H}_{2} \mathrm{O}-\mathrm{I}$ and $\left[\mathrm{C}_{18 \mathrm{mim}}\right] \mathrm{Cl} \cdot \mathrm{H}_{2} \mathrm{O}$ reported by Downard et al.. ${ }^{2}$ However, unlike the latter two structures, all form II structures presented herein are not genuine monohydrates, hence in our study forms I and II are strictly speaking not polymorphic. Similarly to $\left[\mathrm{C}_{14} \mathrm{mim}\right] \mathrm{Cl} \cdot \mathrm{H}_{2} \mathrm{O}-\mathrm{I}$ and $\left[\mathrm{C}_{18 m i m}\right] \mathrm{Cl} \cdot \mathrm{H}_{2} \mathrm{O}$, form II crystallises with three symmetry independent cations and anions in the asymmetric unit. Whereas two of the cations (here named Res1 and Res2, where Res stands for residue, Figure 8.11) are linear and ordered, the third one (Res3) is bent and disordered over two positions. The chloride anion and water molecule associated with Res3 are disordered as well, giving rise to the structures' variable water content. This disorder causes the appearance of a second hydrophilic region compared to form I, thereby creating a double bilayered structure that extends along the $c$-axis. ${ }^{2}$ The double bilayer character gives rise to the characteristic long $c$-axis of all form II structures (Figure 8.12). At high pressure, further structural variability is introduced, with different occupancies for $\mathrm{O}(2)$, which is located in the (predominantly) ordered hydrophilic region, also observed (see Table 8.4 for details).

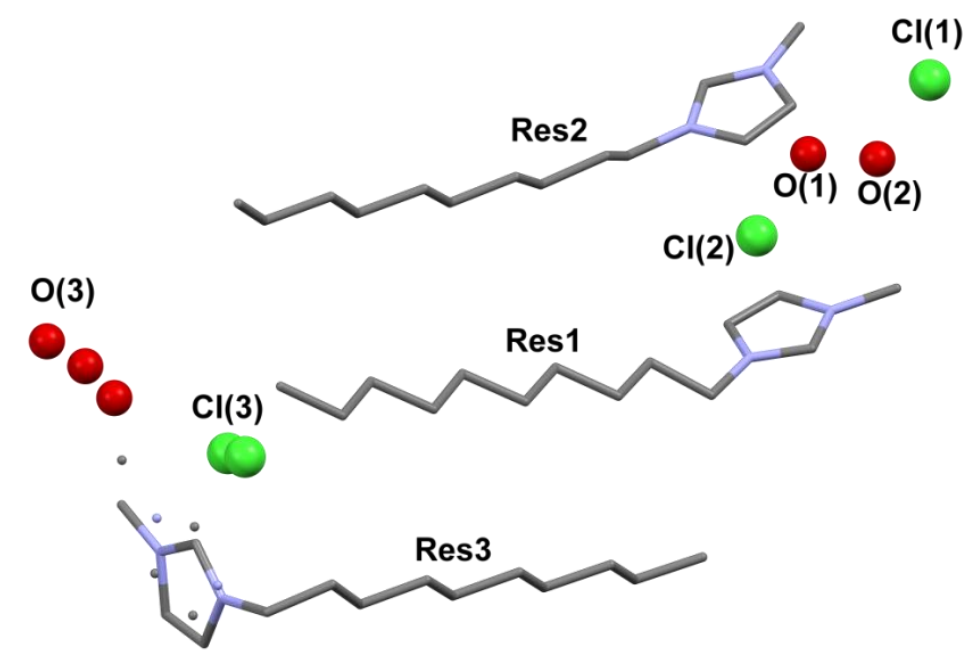

Figure 8.11. Asymmetric unit of [C $10 \mathrm{mim}] \mathrm{Cl}$ form II-a

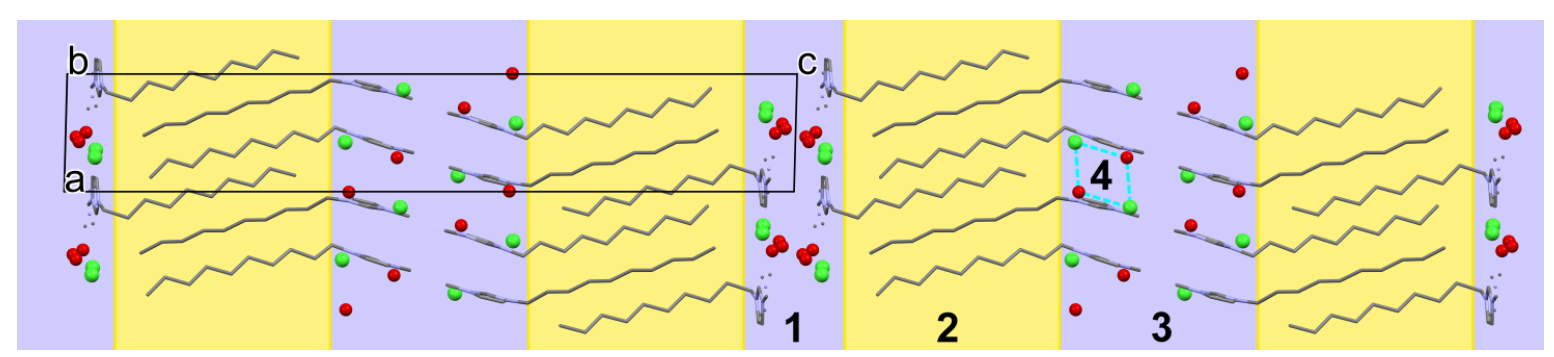

Figure 8.12. Crystal packing of [ $\left.\mathrm{C}_{10} \mathrm{mim}\right] \mathrm{Cl}$ form II-a seen along the $\boldsymbol{b}$-axis. H-atoms have been omitted for clarity. (1) Hydrophilic disordered layer; (2) Hydrophobic layer showing the interdigitated side chain; (3) Hydrophilic layer, ordered at low temperature. 
Although the crystals were grown under different experimental conditions and hence generalizations are difficult to make, it is interesting to note that the effect of reducing the water content from 0.95 to 0,47 molecules per cation in the structure manifests itself with the gradual removal of water molecules in the disordered region accompanied by partial occupancy of $\mathrm{O}(2)$. The $\mathrm{O}(1)$ water site is always fully occupied; this gives stability to the hydrophilic layer, which appears to be essential for structure stability of this form.

The H-bonded motifs involving Res1 and Res2 are similar to those described previously. ${ }^{2}$ Both $\mathrm{Cl}(1)$ and $\mathrm{Cl}(2)$ chloride anions form an isolated $\left\{\left[\mathrm{H}_{2} \mathrm{O}\right] \mathrm{Cl}_{2^{2}}{ }^{2-}\right.$ dimer with $\mathrm{O}(1)$ and $\mathrm{O}(2)$ water

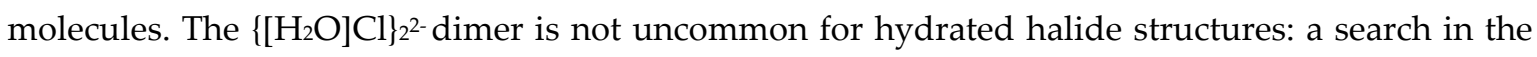
CSD (the CSD, V. 5.35 including updates to May 2014 was searched using no filters) shows that the dimer is occurring in 551 organic and inorganic crystal structures. This dimer is also present in the disordered region though the presence of disorder makes H-bond interpretations difficult. At low

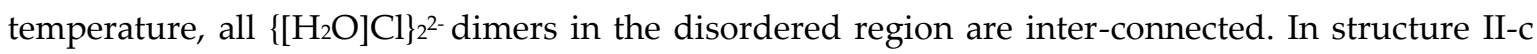
crystallised at high-pressure there are no water molecules in the disordered region. In structure II$d$ the $\left\{\left[\mathrm{H}_{2} \mathrm{O}\right] \mathrm{Cl}\right\}_{2^{2-}}$ dimer is present but in contrast to all other structures it is isolated.

Table 8.4. Summary of the differences in the site occupancies of all form II structures presented in this work.

\begin{tabular}{ccccc}
\hline & Form II-a & Form II-b & Form II-c & Form II-d \\
\hline Res 3 (imidazolium ring) & $1.000:$ & $1.000:$ & $1.000:$ & 0.500 \\
Part 1 (Res 3) & $0.682(12)$ & $0.716(16)$ & 0.500 & $1.000:$ \\
Part 2 (Res 3') & $0.318(12)$ & $0.284(16)$ & 0.500 & 0.500 \\
Cl(3) & 1.000 & 1.000 & 1.000 \\
Part 1 & $0.60(2)$ & $0.55(5)$ & 0.750 \\
Part 2 & $0.40(2)$ & $0.45(5)$ & 0.500 & 0.250 \\
O(1) & 1.000 & 1.000 & 0 \\
O(2) & 1.000 & 1.000 & 0.200 \\
O(3) & $0.850:$ & $0.897:$ & 0.250 \\
Part 1 & 0.366 & 0.483 & 0.266 & \\
Part 2 & 0.328 & 0.148 & & \\
Part 3 & 0.156 & & \\
\hline
\end{tabular}

A further important factor in the stabilization of the hydrophilic regions is the conformation of the cation, which can be either linear, as found in the ordered region, or bent, as found in the disordered region, as defined by the values of the $N(1)-C(7)-C(8)-C(9)$ torsion angle listed in Table 8.5. In the bent conformer, the imidazolium ring is approximately orthogonal to the plane formed by the alkyl chain, as indicated by the value of the $\mathrm{C}(2)-\mathrm{N}(1)-\mathrm{C}(7)-\mathrm{C}(8)$ torsion angle. The $C(2)-N(1)-C(7)-C(8)$ and $N(1)-C(7)-C(8)-C(9)$ torsion angles define the tilt and the inclination of imidazolium ring, respectively, with respect to the plane formed by the alkyl chain. In the hydrophobic layers, the cation side chains form an interdigitated pattern, as found in form I. 
Table 8.5. Summary of dihedral angles of the decyl side chain of $\left[\mathrm{C}_{10} \mathrm{mim}\right]^{+}$cation and the reference angle $\mathrm{C}(2)-\mathrm{N}(1)-\mathrm{C}(7)-\mathrm{C}(8)$ seen in the low-temperature structures of form I and II-a. Highlighted values for Res2 of form II-a are responsible for the observed curving of the alkyl chain discussed in the main text.

\begin{tabular}{|c|c|c|c|c|c|}
\hline \multirow{2}{*}{ Dihedral angle $/^{\circ}$} & \multirow{2}{*}{ Form I (LT) } & \multicolumn{4}{|c|}{ Form II-a* } \\
\hline & & Res1 & Res2 & Res3 & Res3' \\
\hline $\mathrm{C}(2)-\mathrm{N}(1)-\mathrm{C}(7)-\mathrm{C}(8)$ & $125.51(0.17)$ & $134.32(0.32)$ & $131.84(0.34)$ & $71.46(0.88)$ & $71.56(1.41)$ \\
\hline$N(1)-C(7)-C(8)-C(9)$ & $-175.95(0.14)$ & $-169.59(0.27)$ & $-179.45(0.29)$ & $53.78(0.58)$ & $68.26(0.81)$ \\
\hline$C(7)-C(8)-C(9)-C(10)$ & $-179.12(0.15)$ & $-178.33(0.29)$ & $172.38(0.31)$ & \multicolumn{2}{|c|}{$176.22(0.35)$} \\
\hline$C(8)-C(9)-C(10)-C(11)$ & $-178.80(0.15)$ & $-179.10(0.30)$ & $-178.08(0.31)$ & \multicolumn{2}{|c|}{$175.71(0.36)$} \\
\hline $\mathrm{C}(9)-\mathrm{C}(10)-\mathrm{C}(11)-\mathrm{C}(12)$ & $179.67(0.15)$ & $179.46(0.31)$ & $174.83(0.32)$ & \multicolumn{2}{|c|}{$-179.83(0.34)$} \\
\hline$C(10)-C(11)-C(12)-C(13)$ & $179.68(0.15)$ & $179.90(0.31)$ & $-178.78(0.31)$ & \multicolumn{2}{|c|}{$179.15(0.34)$} \\
\hline$C(11)-C(12)-C(13)-C(14)$ & $179.70(0.15)$ & $179.60(0.32)$ & $177.52(0.32)$ & \multicolumn{2}{|c|}{$179.30(0.32)$} \\
\hline$C(12)-C(13)-C(14)-C(15)$ & $179.13(0.15)$ & $-178.62(0.33)$ & $-179.76(0.32)$ & \multicolumn{2}{|c|}{$179.45(0.33)$} \\
\hline$C(13)-C(14)-C(15)-C(16)$ & $179.76(0.17)$ & $179.06(0.37)$ & $179.73(0.36)$ & \multicolumn{2}{|c|}{$179.12(0.33)$} \\
\hline
\end{tabular}

*other form II structures follow the same conformations as those of form II-a

A distinctive feature of form II is the linear, curved and linear character of the alkyl side chains of Res1, Res2 and Res3 cations, respectively. The slight inclination has been previously ${ }^{2}$ ascribed to the imidazolium $\mathrm{H}$-atoms of Res2 acting as a bridge for $\left\{\left[\mathrm{H}_{2} \mathrm{O}\right] \mathrm{Cl}\right\}_{2^{2-}}$ dimers. The slight curving is more pronounced in $\left[\mathrm{C}_{10 \mathrm{mim}} \mathrm{Cl}\right.$ compared to the structures described previously. Furthermore, the plane defining the side chain of Res1 is almost orthogonal to both planes formed by Res2 and Res3 side chains (Figure 8.11). The "curving" 2 of the alkyl chain was calculated during the structural refinement with SHELXL through the input of the command MPLA. A least square plane was calculated using the carbon atoms' positions of the alkyl chain. Deviations of each atom position from the calculated least square plane are listed in the following charts. Figure 8.13 shows that for a given chain length the curving of Res2 alkyl chain is not affected by the crystallisation conditions; however, the curving is clearly dependent on the length of the alkyl chain, as is clearly displayed in Figure 8.14.

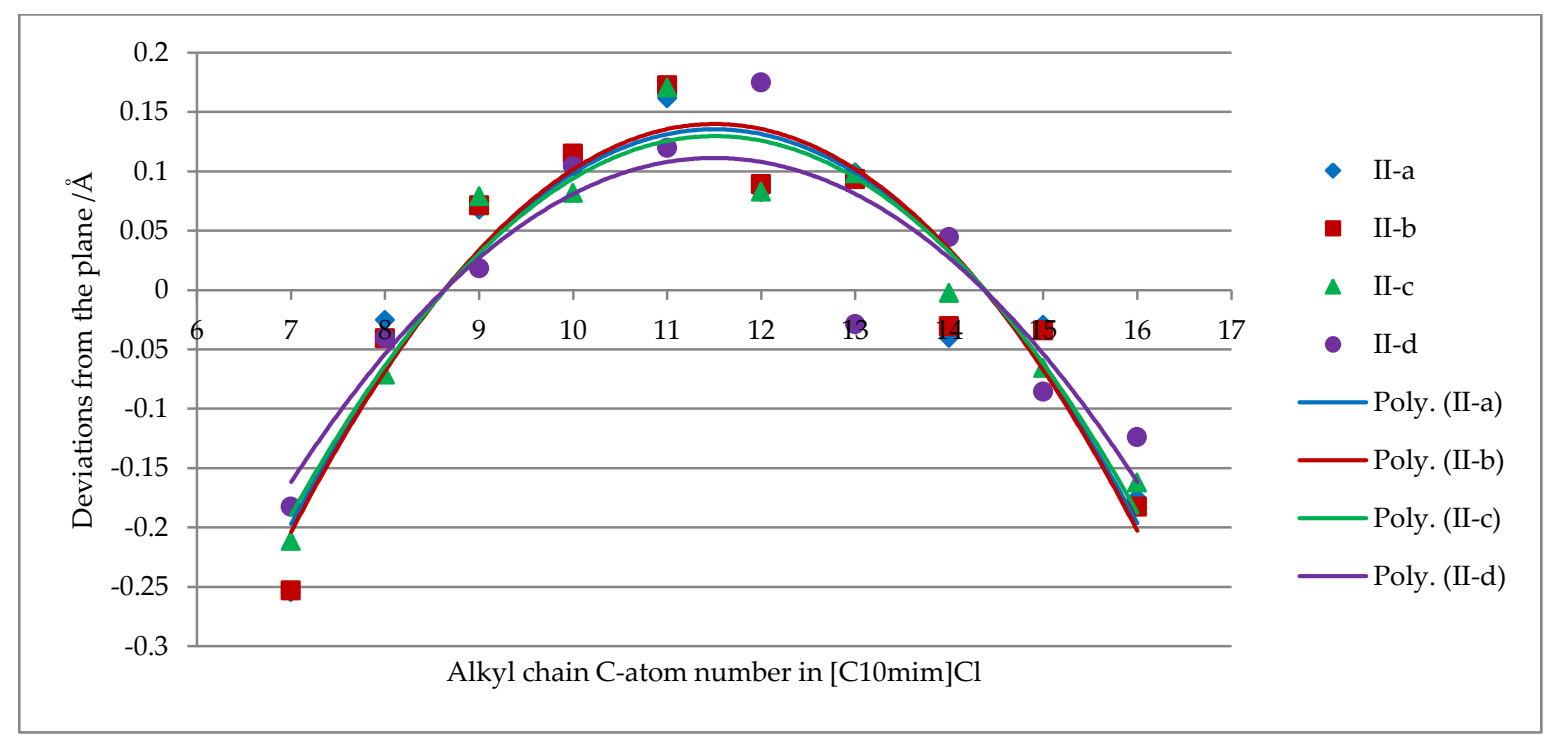

Figure 8.13. Comparison between the curving of the alkyl chains of Res2 in all form II structures reported in this work. A second order polynomial fit has been used to visually clarify the alkyl curving and should only be used as a guide to the eye. 


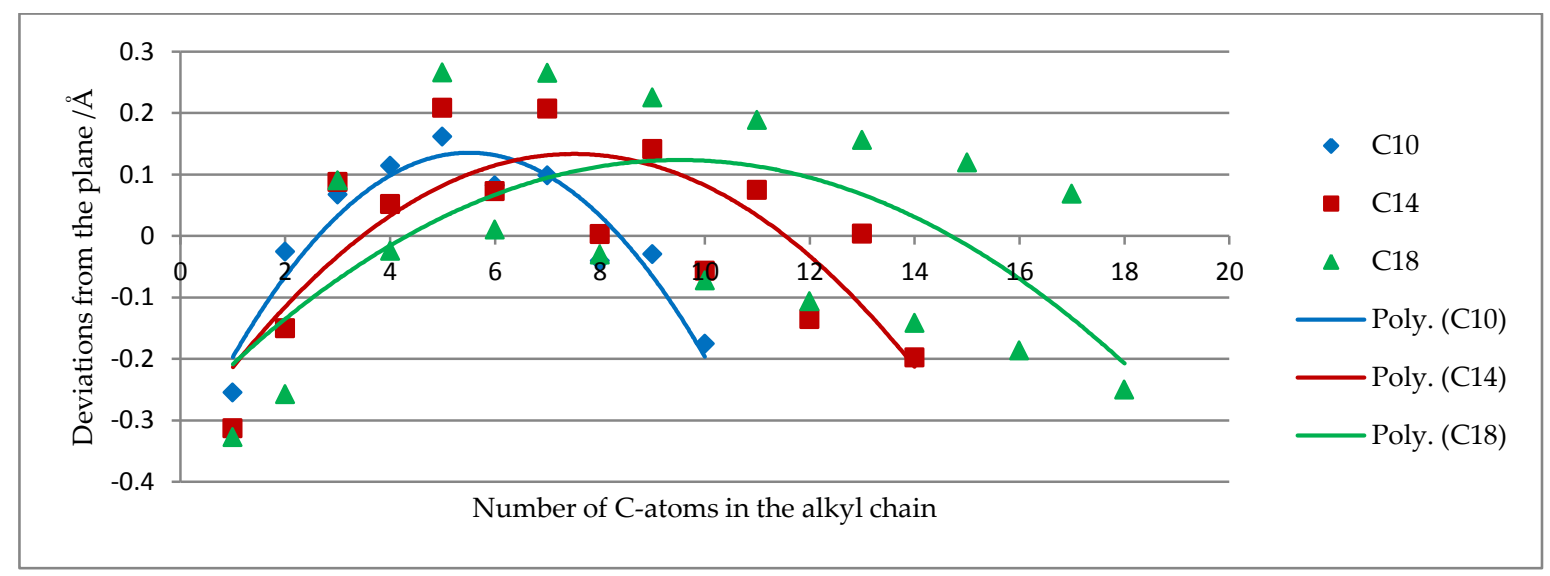

Figure 8.14. Comparison between the curving of the alkyl chains of Res2 in: form II-a, [C14mim]Cl and [C $18 \mathrm{mim}] \mathrm{Cl}$ structures. A second order polynomial fit (poly.) has been used to visually clarify the alkyl curving and should only be used as a guide to the eye.

A notable difference between the low-temperature and high-pressure structures, in addition to the differences in the site occupancies of the anions and water molecules, is that the imidazolium ring of Res3 exhibits tilting disorder at low temperature and ring flipping disorder at high-pressure conditions. Whereas at high pressure the disordered positions are equally occupied, this is not the case at low temperature (see Table 8.4). These structural differences can be directly ascribed to the different crystallisation conditions under which the structures were obtained. The observation of different conformations in the solid state indicate great structural variability in this form but should not be surprising given this form is obtained at low temperature by fast cooling rates and has a fast growth rate at high pressure, i.e. is obtained under kinetic control. Our experiments demonstrate that temperature annealing at high-pressure conditions is insufficient to induce complete ordering but results in the two cation positions being equally occupied.

\subsubsection{Crystal structures of form III}

[ $\left.\mathrm{C}_{10 \mathrm{mim}}\right] \mathrm{Cl}$ form III is a novel trihydrate form which, to the best of our knowledge, has never been reported for imidazolium-based ILs irrespective of alkyl chain length; rapid dissolution of the crystal on decompression prevented the investigation of recovery experiments. The structure of form III is fully ordered and is closely related to that of form I, with two further water molecules embedded in the hydrophilic region. As a consequence of this simple relationship, the main structural features describing form I are also valid for form III. The two new water molecules are inserted in the hydrophilic layer, increasing the distance between the imidazolium headgroups and creating an extended H-bonded network that can be described as a zigzag panel extending along the $a$-axis (Figure 8.15.5). 
When comparing the common fragments to both form I and form III structures, an important difference emerges, namely that the atomic positions of the anion and water molecule are interchanged. Whilst this does not alter the direction of the H-bonded zigzag chain, it enables the formation of a similar second chain bridged to the first via the additional water molecules (Figure 8.15.3). The repeating unit of this $3 \mathrm{D}$ H-bonded network is a $\left\{\left[\mathrm{H}_{2} \mathrm{O}\right]_{3} \mathrm{Cl}\right\}$ - ring. This motif is not uncommon and is found in 40 organic and inorganic structures in the CSD (V. 5.35 including updates to May 2014); however, the zigzag panel observed in form III is not seen in any of the reported structures. These findings provide further evidence that high pressure is able to combine interactions to form motifs that are new or uncommon at ambient pressure. ${ }^{40-44}$

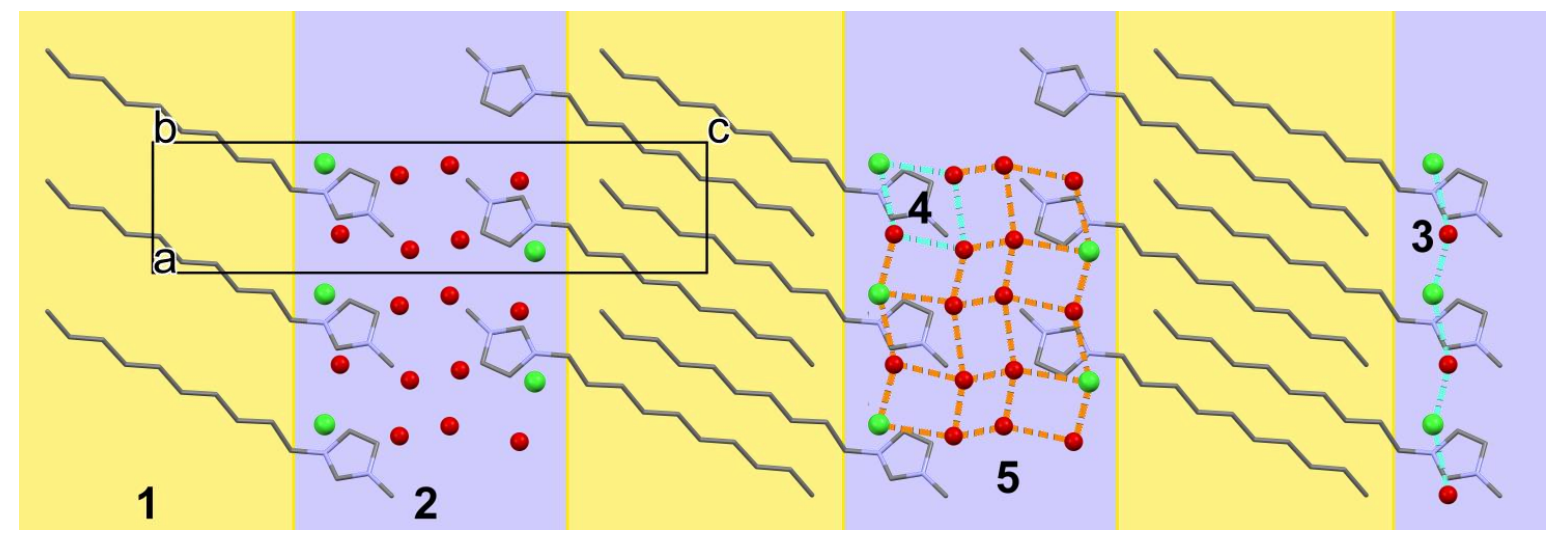

Figure 8.15. Crystal packing of [C $10 \mathrm{mim}] \mathrm{Cl}$ form III (0.6 GPa) seen along the $\boldsymbol{b}$-axis. H-atoms have been omitted for clarity. (1) Hydrophobic layer with interdigitated cation side chains; (2) Hydrophilic layer; (3) O$\mathrm{H} \cdots \mathrm{Cl} \mathrm{H}$-bonded chain along the $a$-axis; (4) $\{[\mathrm{H} 2 \mathrm{O}] 3 \mathrm{Cl}\}-\mathrm{H}$-bonded ring; (5) H-bonded zigzag panel.

\subsubsection{Liquid crystalline phases}

The title compound undergoes self-assembly and gelation upon water uptake; this property is not unknown for 1-alkyl-3-methylimidazolumhalides, ${ }^{2-4,8,15,19,45,46}$ and was described for the first time more than a decade ago by Firestone et al. for [C $10 \mathrm{~mm}] \mathrm{Br} .{ }^{19} \mathrm{~A}$ follow-up article in 2004 by the same group details the liquid crystalline changes that [ $\mathrm{C}_{10 \mathrm{mim}} \mathrm{Br}$ undergoes upon addition of water, using SAXS, infrared and NMR spectroscopy techniques. ${ }^{20}$ For this compound three lyotropic phases have been described having lamellar, hexagonal perforated layer with cubic character, and hexagonal structure for added water contents between $11 \%$ and $16 \%$ (w/w), of $17 \%$ $(\mathrm{w} / \mathrm{w})$ and of $40 \%(\mathrm{w} / \mathrm{w})$, respectively, with respect to a dried sample containing $1.5 \%(\mathrm{w} / \mathrm{w})$ water. Gelation was actually already observed for a composition containing $c a .5 \%(w / w)$ water.

In our work, we have observed very facile formation of water-rich lyotropic liquid crystalline phases of $\left[\mathrm{C}_{10 \mathrm{mim}}\right] \mathrm{Cl}$, which can be formed when the neat liquid uptakes air humidity or from an aqueous solution after a slow evaporation process (see Appendix 8.7 for details). We have observed that an increase in water content widens the temperature range of stability of these phases. This is in line with the thermal investigations of $\left[\mathrm{C}_{16} \mathrm{mim}\right] \mathrm{Cl} \cdot \mathrm{H}_{2} \mathrm{O}$ systems by Wu et al. ${ }^{46} \mathrm{At}$ 
least two, possibly three, lyotropic phases could be observed by polarised optical microscopy for [ $\left.\mathrm{C}_{10 \mathrm{mim}}\right] \mathrm{Cl}$ at ambient pressure and, additionally, as described in the experimental section, one at high pressure. The ambient-pressure results are qualitatively in good agreement with those on [C10mim]Br. We note that our dried sample has a higher starting water content, $c a .2 \%(w / w)$, than that of [ $\left.\mathrm{C}_{10 \mathrm{mim}}\right] \mathrm{Br} ;{ }^{20}$ we observed instantaneous formation of liquid crystals at $c a .4 \%$ added water. At $c a .19 \%(w / w)$ added water, the liquid crystalline phase looks distinctively different by optical microscopy and we presume this is a different phase. A further change was detected by microscopy at $c a .50 \%(\mathrm{w} / \mathrm{w})$. From $c a .57 .7 \%(\mathrm{w} / \mathrm{w})$ added water, dissolution of the liquid crystals is observed; at $c a .76 .7 \%(\mathrm{w} / \mathrm{w})$ added water, optical microscopy indicates a pure liquid state is obtained. We note that technically speaking a $c a .76 .7 \%(w / w)$ added water solution corresponds to a concentration of $c a .1 .1 \mathrm{M}$ solution and that a $1 \mathrm{M}$ solution was used to obtain the trihydrate crystalline phase at high pressure The phenomenon of liquid crystal formation at high-pressure conditions has not been extensively studied, with research focussed on polymeric compounds, one notable example being melting and crystallisation of polyethylene ${ }^{47}$. Interestingly, as described in the experimental section, upon further pressurization, the liquid crystal of [ $\left.\mathrm{C}_{10 \mathrm{mim}}\right] \mathrm{Cl}$ transforms into a crystalline phase. To the best of our knowledge, this is the first instance of such a pressuredriven phase transition. We note that for lipids, it has been observed that "The application of hydrostatic pressure to lyotropic phases in excess water tends to increase the lattice parameters of the phases. This effect is caused by the increase in conformational order of the hydrocarbon chains by pressure, and is the opposite effect to that of increasing temperature." 48

\subsection{5. $\left[\mathrm{C}_{10} \mathrm{mim}\right] \mathrm{Cl}$ as a crystallisation solvent for active pharmaceutical ingredients}

Having studied the behaviour of the neat IL and its aqueous solutions, our attention moved to trying to use this IL as a crystallisation solvent for active pharmaceutical ingredients (APIs). The aim of this study was to acquire some experience with using ILs as crystallisation media, particularly at high pressure. A recent example of a successful drug polymorphism study using water/IL mixtures as a co-solvent and the thermal shift method for crystallisation, is that of adefovir dipivoxil, an antiviral agent ${ }^{49}$ used in the treatment of the hepatitis B virus. For this compound a new polymorph and a new hemihydrate have been reported that could not be obtained when using conventional organic solvents..$^{50}$ Subsequently, the same authors reported a further new polymorph of the same compound obtained using mixtures of ionic liquids. ${ }^{51}$ The available literature reports experiments performed at ambient-pressure conditions only.

Preliminary experiments were performed using carbamazepine, 5-fluorouracil and paracetamol as APIs. The first two drugs were tested at ambient-pressure conditions, using dilute aqueous solutions, and the latter at high pressure using a $c a .1 \mathrm{M}$ aqueous solution of the IL; all 
materials were characterised by POM and single-crystal X-ray diffraction. For the ambient-pressure experiments the API was dissolved in the solution by applying heat ( $\left.T_{\max } 323 \mathrm{~K}\right)$; the mixture was cooled slowly to room temperature and crystallisation was observed upon slow evaporation. Carbamazepine dihydrate and the triclinic form for 5-fluorouracil were obtained in this manner. For the high-pressure experiments (Figure 8.16), crystals of paracetamol were dissolved in situ in the DAC upon heating to $343 \mathrm{~K}$. Crystallisation of [ $\left.\mathrm{C}_{10 \mathrm{mim}}\right] \mathrm{Cl}$ and paracetamol was observed at $c a$. 0.9 GPa. By cycling pressure and temperature, single crystals of paracetamol were grown and identified as the orthorhombic form of the drug, as confirmed by diffraction.

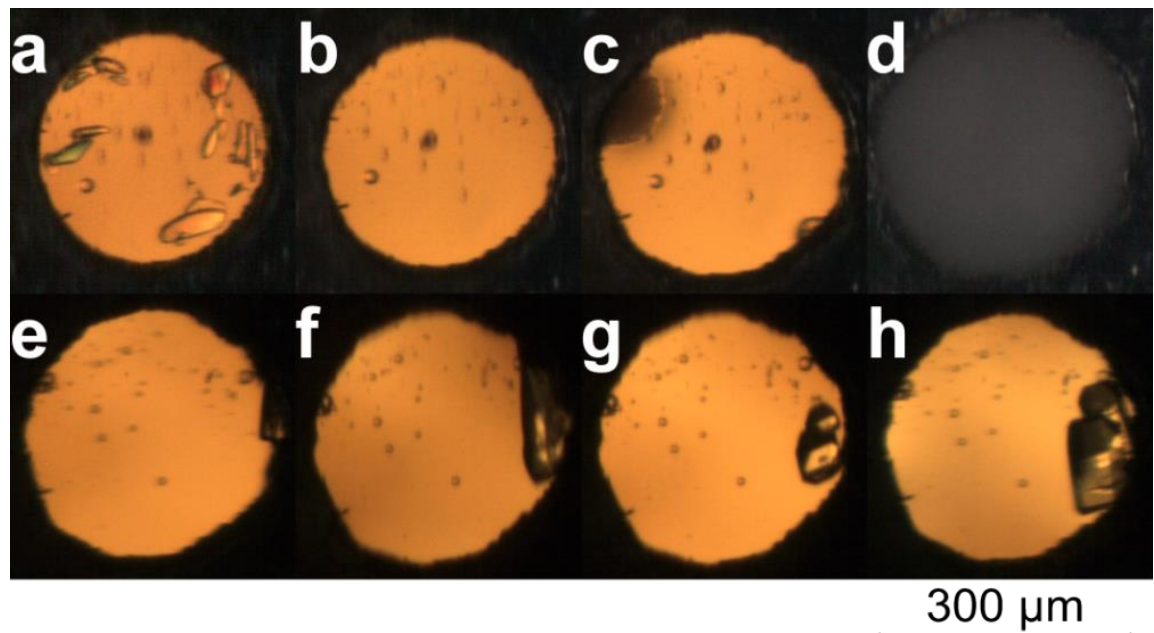

Figure 8.16. High-pressure crystallisation attempt of paracetamol in [C10mim]Cl solution in a DAC. a) DAC loaded at $0.16 \mathrm{GPa}$ with a crystalline powder of paracetamol in $c a .1 \mathrm{M}$ solution of $\left[\mathrm{C}_{10 \mathrm{mim}}\right] \mathrm{Cl}$, dissolution occurred after heating to $343 \mathrm{~K}$ and was complete in b); c) increasing pressure gradually from 0.30 to $0.95 \mathrm{GPa}$ through promotes the crystallisation of two populations of crystals; d) pressure dropped to $0.82 \mathrm{GPa}$ after complete crystallisation of the solution; e) releasing pressure to $0.29 \mathrm{GPa}$ dissolves the PTM a small crystalline cluster grown near the gasket is observed; f) increasing pressure to $0.44 \mathrm{GPa}$ promotes crystal growth; g) two

single crystals were obtained by cycling temperature up to $443 \mathrm{~K}$; h) cooling to ambient temperature promoted the growth of the crystals which was complete at the same pressure of $0.44 \mathrm{GPa}$.

In a second high-pressure experiment in which a different pressure and temperature range for crystallisation was explored, paracetamol monohydrate was crystallised instead (Figure 8.17).

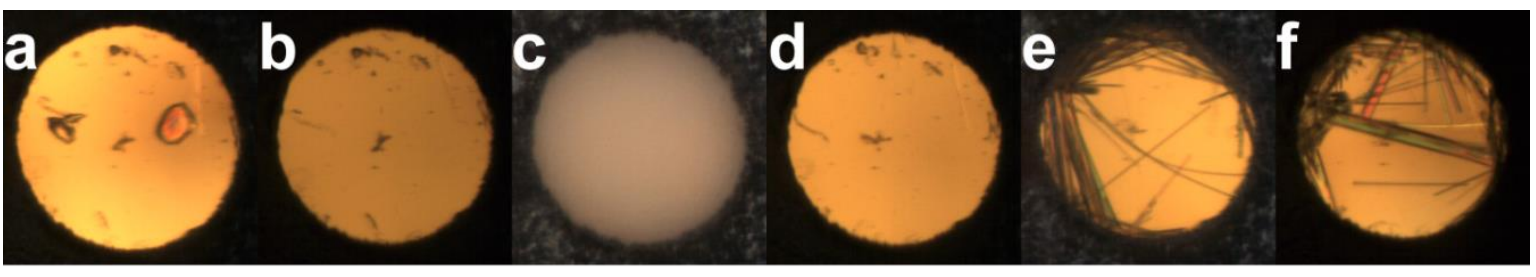

Figure 8.17. High-pressure crystallisation attempt of paracetamol in [C10mim]Cl solution in a DAC. a) DAC loaded at $0.10 \mathrm{GPa}$ with single crystals of paracetamol in $\mathrm{ca}$. $1 \mathrm{M}$ solution of [C1omim]Cl; b) complete dissolution at the same pressure after heating the DAC to $363 \mathrm{~K}$; c) crystalline powder of the PTM was obtained after increasing pressure to $0.70 \mathrm{GPa}$ and cooling the cell to 277 for 12 hours; d) the crystalline powder is dissolved after releasing the pressure to $0.56 \mathrm{GPa}$; e) crystallisation of needles after gentle heating to $313 \mathrm{~K}$ for 2 hours, pressure inside the DAC dropped to $0.47 \mathrm{GPa}$; f) cycling temperature up to $373 \mathrm{~K}$ and pressure promoted the growth of a large needle, final pressure inside the DAC was 0.66 GPa. 
Both paracetamol forms are easily obtained at ambient-pressure conditions and have also been reported to form at high pressure when using water, ethanol or methanol as solvents; ${ }^{22,53}$ at high pressure, concentrated solutions favour the crystallisation of paracetamol form II if pressure is increased gradually, while pressurisation of less concentrated solutions to pressure as high as 1 GPa favours the crystallisation of paracetamol monohydrate. ${ }^{53}$ In our experiments, the different forms are obtained as a function of the different pressure and temperature regimes used but not as a function of compression rate.

\subsubsection{General discussion}

[C10mim $] \mathrm{Cl}$ is a highly hygroscopic material that that readily forms gel-like mesophases at ambient pressure and temperature conditions. In the solid state, both form I, a true monohydrate, and form II, which crystallises with variable water content, from a pseudo-hemihydrate at high pressure to pseudo-monohydrate at low temperature, undergo a solid to gel phase transition when left in contact with air humidity. From the analysis of the hydrate structures obtained in this work, including the unprecedented availability of a trihydrate form, form III, uniquely obtained at high pressure, we speculate that mesophase formation might follow a mechanism that involves incorporation of water molecules in the hydrophilic region of the crystal, where the hydrophilic anions reside, and gradual disruption of the long-range crystalline order via cation disordering. On the basis of IR stretching frequencies, Firestone et al. commented on the $\mathrm{C}_{10}$ alkyl chain conformation in the fluid and the gel states of $\left[\mathrm{C}_{10 \mathrm{mim}}\right] \mathrm{Br}$, and reported trans/gauche disorder for both. ${ }^{20}$ This type of disorder was also confirmed by molecular dynamics simulations. ${ }^{54}$ Interestingly, single-crystal diffraction techniques show that in $\left[\mathrm{C}_{10 \mathrm{mim}}\right] \mathrm{Cl}$ hydrates the all-trans conformation exclusively exists in the three observed solid-state structures, with the exception of form II, where the gauche conformer is also observed in one of the three crystallographicallyindependent cations; the fact that this particular conformation is associated with cation disorder in the solid state and that form II exhibits the largest variation in water content suggest that form II hydrates are "trapped" structures, all close in energy, that give further insight into the configurations found in solution. Incidentally, we also note that form II is a Z' > 1 structure that appears to be stable with respect to transformation and hence probably qualifies as a "fossil relic of the fastest growing crystal nucleus". ${ }^{55}$

All low-temperature and high-pressure experiments conducted in this study are reproducible. An important parameter for preferential crystallisation of either form I or form II hydrates is water content: our experiments suggest that water content less than $c a .6 \%(\mathrm{w} / \mathrm{w})$ added water leads to form II crystals while contents higher than these values favour the formation of form I. The formation of hemihydrate and monohydrate structures from solutions in the range of $2-8 \%$ $(\mathrm{w} / \mathrm{w})$ water is not surprising. Technically speaking, 1:3 1:1 and 1:0.5 mixtures of [ $\left.\mathrm{C}_{10 \mathrm{mim}}\right] \mathrm{Cl}$ and 
water correspond to solutions containing ca. 17.3, 6.6 and $3.4 \%(\mathrm{w} / \mathrm{w})$ water. Downard et al. already noted that a related compound, $[\mathrm{C} 8 \mathrm{mim}] \mathrm{Cl}$, "absorbs approximately $6 \%(\mathrm{w} / \mathrm{w})$ at ambient conditions over a period of 2.5 hours, ${ }^{56}$ which roughly corresponds to a $1: 1$ mixture of [C8mim] $\mathrm{Cl}$ and water". In the case of [ $\left.\mathrm{C}_{10 \mathrm{mim}}\right] \mathrm{Cl}$ this concentration also yields and approximately equimolar stoichiometry. In addition to water content, kinetic factors seem to play an important role in [C $\mathrm{C}_{10 \mathrm{mim}} \mathrm{Cl}$ hydrate formation at low temperature, as exemplified by the influence of slow or fast cooling rates and sample history for obtaining either form I or form II crystals, respectively. This is in excellent agreement with the thermal polymorphism study on $\left[\mathrm{C}_{14} \mathrm{mim}\right] \mathrm{Cl}$ by Downard et al. ${ }^{2}$

Form I and II crystals at high pressure can be distinguished by optical microscopy: form I crystallises as well-defined plate-like crystals (Figure 8.3), whereas it is fairly easy to see intergrowth regions in form II crystals (Figure 8.4 and Figure 8.5); we speculate that the origin of these regions can be ascribed to the extensive disorder in the crystal; it is not unlikely that the disordered layer of form II acts as a nucleation point for new crystalline orientations, i.e. twinning. Given the variable water content of form II crystals, we investigated whether water uptake or release can also take place in the solid state. For this purpose we compressed a crystal of form II-d from $0.05 \mathrm{GPa}$ to $c$ a. $1 \mathrm{GPa}$. Single-crystal X-ray diffraction indicates that this modest pressure increase does not result in a structural change. High-pressure crystallisation of solutions containing ca. $60 \%(\mathrm{w} / \mathrm{w})$ water $(2.5 \mathrm{M})$ also yielded a crystallisation event; we suspected the presence of form I; however, due to poor solubility and the extremely slow growth rate of the powder material, it was not possible to confirm the identity of this form.

In light of the compound's hygroscopicity, we have found that the confined, very smallscale and sealed environment of the DAC is ideally suited for growing hydrates of the compound, offering better control over other techniques such as low-temperature crystallisation with a Linkam stage or in a capillary. In particular, the small sample volume of the DAC seems to be crucial for controlled crystallisation of the desired form: for instance, in the DAC it is easier to monitor and to ensure that the liquid-to-solid and the gel-to-solid phase transitions are complete. The coexistence of form I and II crystals and gel phases at low temperature can be explained by the much larger sample volumes used for these experiments. Interestingly, we have been unable to obtain a true monohydrate for form II, although such a hydrate probably does exit. We have also been unable to obtain structures with hydration number less than 0.45 ; as noted by Downard et al., "the nature of these layers, particularly the incorporation of water molecules via complex hydrogen-bonding interactions, may help to explain the persistent presence of water in imidazolium ionic liquids. It seems likely that the strong $\mathrm{O}-\mathrm{H} . . . \mathrm{Cl}$ hydrogen bonds in these compounds may be the reason that they are not easily dehydrated." ${ }^{2}$ Ultra high vacuum drying techniques might be useful towards this purpose..$^{57}$ 


\subsection{Conclusions}

The structural behaviour of the long chain imidazolium-based IL [ $\left.\mathrm{C}_{10} \mathrm{mim}\right] \mathrm{Cl}$-water mixtures has been investigated by low-temperature and high-pressure crystallisation methods and the crystalline phases fully characterized by single-crystal X-ray diffraction. On the basis of our experimental findings, we have been able to outline reliable crystallisation protocols for all forms; in particular we would like to highlight the use of the DAC as an ideal crystallisation vessel for hygroscopic compounds, providing both a controlled environment that can be entirely viewed with ease under an optical microscope and an effective tool for exploring and extending the crystallisation diagram. Form I, a monohydrate, crystallises from solutions containing $6-8 \%(\mathrm{w} / \mathrm{w})$ water and at low temperature its formation is favoured by slow cooling rates; its structure exhibits bilayers, in which the cations are alternating from layer to layer to create hydrophobic and hydrophilic regions. Form II, which incorporates a variable amount of water content (from pseudo hemihydrate to pseudo monohydrate) depending on sample treatment and environment, crystallises from solutions containing $<6 \%(\mathrm{w} / \mathrm{w})$ water; at low temperature its formation is favoured by fast cooling rates. The structure of form II exhibits a double bilayered structure characterized by ordered and disordered regions. This $Z^{\prime}>1$ structure can be considered a "fossil relic of the fastest growing crystal nucelus". ${ }^{55}$ Form III is a trihydrate that has been exclusively obtained under high-pressure conditions above $0.55 \mathrm{GPa}$ from solutions containing $>80 \%(\mathrm{w} / \mathrm{w})$ water. Its structure is closely related to that of form I. While homeotypic and isotypic structures of forms I and II have been reported previously for long chain imidazolium-based ILs, form III represents, to the best of our knowledge, the first example of a highly hydrated long chain imidazolium-based ionic liquid observed in the solid state. Form III has presently only been obtained at high pressure; however, we cannot rule out that this form cannot also be formed via a low-temperature crystallisation route using less diluted solutions. The formation of mesophases in the title compound has also been investigated by polarized optical microscopy and the results

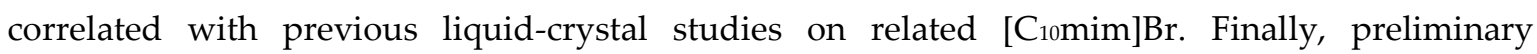
experiments demonstrate that $\left[\mathrm{C}_{10} \mathrm{mim}\right] \mathrm{Cl}$ water mixtures are suitable crystallisation media for crystallisation of drug molecules at both conditions of ambient and high pressure. Future studies will be performed to assess whether the combination of a long-chain amphiphilic IL together with water provides a widely applicable solvent matrix, e.g. for crystallisation by thermal, antisolvent, evaporation and high-pressure methods for both hydrophilic and hydrophobic drugs. 


\subsection{References}

(1) Chen, Y.; Song, W.; Xu, J.; Yang, X.-R.; Tian, D.-B. 1-Methyl-3-N-Tetra-Decyl-Imidazolium Bromide Monohydrate. Acta Crystallogr. 2009, E65, o2617.

(2) Downard, A.; Earle, M. J.; Hardacre, C.; Mcmath, S. E. J.; Nieuwenhuyzen, M.; Teat, S. J. Structural Studies of Crystalline 1-Alkyl-3-Methylimidazolium Chloride Salts. Chem. Mater. 2004, 16, 43-48.

(3) Getsis, A.; Mudring, A. V. 1-Dodecyl-3-Methylimidazolium Bromide Monohydrate. Acta Crystallogr. 2005, E61, o2945-o2946.

(4) Getsis, A.; Mudring, A. V. Imidazolium Based Ionic Liquid Crystals: Structure, Photophysical and Thermal Behaviour of [Cnmim] Br $\times \mathrm{xH} 2 \mathrm{O}(\mathrm{N}=12,14 ; \mathrm{X}=0,1)$. Cryst. Res. Technol. 2008, 43, 1187-1196.

(5) Guillet, E.; Imbert, D.; Scopelliti, R.; Bünzli, J. G. Tuning the Emission Color of EuropiumContaining Ionic Liquid-Crystalline Phases. Chem. Mater. 2004, 16, 4063-4070.

(6) Wei, Z.; Wei, X.; Fu, S.; Liu, J.; Zhang, D. 1-Hexadecyl-3-Methyl-Imidazolium Bromide Monohydrate. Acta Crystallogr. 2009, E65, o1159.

(7) Zhao, Y.-M.; Hu, X.-L.; Zhang, Q.-Y.; Guan, P.; Yu, J.-Y. [C16mim][Br]. Chinese J. Struct. Chem. 2009, 28, 1077.

(8) Zhao, Y.; Hu, X.; Zhang, Q.; Guan, P. Crystal Structure and Aggregation Behavior in Water of Ionic Liquid 1-Hexadecyl-3-Methylimidazolium Bromide. Mater. Lett. 2010, 64, 794-797.

(9) Allen, F. H. The Cambridge Structural Database: A Quarter of a Million Crystal Structures and Rising. Acta Crystallogr. Sect. B Struct. Sci. 2002, 58, 380-388.

(10) Swatloski, R. P.; Holbrey, J. D.; Rogers, R. D. Ionic Liquids Are Not Always Green: Hydrolysis of 1-Butyl-3-Methylimidazolium Hexafluorophosphate. Green Chem. 2003, 5 (4), 361-363.

(11) Dymek, C. J.; Grossie, D. A.; Fratini, A. V.; Adams, W. W. Evidence for the Presence of Hydrogen-Bonded Ion-Ion Interactions in the Molten Salt Precursor, 1-Methyl-3Ethylimidaazolium Chloride. J. Mol. Struct. 1989, 213, 25-34.

(12) Holbrey, J. D.; Reichert, W. M.; Nieuwenhuyzen, M.; Johnson, S.; Seddon, K. R.; Rogers, R. D. Crystal Polymorphism in 1-Butyl-3-Methylimidazolium Halides: Supporting Ionic Liquid Formation by Inhibition of crystallizationElectronic Supplementary Information (ESI) Available: Packing Diagrams for I and II; Table of Closest Contacts for I, I-Br and I. Chem. Commun. 2003, No. 14, 1636.

(13) Bowlas, C. J.; Bruceb, D. W.; Seddon, K. R.; Bruce, D. W.; Seddon, K. R.; Bruceb, D. W.; Seddon, K. R. Liquid-Crystalline Ionic Liquids. Chem. Commun. 1996, 1625-1626.

(14) Binnemans, K. Ionic Liquid Crystals. Chem. Rev. 2005, 105, 4148-4204.

(15) Pei, M.; Wu, Z.; Wang, L.; Wu, X.; Tao, X. Phase Behavior of Liquid Crystals Formed in [C $12 \mathrm{mim}] \mathrm{CI} / \mathrm{H} 2 \mathrm{O}$ and [C $12 \mathrm{mim}] \mathrm{CI} /$ Alcohols Systems. Chinese J. Chem. Phys. 2009, 22, 453459 .

(16) Yang, M.; Mallick, B.; Mudring, A.-V. V. On the Mesophase Formation of 1,3Dialkylimidazolium Ionic Liquids. Cryst. Growth Des. 2013, 13 (7), 3068-3077.

(17) Li, X.-W.; Zhang, J.; Dong, B.; Zheng, L.-Q.; Tung, C.-H. Characterization of Lyotropic Liquid Crystals Formed in the Mixtures of 1-Alkyl-3-Methylimidazolium Bromide/pXylene/water. Colloids Surfaces A Physicochem. Eng. Asp. 2009, 335, 80-87.

(18) Wu, J.; Zhang, J.; Zheng, L.; Zhao, X.; Li, N.; Dong, B. Characterization of Lyotropic Liquid Crystalline Phases Formed in Imidazolium Based Ionic Liquids. Colloids Surfaces A 
Physicochem. Eng. Asp. 2009, 336, 18-22.

(19) Firestone, M. A.; Dzielawa, J. A.; Zapol, P.; Curtiss, L. a.; Seifert, S.; Dietz, M. L. Lyotropic Liquid-Crystalline Gel Formation in a Room-Temperature Ionic Liquid. Langmuir 2002, 18 (20), 7258-7260.

(20) Firestone, M. A.; Rickert, P. G.; Seifert, S.; Dietz, M. L. Anion Effects on Ionogel Formation in N,N'-Dialkylimidazolium-Based Ionic Liquids. Inorganica Chim. Acta 2004, 357, 39913998.

(21) Bowers, J.; Butts, C. P.; Martin, P. J.; Vergara-Gutierrez, M. C.; Heenan, R. K. Aggregation Behavior of Aqueous Solutions of Ionic Liquids. Langmuir 2004, 20, 2191-2198.

(22) Goodchild, I.; Collier, L.; Millar, S. L.; Prokes, I.; Lord, J. C. D.; Butts, C. P.; Bowers, J.; Webster, J. R. P.; Heenan, R. K. Structural Studies of the Phase, Aggregation and Surface Behaviour of 1-Alkyl-3-Methylimidazolium Halide + Water Mixtures. J. Colloid Interface Sci. 2007, 307, 455-468.

(23) Saouane, S.; Norman, S. E.; Hardacre, C.; Fabbiani, F. P. A. Pinning down the Solid-State Polymorphism of the Ionic Liquid [bmim][PF6]. Chem. Sci. 2013, 4 (3), 1270-1280.

(24) Merrill, L.; Bassett, W. A. Miniature Diamond Anvil Pressure Cell for Single Crystal X-Ray Diffraction Studies. Rev. Sci. Instrum. 1974, 45, 290-294.

(25) Moggach, S. A.; Allan, D. R.; Parsons, S.; Warren, J. E. Incorporation of a New Design of Backing Seat and Anvil in a Merrill-Bassett Diamond Anvil Cell. J. Appl. Crystallogr. 2008, 41 (2), 249-251.

(26) Piermarini, G. J.; Block, S.; Barnett, J. D.; Forman, R. A. Calibration of the Pressure Dependence of the R1 Ruby Fluorescence Line to 195 Kbar. J. Appl. Phys. 1975, 46 (6), 27742780.

(27) Bruker-AXS. SAINT Version 8.34A, Madison, Wisconsin, USA. SAINT version 8.34A: Madison, Wisconsin, USA 2013.

(28) Dawson, A.; Allan, D. R.; Parsons, S.; Ruf, M. Use of a CCD Diffractometer in Crystal Structure Determinations at High Pressure. J. Appl. Crystallogr. 2004, 37 (3), 410-416.

(29) Sheldrick, G. M. SADABS Version 2008-1, Bruker-AXS, Madison, Wisconsin, USA. SADABS Version 2008-1, 2008, Bruker-AXS: Madison, Wisconsin, USA 2008.

(30) Parsons, S. SHADE, The University of Edinburgh: Scotland. The University of Edinburgh: Edinburgh, Scotland 2004.

(31) Sheldrick, G. M. A Short History of SHELX. Acta Crystallogr. Sect. A Found. Crystallogr. 2008, $64,112-122$.

(32) Hübschle, C. B.; Sheldrick, G. M.; Dittrich, B. ShelXle: A Qt Graphical User Interface for SHELXL. J. Appl. Crystallogr. 2011, 44, 1281-1284.

(33) Grade Web Server. Global Phasing Limited 2014.

(34) Sheldrick, G. M. Crystal Structure Refinement with SHELXL. Acta Crystallogr. Sect. C Struct. Chem. 2015, $71(\mathrm{Md}), 3-8$.

(35) Gelbrich, T.; Hursthouse, M. B. A Versatile Procedure for the Identification, Description and Quantification of Structural Similarity in Molecular Crystals. CrystEngComm 2005, 7, 324336.

(36) Spackman, M. A.; Byrom, P. G. A Novel Definition of a Molecule in a Crystal. Chem. Phys. Lett. 1997, 267, 215-220.

(37) Fabbiani, F. P. A.; Dittrich, B.; Florence, A. J.; Gelbrich, T.; Hursthouse, M. B.; Kuhs, W. F.; Shankland, N.; Sowa, H. H. Crystal Structures with a Challenge: High-Pressure 
Crystallisation of Ciprofloxacin Sodium Salts and Their Recovery to Ambient Pressure. CrystEngComm 2009, 11 (7), 1396-1406.

(38) Spackman, M. A.; Jayatilaka, D. Hirshfeld Surface Analysis. CrystEngComm 2009, 11, 19-32.

(39) Spackman, M. a.; McKinnon, J. J. Fingerprinting Intermolecular Interactions in Molecular Crystals. CrystEngComm 2002, 4, 378-392.

(40) Aniola, M.; Olejniczak, A.; Katrusiak, A. Pressure-Induced Solvate Crystallization of 1,4Diazabicyclo[2.2.2]octane Perchlorate with Methanol. Cryst. Growth Des. 2014, 14, 21872191.

(41) Boldyreva, E. V. Multicomponent Organic Crystals at High Pressure. Zeitschrift für Krist. Cryst. Mater. 2014, 229, 236-245.

(42) Fabbiani, F. P. A.; Allan, D. R.; Parsons, S.; Pulham, C. R. An Exploration of the Polymorphism of Piracetam Using High Pressure. CrystEngComm 2005, 7 (29), 179.

(43) Francesca P. A. Fabbiani; Allan, D. R.; Parsons, S.; Pulham, C. R. Exploration of the HighPressure Behaviour of Polycyclic Aromatic Hydrocarbons: Naphthalene, Phenanthrene and Pyrene. Acta Crystallogr. 2006, B62, 826-842.

(44) Oswald, I. D. H.; Allan, D. R.; Day, G. M.; Motherwell, W. D. S.; Parsons, S. Realizing Predicted Crystal Structures at Extreme Conditions: The Low-Temperature and HighPressure Crystal Structures of 2-Chlorophenol and 4-Fluorophenol. Cryst. Growth Des. 2005, 5, 1055-1071.

(45) Bradley, A. E.; Hardacre, C.; Holbrey, J. D.; Johnston, S.; Mcmath, S. E. J.; Nieuwenhuyzen, M. Small-Angle X-Ray Scattering Studies of Liquid Crystalline 1-Alkyl-3Methylimidazolium Salts. Chem. Mater. 2002, 14, 629-635.

(46) Wu, F.-G.; Wang, N.-N.; Zhang, Q.-G.; Sun, S.-F.; Yu, Z.-W. Crystallization from the Micellar Phase of Imidazolium-Based Cationic Surfactants. J. Colloid Interface Sci. 2012, 374, 197-205.

(47) Yasuniwa, M.; Nakafuku, C.; Takemura, T. Melting and Crystallization Process of Polyethylene under High Pressure. Polym. J. 1973, 4, 526-533.

(48) Seddon, J. M.; Squires, A. M.; Conn, C. E.; Ces, O.; Heron, A. J.; Mulet, X.; Shearman, G. C.; Templer, R. H. Pressure-Jump X-Ray Studies of Liquid Crystal Transitions in Lipids. Philos. Trans. R. Soc. A Math. Phys. Eng. Sci. 2006, 364, 2635-2655.

(49) Perrillo, R.; Schiff, E.; Yoshida3, E.; Statler, A.; Hirsch, K.; Wright, T.; Gutfreund, K.; Lamy, P.; Murray, A. Adefovir Dipivoxil for the Treatment of Lamivudine-Resistant Hepatitis B Mutants. Hepatology 2000, 32 (1), 129-134.

(50) An, J.-H.; Kim, J.-M.; Chang, S.-M.; Kim, W.-S. Application of Ionic Liquid to Polymorphic Design of Pharmaceutical Ingredients. Cryst. Growth Des. 2010, 10 (7), 3044-3050.

(51) An, J.-H.; Kim, W.-S. Antisolvent Crystallization Using Ionic Liquids As Solvent and Antisolvent for Polymorphic Design of Active Pharmaceutical Ingredient. Cryst. Growth Des. 2013, 13, 31-39.

(52) Fabbiani, F. P. A.; Allan, D. R.; David, W. I. F.; Moggach, S. A.; Parsons, S.; Pulham, C. R. High-Pressure Recrystallisation?a Route to New Polymorphs and Solvates. CrystEngComm 2004, 6 (82), 504.

(53) Oswald, I. D. H.; Chataigner, I.; Elphick, S.; Fabbiani, F. P. A.; Lennie, A. R.; Maddaluno, J.; Marshall, W. G.; Prior, T. J.; Pulham, C. R.; Smith, R. I. Putting Pressure on Elusive Polymorphs and Solvates. CrystEngComm 2009, 11 (2), 359.

(54) Bhargava, B. L.; Klein, M. L. Molecular Dynamics Studies of Cation Aggregation in the Room Temperature Ionic Liquid [C10mim] $\mathrm{Br}]$ in Aqueous Solution. J. Phys. Chem. A 2009, 
113, 1898-1904.

(55) Steed, J. W. W. Should Solid-State Molecular Packing Have to Obey the Rules of Crystallographic Symmetry? CrystEngComm 2003, 5, 169-179.

(56) Seddon, K. R.; Stark, A.; Torres, M.-J. Influence of Chloride, Water, and Organic Solvents on the Physical Properties of Ionic Liquids. Pure Appl. Chem. 2000, 72, 2275-2287.

(57) Smith, E. F.; Garcia, I. J. V.; Briggs, D.; Licence, P. Ionic Liquids in Vacuo; Solution-Phase XRay Photoelectron Spectroscopy. Chem. Commun. 2005, 5633-5635. 


\subsection{Appendix: Qualitative analysis of $\left[\mathrm{C}_{10 \mathrm{mim}}\right] \mathrm{Cl}$ liquid crystal formation}

$\left[\mathrm{C}_{10 \mathrm{mim}}\right] \mathrm{Cl}$ is a transparent yellow liquid. As [ $\left.\mathrm{C}_{10 \mathrm{mim}}\right] \mathrm{Cl}$ is hygroscopic, it was dried twice prior to use: for $7 \mathrm{~h}$ at $80^{\circ} \mathrm{C}$ and for $6 \mathrm{~h}$ at the same temperature using a $1.4 \times 10^{-1}$ and $2.1 \times 10^{-1} \mathrm{mbar}$ vacuum, respectively. 11 vials of $\left[\mathrm{C}_{10} \mathrm{mim}\right] \mathrm{Cl}+\mathrm{H}_{2} \mathrm{O}$ mixtures were prepared inside a nitrogen glovebox with an estimated relative humidity of 4.5 to $6 \%\left(20-22^{\circ} \mathrm{C}\right)$. The vials were sealed inside the glovebox. Gentle heating of the vials associated with stirring ensured a homogenous distribution of the water in the ionic liquid. The following pictures describe the results of the polarised optical microscopy $(\mathrm{POM})$ of $\left[\mathrm{C}_{10 \mathrm{mim}}\right] \mathrm{Cl} \cdot \mathrm{H}_{2} \mathrm{O}$ mixtures for each sample composition. The pictures were taken just after opening the sealed vials. There are three distinguishable phases: The neat liquid, mesophases (as a function of different water concentration) and $\left[\mathrm{C}_{10 \mathrm{mim}}\right] \mathrm{Cl} \cdot \mathrm{H}_{2} \mathrm{O}$ solutions

Neat compound, $c a .2 \%$
(w/w) $\mathrm{H}_{2} \mathrm{O}$,
$\begin{aligned} & \text { corresponding to an } \\ & \text { approximate }\end{aligned}$
[C10mim $] \mathrm{Cl}: \mathrm{H}_{2} \mathrm{O}$
stoichiometry of $1: 0.3$
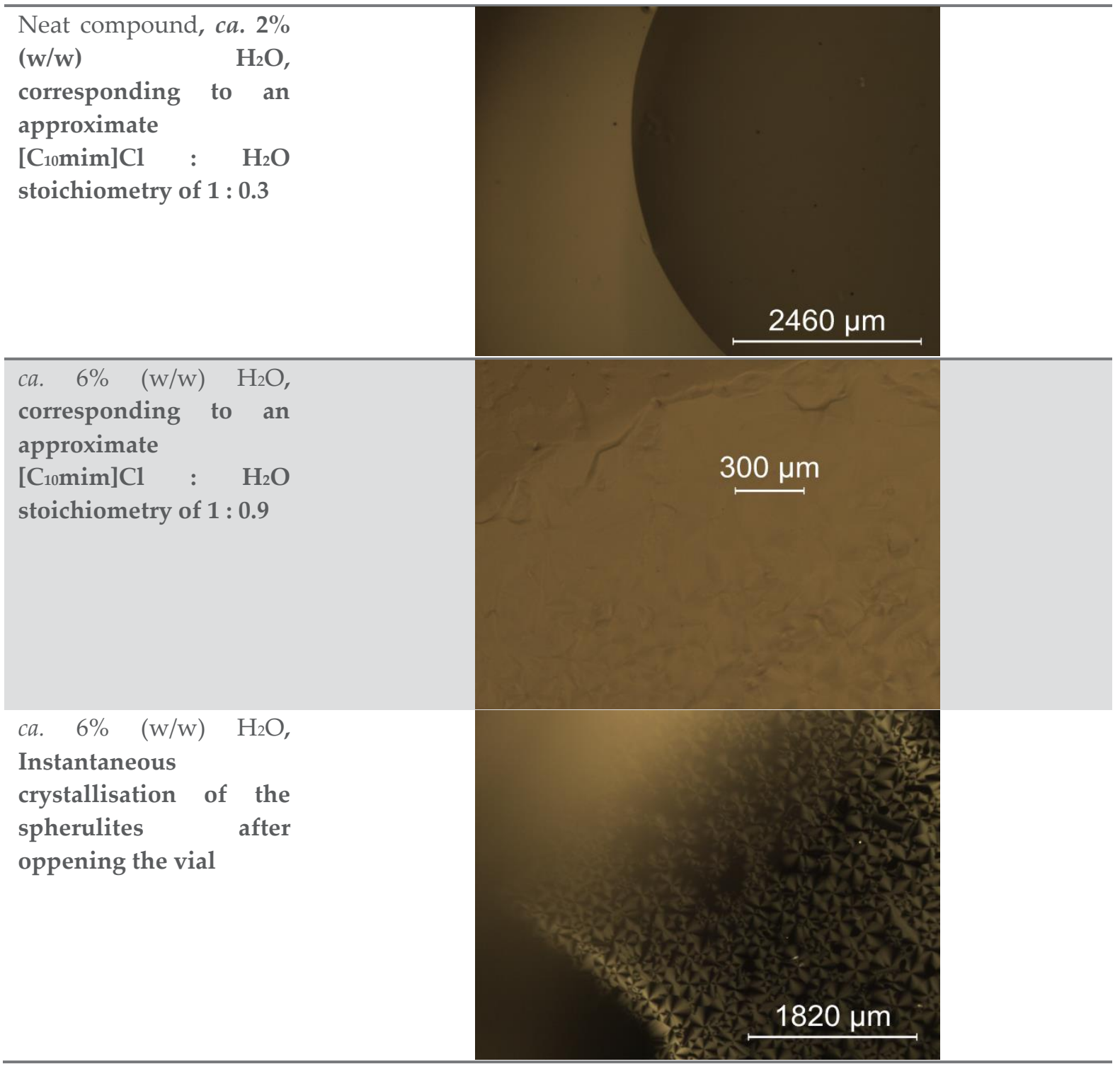


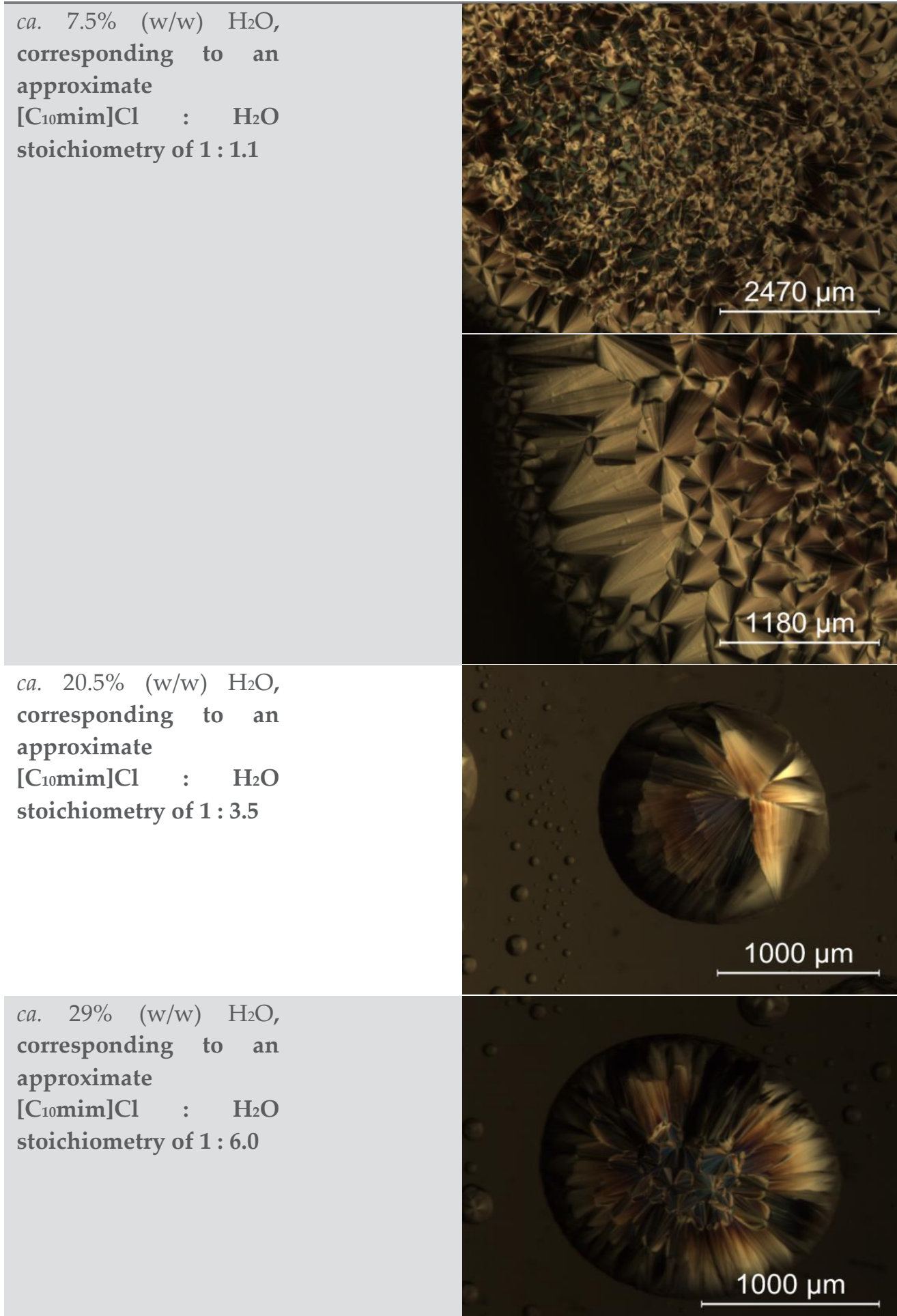




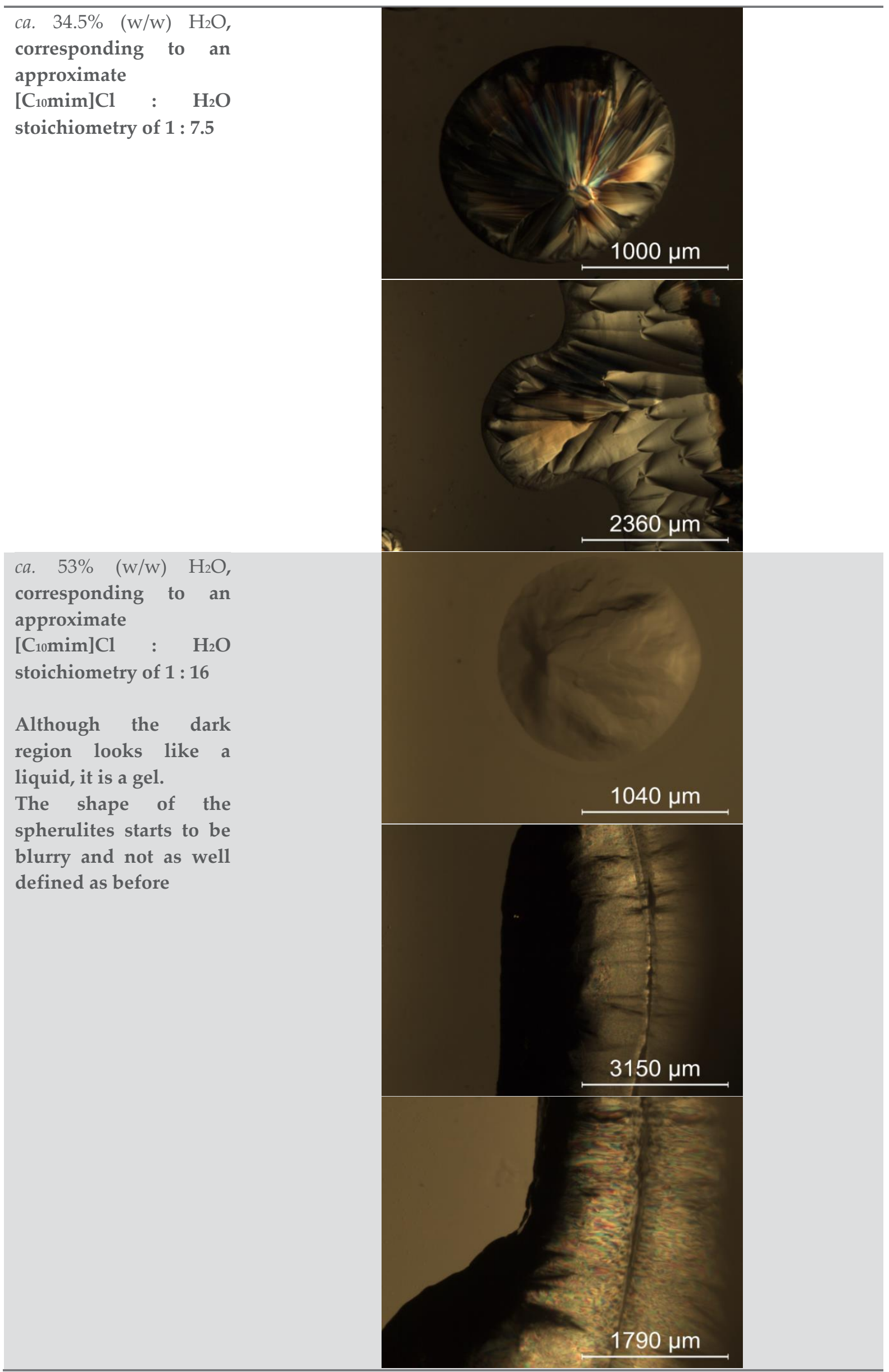




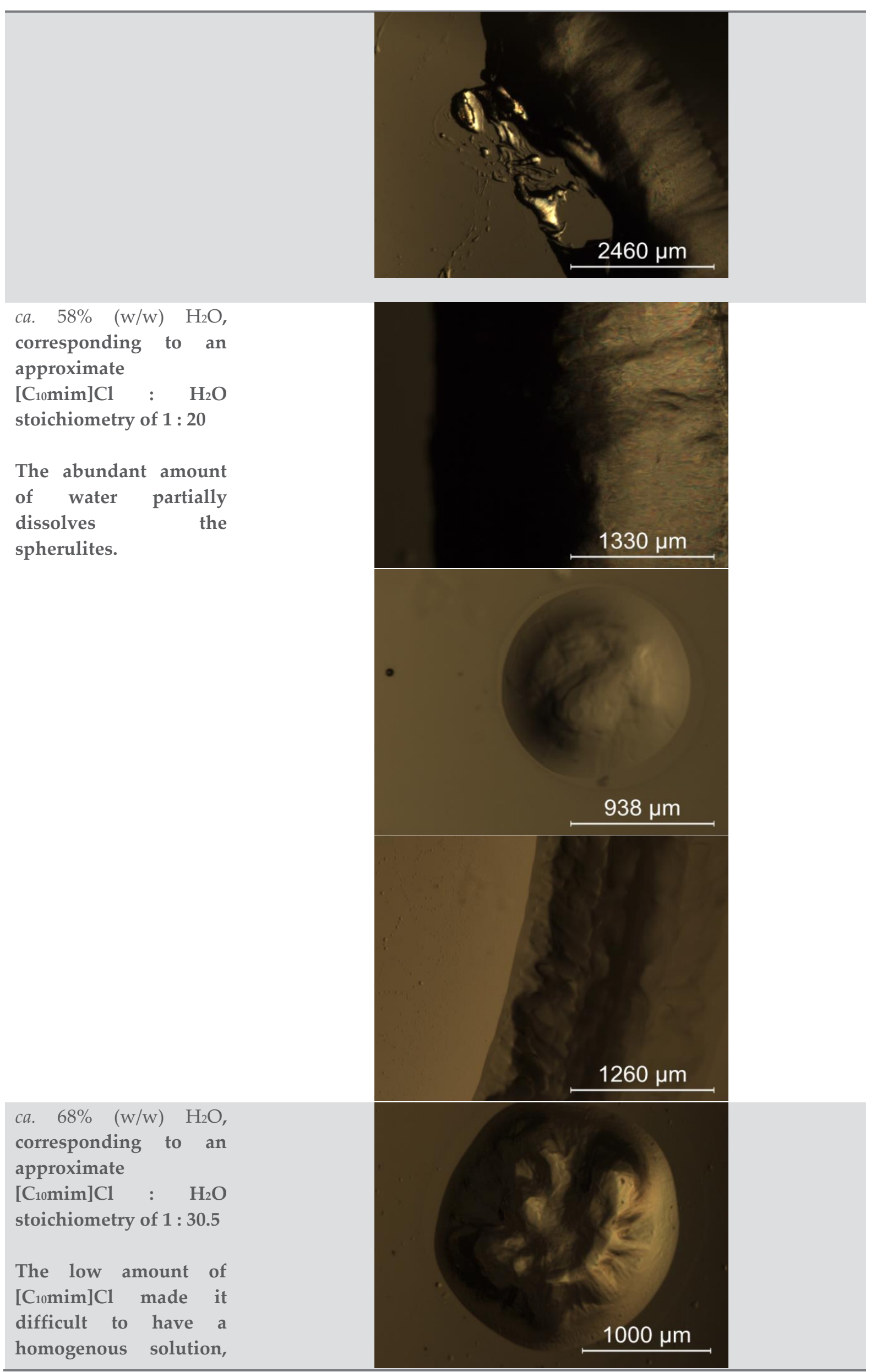




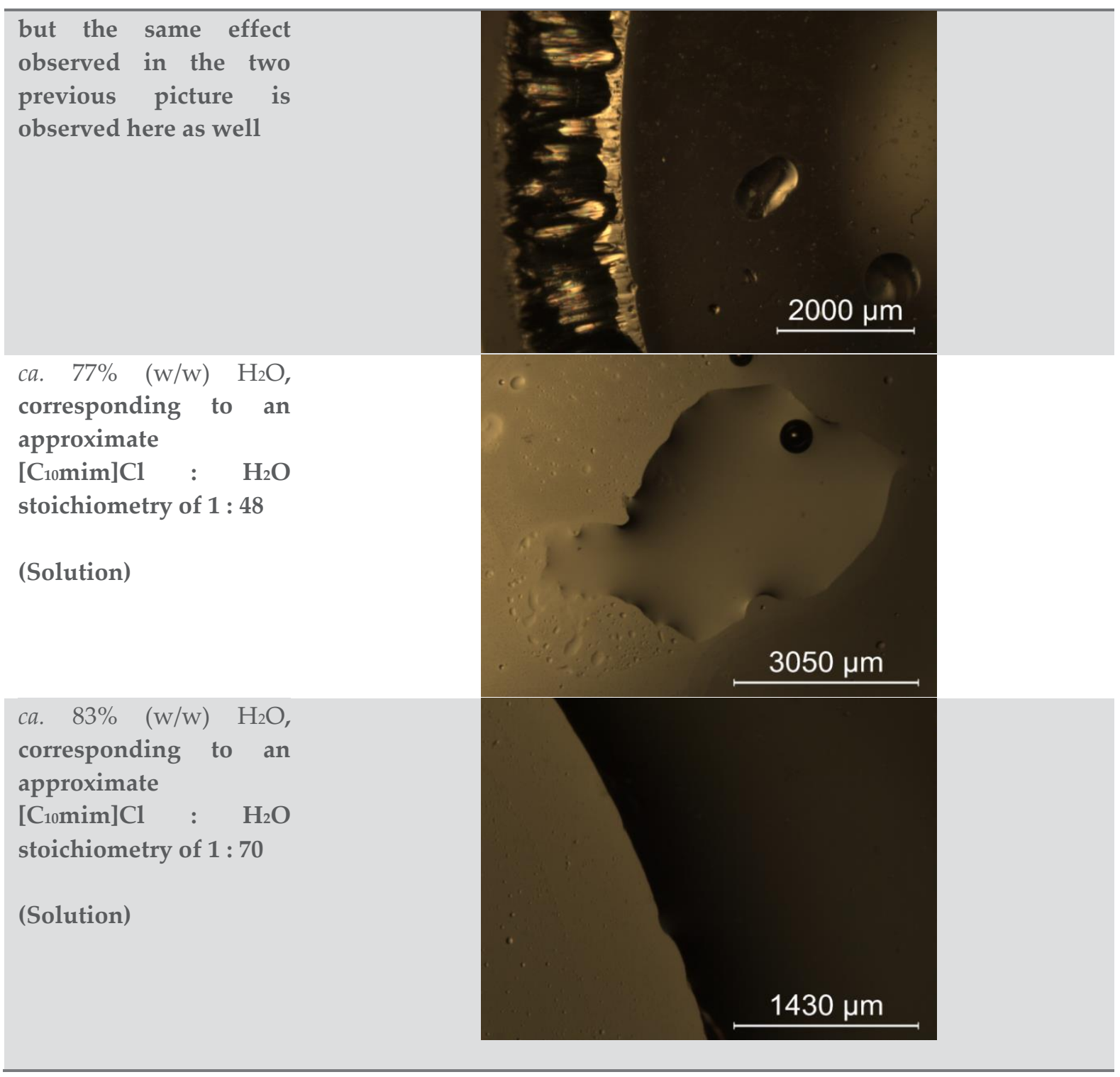

Figure 8.18. Qualitative analysis of [ $\left.\mathrm{C}_{10 \mathrm{mim}}\right] \mathrm{Cl}$ liquid crystal formation. 


\section{Chapter 9. Summary \& Outlook}

A series of experiments at high-pressure and low-temperature conditions on cyclodextrinbased inclusion complexes and on ionic liquids has been successfully carried out. This work shows that the application of high-pressure research using diamond-anvil cells can be further broadened to study complex systems such as cyclodextrin-based inclusion complexes and ionic liquids.

High-pressure techniques have been applied to investigate both the formation of cyclodextrin complexes in situ at high pressure, as well as the response of known complexes to pressure by means of compression studies. The main observation with all systems investigated is that water solubility of both $\alpha$ - and $\beta$-cyclodextrins drastically increases as a function of pressure. In the case of $\alpha$-cyclodextrin inclusion complexes, no crystals have been obtained by high-pressure recrystallisation; this observation is closely related to the use of water as pressure-transmitting medium and is probably related to the high water solubility of $\alpha$-cyclodextrin at ambient pressure. Further experiments should focus on probing further guest molecules and particularly on the use of non-aqueous solvents. $\beta$-cyclodextrin complexes can be crystallised at high pressure and form a novel superhydrated packing motif, as it has been shown for the $\beta$-cyclodextrin paracetamol system. Superhydration is accompanied by lower guest occupancy, $c a .133$, than observed at both ambient-pressure and low-temperature conditions, where 1:1 complexes are invariably obtained. In some experiments, crystallisation of the drug molecule alone, i.e. not as a complex, was observed, e.g. as demonstrated for the $\beta$-cyclodextrin·5-fluorouracil system. The high-pressure dissolution trend observed for $\beta$-cyclodextrin was used exploited to solubilise poorly-water soluble drugs, as illustrated for amoxicillin, showing that inclusion complex formation is very likely to occur in solution a high pressure. This mechanism is potentially of practical application and its applicability should be further investigated with other drug molecules. Use of spectroscopic techniques, such as NMR, Raman and UV-Vis spectroscopy, should also be undertaken to fully characterise inclusion complex formation in solution.

Diamond-anvil cells have revealed themselves as ideal crystallisation vessels for ionic liquids and have therefore played a key role in studying the solid-state behaviour of these compounds; in particular, a diamond-anvil cell offers a sealed environment, which can for instance be useful to control humidity, and the means for in situ microscopy, Raman spectroscopy and Xray diffraction. High-pressure and low-temperature studies have been performed on two of the most widely studied ionic liquids, namely 1-butyl-3-methylimidazolium hexafluorophosphate, [bmim] $\left[\mathrm{PF}_{6}\right]$, and 1-decyl-3-methylimidazolium chloride, $\left[\mathrm{C}_{10 \mathrm{mim}}\right] \mathrm{Cl}$. In the case of $[\mathrm{bmim}]\left[\mathrm{PF}_{6}\right]$, the existence of three polymorphs has been confirmed, with all polymorphs fully characterised by means of single-crystal X-ray diffraction. A detailed structural analysis, and most importantly, the 
availability or the first time of structural data, has demonstrated that a much wider range of cation conformers exists in the solid state than previously thought. Across the three phases and under different experimental conditions, a large variation in crystal packing densities and in structural disorder is observed. These variations are likely contributing driving forces for the observed polymorphic interconversions. $\left[\mathrm{C}_{10 \mathrm{mim}}\right] \mathrm{Cl}$ - water mixtures have also been the subject of an extended crystallisation study. By varying the amount of water in the $2-88 \%(w / w)$ range, eight distinct hydrates have been obtained, which belong to three distinct packing types. A trihydrate form has been obtained for a first time for an ionic liquid by using high-pressure crystallisation methods. Preliminary investigations of mesophase formation in $\left[\mathrm{C}_{10 \mathrm{mim}}\right] \mathrm{Cl}$ have revealed a potentially rich phase diagram and further studies, in particular at high pressure, should be performed to assess whether this phenomenon is widespread and whether it could be exploited for practical applications. Preliminary studies have shown that ILs may also be suitable crystallisation solvents for drug molecules for both ambient-pressure and high-pressure studies. Given the limited number of structural studies on crystalline phases of ILs available in the literature and the very large number of ILs available, future crystallisation studies of this class of compounds promise to be a very fertile research area. 